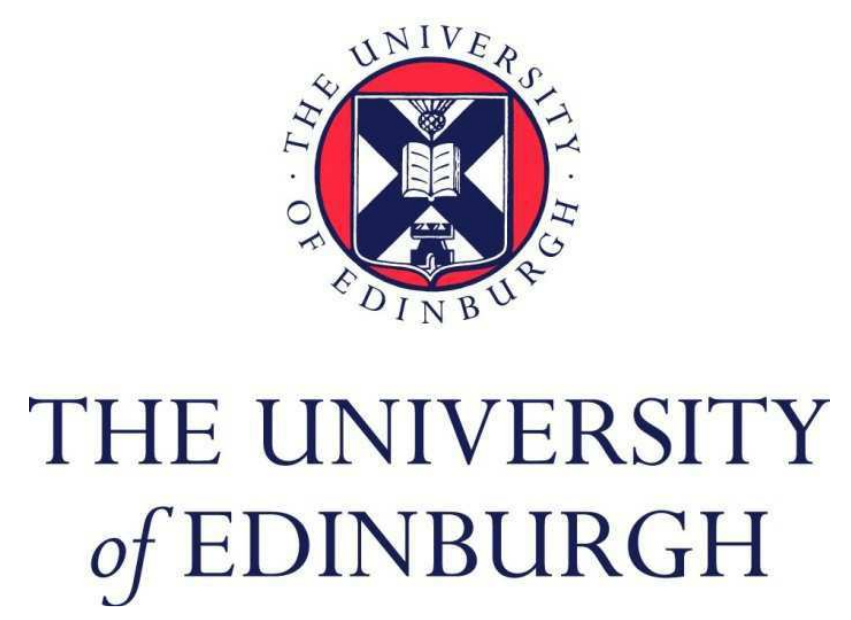

This thesis has been submitted in fulfilment of the requirements for a postgraduate degree (e.g. PhD, MPhil, DClinPsychol) at the University of Edinburgh. Please note the following terms and conditions of use:

This work is protected by copyright and other intellectual property rights, which are retained by the thesis author, unless otherwise stated.

A copy can be downloaded for personal non-commercial research or study, without prior permission or charge.

This thesis cannot be reproduced or quoted extensively from without first obtaining permission in writing from the author.

The content must not be changed in any way or sold commercially in any format or medium without the formal permission of the author.

When referring to this work, full bibliographic details including the author, title, awarding institution and date of the thesis must be given. 


\section{Study, Analysis and Application of Optical OFDM, Single Carrier (SC) and MIMO in Intensity Modulation Direct Detection (IM/DD)}

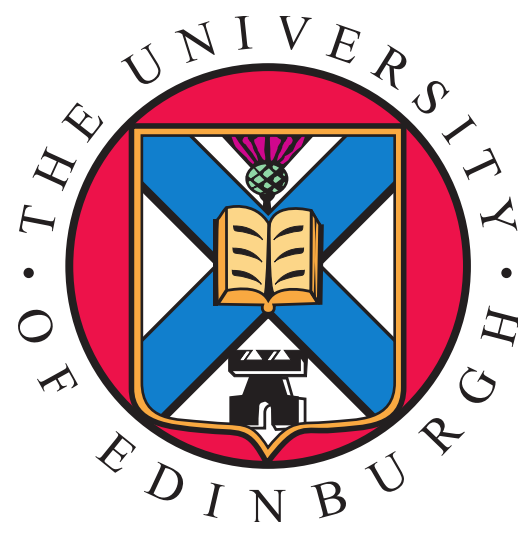

A thesis submitted for the degree of Doctor of Philosophy.

The University of Edinburgh.

January 2015 


\section{Abstract}

With the rapid growth of wireless data demands and saturation of radio frequency (RF) capacity, visible light communication (VLC) has become a promising candidate to complement conventional RF communication, especially for indoor short range applications. However the performance of the system depends on the propagation and type of system used. An optical Orthogonal Frequency Division Multiplexing (O-OFDM) together with multiple input multiple output (MIMO) in different scenario and modulation techniques are studied in the thesis.

A novel optical wireless communication (OWC) multi-cell system with narrow field of view (FOV) is studied. In this system the intensity modulated beam from four light sources are used for communication. The system allows beams to be concentrated in specific areas of the room to serve multiple mobile devices with low interference and hence increase system capacity. The performance of asymmetrically clipped optical orthogonal frequency division multiplexing (ACO-OFDM), direct current biased optical OFDM (DCO-OFDM) and single carrier (SC) modulation are then compared in this system considering single user and multiusers scenarios. The performance of the multi-cell is compared with single cell with wide FOV. It is shown that the capacity for multi-cell system increases with the number of users to 4 times the single user capacity. Also the findings show that multi-cell system with narrow beams can outperform a single wide beam system in terms of coverage area and hence average throughput of about 2.7 times the single wide beam system capacity.

One of the impairments in line of sight (LOS) OWC systems is coverage which degrades the performance. A mobile receiver with angular diversity detectors in MIMO channels is studied. The objective is to improve the rank of the channel matrix and hence system throughput. Repetition coding (RC), spatial multiplexing (SMP) and spatial modulation (SM) concepts are used to evaluate throughput across multiple locations in a small room scenario. A novel adaptive spatial modulation (ASM) which is capable of combating channel rank deficiency is devised. Since the receiver is mobile, the channel gains are low in some locations of the room due to the lack of LOS paths between transmitters and receivers. To combat the situation adaptive modulation and per antenna rate control (PARC) is employed to maximise spectral efficiency. The throughputs for fixed transmitters and receivers are compared with the oriented/inclined detectors for different cases. Angular diversity detectors offer a better throughput improvement 
than the state of the art vertical detectors, for example in ASM angular diversity receiver gives throughput of about 1.6 times that of vertical detectors. Also in SMP the angular detectors offer throughput about 1.4 times that of vertical detectors. SMP gives the best performance compared to RC, SM and ASM, for example SMP gives throughput about 2.5 times that of RC in both vertical detectors and angular diversity receivers. Again SMP gives throughput about 6 times that of SM in both vertical detectors and angular diversity receivers. Also SMP provides throughput about 2 times that of ASM in both vertical detectors and angular diversity receivers. ASM exhibit improvement in throughput about average factor of 3.5 times SM performance in both vertical detectors and angular diversity detectors.

As the performance of the system may be jeopardized by obstructions, specular and diffuse reflection models for indoor OWC systems using a mobile receiver with angular diversity detectors in MIMO channels are considered. The target is to improve the MIMO throughput compared to vertically oriented detectors by exploiting reflections from different reflecting surfaces in the room. The throughput across multiple locations in the small room by using RC, SMP and SM approaches is again evaluated. The results for LOS only channels against LOS with specular or diffuse reflection conditions, for both vertical and angular oriented receivers are then compared. The results show that exploiting specular and diffuse reflections provide significant improvements in link performance. For example the reflection coefficient $(\alpha)$ of 0.9 and the antenna separation of $0.6 \mathrm{~m}, \mathrm{RC}$ diffuse model shows throughput improvement of about 1.8 times that of LOS for both vertical detectors and angular diversity receivers. SM diffuse model shows throughput improvement of about 3 times that of LOS for both vertical detectors and angular diversity receivers. ASM diffuse model shows throughput improvement of about 2 times that of LOS for both vertical detectors and angular diversity receivers. SMP diffuse model shows throughput improvement of about 1.5 times that of LOS for both vertical detectors and angular diversity receiver. 


\section{Declaration of originality}

I hereby declare that the research recorded in this thesis and the thesis itself was composed and originated entirely by myself in the Institute for Digital Communications (IDCOM) of the School of Engineering at The University of Edinburgh.

Paul Fahamuel Mmbaga

January 2015 


\section{Acknowledgements}

I would like to express my deep gratitude to my supervisor, Prof. John Thompson, from the Institute for Digital Communications (IDCOM) at the University of Edinburgh, UK. His encouraging and unfailing comments lighted me the way for my research work and helped me stay on track over the years. His academic excellence, enthusiasm for research and quenchless desire to pursue novel ideas have deeply inspired me. His tireless advice and continuous support were essential for the successful completion of my PhD.

In addition, I would like to thank my second supervisor, Prof. Harald Haas, for his patience, guidance and support in my work. In particular, his constructive feedback and high attention to detail were invaluable for the successful completion of my PhD.

Many thanks to my colleagues in IDCOM for their support, encouragement and entertainment. Our fruitful discussions were of major importance for the continuous progress of my research.

This thesis is dedicated to my family and friends, the people who unconditionally stood by me. My deepest appreciation goes to their warming love, upbringing encouragement and motivation. A special word of appreciation goes to My wife Flora P Mmbaga for her untiring support and encouragement. You have been always there for me when I needed support. My inexplicable gratitude belongs to my mother, Namsifuel F Mmbaga, for her devotion and selfless care. Your being part of my life is invaluable to me, and words are insufficient to describe how thankful I am for this. I will be ungrateful if I don't express my sincere appreciations to My brother Joas F Mmbaga for his tireles moral and resources suport. May god bless you abundantly.

I finally thank god for all that he made possible for us. 


\section{Contents}

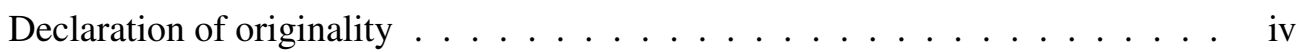

Acknowledgements ................... $\ldots$ v

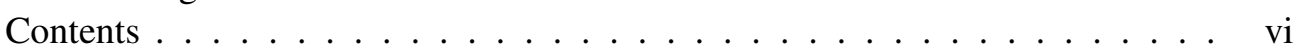

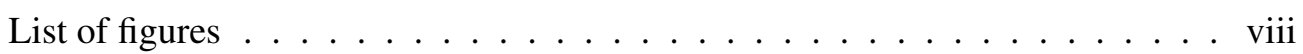

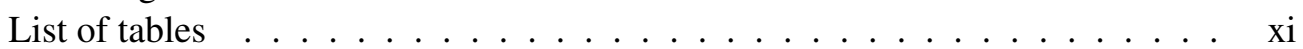

Acronyms and abbreviations $\ldots \ldots \ldots \ldots \ldots$ xii

Nomenclature . . . . . . . . . . . . . . . . . . . . . . . xv

1 Introduction 1

1.1 Motivation ....................... 1

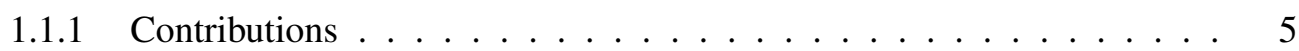

1.1 .2 Thesis Outline . . . . . . . . . . . . . . . . . . 7

1.1 .3 Chapter summary . . . . . . . . . . . . . . 8

2 Background 9

2.1 Evolution of wireless communications . . . . . . . . . . . . . . . 9

2.2 Optical wireless channel link . . . . . . . . . . . . . . . . . . . 13

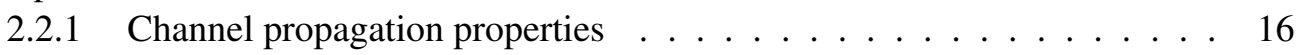

2.2 .2 Eye Safety . . . . . . . . . . . . . . . . . . . 16

2.2.3 Optical wireless channel model . . . . . . . . . . . . . . . 17

2.3 Single-carrier and multi-carrier modulation . . . . . . . . . . 20

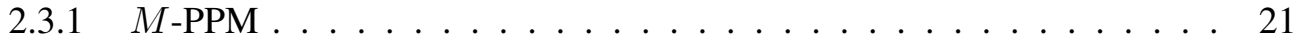

$2.3 .2 \quad M$-PAM ..................... 22

2.3.3 Optical OFDM using $M$-QAM . . . . . . . . . . . . . . . . . 24

2.4 Multiple Input Multiple Output (MIMO) . . . . . . . . . . . . . . . . . . 26

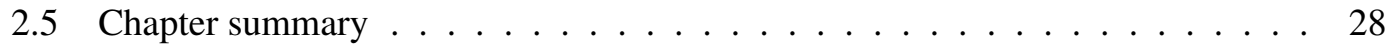

3 Application of Single Carrier (SC) and Optical OFDM, in Multi-Cell Multi-User $\begin{array}{ll}\text { VLC System } & \mathbf{3 0}\end{array}$

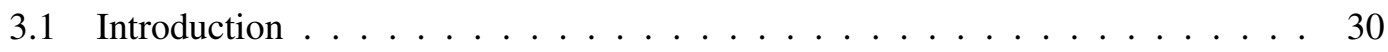

3.2 System descriptions . . . . . . . . . . . . . . . . . . . . 33

3.2.1 Basic optical wireless communication system . . . . . . . . . . . 33

3.2 .2 System model . . . . . . . . . . . . . . . . . . . . . . . . . 34

3.3 System under consideration . . . . . . . . . . . . . . . . . . . . 36

3.3.1 Asymmetrically clipped optical OFDM (ACO-OFDM) . . . . . . . . 36

3.3.2 Direct current biased optical OFDM (DCO-OFDM) . . . . . . . . . . . 38

3.3.3 Single Carrier-Pulse Amplitude Modulation (PAM) . . . . . . . . . . . 42

3.3.4 Simulation scenarios . . . . . . . . . . . . . . . . . . . 43

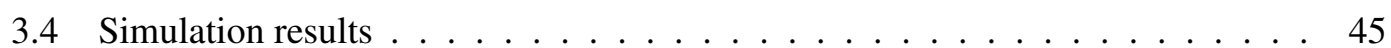

3.4.1 Single user single beam light simulation . . . . . . . . . . . . . . . 48

3.4.2 Single user multibeam light simulation . . . . . . . . . . . . . 48

3.4.3 Multiuser multibeam simulation . . . . . . . . . . . . . . . . 52

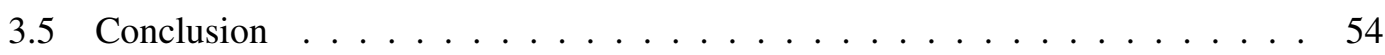


4 Improved indoor VLC MIMO channel capacity using receiver with angular diversity detectors $\quad \mathbf{5 5}$

4.1 Introduction . . . . . . . . . . . . . . . . . . . . 55

4.2 System model . . . . . . . . . . . . . . . . . . . 58

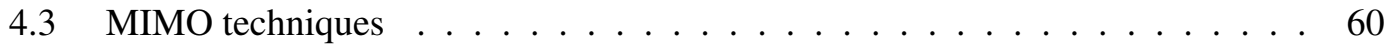

4.3.1 Repetition Coding $(\mathrm{RC}) \ldots \ldots \ldots 61$

4.3.2 Spatial Modulation (SM) . . . . . . . . . . . . . . . . . . . 63

4.3.3 Adaptive Spatial Modulation (ASM) . . . . . . . . . . . . . . . . . . . 64

4.3.4 Spatial Multiplexing (SMP) _. . . . . . . . . . . . . . . . . 66

4.4 System throughput evaluation . . . . . . . . . . . . . . . . . . . . 67

4.5 Simulation parameters and results . . . . . . . . . . . . . . . 68

4.5.1 Vertical detectors setup . . . . . . . . . . . . . . . . . . . . . . 69

4.5.2 Angular diversity detectors setup . . . . . . . . . . . . . . . . . 71

4.5.3 Comparison between Vertical detectors and Angular diversity detectors scenarios ..................... 71

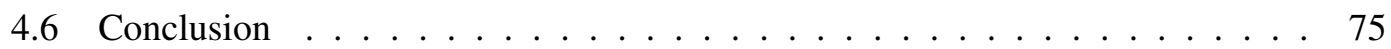

5 Performance analysis of indoor diffuse VLC MIMO channels using Angular Diversity Detectors $\quad 76$

5.1 Introduction . . . . . . . . . . . . . . . . . . . 76

5.2 System models . . . . . . . . . . . . . . . . . . . . . . . . . 79

5.3 Review of the MIMO techniques . . . . . . . . . . . . . . . . . 83

5.4 Simulation parameters and results $\ldots \ldots \ldots \ldots$. . . . . . . . . . . 84

5.4.1 Setting up Vertical detectors . . . . . . . . . . . . . . . 85

5.4.2 Comparison between Vertical detectors and Angular diversity detectors scenarios with specular and diffuse reflections. . . . . . . . . . . . 89

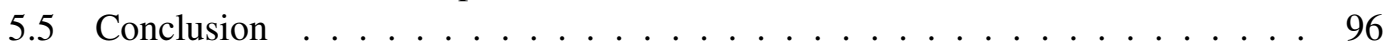

6 Conclusion $\quad 98$

6.1 Summary and major findings $\ldots \ldots \ldots$. . . . . . . . . . . . 98

6.2 Limitations and future work $\ldots \ldots \ldots 10$. . . . . . . . . . . . . . .

$\begin{array}{lr}\text { A Publications } & 103\end{array}$

$\begin{array}{ll}\text { References } & 145\end{array}$ 


\section{List of figures}

1.1 The UK Frequency Allocation $[2] \ldots \ldots \ldots$

1.2 Cisco Forecasts 15.9 Exabytes per Month of Mobile Data Traffic by 2018. Source: Cisco Visual Networking Index [3] . . . . . . . . . . . . . . . 3

1.3 The electromagnetic spectrum and the vast potential of unused, unregulated, safe green spectrum in the visible light part. The visible light spectrum is 10,000 times larger than the entire radio frequency spectrum. Source: SPIE Connecting minds advancing lights $[5] \ldots \ldots \ldots$. . . . . . . . . 4

2.1 The semaphore signalling alphanumeric system[30] . . . . . . . . . . . . . 10

2.2 The MIMO system setup. The signal transmitted from each transmit antenna is detected by all receiving antennas . . . . . . . . . . . . . . 12

2.3 Block diagram of a typical optical wireless system . . . . . . . . . . . . . 13

2.4 Classication of simple indoor OWC links according to the degree of directionality of the transmitter and receiver $[41] \ldots \ldots \ldots$

2.5 Geometries used in channel gains calculations (a) LOS link (b) Directed nonLOS link (c) Non-directed non-LOS link . . . . . . . . . . . . . . 18

2.6 Illustration of frequency spectra formats of (a) Single carrier and (b) Multi-carrier 20

2.7 Block diagram of single-carrier transmission in OWC using PAM and PPM . . 21

2.8 Illustration of $4-\mathrm{PPM} \ldots \ldots \ldots \ldots \ldots \ldots$

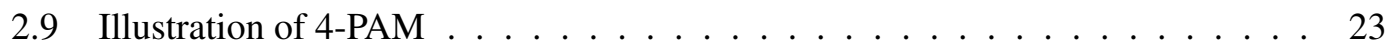

2.10 Block diagram of multi-carrier transmission using OFDM . . . . . . . . . . . 24

2.11 (a) D-BLAST and (b) V-BLAST coding for four antennas MIMO system . . . 27

3.1 Transmitter and receiver geometry in basic VLC system . . . . . . . . 33

3.2 (a) Block diagram of ACO-OFDM system (b) Data format of ACO-OFDM . . 37

3.3 Flow chart to illustrate throughput calculations . . . . . . . . . . . . 38

3.4 (a) Block diagram of DCO-OFDM system (b) Data format of DCO-OFDM . . 39

3.5 BER in AWGN versus $E_{b} / N_{0}$ for DCO-OFDM with DC bias of $7 \mathrm{~dB}$ and $13 \mathrm{~dB}$ using 4 QAM, 16 QAM, 64 QAM and 256 QAM (a) Simulated to compare with Figure 2 of [9] (b) Copy of Figure 2 of [9] . . . . . . . . . . . . . . . . 41

3.6 (a) Block diagram of SC system using PAM (b) Data format of SC system . . . 42

3.7 (a) Single beam light (b) Multibeam lights . . . . . . . . . . . . . . . . . . . 43

3.8 The output OFDM time signals for the ACO-OFDM and DCO-OFDM systems for $\mathrm{N}=32$. (a) Bipolar ACO-OFDM (not clipped) (b) Bipolar DCO-OFDM (before DC biasing) (c) Unipolar ACO-OFDM (clipped) (d) Unipolar DCOOFDM (after DC biasing) . . . . . . . . . . . . . . . . . . . . . . 46

3.9 BER in AWGN versus $E_{b} / N_{0}$ for bipolar 4 QAM and ACO-OFDM using 4 QAM, 16 QAM, 64 QAM, 256 QAM, and 1024 QAM (a) Simulated to compare with Figure 1 of [9] (b) Copy of Figure 1 of [9] . . . . . . . . . . . . . 47

3.10 One beam CDF plot Vs throughput . . . . . . . . . . . . . . . . . . . . . . . 49

3.11 One beam throughput coverage area . . . . . . . . . . . . . . . . . 49 
3.124 cells CDF plot Vs throughput (a) one detector receiver (b) Four detectors receiver ..................... 50

3.13 Four beam throughput coverage area . . . . . . . . . . . . 50

3.14 Four detectors receiver with random orientation . . . . . . . . . . . 51

3.15 ACO average throughput $\mathrm{Vs}$ number of users $\ldots \ldots \ldots \ldots$. . . . . . 52

3.16 DCO average throughput $\mathrm{Vs}$ number of users $\ldots \ldots \ldots \ldots$

3.17 PAM average throughput $V s$ number of users $\ldots \ldots \ldots . \ldots . \ldots 54$

4.1 Transmitter and receiver geometry used for channel gains calculations (a) General setup (b) Receiver with vertical axis detectors (c) Receiver with inclined axis detectors . . . . . . . . . . . . . . . . . . 58

4.2 Block diagram illustrating adaptive modulation . . . . . . . . . . . . 61

4.3 Illustration of RC operation with $N_{t}=4$ and $M=4$. Simultaneously emits the same signal from all transmitters . . . . . . . . . . . . . . 62

4.4 Illustration of SM operation with $N_{t}=4$ and $M=4$. The first two bits in the block of four bits determine the PAM symbol and second two bits determine the active LED [47] . . . . . . . . . . . . . . . . . . .

4.5 Illustration of SMP operation with $N_{t}=4$ and $M=4$. Independent data streams are simultaneously transmitted from all the transmitters . . . . . . . . . . 66

4.6 BER results (analytical and simulation) for MIMO vertical fixed detectors setup with TX separations of $0.2 \mathrm{~m}, 0.4 \mathrm{~m}$ and $0.6 \mathrm{~m}$ employing RC, SMP and SM (a) Simulated to compare with Figure 3 (a) of [47] (b) Copy of Figure 3 (a) of [47]

4.7 SMP CDF comparison between two elevation angles in angular diversity receiver detectors when LOS is considered (a) SMP, $10^{\circ}$ (b) SMP, $20^{\circ} \ldots \ldots$

4.8 Throughput Vs BER curves for SMP, SM and RC for angular diversity mobile receiver at $(2.05,1.95,0) \ldots \ldots \ldots \ldots \ldots$. . . . . . . . . . . . . . . . . .

4.9 The percentage distributions of MIMO channels matrix rank in 1000 locations of the room (a) Vertical detectors setup (b) Angular diversity detectors setup . . 73

4.10 CDF comparison between three MIMO techniques in vertically and angular diversity receiver detectors (a) RC (b) SMP (c) SM (d) ASM . . . . . . . . . .

5.1 Transmitter and receiver geometry used for channel gain calculations in specular reflection model . . . . . . . . . . . . . . . . . . . . . . . . 79

5.2 Transmitter and receiver geometry used for channel gain calculations (a) Diffuse reflection model (b) Diffuse reflection-wall geometry (c) Receiver with inclined and vertical axis detectors . . . . . . . . . . . . . . . . .

5.3 Percentage distributions of MIMO channels matrix rank in 1000 locations of the room (a) Vertical detectors setup (b) Angular diversity detectors setup (c \& e) Vertical detector setup with specular reflections (d \&f) Angular diversity detectors setup with specular reflections $(\mathrm{g} \& \mathrm{~h}$ ) Both vertical and angular detectors setups with diffuse reflections . . . . . . . . . . . . .

5.4 SMP CDF comparison between three elevation angles in angular diversity receiver detectors when $L O S, L O S+D f$ and $L O S+S p$ are considered, $\alpha=$ 0.5 (a) SMP, $10^{\circ}$ (b) SMP, $15^{\circ}$ (c) SMP, $20^{\circ} \ldots \ldots \ldots \ldots$ 
5.5 RC CDF comparison between three reflection coefficients in vertically and angular diversity receiver detectors when $L O S, L O S+S p$ and $L O S+D f$ are considered (a) $L O S$ Vs $L O S+S p, \alpha=0.3$ (b) $L O S+S p$ Vs $L O S+D f$,

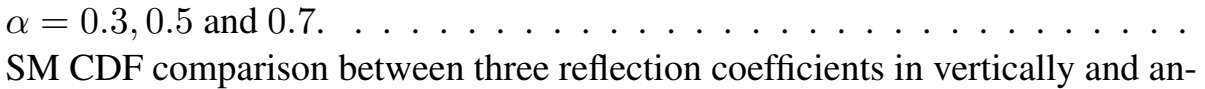

5.6 SM CDF comparison between three reflection coefficients in vertically and an-
gular diversity receiver detectors when $L O S, L O S+S p$ and $L O S+D f$ are considered (a) $L O S$ Vs $L O S+S p, \alpha=0.3$ (b) $L O S+S p$ Vs $L O S+D f$, $\alpha=0.3,0.5$ and $0.7 \ldots \ldots \ldots \ldots \ldots \ldots \ldots \ldots \ldots \ldots \ldots \ldots$

5.7 ASM CDF comparison between three reflection coefficients in vertically and
angular diversity receiver detectors when $L O S, L O S+S p$ and $L O S+D f$ are considered (a) $L O S$ Vs $L O S+S p, \alpha=0.3$ (b) $L O S+S p$ Vs $L O S+D f$, $\alpha=0.3,0.5$ and $0.7 \ldots \ldots \ldots \ldots \ldots \ldots$. . . . . . . . . . . 94

5.8 SMP CDF comparison between three reflection coefficients in vertically and angular diversity receiver detectors when $L O S, L O S+S p$ and $L O S+D f$ are considered (a) $L O S$ Vs $L O S+S p, \alpha=0.3$ (b) $L O S+S p$ Vs $L O S+D f$ comparison, $\alpha=0.3,0.5$ and 0.7 . 


\section{List of tables}

3.1 Parameters used for simulation . . . . . . . . . . . . . . . . 45

4.1 Parameters used for simulation . . . . . . . . . . . . . . . . . . . . 68

4.2 Simulation results for LOS with different antenna separations . . . . . . . . . . 72

5.1 Parameters used for simulation . . . . . . . . . . . . . . . 85

5.2 Simulation results for LOS with different antenna separations [from Table 4.2] . 86

5.3 Simulation results for LOS with Specular reflections _ . . . . . . . . . . 86

5.4 Simulation results for LOS with diffuse reflections $\ldots \ldots$. . . . . . . . . 86 


\section{Acronyms and abbreviations}

$3 G$

3GPP

$4 \mathrm{G}$

ACO-OFDM

$\mathrm{A} / \mathrm{D}$

AMC

ASM

ASE

AWGN

BLAST

BER

BPSK

BS

CAGR

CDMA

$\mathrm{CDF}$

CENELEC

$\mathrm{CP}$

CLT

CSI

$\mathrm{D} / \mathrm{A}$

DC

DCO-OFDM

DSP

DR

EU

FDMA

FFT

FOV
Third-Generation

Third-Generation Partnership Project

Fourth-Generation

Asymmetrically Clipped Optical Orthogonal Frequency Division Multiplexing Analog-to-Digital

Adaptive Modulation and Coding

Adaptive Spatial Modulation

Area Spectral Efficiency

Additive White Gaussian Noise

Bell Laboratories Layered Space-Time

Bit-Error Ratio

Binary Phase Shift Keying

Base Stations

Compound Annual Growth Rate

Code Division Multiple Access

Cumulative Distribution Function

European Committee for Electrotechnical Standardization

Cyclic Prefix

Central Limit Theorem

Channel State Information

Digital-to-Analog

Direct Current

Direct Current Biased Optical Orthogonal Frequency Division Multiplexing

Digital Signal Processor

Diffuse Reflection

European Union

Frequency Division Multiple Access

Fast Fourier Transform

Field Of View 


$\begin{array}{ll}\text { GPRS } & \text { General Packet Radio Service } \\ \text { GSM } & \text { Global System for Mobile communications } \\ \text { HPA } & \text { High-Power Amplier } \\ \text { ICI } & \text { Inter-Carrier Interference } \\ \text { IEEE } & \text { Institute of Electrical and Electronics Engineers } \\ \text { IFFT } & \text { Inverse Fast Fourier Transform } \\ \text { IM/DD } & \text { Intensity Modulation and Direct Detection } \\ \text { IR } & \text { Infrared } \\ \text { ISI } & \text { Inter-Symbol Interference } \\ \text { LED } & \text { Light Emitting Diode } \\ \text { LOS } & \text { Line Of Sight } \\ \text { LTE-A } & \text { Long-Term Evolution-Advanced } \\ \text { MIMO } & \text { Multiple-Input-Multiple-Output } \\ \text { ML } & \text { Maximum Likelihood } \\ \text { MMSE } & \text { Minimum Mean Squared Error } \\ \text { M-PAM } & \text { Multi-level Pulse Amplitude Modulation } \\ \text { M-PPM } & \text { Multi-level Pulse Position Modulation } \\ \text { M-QAM } & \text { Multi-level Quadrature Amplitude Modulation } \\ \text { NI } & \text { National Instruments } \\ \text { NIR } & \text { Near Infra-red } \\ \text { NLOS } & \text { Non-Line Of Sight } \\ \text { O/E } & \text { Optical-to-Electrical } \\ \text { O-OFDM } & \text { Optical Orthogonal Frequency Division Multiplexing } \\ \text { OOK } & \text { On-Off Keying } \\ \text { OWC } & \text { Optical Wireless Communication } \\ \text { P/S } & \text { Parallel-to-Serial } \\ \text { PAPR } & \text { Peak-to-Average-Power Ratio } \\ \text { PARC } & \text { Per Antenna Rate Control } \\ \text { PD } & \text { Photodiode } \\ \text { RC } & \text { Repetition Coding } \\ \text { RF } & \text { Radio Frequency } \\ \text { Receiver } \\ \text { Root Mean Square } \\ \text { Single Carrier } \\ \text { RA }\end{array}$




$\begin{array}{ll}\text { SER } & \text { Symbol-Error Rate } \\ \text { SISO } & \text { Single-Input-Single-Output } \\ \text { SNIR } & \text { Signal-to-Noise-and-Interference Ratio } \\ \text { SM } & \text { Spatial Modulation } \\ \text { SMP } & \text { Spatial Multiplexing } \\ \text { SNR } & \text { Signal-to-Noise Ratio } \\ \text { SR } & \text { Specular Reflection } \\ \text { S/P } & \text { Serial-to-Parallel } \\ \text { TDMA } & \text { Time Division Multiple Access } \\ \text { TIA } & \text { Transimpedance Amplifier } \\ \text { TX } & \text { Transmitter } \\ \text { U-OFDM } & \text { Unipolar Orthogonal Frequency Division Multiplexing } \\ \text { UV } & \text { Ultraviolet } \\ \text { VLC } & \text { Visible Light Communication } \\ \text { WiMAX } & \text { IEEE 802.16 wireless access standard } \\ \text { WLAN } & \text { Wireless Local Area network } \\ \text { ZF } & \text { Zero Forcing }\end{array}$




\section{Nomenclature}

* Linear convolution operator

- $\quad$ Vector dot product symbol

$(\cdot)^{*} \quad$ Complex conjugate

A Collection area of optical the detector

$B \quad$ Signal bandwidth

$B_{D C} \quad$ DC bias current

c Subcarrier index

d Distance

$E[\cdot] \quad$ Expectation operator

$E_{b} \quad$ Average electrical bit energy

$E_{s} \quad$ Average electrical symbol energy

$h(t) \quad$ Impulse response of the optical wireless channel

h Impulse response vector of the optical wireless channel

H MIMO channel matrix

$m \quad$ Time domain sample index in OFDM

$n(t) \quad$ AWGN at the receiver

$j \quad$ Imaginary unit, i.e. $j=\sqrt{1}$

$L \quad$ Number of symbols per transmission vector

$M \quad$ Modulation order

$N \quad$ Number of subcarriers, IFFT/FFT size

$N_{0} \quad$ Power spectral density of the AWGN

$P_{b} \quad$ Average electrical bit power

$P_{s} \quad$ Average electrical symbol power

$R_{s} \quad$ Symbol rate

$R_{b} \quad$ Bit rate

SE Spectral efficiency

S OFDM frame vector

$\widetilde{\mathbf{S}} \quad$ Distorted copy of the OFDM frame vector at the receiver

S Symbol vector 
$\widetilde{\mathbf{s}} \quad$ Distorted copy of the symbol vector at the receiver

$P_{t} \quad$ Average optical transmitter power

$\varrho \quad$ Photodiode responsivity

$t \quad$ Time variable

T Sampling period

n AWGN vector at the receiver

$x(t) \quad$ Biased information carrying signal

$x_{o}(t) \quad$ Bipolar OFDM signal

$\mathbf{x} \quad$ Biased information carrying signal vector

$y(t) \quad$ Received signal

y Received signal vector

$z \quad$ Height

$\mathbf{z}$ Angular detector axis vector

$\mathbf{R}_{\mathbf{z}} \quad$ Transformation matrix with respect to the $z$ axis

$\hat{\mathbf{z}} \quad$ Transformed vector $\mathbf{z}$

$\alpha \quad$ Reflection coefficient

$\delta^{2} \quad$ Gaussian noise (AWGN) variance

$\delta_{j s}^{2} \quad$ Thermal noise

$\delta_{d c}^{2} \quad$ Dark current noise

$\delta_{b q}^{2} \quad$ Background noise

$\delta_{s s}^{2} \quad$ Short noise

$q \quad$ Electron charge

$\xi \quad$ Proportionality constant in DC bias

$m_{s} \quad$ Smoothness of the reflecting material

$\sigma \quad$ Random parameter for TX look angle

f Specular reflected ray vector

$\mathbf{n}_{\mathbf{w}} \quad$ Normal vector to the wall

I Vector of a line connecting TX and RX

$\kappa \quad$ Lambertian order

$\pi \quad$ Number Pi, i.e. $\pi \approx 3.14$

$\exp (\cdot) \quad$ Exponential function

$N_{t} \quad$ Number of transmit antennas

$N_{r} \quad$ Number of available antennas at the receiver 
$I(t) \quad$ Intensity of the transmitted signal

$R_{l} \quad$ Load resistance

$r \quad$ Index of the receive antenna

$\Re \quad$ Real part of a complex variable

$|\cdot| \quad$ Absolute value

$\|\cdot\|^{F} \quad$ Frobenius norm

$Q(\cdot) \quad$ Q-function

$\Omega \quad$ Number of selected transmitting antennas in ASM

$\phi \quad$ Angle of emergence with respect to transmitter axis

$\Phi_{\frac{1}{2}} \quad$ Transmitter semiangle (at half power)

$\theta \quad$ Angle of incidence with respect to receiver axis

$\vartheta \quad$ Transformation angle around $z$ axis

$\varphi_{\frac{1}{2}} \quad$ Field of view (FOV) semiangle of receiver

$\omega \quad$ Random rotation angle of the receiver

$b_{m} \quad$ Bit assignment of signal vector $\mathbf{s}_{m}$

$I^{\mathrm{PAM}} \quad$ Intensity level of optical signal employing pulse amplitude modulation

$[\cdot]^{T} \quad$ Matrix/vector transpose

argmax Argument of the maximum

argmin Argument of the minimum

$\mathrm{d}_{\mathrm{H}}(\cdot, \cdot) \quad$ Hamming distance of two bit assignments

$\cos (\cdot) \quad$ Cosine function

$\sin (\cdot) \quad$ Sine function

$\ln (\cdot) \quad$ Logarithm to base $e$

$\log _{2}(\cdot) \quad$ Logarithm to base 2

$\log _{10}(\cdot) \quad$ logarithm to base 10

$P_{i} \quad$ Incident normalized unit power arriving at the wall

$P_{w} \quad$ Reflection intensity from the reflecting surface

$P_{r} \quad$ Optical power arriving at the receiver

$p(\cdot) \quad$ probability density function

$T_{h} \quad$ Throughput

$N_{b} \quad$ Number of bits in one symbol

$\gamma \quad$ Packet error probability

$P_{e} \quad$ Bit error probability

$k \quad \log _{2} M$ 
$E_{R X} \quad$ Received electrical energy

$R \quad$ Achieved data rate

$\mathbf{q}_{\mathbf{n}_{\mathbf{t}}} \quad$ A column of the channel matrix $\mathbf{H}$

Norm $n_{t} \quad$ Norm of $\mathbf{q}_{\mathbf{n}_{\mathbf{t}}}$

$h^{\prime} \quad$ Channel coefficients of the selected antennas in ASM

$G_{i} \quad i^{\text {th }}$ row of pseudo-inverse of channel matrix $\mathbf{H}$

$T X_{n}{ }^{\prime} \quad n^{\text {th }}$ image transmitter

$N_{T X_{n}{ }^{\prime}} \quad$ Number of light rays arriving at the receiver

$\phi T X_{n}{ }^{\prime} \quad$ Angle of emergence with respect to the image of transmitter $T X_{n}{ }^{\prime}$ axis

$\theta T X_{n} \quad$ Angle of incidence with respect to the receiver detector axis

$d_{T X_{n}{ }^{\prime}} \quad$ Distance between the image transmitter $T X_{n}{ }^{\prime}$ and receiver 


\section{Chapter 1 \\ Introduction}

\subsection{Motivation}

With the steady increase of wireless communications devices, there is a rapid growth of data demand, so that radio frequency (RF) spectrum (Figure 1.1) is becoming saturated and hence the limiting factor for achieving higher transmission rates [1,2]. The increasing functions and features of the mobile phones has led to the requirement for more bandwidth.

Current and future multimedia services such as web browsing, music and video downloads, video telephony, television on demand, making banking transactions, locating tourist attractions, identifying the best restaurants, social networking, remotely controlling the cooling, lighting, security in a home, and many other situations require higher data rates and thus enlarged transmission capacity. Also the global demand for data to and from mobile devices has more than doubled over the last few years and this trend is expected to continue [3], as shown in Figure 1.2.

The increasing number of wireless devices that are accessing mobile networks worldwide is one of the primary contributors to global mobile traffic growth. Each year several new different devices with increased capabilities and intelligence are being introduced in the market. Over half a billion (526 million) mobile devices and connections were added in 2013 [3]. Global mobile devices and connections grew to 7 billion in 2013, up from 6.5 billion in 2012. Globally, mobile devices and connections will grow to 10.2 billion by 2018 at a Compound Annual Growth Rate (CAGR) of $8 \%$ [3]. By 2018, there will be 8.2 billion hand-held or personal mobile-ready devices and 2 billion machine-to-machine (M2M) connections (e.g., GPS systems in cars, asset tracking systems in shipping and manufacturing sectors, or medical applications making patient records and health status more readily available, etc) [3,4]. Also, visual networking index (VNI) mobile forecast highlights that, in 2019 the mobile traffic per mobile connected end user device will reach 2,807 Mb per month up from $359 \mathrm{Mb}$ per month in 2014 global-wise [3]. To avoid device connection outage in the future, this increase in connections and traffic suggests the need for a complimentary spectrum resource in wireless communications. 


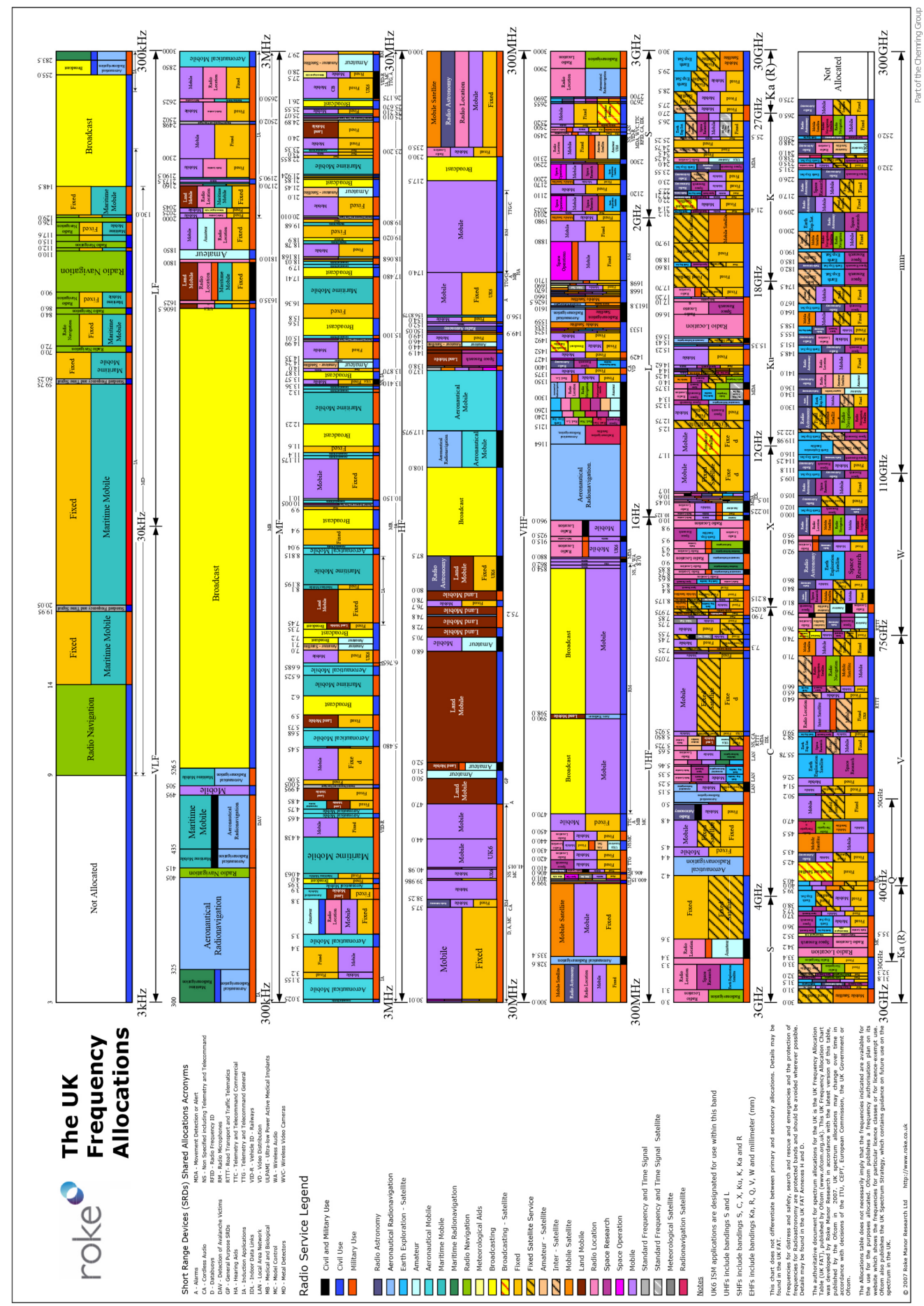

Figure 1.1: The UK Frequency Allocation [2] 


\section{Exabytes (EB) per Month}

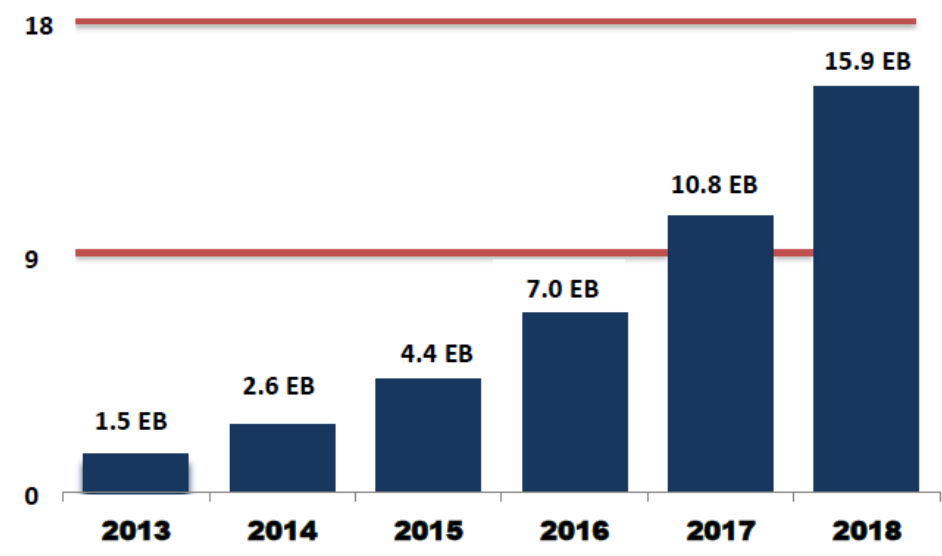

Source: Cisco VNI Mobile, 2012

Figure 1.2: Cisco Forecasts 15.9 Exabytes per Month of Mobile Data Traffic by 2018. Source: Cisco Visual Networking Index [3]

The spectrum ranging from infra-red (IR) to ultraviolet (UV) including visible light offers a huge bandwidth, which may be utilized for communications such as wireless local area networks (WLAN). The optical wireless communication (OWC) signal occupies wavelengths in the visible light spectrum from $380 \mathrm{~nm}$ to $750 \mathrm{~nm}$ and/or the near infra-red (NIR) spectrum from $750 \mathrm{~nm}$ to $2.5 \mu \mathrm{m}$. The total available bandwidth resource amounts to approximately 670 $\mathrm{THz}$, which is a factor of 10, 000 larger than the radio frequency (RF) spectrum $[5,6]$ and this can be clearly seen in Figure 1.3. In 2011, the institute of electrical and electronics engineers (IEEE) published a standard for VLC, the IEEE standard 802.15.7-2011, IEEE standard for Local and metropolitan area networks, part 15.7: short-range wireless optical communication using visible light [7].

In optical communication the transmitter modulates data and transforms the electrical signal to an optical signal, while the receiver demodulates signal and converts the incoming optical signal into an electrical current. OWC therefore promises to be a complimentary technology to RF communications. OWC is cheap because of the low cost and low number of required components in transmitting and receiving side compared to typical radio transceiver/access point. Again, with the development of solid-state lighting, white light emitting diodes (LEDs) could replace existing conventional light devices so communications and illumination can take place at the same time, hence saving power [8]. It is also safe to use in places where RF are 
not permitted e.g. underwater communication, the aviation industry, hospitals and healthcare facilities, and hazardous environments such as oil and gas refineries. OWC has the advantage of a license-free unregulated operation over a huge spectrum resource. Due to the fact that light does not propagate through opaque objects, the data communication is usually harder to intercept or to eavesdrop than RF signals. In Addition it is free of any health concerns as long as eye safety regulations are fulfilled $[1,9]$.

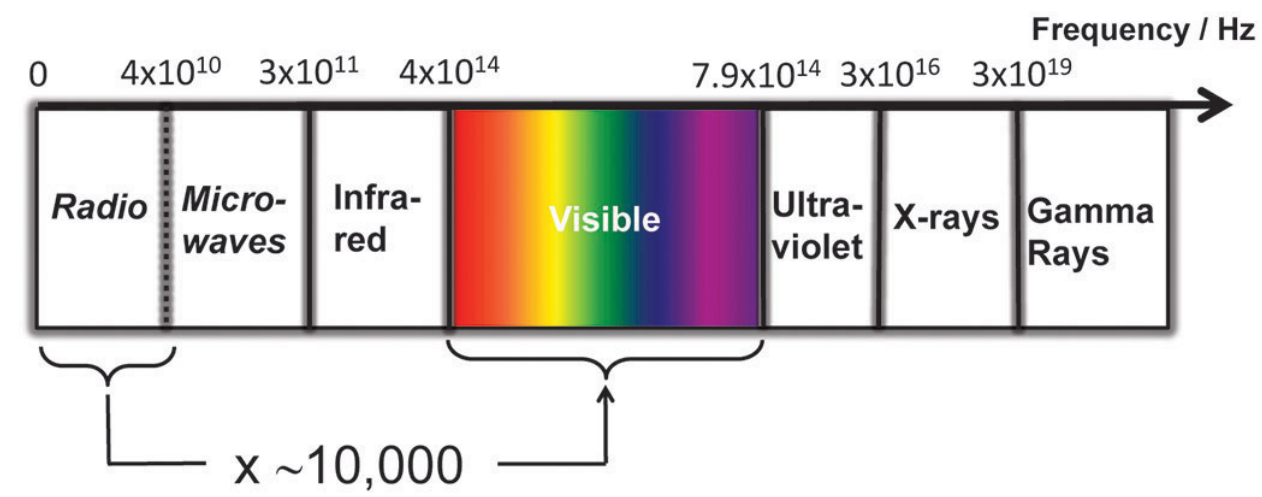

Figure 1.3: The electromagnetic spectrum and the vast potential of unused, unregulated, safe green spectrum in the visible light part. The visible light spectrum is 10,000 times larger than the entire radio frequency spectrum. Source: SPIE Connecting minds advancing lights [5]

The first recorded indoor OWC system was reported by Gfeller and Bapst in 1976 [10]. The system was capable of achieving 1 Mbps using on-off keying (OOK) at a centre wavelength of $950 \mathrm{~nm}$ in the infrared (IR) spectrum, employing diffuse radiation for a coverage of an office room. In 1996 Marsh and Kahn demonstrated an indoor diffuse OOK IR system with a data rate of 50 Mbps [11]. In 2000, Carruthers and Kahn presented a faster OOK IR system implementation with a data rate $70 \mathrm{Mbps}$ and a potential of up to $100 \mathrm{Mbps}$ [12]. Tanaka et al. first worked on white LEDs to convey information in addition to serving the main function of illumination in an indoor setup, later in 2003 they presented an OOK system providing 400 Mbps data rate [13]. In 2010, Vucic et al. demonstrated the potential of VLC systems with a 500 Mbps data rate [14]. Recently, at the University of Edinburgh, the research group led by Professor Harald Haas has presented a VLC orthogonal frequency division multiplexing (OFDM) system based on a single LED using Gallium Nitrate $\mu \mathrm{LED}$, where a data rate of 3 Gbps was achieved [15].

Multiple transmitter-receiver techniques have gained much attention in the field of RF communications, as they enable large spectral efficiencies and improve the performance of wireless 
systems. This parallel usage of multiple transmitters and receivers can also be employed in VLC e.g. OWC multiple input multiple output (OWC-MIMO) to enhance the overall system performance, improve spectral efficiency and reduce the bit error rate (BER) of a communication system [8].

In all the systems discussed above, the implementation faced several impairments including directionality i.e the signals can only be detected by a receiver which is placed within the emitted light beam. Hence, the propagation and the transmission range of optical signals can be restricted to specific spots or areas. Other impairments are the large size of a fixed receiver with an imaging lens which often becomes impractical in a real situation [8], high channel correlations for wide separations of transmitting and receiving optical devices [16], blockage of LOS propagation and coverage problems [17].

In this thesis, applications of optical OFDM, single carrier (SC) and MIMO techniques are studied and then some novel techniques are proposed to overcome some of impairments like directionality, channel correlation, interference, LOS beam obstruction and poor coverage. The introduced techniques can potentially enable indoor OWC with improved data rate performance.

\subsubsection{Contributions}

The aim of this thesis is to study, analyse and apply optical wireless transmission methods and techniques like OFDM, SC, MIMO and finally, develop novel techniques for system robustness and spectral efficiency in indoor environments. The following are the contributions of the thesis:

- Performance of asymmetrically clipped optical orthogonal frequency division multiplexing (ACO-OFDM), direct current biased optical OFDM (DCO-OFDM) and SC modulation schemes in single and multi-user systems is compared. Then the studied techniques were applied in a novel 4-Cells with narrow angular beams for indoor VLC system. This allows intensity modulated beams from light sources to be concentrated in specific areas to serve multiple mobile devices with low interference, and hence increase system capacity by the average factor of 2.7 times the single beam system capacity for both ACO-OFDM, DCO-OFDM and PAM. Also the channel capacity for 4-Cell beam system increases with the number of users to 4 times the single user capacity. The results of the studies have been presented and published in the Institute for Engineering and Technology (IET)- Intelligent Signal Processing Conference 2013 [18]. 
- Second, Angular diversity optical detectors in a MIMO configuration is developed so as to provide LOS channel conditions in more locations of the room and its performance is compared with state of the art vertical detectors. A novel adaptive form of spatial modulation (ASM) which is robust to rank deficient channels in different locations of the room is devised. Considering the same antenna separation of $0.6 \mathrm{~m}$ apart, ASM offers throughput improvement of average factor of 3.5 times SM perfromance in both vertical detectors and angular diversity detectors. Performance of MIMO methods operating over many room locations is compared. Angular diversity detectors offer a better throughput improvement than the state of the art vertical detectors, for example in ASM angular diversity receiver gives throughput of about 1.6 times that of vertical detectors. Also in Spatial Multiplexing (SMP) the angular detectors offer throughput about 1.4 times that of vertical detectors. SMP gives the best performance compared to RC, SM and ASM, for example SMP gives throughput about 2.5 times that of Repetition code (RC) in both vertical detectors and angular diversity receivers. Again SMP gives throughput about 6 times that of Spatial Modulation (SM) in both vertical detectors and angular diversity receivers. Also SMP provides throughput about 2 times that of ASM in both vertical detectors and angular diversity receivers. The results of the studies have been presented and published in IEEE Globe Telecommunications Conference (GLOBECOM 2014) [19].

- Third, a novel study of the impact of specular and diffuse reflections on MIMO performance is introduced. An optical MIMO system taking into account three scenarios: a LOS model, a Lambertian specular reflection model and a Lambert-Phong diffuse reflection model in both vertical and angular diversity receiver detectors is studied. Then performance statistics for MIMO methods operating over many room locations using AMC, ASM with an optical form of PARC is provided. The reflection models has shown a big impact in throughput improvement as the reflection coefficient value of the reflecting surface is increased. The diffuse reflection model show the best results as compared to LOS model, for example when the reflection coefficient $(\alpha)$ of 0.9 is considered and when the antenna separation is set to $0.6 \mathrm{~m}, \mathrm{RC}$ diffuse model shows throughput improvement of about 1.8 times that of LOS for both vertical detectors and angular diversity receivers. SM diffuse model shows throughput improvement of about 3 times that of LOS for both vertical detectors and angular diversity receivers. ASM diffuse model shows throughput improvement of about 2 times that of LOS for both vertical detectors and angular diversity receivers. SMP diffuse model shows throughput improvement of about 1.5 times 
that of LOS for both vertical detectors and angular diversity receiver. The results of the studies have been submitted to the IEEE Journal on Lightwave Technology for review.

\subsubsection{Thesis Outline}

The rest of the thesis is organized as follows: Chapter 2 gives an overview of the evolution and fundamentals of wireless communications where Radio frequency (RF) and OWC are explained. Wireless signal propagation and impairments are explained in detail, the variety of communication setups, the modulation formats and the existing literature on their capacity in the optical wireless channel are studied. The chapter also describes MIMO algorithms and operations, OFDM, O-OFDM and SC systems.

Chapter 3 presents the analysis and application of O-OFDM, single carrier (SC) in intensity modulation and direct detection (IM/DD). A 4-Cell narrow beam system is developed and O-OFDM, SC are applied to it in different scenarios. The performance of the 4-Cell narrow beams system and the single wide beam are compared in this section. The comparison between performance of single user single-beam light, single user multi-beam lights, and multi-beam multi-user scenarios is also described in this chapter.

Chapter 4 introduces adaptive spatial modulation (ASM) and angular diversity detectors in an indoor VLC MIMO system. The chapter discusses the angular receiver setup, MIMO techniques used and system throughput evaluation. The throughput results are compared at 10,000 room locations for different receiver detector orientation and transmitter separations.

Chapter 5 proposes the consideration of both LOS and reflections on the channel model. In this chapter specular and diffuse reflections are modelled in different scenarios. Different reflectivities in the room environment were considered. The total gain for either LOS and specular or LOS and diffuse channels are evaluated at the receiver detectors. To increase reception probability and combat the power loss caused by directivity issues, we again consider different MIMO configuration. The performance comparison between angular detectors and vertical detectors is evaluated in this chapter.

Finally, Chapter 6 concludes the thesis with the significant findings of this study. The limitations of the work are discussed, and an outlook with the future work is presented. 


\subsubsection{Chapter summary}

The chapter has highlighted the deficit in spectrum for current RF technology and pointed out the immense license-free spectral resource available for OWC systems. Some of the notable advances in the achievable data rates of current OWC system implementations have been presented in this Chapter. The main challenges in improving the information rates of the OWC systems considering the limitations of transmission channels have been introduced. In this context, methods to maximize the information rates of multi-carrier and single carrier OWC systems have been summarized as the main contributions of this thesis. The Chapter also concluded with the thesis outline. 


\section{Chapter 2 Background}

In this chapter, the background to wireless communications is provided. The chapter gives a brief overview of the history and evolution of wireless communications in Radio frequency (RF) as well as in optical communication. The chapter also discusses the wireless channel environment, link impairments and their relevance for enabling multiple-input multiple-output (MIMO) systems. The operation of OFDM and single carrier systems for both RF and optical communications along with their advantages and disadvantages are discussed. The chapter summarises challenges in optical communication and proposes novel approaches to minimize the challenges.

\subsection{Evolution of wireless communications}

The history of wireless communications stretches back many centuries. Many of the earliest systems were inherently line of sight using such techniques as smoke signals, flashing lights and semaphore. For example, the French had an elaborate, essentially countrywide, semaphore system (Figure 2.1), developed by Claude Chappe and consisting of series of relay stations $[20,21]$. Possibly, the first non-LOS systems were the drum signalling techniques used by tribes in Africa [22].

The understanding of the electromagnetic force is a founding pillar of modern telecommunications and there could be no wireless communications without this knowledge [22]. The idea of wireless communications is based on the mathematical analysis of by James Clerk Maxwell. His equations form the basis of computational electromagnetics and this are the foundation of wireless technology [23]. In 1880, Alexander Graham Bell demonstrated the first implementation of wireless system using light beams in form of photo-phone. He employed a mechanism of vibrating mirror at the transmitter to modulate a voice message onto a light signal [24-26]. In 1895, Guglielmo Marconi first demonstrated modern wireless technology, which also became known as radio [20,21,27]. The first such systems were in a sense digital since they used Morse code, which had been invented by Samuel Finley Breese Morse for use in telegraphy. A few years later, speech communication using analogy modulation came into applications. 
The potential of wireless communications was unlocked when the work of Claude Shannon was published. In 1948, Shannon opened up the field of field of information theory that characterised the limits of reliable communication [28]. He showed that there is a maximal channel capacity (achievable data rate) for which the error probability could be as low as desired. Further, communicating at higher data rates demands either more bandwidth or greater signal-tonoise-ratio (SNR) [29]. In fact, it was Shannon who established performance limits of practical modern communication systems. Since then, significant research has progressed into trying to approach the information theoretical channel capacity predicted in his work.

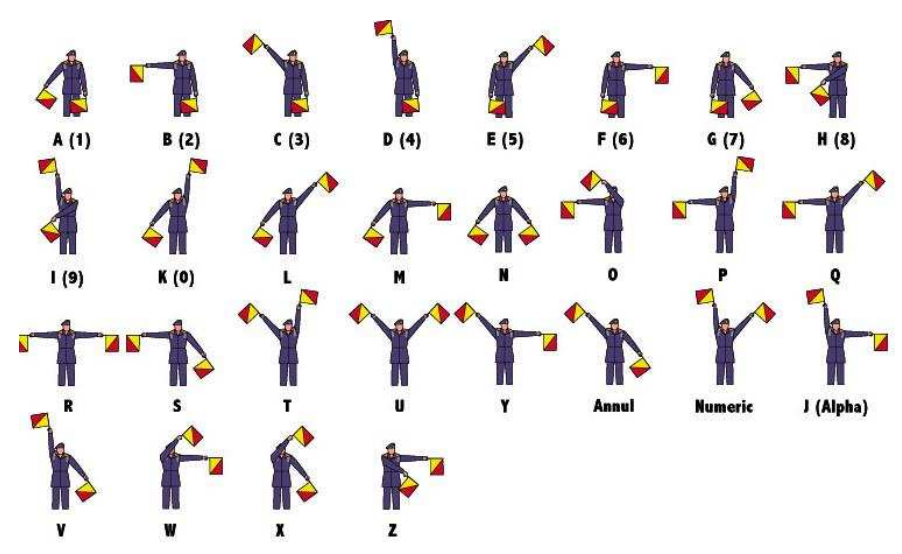

Figure 2.1: The semaphore signalling alphanumeric system[30]

The development of modern terrestrial wireless systems has been driven in large measure by the development of cellular radio systems [31,32]. The cellular principle introduced the concept of frequency reuse over large spatial domains. This leads to a very efficient use of the available radio spectrum and allows for a very large number of simultaneous users of a given frequency over a wide area. As a result the world is today moving to an untethered mobile wireless communications environment based on cellular-like system architectures. In 1979, a Japanese telecommunication company Nippon Telegraph and Telephone (NTT) became the first company to implement a commercial cellular mobile phone network in the world, followed by the Nordic Mobile Telephone (NMT) system that launched the similar system in Denmark, Norway, Sweden and Finland in 1981. Later in 1983 American Telephone and Telegraph (AT \& T) deployed the first cellular system in Chicago in 1983 [22,33]. AT \& T used an analogue transmission format and was completely saturated by 1984 , the developers having grossly underestimated the public appetite for mobile phone services. Since then there has been an almost explosive growth of cellular radio, and this continues today.

In the early 1990s the first digital cellular or second generation systems, which also called 
global system for mobile communications (GSM) appeared. These provided increased capacity and performance using digital transmission formats coupled with improved digital signal techniques and hardware platforms. In contrast to the first generation wireless systems, which differed from country to country, the second generation systems were developed in a collaborative manner. So, GSM became a robust, interoperable and widely accepted standard that combined expertise from various organisations under the guidance of the European Telecommunications Standards Institute. GSM gained such momentum that it was quickly adopted by many countries around the world. The digital nature of the GSM systems enabled data services to be incorporated and packet data services were introduced towards the end of the 1990s. The interoperability of GSM, along with its digital nature, provided mobile communications around the world and motivated the need for further, global co-operation services and technologies [34].

As the second generation wireless systems were being deployed across the world, research towards the third generation networks was on the way. The International Telecommunications Union (ITU) had started work on the evolution of the GSM standard in the 1980s. The GSM systems download speed of $14.4 \mathrm{~kb} / \mathrm{s}$ was insufficient for the rapidly growing data service demand. Based on that, research in the third generation systems targeted at achieving data rates of at least $2000 \mathrm{~kb} / \mathrm{s}$. To facilitate this technological advance, the 3rd Generation Partnership Project (3GPP) [35] was established so as to coordinate research activity across the world. $3 \mathrm{GPP}$ is an international organisation consisting of several international standardisation bodies from Europe, U.S.A., Japan, China and South Korea. In second generation systems time division multiple access (TDMA) or frequency division multiple access (FDMA) was used to facilitate multiple users, the third generation systems designed to use code division multiple access (CDMA). CDMA would support ultra wide bandwidth and would facilitate a large number of co-channel users, while remaining relatively immune to interference [36].

The increasing application of mobile devices has led to an exponential growth in the wireless data demands. The global mobile data demand is more than doubling over the last several years and expected to continue, as shown in Figure 1.2 of chapter 1. This leads to RF spectrum saturation, consequently a limit on achievable data rates. In an attempt to increase the spectral efficiency without more bandwidth or a greater SNR, researchers began to exploit the spatial domain. The idea of multiple spatial channels between two users is first considered in [37] where each channel is focused to operate with the same data rate. In this system the overall data 


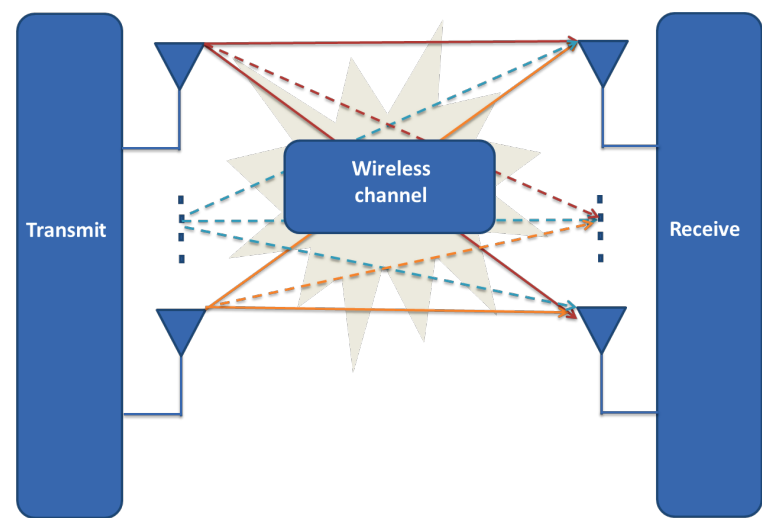

Figure 2.2: The MIMO system setup. The signal transmitted from each transmit antenna is detected by all receiving antennas

rate would increase linearly with the number of parallel connections between the transmitter and receiver. The idea was named the multiple-input multiple- output (MIMO) system. An example of a MIMO setup is shown in Figure 2.2 where the receiver detects multiple versions of the transmitted signal in the wireless environment. The new MIMO research outcomes were then incorporated for the first time in Release 7 of the 3GPP evolution standard and have remained an integral part since [34]. MIMO systems, along with OFDM, are considered as the backbone of the fourth generation wireless technologies led by the long-term evolution advanced (LTEA) initiative where peak data rates of up to $1 \mathrm{Gbit} / \mathrm{s}$ in the downlink and $450 \mathrm{Mbits} / \mathrm{s}$ in the uplink are possible. In particular, the employed technology is designed to be competitive for the next decade [38]. Aside from LTE-A, the IEEE 802.16 wireless access standard, referred to as WiMAX, also incorporates the use of MIMO systems [39]. In addition, MIMO systems are already part of the IEEE 802.11n and the latest IEEE 802.11ac standard for wireless local area networks and most modern wireless network cards are manufactured with the IEEE 802.11ac standard in [40].

As the wireless data demand increases, the spectrum resource also becomes a scarce commodity. This demand has driven research forward to find enhancements to existing RF wireless communications. The recent research activities have facilitated the evolution of optical wireless technology. The entire electromagnetic spectrum up to $300 \mathrm{GHz}$ is almost completely allocated $[1,2]$. Therefore, in order to cope with the increasing data demand for wireless communications, the light spectrum can be used. The optical spectrum is much larger than the microwave spectrum as shown in Figure 1.3. Also, the spectrum is mostly unused for wireless data transmission $[1,13]$. OWC is then highlighted as a potential solution to the global wireless spectrum 
shortage in [4].

The remainder of the chapter covers the basic overview of optical channel where, channel propagation properties, eye safety and optical wireless channel model are explained. It also gives a brief information about single carrier, multi-carrier and MIMO.

\subsection{Optical wireless channel link}

Wireless optical channels differ in several ways from conventional communications channels. In this section the building blocks of the transmitter and receiver front-ends are elaborated. The physical basis for the various amplitude and power constraints as well as propagation characteristics in indoor environments are also described. The geometry of a wireless communication scenario is defined by the position and transmission/reception characteristics of the transmitters and receivers in an indoor or outdoor environment together with some reflection properties of the objects in the setup. Based on the light propagation path radiated by the LEDs and detected by the Photo Diodes (PDs), there are two general link arrangements, i.e. LOS and NLOS communications [41].

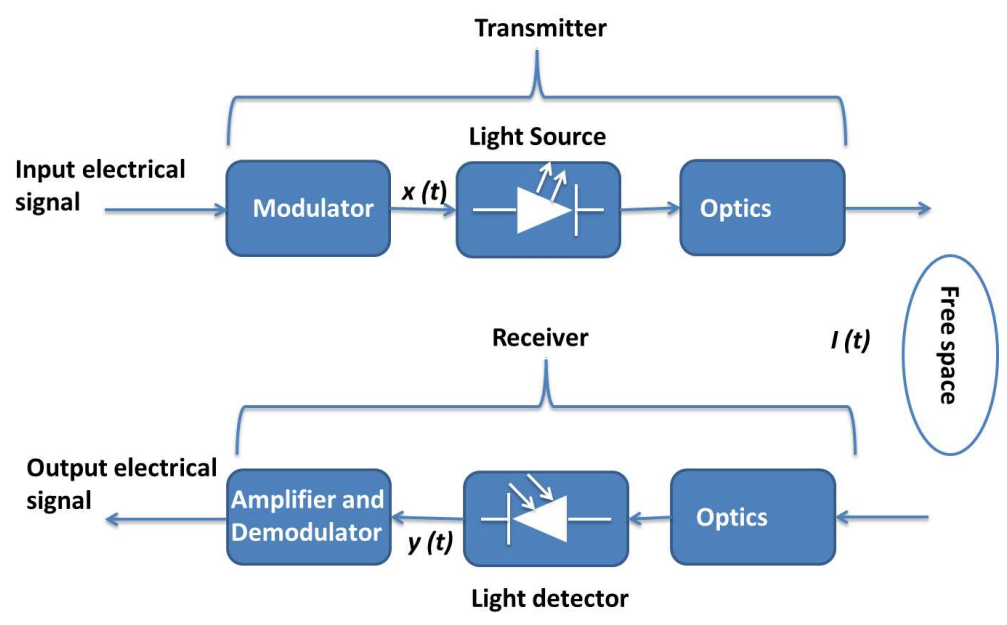

Figure 2.3: Block diagram of a typical optical wireless system

Many optical communication system are termed intensity modulation, direct detection format (IM/DD), this is a modulation scheme where the intensity of an incoherent optical source like LED is modulated and varied in accordance with the amplitude of the modulating signal. Demodulation in (IM/DD) is achieved through detection of the optical power and conversion using a photo-diode [41]. However some of optical systems employ coherent light sources like laser 
where intensity can be varried in accordance with either amplitude, phase or frequency of the modulating signal. Detection in this case can be performed using heterodyne or homodyne downconversion by a local-oscillator (LO) laser, followed by the appropriate electrical-domain detector [41]. Figure 2.3 presents a schematic of a simplified free-space intensity modulated, direct-detection optical link. The optical intensity of a source is defined as the optical power emitted per solid angle in units of Watts per steradian [42]. In Figure 2.3 the transmitter unit consists of a digital modulator which is responsible for the modulation of the input electrical signal into a current signal $x(t)$. The current drives LED/laser diode (LD) or alternatively an array of LEDs/lasers. Here, the information-carrying current signal is transformed into optical intensity. The optical signal can be passed through an optical system for further shaping of the transmitted light beam. In this optical system, a collimator, an optical amplifier lens, or a diffusor can be employed to narrow or broaden the light beam $[1,25]$. The optical signal is then transmitted over the optical wireless channel (free space). The information sent on this channel is not contained in the amplitude, phase or frequency of the transmitted optical waveform, but rather in the intensity of the transmitted signal $I(t)$ [26]. Some of the optical energy is absorbed by the objects in the environment, and the rest is reflected back into the atmosphere in a diffuse or specular fashion [43]. In general LOS and NLOS signal components both arrive at the receiver.

At the receiver, an optical filter [44] can be applied to select a portion of interest in the light spectrum or can be used to reduce the interference from ambient light. So, the optical signal is passed through a system of optical elements, e.g. lenses and collimator, to amplify its strength and to align the incoming light according to the photodetector's properties $[1,41,45-48]$. At the photodetector, i.e. a PD or again an array of PDs, the optical signal is converted back to electrical current $y(t)$. The current signal is amplified by a transimpedance amplifier (TIA), and a digital signal processing (DSP) is employed for demodulation of the information bits. This electro-optical conversion process is termed optical intensity modulation and is usually accomplished by LED or LD operating in the $850-950 \mathrm{~nm}$ wavelength band $[26,41]$. The information bearing intensity signal which is transmitted must remain positive for all time since the transmitted power can physically never be negative, i.e.,

$$
(\forall t \in \Re) I(t) \geq 0
$$


Kahn and Barry provide a common baseband channel model for OWC in [41] as:

$$
y(t)=g(t) * I(t)+n(t)
$$

where $y(t)$ is the received waveform, $g(t)$ is the channel impulse response and $I(t)$ is the instantaneous transmitted optical power. The symbol $*$ denotes the convolution operator and the noise is represented by $n(t)$. The term $n(t)$ is assumed to be the sum of shot noise due to intense ambient light and thermal noise. Two main noise sources are considered in OWC systems. Shot noise is caused by background light sources like the sun and additional illumination devices which are not for data transmission. This ambient light is dominant compared to the signal generated noise. This ambient noise can be modelled as zero mean AWGN which is independent of $I(t)[41,47]$. Thermal noise is induced in the receiver electronics like the preamplifier of the receiver front-end. It is independent of $I(t)$ and independent of the shot noise. Therefore, $n(t)$ can be modelled as real valued AWGN with zero mean and a variance

$$
\delta^{2}=\delta_{\mathrm{ss}}^{2}+\delta_{\mathrm{js}}^{2}
$$

where $\delta_{\mathrm{ss}}^{2}$ is the shot noise variance and $\delta_{\mathrm{js}}^{2}$ is the thermal noise variance $[41,47]$. The noise power is given by $\delta^{2}=N_{0} B$, where $N_{0}$ is the noise power spectral density and $B$ is the bandwidth. As for IM/DD based OWC the transmitted signal represents optical power, $I(t)$ has to be non negative, and thus $I(t) \geq 0$ holds. This is in contrast to RF communications where the average transmitted power depends on $|I(t)|^{2}$ which is appropriate when $I(t)$ represents amplitude. The average transmitted power $P_{t}$ in OWC is therefore given by

$$
P_{t}=\lim _{T \rightarrow \infty} \frac{1}{2 T} \int_{-T}^{T} I(t) d(t)
$$

where $T$ is the period for transmitted light wave and the average received optical power can be written as

$$
P_{r}=h P_{t}
$$

where $P_{r}$ represents the optical received power and $h$ represents the channel gain of the optical 
link. As further discussed in Section (2.2.3) intensity modulated optical links can be represented by the direct-current (DC) channel gain $h$ due to the flat frequency response of the indoor short range optical wireless channel. In indoor short range OWC channel the delay spread is very small as a result, the bandwidth of the signal becomes very smaller than the coherence bandwidth.

\subsubsection{Channel propagation properties}

As in radio frequency transmission systems, multipath propagation effects are important for OWC networks. The power radiated from the transmitter may take many reflected and refracted paths before arriving at the receiver. In radio systems, the sum of the transmitted signal and its images at the receiver antenna cause spectral nulls in the transmission characteristic. These nulls are located at frequencies where the phase shift between the paths causes destructive interference at the receiver. This effect is known as multipath fading [49].

Unlike RF systems, multipath fading is not always a major impairment in optical wireless communications. The receiving antenna in an OWC is the light detector which typically has an active radiation collection area of approximately $1 \mathrm{~cm}^{2}[1,13]$. The relative size of this antenna with respect to the wavelength of the light wave is immense, on the order of $10^{4} \lambda$. The multipath propagation of light produces fade condition in the amplitude of the received electromagnetic signal at spacings on the order of a half wavelength apart. The light detector integrates the square of the amplitude of the electromagnetic radiation falling on it [50]. The size of the photodetector with respect to the wavelength of the light inherently provides spatial diversity in the receiver which mitigates the impact of multipath fading $[26,50]$. The impact of multipath dispersion is noticeable in diffuse OWC communication systems, but in short distance line-of-sight (LOS) links, multipath dispersion is seldom a significant issue [51].

\subsubsection{Eye Safety}

The design of the transmitter unit is subject to eye safety regulations. Safety considerations must be taken into account when designing a wireless optical link. Since the energy is propagated in a free-space channel, the impacts of this radiation on human safety must be considered [41]. There is number of international standards bodies which provide guidelines on LED and laser emissions namely: the BSI British Standards-BS EN 62471 : 2008, the International Electrotechnical Commission (IEC) (IEC60825-1), American National Standards Institute (ANSI) 
(ANSI Z136.1), European Committee for Electrotechnical Standardization (CENELEC) among others [26].

In [25], it is explained that incoherent diffuse continuous-wave-modulated LEDs belong to the exempt group classification and pose no photobiological hazard for the human eye. This is according to the BS EN 62471 : 2008 standard for photobiological safety of lamps and lamp systems. It is appropriate if the irradiance does not exceed $100 \mathrm{~W} / \mathrm{m}^{2}$ at a distance of $0.2 \mathrm{~m}$ from the optical source in the direction of maximal directivity within 1000s. [52]

Generally, a single LED has a generalized Lambertian radiation pattern [10,41]. It has been shown in [43] that incoherent diffuse LEDs, e.g. the Vishay TSHG8200 or the OSRAM LCW W5SM Golden Dragon, are inherently designed in accordance with the eye safety standard, and there is significant room for intensity amplification through an optical system of lenses and collimators $[53,54]$.

\subsubsection{Optical wireless channel model}

Optical wireless link models in general are given in [1] and Figure 2.4 and for channel gains calculations were given in $[41,48]$. The frequency response of the optical channel is flat near DC and the channel has the low-pass characteristic because of direct detection. The authors describe the DC channel gain as the most distinctive parameter for describing an intensity modulated optical link. The channel gain $h$ affects the optical power received by the receiver by inducing a specific path loss as given by equation (2.5). The optical path loss is given by $-10 \log _{10} h$, whereas the electrical path loss is given by $-10 \log _{10} h^{2}$. Kahn and Barry describe three link models depending on the geometry: LOS links, directed NLOS links and diffuse NLOS links. Figure 2.5 presents all the three geometries. 


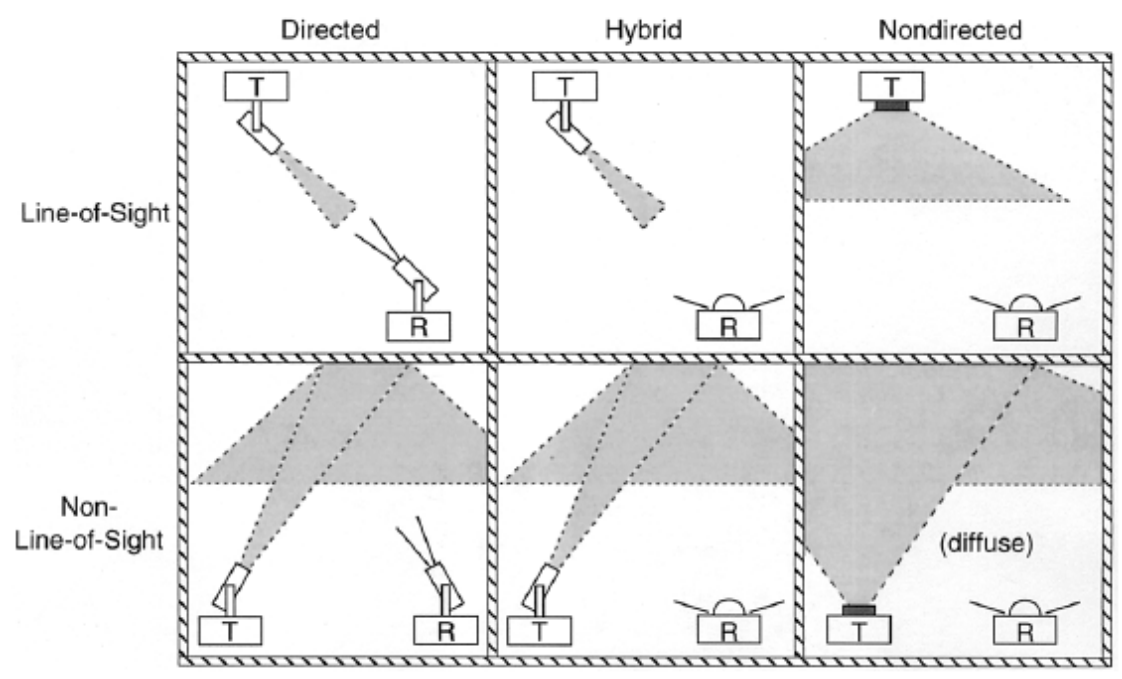

Figure 2.4: Classification of simple indoor OWC links according to the degree of directionality of the transmitter and receiver [41]

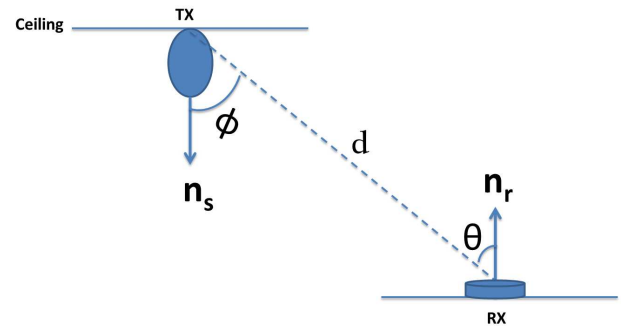

(a)

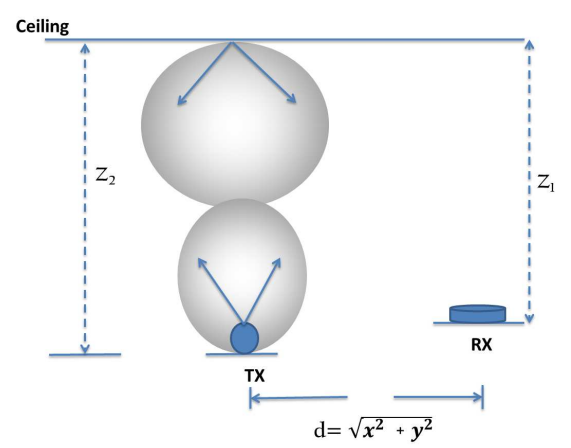

(c)

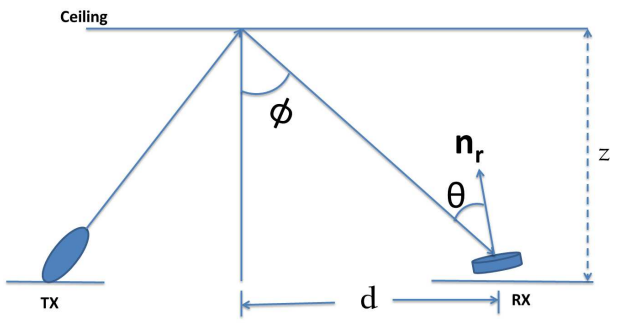

(b)

Figure 2.5: Geometries used in channel gains calculations (a) LOS link (b) Directed non-LOS link (c) Non-directed non-LOS link

Figure 2.5 (a) depicts the LOS link, where $\phi$ is the angle of emergence with respect to the transmitter (TX) axis and $\theta$ is the angle of incidence with respect to the receiver (RX) axis. 
The distance from the transmitter to the receiver is represented by the variable $d$. Based on the analysis made in [41], the channel gain of this LOS link can be calculated as follows: $[6,41]$

$$
h=\left\{\begin{array}{cc}
\frac{A(\kappa+1) \cos ^{\kappa}(\phi) \cos (\theta)}{2 \pi d^{2}}, & 0 \leq \theta \leq \varphi_{\frac{1}{2}} \\
0, & \theta>\varphi_{\frac{1}{2}}
\end{array}\right.
$$

where,

$$
\kappa=\frac{-\ln (2)}{\ln \left(\cos \left(\Phi_{\frac{1}{2}}\right)\right)}
$$

Scalar $A$ is the collection area of the detector, $\Phi_{\frac{1}{2}}$ is the transmitter semi-angle (at half power) and the scalar $\varphi_{\frac{1}{2}}$ is the field of view (FOV) semi angle of the receiver. The channel gain $h$ depends on the specific position of the transmitter and the receiver. If a receiver and a transmitter are not in each others FOV, $h=0$ holds.

Figure 2.5 (b) illustrates directed non-LOS. In the figure, both the transmitter and the receiver axes are directed towards the ceiling. For this setup, it is assumed in [41] that the diameter of the spot of illumination at the ceiling is smaller than the distance $d$ between the spot and the receiver. If the ceiling at a height of $z$ (with respect to the receiver position) has reflectivity of $\alpha$, the channel gain can be calculated as follows: $[6,41]$

$$
h=\left\{\begin{array}{cc}
\frac{\alpha A z}{\pi\left(z^{2}+d^{2}\right)^{\frac{3}{2}}} \cos (\theta), & 0 \leq \theta \leq \varphi_{\frac{1}{2}} \\
0, & \theta>\varphi_{\frac{1}{2}}
\end{array}\right.
$$

The last scenario discussed by Kahn and Barry is a non directed-LOS link. Figure 2.5 (c) illustrates this type of link. In [41] it is assumed that the transmitter at coordinate $(0,0)$ is pointed straight upward and emits a Lambertian pattern. It is also assumed that the receiver at coordinate $\left(x_{2}, y_{2}\right)$ is pointed straight upward. Both the transmitter and receiver are located in the horizontal $(x, y)$ plane, where the distance from the ceiling to the transmitter and receiver is $z_{1}$ and $z_{2}$ respectively. The optical power reflected from the ceiling is integrated at the receiver position resulting in: $[6,41]$ 


$$
h=\frac{\alpha A z_{1}^{2} z_{2}^{2}}{\pi^{2}} \underset{\text { ceiling spot area }}{\left.\iint_{1}+x^{2}+y^{2}\right)^{2}\left[\left(z_{2}^{2}+\left(x-x_{2}\right)^{2}+\left(y-y_{2}\right)^{2}\right]^{2}\right.}
$$

\subsection{Single-carrier and multi-carrier modulation}

Single-carrier pulse modulation is one method of encoding information either in the position of the pulse i.e. $M$-PPM, in the amplitude of the pulse i.e. $M$-PAM or in the width of the pulse $M$-PWM. In multi-carrier modulation, the information is split and encoded in several complex components i.e. $M$-QAM and then transmitted in separate sub-carriers signals. During the multi-carrier modulation process, the inverse FFT (IFFT) is used as a multiplexing technique at the transmitter, and the FFT is used as a demultiplexing technique at the receiver [25]. Figure 2.6 illustrates the frequency spectra of single carrier Figure (2.6 (a)) and of multi-carrier Figure (2.6 (b)) transmissions.

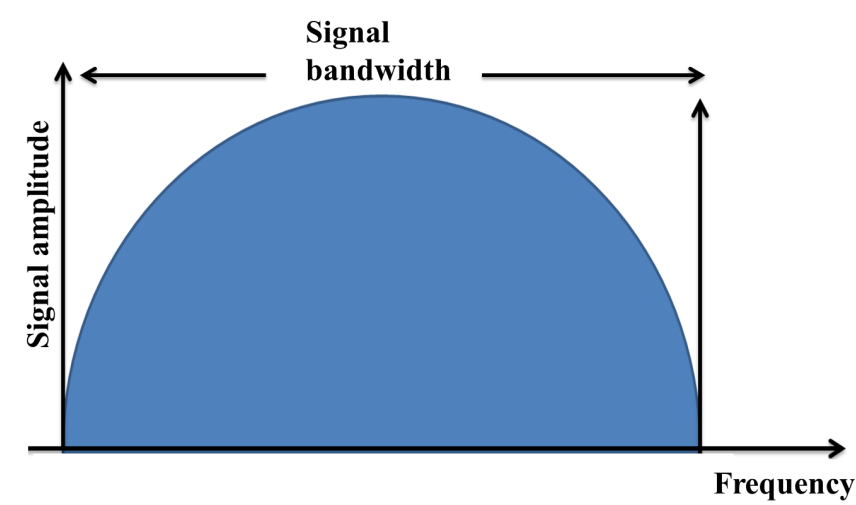

(a)

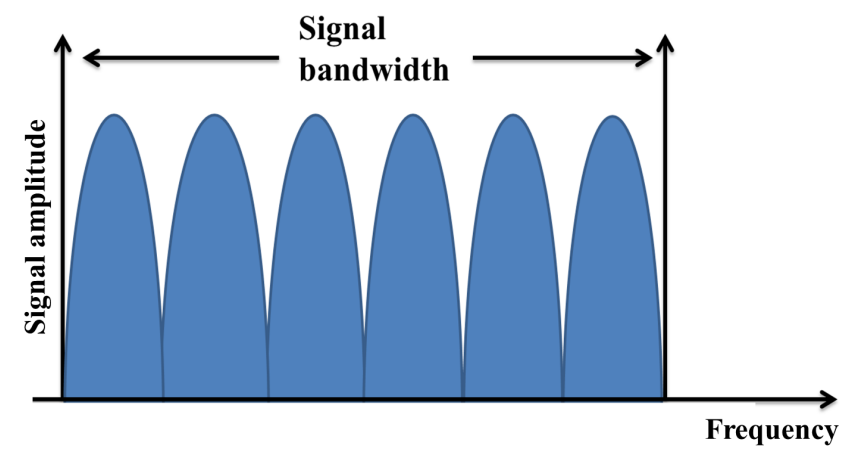

(b)

Figure 2.6: Illustration of frequency spectra formats of (a) Single carrier and (b) Multi-carrier 


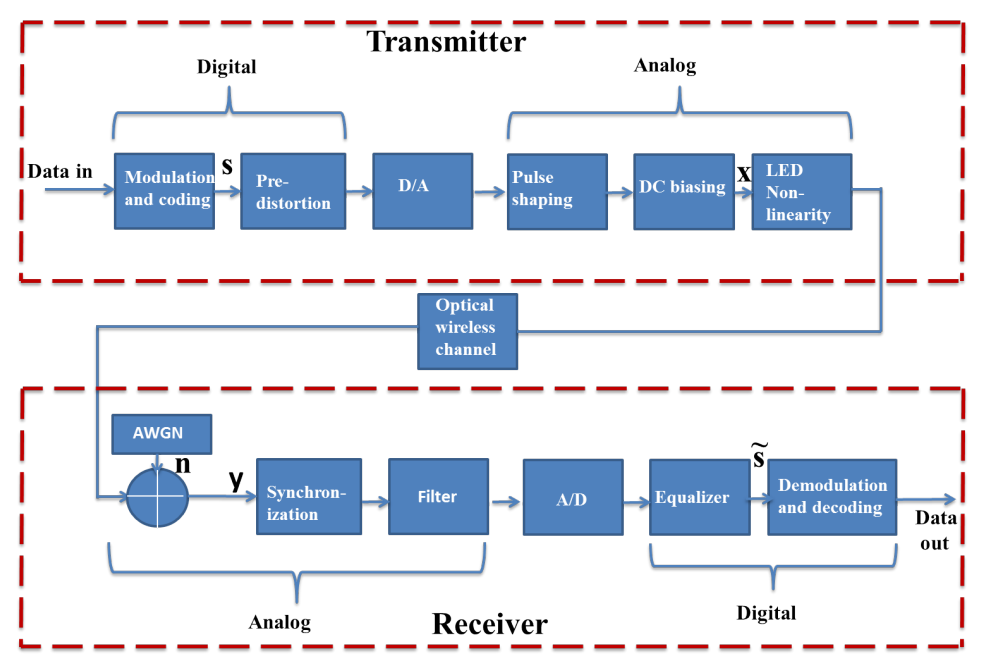

Figure 2.7: Block diagram of single-carrier transmission in OWC using PAM and PPM

\subsection{1 $\quad M$-PPM}

Figure 2.7 presents a block diagram of single-carrier transmission with pulse modulation. Pulse-position modulation (PPM) is a form of signal modulation in which $M$ message bits are encoded by transmitting a single pulse in one of possible time-shifts. In $M$-PPM, $\log _{2}(M)$ bits with equal probability form a time domain symbol. It consists a sequence of $M$ time slots as in Figure 2.8, where one slot has a current level of $\sqrt{M P_{s}}$, and the other $M-1$ slots are set to zero. Here, $P_{s}$ denotes the average electrical power of the $M$-PPM symbol and this is related to the average electrical bit power by: $P_{s}=P_{b} \log _{2}(M)$. The respective energy per symbol $E_{s}$ and energy per bit $E_{b}$ are obtained in the following relation: $E_{s}=P_{s} / R_{s}$ and $E_{b}=P_{b} / R_{s}$. $M$ time slots each with duration $T / M$ fit within a time period of $T$. Therefore, the $M$-PPM symbol with a double-sided bandwidth of $B=M / T$ has a duration of $T$ for a symbol rate of $R_{s}=B / M$, and it is grouped in the train of $L$ symbols, $\mathbf{s}$ [25]. To determine the spectral efficiency of a modulation scheme, the number of bits that can be transmitted per symbol duration and per symbol bandwidth should be evaluated. So, the spectral efficiency $\mathrm{SE}=R_{b} / B$, where $R_{b}=\log _{2}(M) R_{s}$ is the bit rate. Thus, the spectral efficiency of $M$-PPM can be expressed as $\mathrm{SE}=\log _{2}(M) / M$ bits/s/Hz $[25,41,55]$.

Since the train of symbols, $\mathbf{s}$, is affected by the distortion caused by the inverse of the non-linear transfer function of the LED transmitter, the symbol is scaled in order to fit within the front-end optical power constraints. The signal is then passed through a digital to analog converter (D/A) so that the train of digital signals can be converted into a train of continuous-time pulses. A 


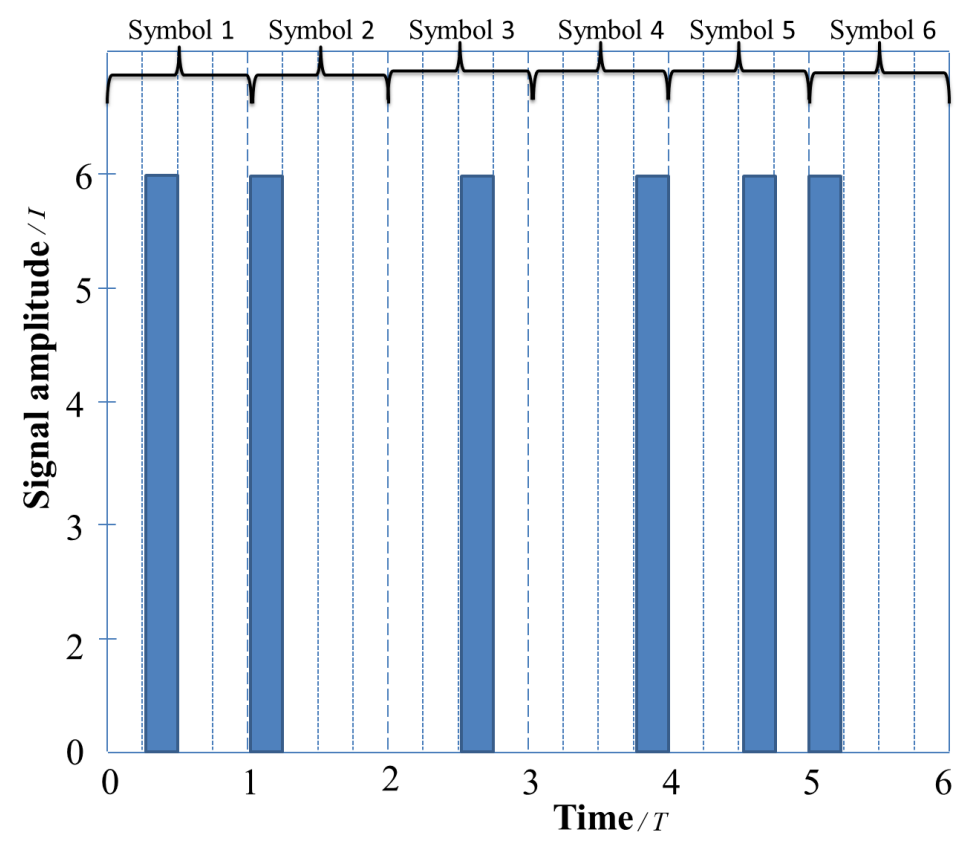

Figure 2.8: Illustration of 4-PPM

pulse shaping filter is applied to obtain a band-limited signal. In $M$-PPM a DC bias level is not needed since the information-carrying pulse has an optical power greater than minimum optical power constraints.

The signal is transmitted through the optical wireless channel and is then distorted by AWGN $\mathbf{n}$ at the receiver during recovery of the received signal $\mathbf{y}$. The received signal is synchronized and then passed through a filter matched to the impulse response of the pulse shaping filter at the transmitter. The signal is sampled at a frequency of $M / T$ at the $\mathrm{A} / \mathrm{D}$ converter $[25,41,55]$. The signal is equalized to correct the channel effects and then the distorted copy of the transmitted symbol $\widetilde{\mathbf{s}}$, is obtained. A hard-decision or soft-decision decoder can be employed to obtain the received bits.

\subsection{2 $M$-PAM}

The block diagram in Figure 2.7 presents single-carrier transmission with $M$-PAM. In this case, $\log _{2}(M)$ input bits having equal probability form a time domain symbol with a double-sided bandwidth of $B=1 / T$ and a duration of $T$ for a symbol rate of $R_{s}=B$. The symbols are then assigned to current levels of $\varsigma \sqrt{\frac{3 P_{s}}{(M-1)(M+1)}}, \varsigma= \pm 1, \pm 3, \ldots . . \pm M-1$, and $P_{s}$ denotes symbol electrical power. 


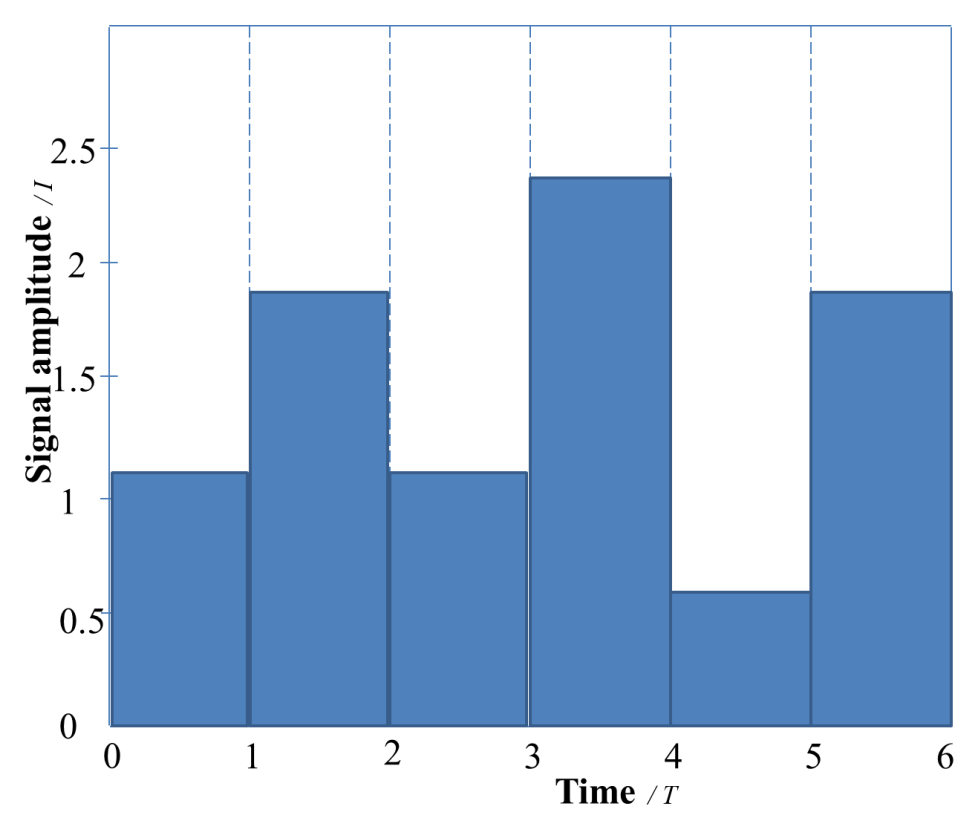

Figure 2.9: Illustration of 4-PAM

These levels are grouped in the train of $L$ symbols s. Here, $E_{b}=P_{b} / B=P_{s} / \log _{2}(M) B$ where $E_{b}$ and $P_{b}$ are energy per bit and power per bit respectively. Therefore the spectral efficiency of $M$-PAM is $\log _{2}(M)$ bits/s/Hz [25,41,55]. Since the train of symbols is pre-distorted, they are scaled and passed through the D/A converter then a pulse shaping filter is applied. Since at this stage symbols are bipolar, they require a DC bias, $B_{D C}$, to fit within the positive-only waveforms that can be transmitted by the LED. An example of 4-PAM is shown in Figure 2.9. The signal $\mathbf{x}$ is transmitted over the optical wireless channel, and at the receiver side the signal is distorted by AWGN, $\mathbf{n}$. Then received signal, $\mathbf{y}$, is synchronized and passed through a matched filter. The signal is sampled at a frequency of $1 / T$ at the A/D converter $[25,41,55]$. Next, the signal is equalized, and the distorted copy of the transmitted symbol $\widetilde{\mathbf{s}}$, is obtained and decoded to recover the transmitted bits.

In BER performance of PPM, lower modulation level requires more SNR than the higher modulation level can require. As the modulation level is increased, PPM becomes more power efficient and provides better BER performance. In contrast to $L$-PPM, $M$-PAM scheme requires more amount of power to achieve the same amount of BER that PPM can achive. However PAM is more bandwidth efficient compared to PPM $[47,56]$. 


\subsubsection{Optical OFDM using $M$-QAM}

In OFDM the total signal bandwidth in classical parallel data system can be divided into $N$ non-overlapping frequency sub-carries. The carries must be mathematically orthogonal to each other i.e the dot product of adjacent carriers should be equal to zero [57]. Also orthogonality can be viewed from the perspective of stochastic processes that, if two random processes are uncorrelated then they are orthogonal. So despite of overlapping sub-carriers, orthogonality eliminates inter-carrier interference (ICI) and therefore the energy from other sub-carrier does not contribute to another. Each sub-carrier is modulated with a separate symbol and then the $N$ sub-channels are frequency multiplexed [57]. The block diagram in Figure 2.10 presents multi-carrier O-OFDM transmission. The O-OFDM transmission can be implemented through two main techniques: the bipolar DCO-OFDM with a DC bias or the unipolar ACO-OFDM $[25,58]$.

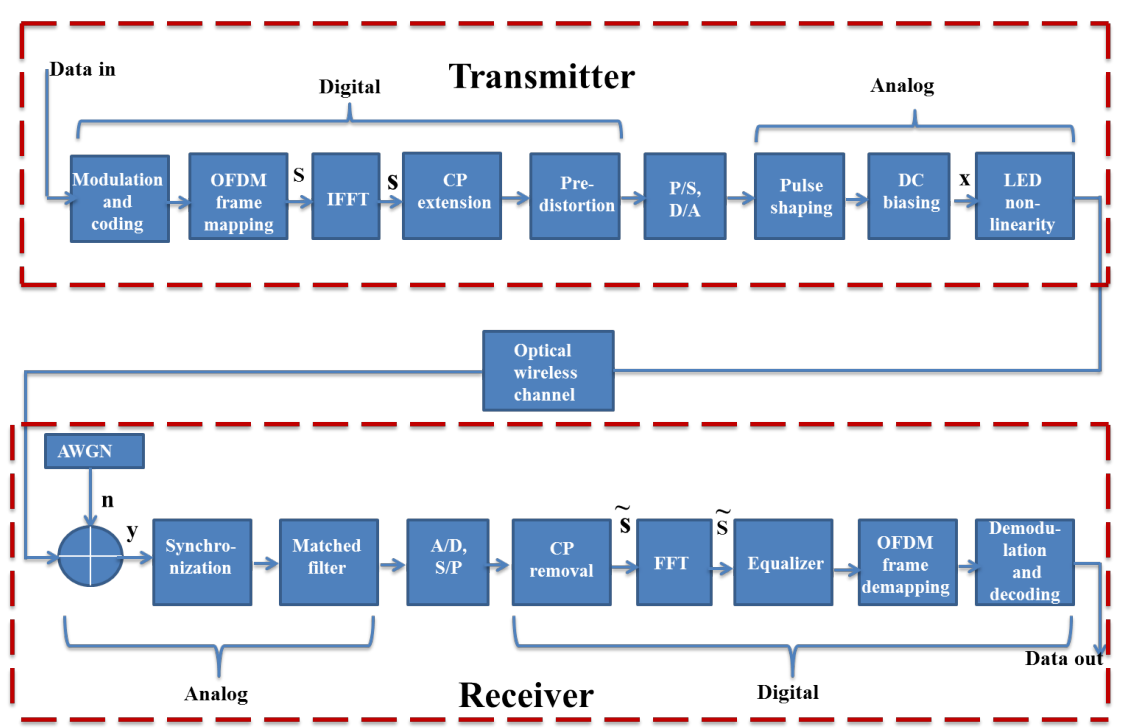

Figure 2.10: Block diagram of multi-carrier transmission using OFDM

In OFDM, the OFDM frame $\mathrm{S}$ is formed by $N$ subcarriers. The OFDM frame, $\mathrm{S}_{i, c}$, corresponds to the $i^{\text {th }}$ OFDM symbol, where $c=0,1, \ldots, N-1$, is the subcarrier index. Each subcarrier in a total OFDM frame double-sided bandwidth of $B=1 / T$, occupies a bandwidth of $1 / N T$. The two O-OFDM systems utilize a different portion of the available bandwidth. In OWC, the signal to be transmitted should be real and positive. To ensure a real-valued time domain signal, Hermitian symmetry should be imposed on the OFDM frame for both techniques, and the subcarriers with indices $c=\{0, N / 2\}$ are set to zero [25,58,59]. In DCO-OFDM, only $N / 2-1$ subcarriers in the first half of the frame, $c=1, \ldots, N / 2-1$, carry information symbols 
while in ACO-OFDM, even carriers are disabled and only the odd subcarriers are active. So far ACO-OFDM, only the $N / 4$ subcarriers with indices $c=1,3,5, \ldots, N / 2-1$ carry information symbols. The OFDM symbol s, is obtained by the IFFT of the OFDM frame S. In O-OFDM, the FFT is utilized as a multiplexing technique at the transmitter, as follows:

$$
\mathbf{s}_{i, c}=\frac{1}{\sqrt{N}} \sum_{c=0}^{N-1} \mathrm{~S}_{i, c} \exp \left(\frac{j 2 \pi m c}{N}\right)
$$

where $j=\sqrt{-1}$, and $m=0,1, \ldots, N-1$, denotes the time domain sample index within the OFDM symbol. Variable $\mathrm{S}_{i, c}$ can be complex (e.g. 16-QAM) unlike PAM or PPM. In addition to $\mathbf{s}_{i, c}, N_{C P}$ samples from the end of each OFDM symbol are appended at the beginning of the symbol to form the cyclic prefix (CP) extension, for mitigation of the inter-symbol interference (ISI) and the inter-carrier interference (ICI) $[25,59,60]$. If the number of subcarriers is sufficiently large, when added with $\mathrm{CP}$, they together transform the optical wireless channel from being potentially dispersive into a flat fading channel over the subcarrier bandwidth. This transformation reduces the computational complexity of the equalization process at the receiver to a single-tap equalizer [60]. As a result, the time domain OFDM symbol with CP occupies a double-sided bandwidth of $B=1 / T$, and it has a duration of $\left(N+N_{C P}\right) T$. For FFT sizes greater than 64 the time domain samples for the train of OFDM symbols closely matches a Gaussian distribution with zero mean and variance of $\delta^{2}[25,59]$.

To compensate the non-linear transfer characteristic of LED transmitter, pre-distortion using the inverse of the non-linear transfer function is applied [61]. Then the pre-distorted OFDM symbol is passed through a parallel-to-serial $(\mathrm{P} / \mathrm{S})$ block, and and then through the D/A converter to obtain the continuous-time signal. A pulse shaping filter is applied. To ensure non-negativity, since only the odd subcarriers are enabled in ACO-OFDM, the negative part of the ACO-OFDM symbol is clipped to zero without loss of information at the received odd subcarriers. The bipolar DCO-OFDM symbol is converted to a unipolar signal by adding direct current (DC) bias. The signal is DC-biased by a current $B_{D C}$ in the analog circuitry, and it is transmitted by the LED.

The signal $\mathbf{x}$ is transmitted over the optical wireless channel and when reaches the receiver it is distorted by AWGN, $\mathbf{n}$. The received signal, $\mathbf{y}$ is then synchronized, and passed through a matched filter. The signal is sampled at a frequency of $1 / T$ by the $\mathrm{A} / \mathrm{D}$ converter $[25,59,60]$. After serial to parallel (S/P) conversion, the CP extension of each OFDM symbol is removed 
in order to extract the distorted copy of the OFDM symbol, $\widetilde{\mathbf{s}}$ and then the signal is then passed through FFT process block. Here, the signal is converted back to the frequency domain. The FFT demultiplexing is carried as follows:

$$
\widetilde{\mathbf{S}}_{i, c}=\frac{1}{\sqrt{N}} \sum_{n=0}^{N-1} \widetilde{\mathbf{s}}_{i, c} \exp \left(\frac{j 2 \pi m c}{N}\right)
$$

where, $\widetilde{\mathbf{S}}_{i, c}$ and $\widetilde{\mathbf{s}}_{i, c}$ denote the distorted copy of the OFDM frame and symbol respectively.

\subsection{Multiple Input Multiple Output (MIMO)}

Multiple Input Multiple Output (MIMO) techniques have gained much attention in the field of RF communications as they enable large spectral efficiencies and improve the performance of wireless systems [62-66]. Therefore, these techniques are an appropriate way to utilize the available bandwidth efficiently. In addition to conventional data transmission in the time and frequency domain, MIMO techniques exploit the spatial domain for simultaneous data transmission. The benefits of these techniques rely on multipath signal propagation and on the fading effects of wireless channels caused by reflection, diffraction and scattering. These effects induce characteristic "spatial signatures" of the multiple wireless links which allow the communication receiver to separate out signals from different transmitters to achieve better performance [6].

The idea behind spatial multiplexing is that a source stream is split into several substreams as in Figure 2.2. Each substream is then transmitted from a different transmitter. In this case, all the transmitters are active at the same time and each receiver uses multiple antennas to detect the multiple co-channel signals. For the facilitation of detection, a rich scattering environment is very important. Therefore, the receiver exploits the different channel fingerprints of each co-channel signal to separate them. The substreams are then demodulated and recombined to obtain the original source signal. The channel gain matrix for a MIMO system with $N_{t}$ transmitters and $N_{r}$ receivers can be expressed as follows: 


$$
\mathbf{H}=\left(\begin{array}{ccccc}
h_{11} & h_{12} & \cdot & \cdot & h_{1 N t} \\
h_{21} & h_{22} & & \cdot \\
\cdot & & \cdot & \cdot \\
\cdot & & & \cdot & \cdot \\
h_{N r 1} & \cdot & \cdot & \cdot & h_{N r N t}
\end{array}\right)
$$

where $h_{n_{r} n_{t}}$ represents the channel transfer function of the wireless link between transmitter $n_{t}$ and receiver $n_{r}$.

The work by Foschini [62] provided a basis for approaches that employ multiple transmit antennas and multiple receive antennas in a system. When independent data streams are simultaneously transmitted from all the transmitters, the technique is called spatial multiplexing. In [62], Foschini defines an efficient technique for spatial multiplexing that breaks information data streams into substreams called diagonal Bell Laboratories layered space-time architecture (D-BLAST). D-BLAST employs antenna arrays at both the transmitter and receiver along with a diagonally-layered coding structure where code blocks are dispersed across diagonals in space-time. This leads to theoretical rates which grow directly proportional to the number of transmit and receive antennas in rich scattering environments. In fact, D-BLAST is shown to achieve up to $90 \%$ of the Shannon capacity [62].

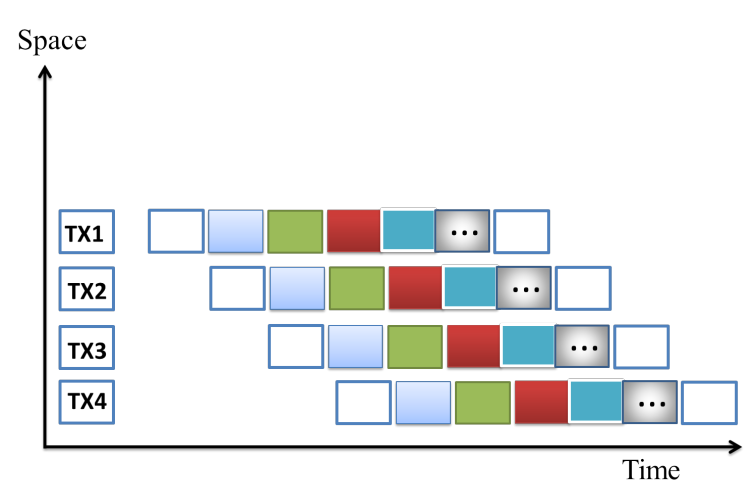

(a) D-BLAST

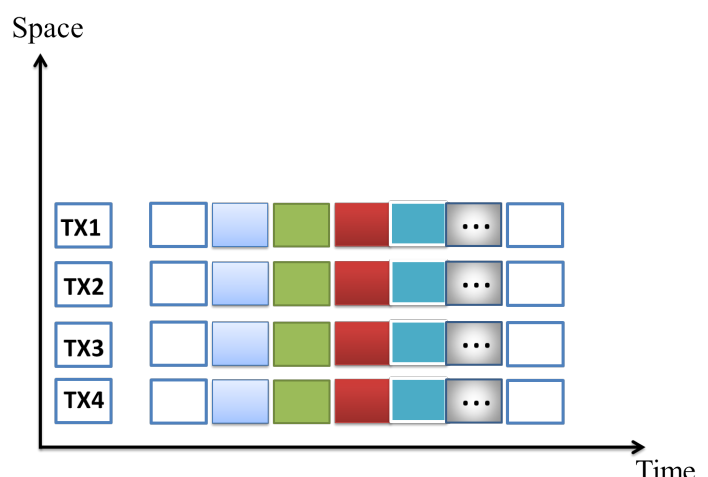

(b) V-BLAST

Figure 2.11: (a) D-BLAST and (b) V-BLAST coding for four antennas MIMO system

Another approach, called vertical Bell Laboratories layered space-time architecture (V-BLAST), was proposed in [67]. The fundamental difference between V-BLAST and D-BLAST is the vec- 
tor encoding process, where a vector is defined as the vector-valued transmitter (a combination of the transmit antenna substreams). On the one hand, in D-BLAST, vector encoding is introduced through the use of inter-substream block coding such that the block codes are organised along diagonals in space and time. In V-BLAST, the vector encoding is simply a demultiplexing operation of the original data stream, followed by independent bit to symbol mapping of each substream. In particular, no inter-substream coding is needed. The substream coding structure in D-BLAST and V-BLAST is illustrated in Figure 2.11 (a) and 2.11 (b) respectively, where the substreams from the same stream source are denoted by the same colour. Despite the fact that D-BLAST enables higher spectral efficiency, its deployment is hindered by its complexity. However both approaches are used in MIMO technique in the pursuit for higher data rates.

MIMO facilitates not only spatial multiplexing but also multiple transmit antennas may be used to increase the transmit diversity of a system. MIMO can also trade off both spatial multiplexing and transmit diversity simultaneously. The idea of using the multiple antennas for transmit diversity is known as space-time coding [65, 68,69]. A method that successfully achieved transmit-diversity for two transmit antennas was proposed and presented by Alamouti in [63]. Apart from the scheme presented by Alamouti, all other systems usually trade-off spatial multiplexing with transmit diversity [32,69]. Due to the potential of MIMO, these techniques are already widely used in several RF communication systems. For instance, multiple transmitter-receiver techniques are exploited in WLAN standards such as Institute of Electrical and Electronics Engineers (IEEE) 802.11n and provided data rates of $100 \mathrm{Mbit} / \mathrm{s}$ and higher [40]. Also the techniques are used in third-generation (3G) and fourth-generation (4G) wireless communication systems [70-75].

\subsection{Chapter summary}

In this chapter, the background and evolution of wireless communication systems was introduced and their applications in daily life was discussed. The chapter has also given a brief review of optical wireless transmission techniques for indoor applications. Optical wireless channel and propagation properties ware also reviewed. The eye safety issue for OWC where precautions and adherence to safety regulations was discussed. The fundamentals of OWC communication setup have been discussed, the geometry of the wireless link and their optical wireless channel (LOS and NLOS channel models) are presented. The typical building blocks of the transmitter and the receiver have been presented. 
The basic concepts and models for OWC that will be applied within the main chapters of this thesis have been introduced. Trade-offs within optical modulation techniques which employ IM and DD have been presented and discussed. Modulation techniques for single carrier and multicarrier transmission were reviewed and discussed. Compared to single carrier transmission, multi-carrier techniques such as OFDM, mitigate ISI by long symbol durations. However, the non-negativity constraint of the optical carrier affects these techniques. For instance, in DCOOFDM a high constant DC bias is required to make the bipolar OFDM signal non-negative. ACO-OFDM operates without a DC bias but at the expense of providing only half of spectral efficiency when compared with DCO-OFDM. For short symbol durations the ISI effect can be significant in single carrier systems.

In the chapter, the development and current deployment of MIMO systems was reviewed. MIMO techniques is known to provide high spectral efficiencies and therefore the techniques enable high data rates including for indoor OWC. However, indoor MIMO OWC links are highly correlated, as a result, indoor scenarios become more challenging for MIMO techniques which need uncorrelated links for better performance. This will be studied in detail in later chapters. 


\section{Chapter 3 Application of Single Carrier (SC) and Optical OFDM, in Multi-Cell Multi-User VLC System}

This chapter studies different scenarios in an indoor VLC system. The VLC Multi-Cell Multiuser system for capacity sharing in different locations of the room is designed and simulated so as to reduce the co-channel interference experienced by users and hence improve the system capacity. A DC biasing optimization algorithm for DCO-OFDM (DCO-Optimized) is also proposed and simulated for improvement of DCO-OFDM system spectral efficiency. Further, a statistical performance comparison for optical OFDM and Single carrier (SC) schemes operating over many room locations is provided.

\subsection{Introduction}

The basic system types of VLC fall into diffuse and line of sight (LOS) systems [76]. High data rates up to Gbits/s can be achieved in LOS conditions [1,13], but the systems are vulnerable to obstacles or shadowing because of their directionality. In diffuse VLC systems several paths exist from the source to receiver which makes the system robust to obstacle or shadowing $[10,11]$. However the path losses are high and multipath creates interference which limits the achievable data rate $[9,17]$. A promising solution to combat multipath distortion and increase data rate without any bandwidth or power expansion is to use OFDM techniques [54,77-80].

A tutorial overview of OFDM highlighting the aspects that are important in optical applications was given in [59]. It was suggested that to achieve good performance in optical systems, OFDM must be adapted in various ways. Impairments in optical systems which are caused by OFDM including high peak to average power ratio (PAPR) and its sensitivity to phase noise and frequency offset was described. Three optical OFDM techniques reported in the literature are asymmetrically clipped optical orthogonal frequency division multiplexing (ACO-OFDM) a direct current biased optical OFDM (DCO-OFDM) $[9,59,76,78,81]$ and unipolar OFDM 
(U-OFDM) [82, 83]. In ACO the bipolar OFDM signal is made unipolar by clipping the signal at zero before transmission $[59,84,85]$. In DCO, the bipolar OFDM generated by the transmitter is converted to a unipolar signal by adding direct current (DC) bias $[59,86-88]$ and in U-OFDM, a real bipolar OFDM signal obtained from OFDM modulator is transformed into a unipolar signal by encoding each time sample into a pair of new time samples $[82,83]$.

In [9], theoretical and simulation results for the performance of asymmetrically-clipped optical OFDM (ACO-OFDM) and DC-biased optical OFDM (DCO-OFDM) in AWGN for intensitymodulated direct-detection systems was presented. The results suggested that, for DCO-OFDM, the optimum bias depends on the constellation size which limits its performance in adaptive systems. ACO-OFDM requires less optical power for a given data rate than DCO-OFDM for all but the largest constellations and is better suited to adaptive systems as the same structure is optimum for all constellations. The comparison of ACO-OFDM and other modulation in AWGN was presented in [77], it was shown that ACO-OFDM with 4-QAM subcarrier modulation has the same bandwidth efficiency but requires $2 \mathrm{~dB}$ less energy per bit than on-off keying. ACOOFDM with larger constellation sizes gives higher bandwidth efficiencies and lower optical power than other modulation schemes.

The impact of double-sided signal clipping on optical wireless communication (OWC) systems employing (ACO-OFDM) was investigated in [85]. Analytical expressions for the attenuation factor and the clipping noise variance were determined and employed in the derivation of the electrical signal-to-noise ratio (SNR) at the receiver. The SNR showed that unlike in radio frequency based OFDM-based systems an SNR increase is not achievable simply by increasing the average electrical and/or optical power at the transmitter. In OWC, such a measure leads to a larger clipping distortion. Also higher order modulation proves to be more vulnerable to signal clipping, whereas the IFFT/FFT size does not significantly affect the BER performance. In [76] the performance of indoor orthogonal frequency division multiplexing (OFDM) optical wireless communication systems in the presence of light emitting diode (LED) nonlinear distortions was analysed. It was shown that LED clipping has a significant impact on the performance of both systems and an optimum system design should take into account the OFDM signal power, DC-bias point, and LED dynamic range. In [78], the electrical power requirement and the spectral efficiency of optical OFDM (DCO-OFDM and ACO-OFDM) transmission schemes for OWC were compared in the presence of front-end-induced double-sided signal clipping. It was shown that in a practical front-end biasing setup DCO-OFDM has a lower electrical power 
requirement to achieve a target BER as compared to ACO-OFDM for modulation orders with spectral efficiencies above $1 \mathrm{bit} / \mathrm{s} / \mathrm{Hz}$. In [79], the Performance Analysis of indoor OWC OFDM systems in the presence of LED clipping distortions is analysed. The performance of these systems, in terms of average electrical OFDM signal power versus bit-error-ratio (BER), DC power consumption, and transmitted optical power in the presence of additive white Gaussian noise (AWGN) channel and considering a practical LED model, were studied. The results highlighted that LED clipping has significant impact on the performance of these systems and that the OFDM signal power, DC bias point, and LED dynamic range need to be optimized.

In [81], the impact of clipping noise on optical wireless communication (OWC) systems employing OFDM was investigated. The BER performance of ACO-OFDM with DCO-OFDM is compared for different clipping levels and multi-level quadrature amplitude modulation schemes. It was found that ACO-OFDM is more robust to clipping effects than DCO-OFDM for similar modulation schemes at the expense of a $50 \%$ reduction in spectral efficiency. Therefore, ACOOFDM is more suitable for applications with lower radiated average optical power, whereas DCO-OFDM promises to deliver higher throughput.

A novel modulation technique called unipolar OFDM (U-OFDM) is proposed in [82].

U-OFDM uses different time sample states and an innovative rearrangement of the OFDM frame which allow for the creation of unipolar OFDM signals required for OWC with LEDs. In comparison to similar techniques like DC-biased optical OFDM (DCO-OFDM and ACOOFDM), U-OFDM is both optically and electrically more power efficient in an AWGN channel, which is prevalent in an optical wireless system. The issue of pulse shaping in U-OFDM systems was discussed in [83]. It was shown that, if pulse shaping is applied before clipping, bipolar pulse-shaping filters can be used for the three modulation schemes ACO-OFDM, UOFDM, and PAM-DMT, and in-band interference can be avoided. In [86] an optimized DCOOFDM technique capable of transmitting transmit large constellations with a moderate DC bias was proposed. To reduce the clipping impact, a linear companding function was used in order to compress the negative part of the bipolar signal and therefore to reduce the amount of the clipped peaks and the loss of information. It was demonstrated that the proposed technique has better bit error rate and optical power performance than conventional DCO-OFDM and ACO-OFDM.

The application of these optical OFDM techniques in different indoor VLC scenarios is analysed in this chapter. An indoor optical wireless scenario where there are a number of light 
sources and many users is considered. As the optical links have very limited transmission range caused by noise from ambient light and high path loss, an indoor optical multi-user is established so that light beam from LEDs focus on a specific area to save different mobile devices with low interference. The performance of optical OFDM and single carrier (SC) techniques are then compared for single user and multi-user systems.

\subsection{System descriptions}

In this section the basic optical communication system including the transmitter-receiver geometry and channel model will be described. Also its system model will be discussed and signal to noise ratio (SNR) will be evaluated.

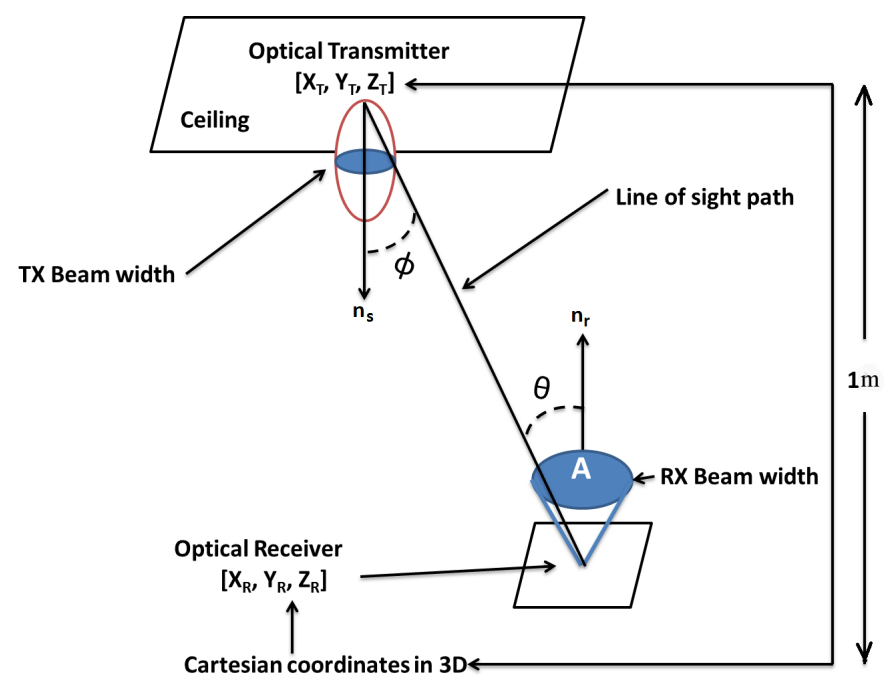

Figure 3.1: Transmitter and receiver geometry in basic VLC system

\subsubsection{Basic optical wireless communication system}

The basic communication system comprises one transmitter fixed on the ceiling of the room and is directed downwards perpendicular to the floor. the receiver is located at human waist height pointing upward towards the direction of transmitter. Consider an indoor line of sight (LOS) optical system propagation path as shown in Figure 3.1, the channel gain from the transmitter 
(LED) to the receiver (PD) is given as follows: [76]

$$
h=\left\{\begin{array}{cc}
\frac{A(\kappa+1) \cos ^{\kappa}(\phi) \cos (\theta)}{2 \pi d^{2}}, & 0 \leq \theta \leq \varphi_{\frac{1}{2}} \\
0, & \theta>\varphi_{\frac{1}{2}}
\end{array}\right.
$$

where $\kappa=\frac{-\ln (2)}{\ln \left(\cos \left(\Phi_{\frac{1}{2}}\right)\right)}$. The scalar $A$ is the collection area of the detector, $\Phi_{\frac{1}{2}}$ is the transmitter semi-angle (at half power) and the scalar $\varphi_{\frac{1}{2}}$ is the field of view (FOV) semi angle of the receiver. The angle $\phi$ denotes the transmitter viewing angle with respect to the receiving unit, $\theta$ denotes the angle of incidence on the photodiode (angle with respect to the vector linking the transmitter and receiver). The channel gain $h$ depends on the specific position of the transmitter and the receiver. The scalar $d$ represents the distance between transmitting unit and receiver. Vectors $\mathbf{n}_{\mathbf{s}}$ and $\mathbf{n}_{\mathbf{r}}$ in Figure 3.1 are vectors representing the transmitter and receiver axis respectively. If a receiver and a transmitter are not in each other's FOV, $h=0$ holds.

A number of scenarios are considered as follows. The first one is a single user-single beam light; this was considered to determine the system coverage and channel capacity for one wide beam light with only one user. The second one is a single user-multibeam light; this was chosen to determine whether changing the power distribution from one LED cell to 4 LED cells would increase coverage and capacity of the system. Finally, the third model of a multiuser-multibeam scenario was suggested to determine the advantage of sharing communication among multiple users in multiple cells in the room.

\subsubsection{System model}

Consider Figure 3.1, the received line of sight (LOS) signal vector at any location of the transmitter as in [76] is given by:

$$
y_{1}=h_{11} x_{1}+n
$$

where $x_{1}$ is the transmitted symbol and $y_{1}$ is the received signal, $h_{11}$ is the radiation channel gain of the transmitter to one user and $n$ represents noise which is assumed to be real valued additive white Gaussian noise (AWGN) with zero mean and double sided spectral density $\delta^{2}$. The optical power received at the receiver can be calculated as in $[8,89,90]$,

$$
P_{r}=P_{t} h_{11}
$$


where, $P_{r}$ and $P_{t}$ are the transmitted and received powers respectively. A photodiode is used to convert the received optical power into an electrical current and the output is given by:

$$
\varpi=P_{r} \varrho
$$

where, $\varrho$ is the photodiode responsivity and the signal to noise ratio (SNR) is given by:

$$
\mathrm{SNR}=\frac{\left(P_{r} \varrho\right)^{2}}{\delta^{2}}
$$

where, $\delta^{2}$ is a total noise variance calculation as in $[18,89,91,92]$, and is given by:

$$
\delta^{2}=\delta_{s s}^{2}+\delta_{b g}^{2}+\delta_{d c}^{2}+\delta_{j s}^{2}
$$

where $\delta_{s s}^{2}$ is the photon-generated noise or shot-noise in the detector that results from the received signal and is given by:

$$
\delta_{s s}^{2}=2 q \varrho P_{r} B
$$

where, $q$ is the electron charge and B is the electron bandwidth. The scalar $\delta_{b g}^{2}$ is the variance caused by background electronic noise power and is given by:

$$
\delta_{b g}^{2}=2 q \varrho P_{b g} B
$$

where, $P_{b g}$ is background radiated power. $\delta_{d c}^{2}$ is caused by the dark current of the photodiode and is given by:

$$
\delta_{d c}^{2}=2 q \varrho I_{d c} B
$$

where, $I_{d c}$ is the dark current in the detector. $\delta_{j s}^{2}$ is the variance in the detector that results from Johnson (Thermal) noise and is given by:

$$
\delta_{j s}^{2}=\frac{4 K T_{e} B F}{R_{l}}
$$

where, $F$ is the noise figure of the receiver optical amplifier [93], $T_{e}$ is the equivalent temperature, $K$ is the Boltzmann constant and $R_{l}$ is the load resistance. The parameters for modelling Johnson noise are discussed in section 3.4. The total noise variance calculation in equation (3.6) shows that, the shot and thermal noises are dominant. Therefore, in the next chapters other parts of the equation will be ignored. 


\subsection{System under consideration}

The system under consideration is based on different scenario simulations using OFDM and (SC). In an optical wireless systems, the OFDM signal must be represented as intensity, i.e. the modulating signal must be both real and positive whereas baseband OFDM signal are generally complex and bipolar. A real baseband signal can be generated by constraining the OFDM frequency domain symbol vector to have Hermitian symmetry $[9,76]$. For this work in particular, the data at the receiver are retrieved using adaptive modulation techniques and the computed SNR at the receiver is fed back periodically to the transmitter and is used to choose the appropriate modulation level.

\subsubsection{Asymmetrically clipped optical OFDM (ACO-OFDM)}

In ACO-OFDM only odd subcarriers are modulated and even subcarriers as set to zero to ensure that the output consists of only real values [9]. Figure 3.2 (a) shows the block diagram of IM/DD system using ACO-OFDM modulation. In this system, the input serial data is partitioned into $N$ parallel data streams and mapped onto frequency samples using quadrature amplitude modulation (QAM) or pulse amplitude modulation (PAM). To ensure real valued OFDM signals, the input frequency symbols to the IFFT block are constrained to have Hermitian symmetry and follow the data format shown in Figure 3.2 (b). At the digital analog converter (D/A) output, the time continuous signal $x(t)$, is made positive by clipping the negative amplitudes. The resulting unipolar signal is then modulated onto the power intensity of the optical transmitter (Laser diode LD or a light emitting diode LED). At the receiver, a photodiode detects the optical signal and delivers an electric current proportional to the detected optical power. The discrete time signal at the analog digital converter (A/D) output is converted back to a parallel signal and input to a fast Fourier transform (FFT) block in order to recover the transmitted frequency symbols. The bit stream is finally demodulated to recover the original transmitted data. For the system with subcarriers 1 to $N$, the data format is

$$
\mathbf{s}=\left[\begin{array}{lllllllllllllll}
0 & s_{0} & 0 & s_{1} & 0 & s_{2} \ldots . . s_{N / 4-1} & 0 & s_{N / 4-1}^{*} & 0 & s_{2}^{*} & 0 & s_{1}^{*} & 0 & s_{0}^{*}
\end{array}\right]
$$

where, $s_{c}$ denotes the data transmitted on the odd subcarrier index $1,3,5 \ldots N-1$, and [.]* denotes complex conjugate. The OFDM modulator produces a halfwave symmetric real valued signal. That means the same information in the first half of the samples is repeated in the 


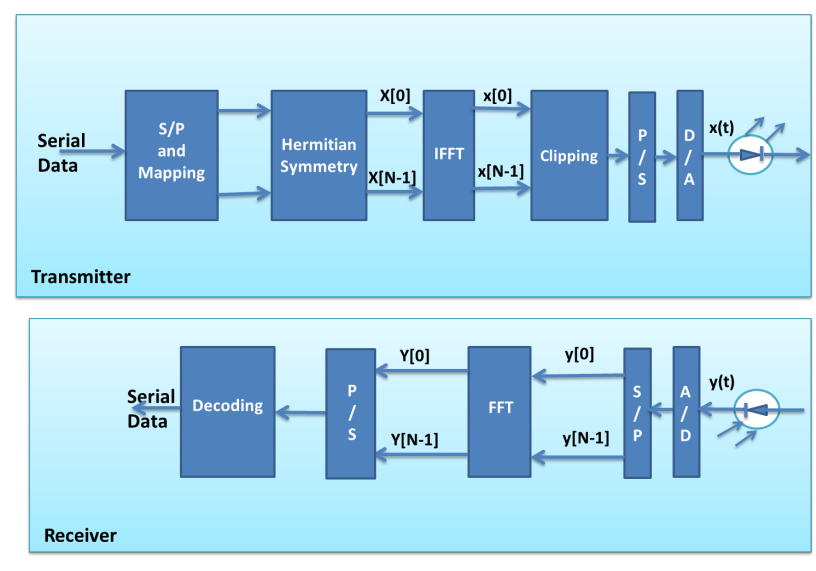

(a)

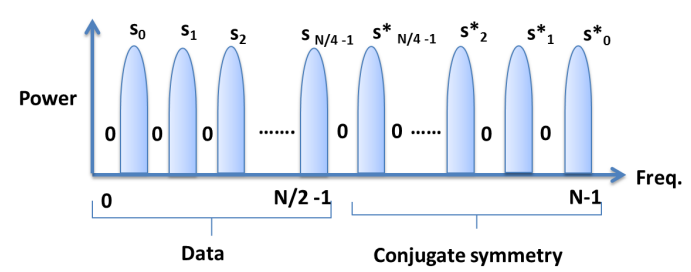

(b)

Figure 3.2: (a) Block diagram of ACO-OFDM system (b) Data format of ACO-OFDM

second half of the OFDM symbol. As a result the negative part can be clipped without any loss of information $[9,76]$. The modulation used is quadrature amplitude modulation (4 QAM to 1024 QAM) and the FFT size $(N)$ is 32 . Since only $1 / 4$ of the $N$ subcarriers contain distinct data symbols, the number of bits in one packet (one OFDM symbol) can be calculated by

$$
N_{b_{\{\mathrm{ACO}\}}}=\left(\frac{N}{4}\right) \log _{2} M \text { bits }
$$

where $M$ is the size of the QAM modulation. The packet error probability or the number of incorrectly received data packets divided by the total number of received packets $\left(\gamma_{\{\mathrm{ACO}\}}\right)$ can be calculated as in [18]

$$
\gamma_{\{\mathrm{ACO}\}}=1-\left(1-P_{e}\right)^{N_{b_{\{\mathrm{ACO}\}}}}
$$

where $P_{e}$ is the bit error probability (BER) which is the ratio of the number of error bits to the number of transmitted bits. A packet is declared incorrect if at least one bit is erroneous. The achieved data rate for the ACO-OFDM system as in [76, 87], can be given by:

$$
R_{\{\mathrm{ACO}\}}=B\left(\frac{N_{b_{\{\mathrm{ACO}\}}}}{N}\right) \mathrm{bps}
$$

where $B$ is the system bandwidth. Therefore the normalised (devided by the system bandwidth $B$ ) throughput for ACO including packet errors is given by:

$$
T h_{\{\mathrm{ACO}\}}=R_{\{\mathrm{ACO}\}}\left(1-\gamma_{\{\mathrm{ACO}\}}\right) \mathrm{bps} / \mathrm{Hz}
$$


The flowchat (Figure 3.3) illustrates how throughput is calculated. The chart shows step by step from data modulation and mapping, channel gain calculations, SNR calculations, decision of appropriate $M$-QAM, BER calculations and then output throughput.

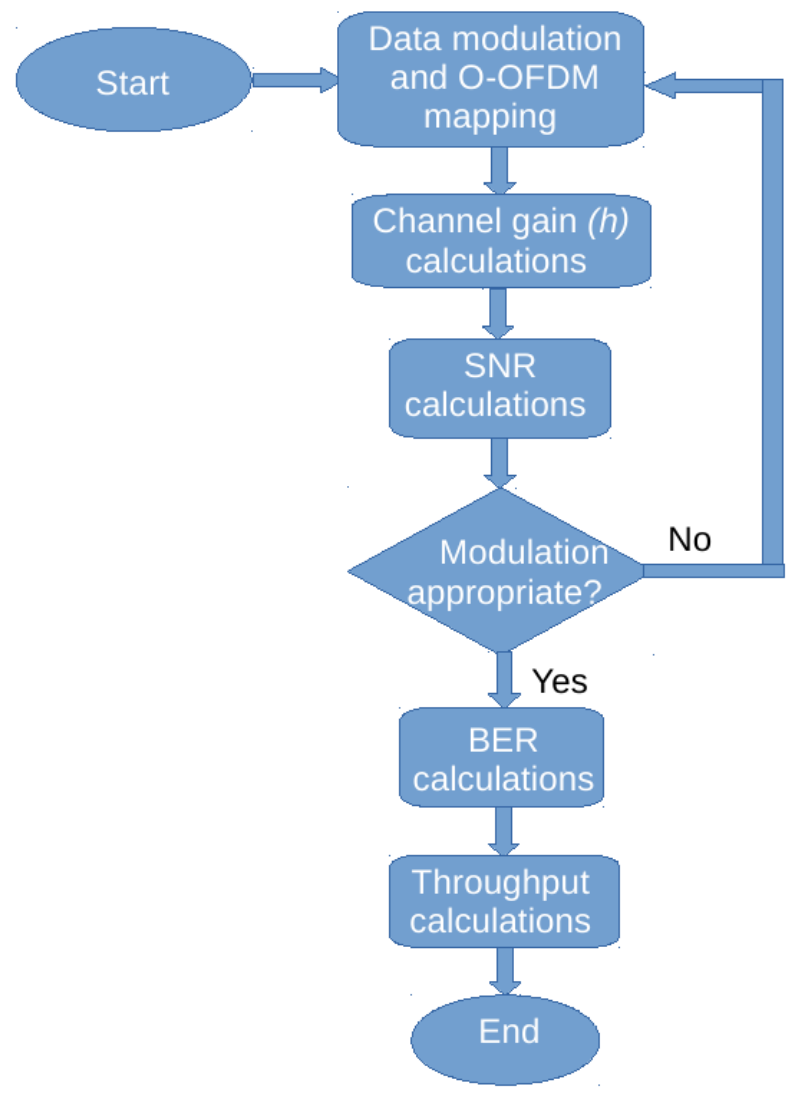

Figure 3.3: Flow chart to illustrate throughput calculations

\subsubsection{Direct current biased optical OFDM (DCO-OFDM)}

In DCO-OFDM, data are assigned to all odd and even subcarriers except the first and $(N / 2+1)$ subcarriers which must be set to zero to ensure that the output consists only of real values [76]. Figure 3.4 (a) shows the block diagram of IM/DD system using DCO-OFDM modulation. To ensure real valued OFDM signals, the input frequency symbols to the IFFT block are constrained to have Hermitian symmetry and follow the data format shown in Figure 3.4 (b). To ensure a unipolar signal transmission, a DC bias is added to the bipolar signal and then removed at the receiver. 


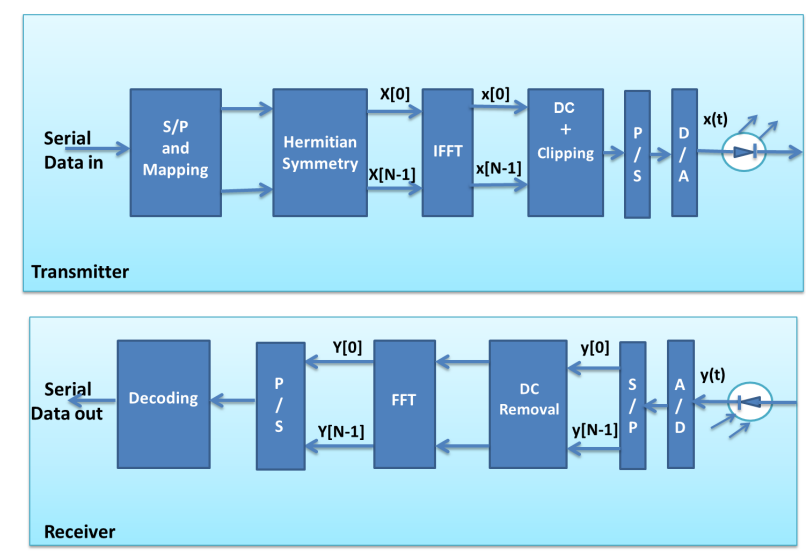

(a)

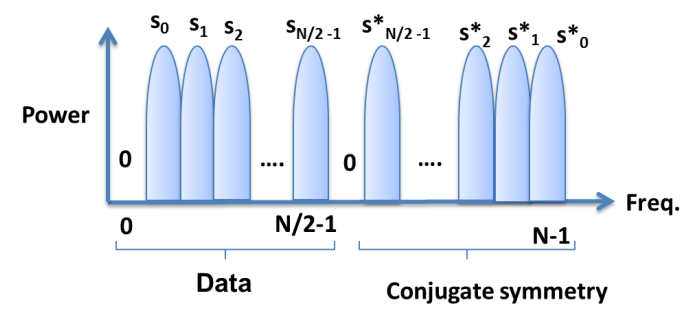

(b)

Figure 3.4: (a) Block diagram of DCO-OFDM system (b) Data format of DCO-OFDM

For the system with subcarriers 1 to $N$, the data format is

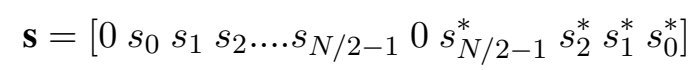

The bipolar $x(t)$ OFDM signal is then converted to a unipolar signal by adding a DC bias $\left(B_{D C}\right)$ as shown in (3.17).

$$
x(t)=x_{o}(t)+B_{D C}
$$

where, $x(t)$ is the unipolar signal obtained after adding the $D C$ bias and $x_{o}(t)$ is the bipolar signal.

The DCO-OFDM biasing DC voltage is calculated based on the following equation

$$
B_{D C}=\xi \sqrt{\mathrm{E}\left\{x_{o}^{2}(t)\right\}}
$$

$B_{D C}$ is defined as a bias of $10 \log _{10}\left(\xi^{2}+1\right) \mathrm{dB}$ [77], where $\xi^{2}$ is the clipping factor. The modulation used is QAM (4-QAM to 1024-QAM) and the FFT size $(N)$ is 32 . Since only $(N / 2-1)$ of $N$ subcarriers contain distinct data symbol, the number of bits in one packet (one OFDM symbol) can be calculated by

$$
N_{b_{\{\mathrm{DCO}\}}}=\left(\frac{N}{2}-1\right) \log _{2} M \text { bits }
$$


The packet error probability $\left(\gamma_{\{\mathrm{DCO}\}}\right)$ can be calculated as

$$
\gamma_{\{\mathrm{DCO}\}}=1-\left(1-P_{e}\right)^{N_{b_{\{\mathrm{DCO}\}}}}
$$

The achieved data rate for DCO-OFDM system, is given by:

$$
R_{\{\mathrm{DCO}\}}=\left(\frac{N_{b_{\{\mathrm{DCO}\}}}}{N}\right) \text { bps }
$$

Therefore the normalised throughput for DCO is given by:

$$
T h_{\{\mathrm{DCO}\}}=R_{\{\mathrm{DCO}\}}\left(1-\gamma_{\{\mathrm{DCO}\}}\right) \mathrm{bps} / \mathrm{Hz}
$$

Two DC bias methods are used, the first one is DCO-13dB where DC bias voltage level of $13 \mathrm{~dB}$ is used [9] (see figure 3.5). The second one is a new proposed DCO method called DCO-Optimized. 


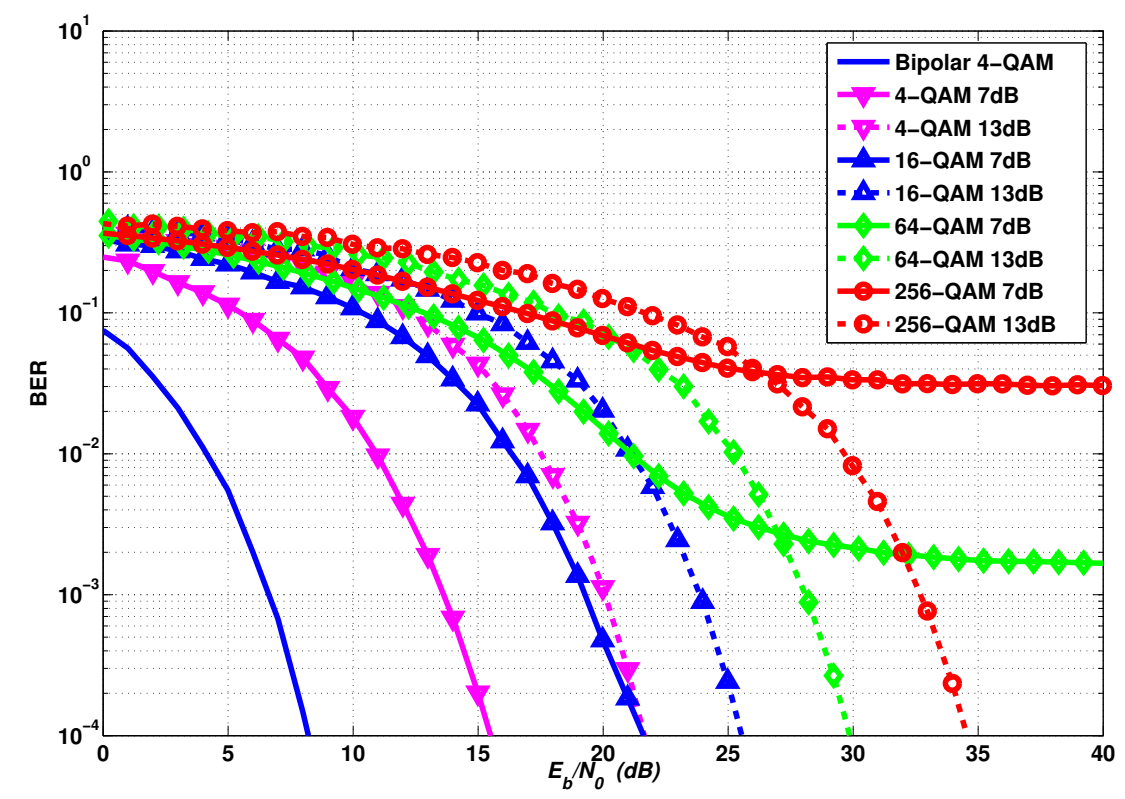

(a)

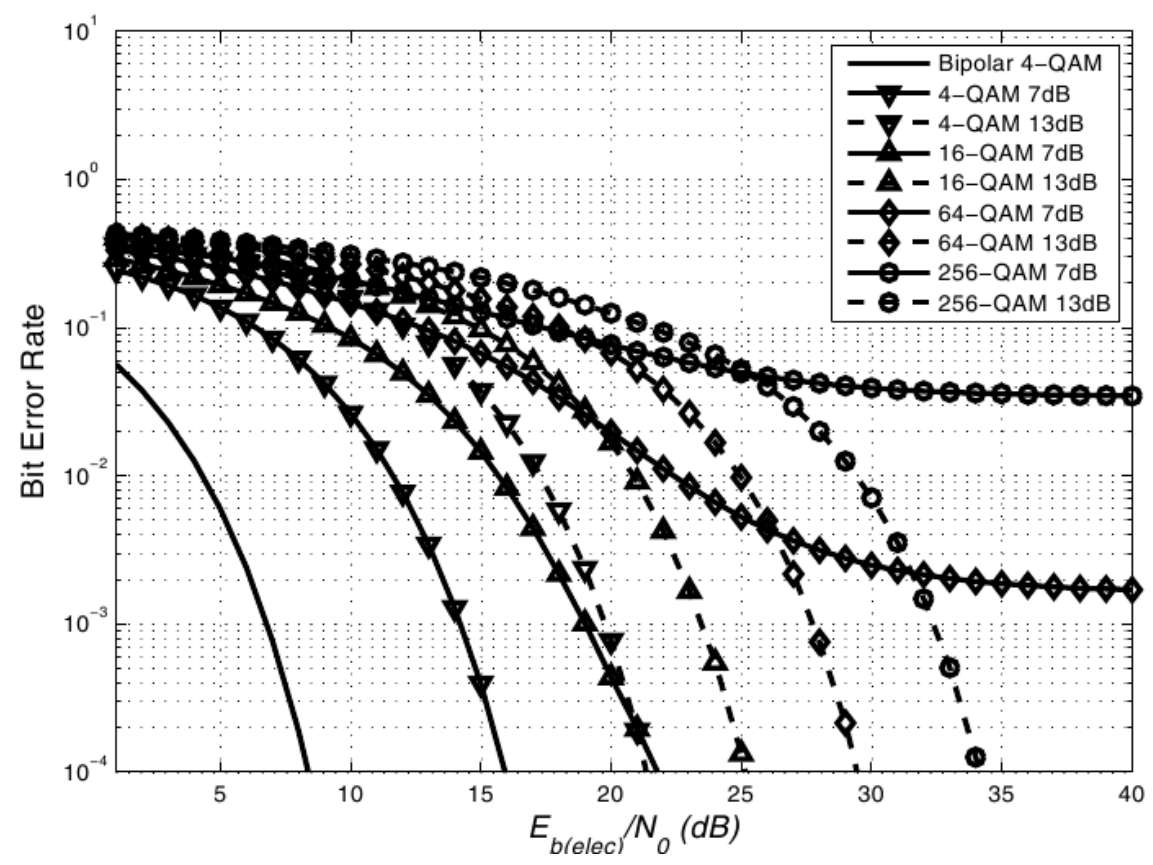

(b)

Figure 3.5: $B E R$ in $A W G N$ versus $E_{b} / N_{0}$ for DCO-OFDM with DC bias of $7 d B$ and $13 d B$ using 4 QAM, 16 QAM, 64 QAM and 256 QAM (a) Simulated to compare with Figure 2 of [9] (b) Copy of Figure 2 of [9] 


\subsubsection{DCO-Optimized}

In DCO-Optimized, the three DC voltage levels are used, i.e. $7 \mathrm{~dB}$ for 4-QAM, 8-QAM, and 16-QAM then, $10 \mathrm{~dB}$ for 32-QAM, and 64-QAM while $13 \mathrm{~dB}$ is applied in 128-QAM, 256QAM, 512-QAM and 1024-QAM. The DCO-Optimized is proposed because the lower QAM modulation level can achieve low bit error probabilities with a lower DC bias, which cannot be achieved by high order modulation schemes at the same bias level [9]. For this method, equation (3.17) becomes

$$
x(t)=x_{o}(t)+\left\{\begin{array}{c}
7 \mathrm{~dB}, 4 \mathrm{QAM} \leq M \leq 16 \mathrm{QAM} \\
10 \mathrm{~dB}, 16 \mathrm{QAM}<M \leq 64 \mathrm{QAM} \\
13 \mathrm{~dB}, 64 \mathrm{QAM}<M
\end{array}\right.
$$

\subsubsection{Single Carrier-Pulse Amplitude Modulation (PAM)}

Figure 3.6 (a) shows the block diagram of IM/DD using PAM and Figure 3.6 (b) shows the data format for a single carrier system. To ensure non-negativity in Pulse Amplitude Modulated symbols, all constellation points must be greater than or equal to zero. For fair comparison the packet length for SC was also set to the length of OFDM symbol for ACO and DCO i.e $N$ symbols. The modulation used was pulse amplitude modulation (2-PAM to 1024-PAM) and the packet size $(N)$ was 32 .

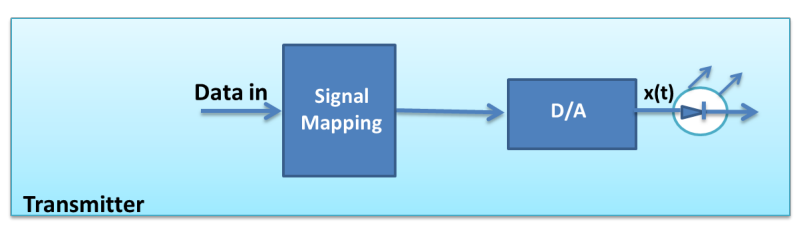

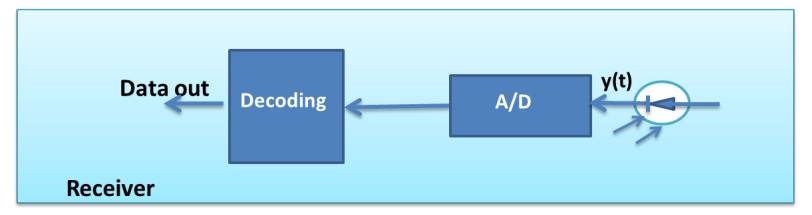

(a)

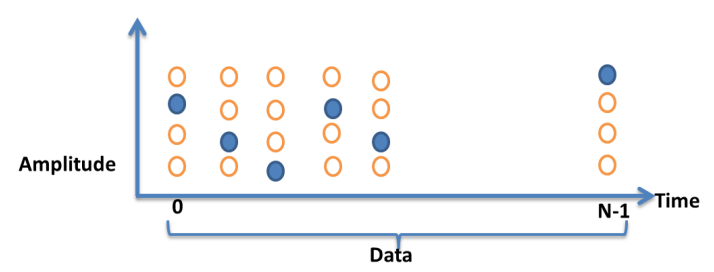

(b)

Figure 3.6: (a) Block diagram of SC system using PAM (b) Data format of SC system 
For one packet the number of bits can be calculated by

$$
N_{b_{\{\mathrm{PAM}\}}}=N \log _{2} M \text { bits }
$$

The Packet error probability $\left(\gamma_{\{\mathrm{PAM}\}}\right)$ can be calculated as

$$
\gamma_{\{\mathrm{PAM}\}}=1-\left(1-P_{e}\right)^{N_{b_{\{\mathrm{PAM}\}}}}
$$

The achieved data rate for Single carrier (SC) system, is given by:

$$
R_{\{\mathrm{PAM}\}}=\left(\frac{N_{b_{\{\mathrm{PAM}\}}}}{N}\right) \mathrm{bps}
$$

Therefore the throughput for SC is given by:

$$
T h_{\{\mathrm{PAM}\}}=R_{\{\mathrm{PAM}\}}\left(1-\gamma_{\{\mathrm{PAM}\}}\right) \mathrm{bps} / \mathrm{Hz}
$$

\subsubsection{Simulation scenarios}

The simulation scenarios are based on a single LED cell and four LED cells where single and multiple users are considered. These are shown in Figure 3.7 below

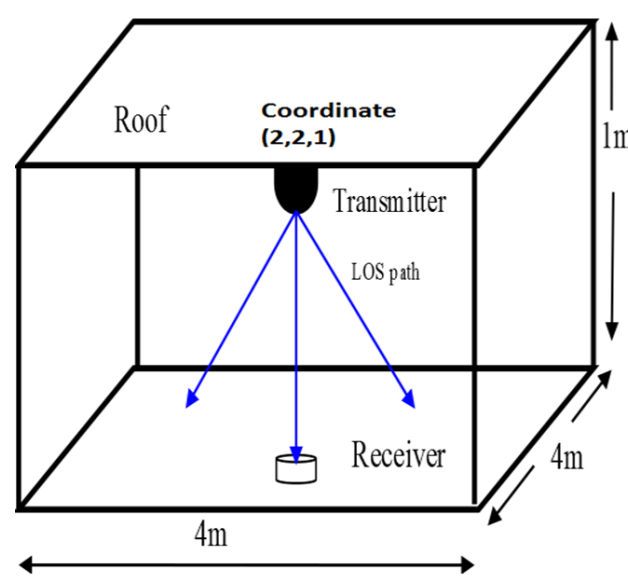

(a)

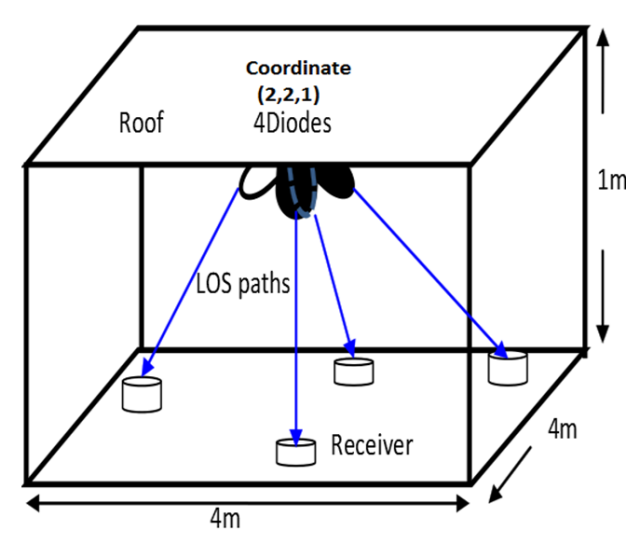

(b)

Figure 3.7: (a) Single beam light (b) Multibeam lights 


\subsubsection{Single user single beam light}

Consider Figure 3.7(a): this scenario aims at finding the data throughput distribution in the different room locations when the LED is pointing straight downwards. In this case the field of view (FOV) of the transmitter and receiver are $180^{\circ}$ which represents a theoretical upper bound on practical values. The system model for this scenario is based on equation (3.2) and its signal to noise ratio (SNR) at the receiver is computed using equation (3.5).

\subsubsection{Single user multibeam light}

Consider Figure 3.7(b) with only one user. In this scenario user locations are uniformly distributed throughout the room. The throughput distribution in the different room locations is calculated when using four LEDs pointing to different angles of the room, at an elevation angle of $45^{\circ}$ each and separated from each other by an azimuth angle of $90^{\circ}$. In this case the FOV of the transmitter and receiver are $90^{\circ}$. The received signal vector for user 1 in any part of the room is given by:

$$
y_{1}=h_{1 j} x_{j}+\sum_{i=1, i \neq j}^{4} h_{1 i} x_{i}+n
$$

where, the first term of equation (3.28) is the LOS signal for data transmission while the second term represents interference from the other transmitting LEDs. In most of the locations the three interference terms in equation (3.28) will be equal to zero. At the receiver the signal to noise and interference ratio (SNIR) is given by:

$$
\mathrm{SNIR}=\frac{\left(P_{t} h_{1 j} \varrho\right)^{2}}{\sum_{i=1, i \neq j}^{4}\left(P_{t} h_{1 i} \varrho\right)^{2}+\delta^{2}}
$$

\subsubsection{Multiuser multibeam}

Figure 3.7(b) is considered in this scenario. Fou cells system with multiple users in the room is considered. The aim is to observe how much the system can increase the capacity when users in the room share the cells. The throughput distribution in the different room locations is calculated in each cell and summed up to get the total throughput for the 4 cell system. In this case the four LEDs and PDs are set as depicted in Figure 3.7(b). The average throughput in cell 
$j$ is given by:

$$
T_{h_{c e l l}}^{\{j\}}=\frac{1}{U} \sum_{i=1}^{U} t_{h_{i j}}
$$

Therefore the total throughput is given by:

$$
T_{h_{\text {full }}}=\frac{1}{U} \sum_{i=1}^{U} \sum_{j=1}^{4} t_{h_{i j}}
$$

where, $t_{h_{i j}}$ is the individual user throughput for user $i$ in cell $j$ and $U$ is the number of users. The scalar $t_{h_{i j}}$ is computed based on equations (3.15), (3.22), or (3.27) for ACO, DCO and SC respectively. Adaptive modulation is used to obtain the throughput at any user location, i.e. the throughput calculated using different modulation levels for ACO, DCO and SC were stored in a lookup table and the maximum throughput determined according to the signal to noise ratio computed at the receiver.

\subsection{Simulation results}

A computer program was written using MATLAB software that implements the scenarios presented in previous sections. Table 3.1 shows the parameters used for simulation.

\begin{tabular}{|c||c|}
\hline Parameters & Values \\
\hline Room size $(W \times L \times H)$ & $4 \mathrm{~m} \times 4 \mathrm{~m} \times 3 \mathrm{~m}$ \\
\hline Number of LEDs & 1,4 \\
\hline Vertical distance from ceiling to receiver plane & $1 \mathrm{~m}$ \\
\hline Average transmitted power (per LED) & $27 \mathrm{dBm}$ \\
\hline Transmitter/Receiver field of view $\Phi, \psi$ & $90^{\circ}, 180^{\circ}$ \\
\hline Photodiode responsivity $(\varrho)$-eqn $(3.4)$ & $0.5 \mathrm{~A} / \mathrm{W}$ \\
\hline FFT/IFFT length $(N)$ & 32 \\
\hline Electron bandwidth $(\mathrm{B})$-eqn $(3.7-3.10)$ & $100 \mathrm{MHz}$ \\
\hline Dark current $\left(I_{d c}\right)$ - eqn $(3.9)$ & $1 \mathrm{nA}$ \\
\hline Photodiode area $(A)$ & $1 \mathrm{~cm}{ }^{2}$ \\
\hline Back ground radiation power $\left(\delta_{b g}^{2}\right)$-eqn $(3.8)$ & $0.1 \mu \mathrm{W}$ \\
\hline Noise figure $($ F) $[93$ ] of the $\mathrm{RX}$ Amplifier-eqn $(3.10)$ & $5 \mathrm{~dB}$ \\
\hline Transmitter coordinate $(3 \mathrm{D})$ & {$[2,2,1]$} \\
\hline Equivalent temperature $\left(T_{e}\right)$-eqn $(3.10)$ & $300 \mathrm{~K}$ \\
\hline Load resistance $\left(\mathrm{R}_{l}\right)$-eqn $(3.10)$ & $10 \mathrm{~K} \Omega$ \\
\hline
\end{tabular}

Table 3.1: Parameters used for simulation 
For validation of simulation, the output OFDM time signals for the ACO-OFDM and DCOOFDM systems are simulated (Figure 3.8). Figure 3.8 (a) and (b) show the bipolar OFDM time signals for ACO-OFDM and DCO-OFDM respectively after the IFFT process. Figure 3.8 (c) shows the unipolar ACO-OFDM signal obtained after clipping the negative part of the signal while Figure 3.8 (d) shows the unipolar DCO-OFDM signal obtained after adding DC bias to bipolar signal. Also the BER for ACO-OFDM and DCO-OFDM are simulated as shown in Figure 3.9 and 3.5 which closely match Figure 1 and 2 of [9]. In Figure 3.5 (a) the two plateu curves show the clipping noise in large constellations when lower DC bias level is used.

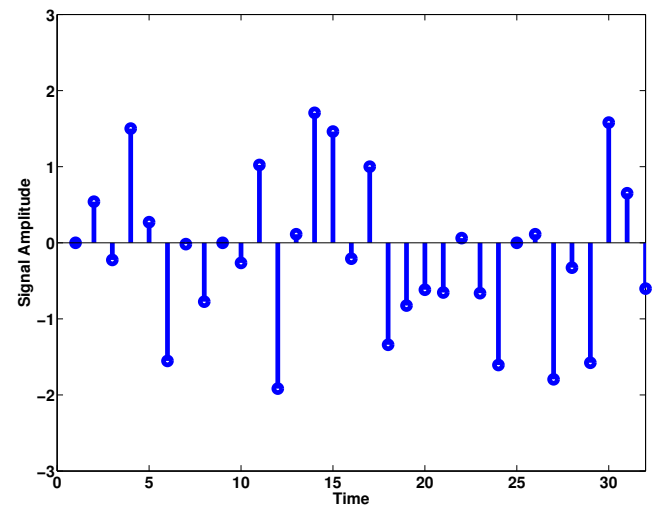

(a)

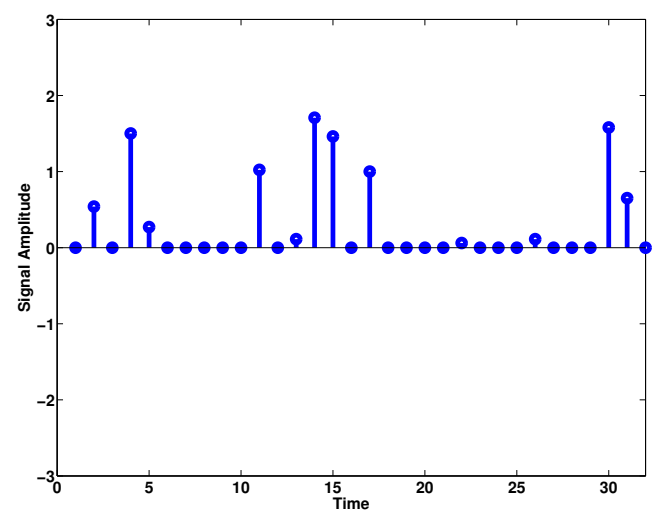

(c)

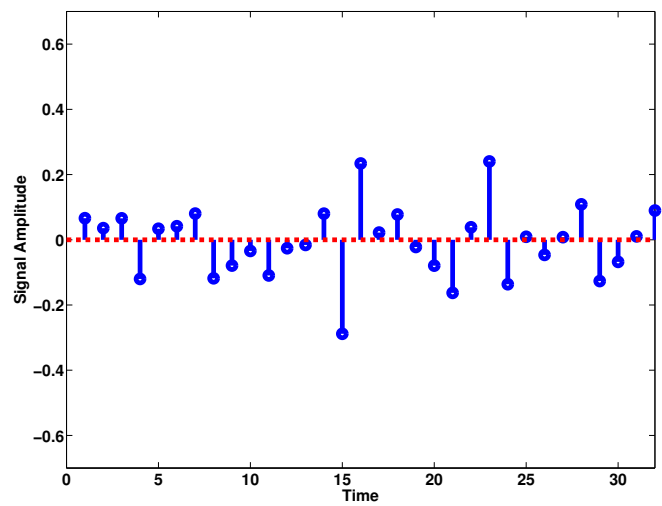

(b)

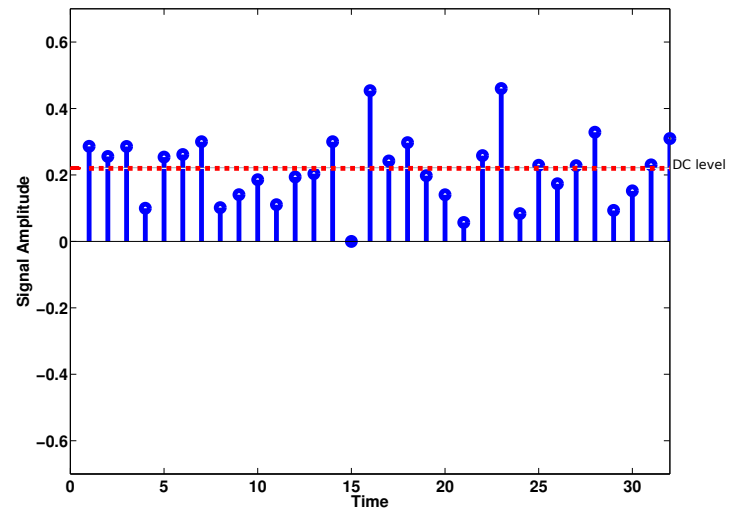

(d)

Figure 3.8: The output OFDM time signals for the ACO-OFDM and DCO-OFDM systems for $N=32$. (a) Bipolar ACO-OFDM (not clipped) (b) Bipolar DCO-OFDM (before DC biasing) (c) Unipolar ACO-OFDM (clipped) (d) Unipolar DCO-OFDM (after DC biasing) 


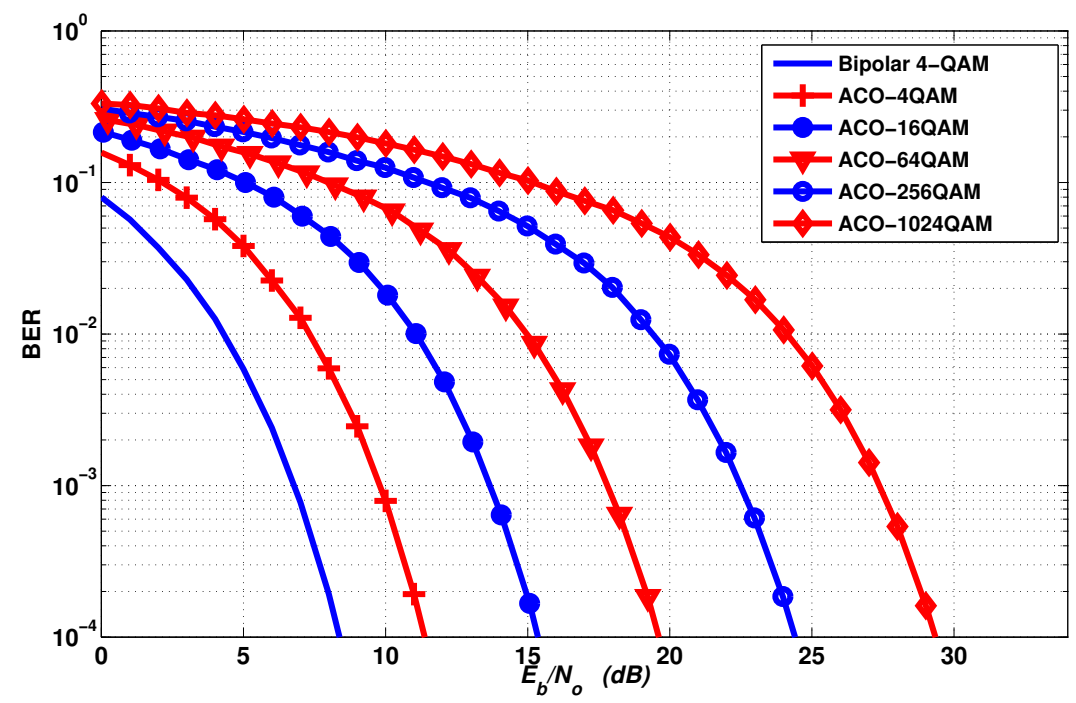

(a)

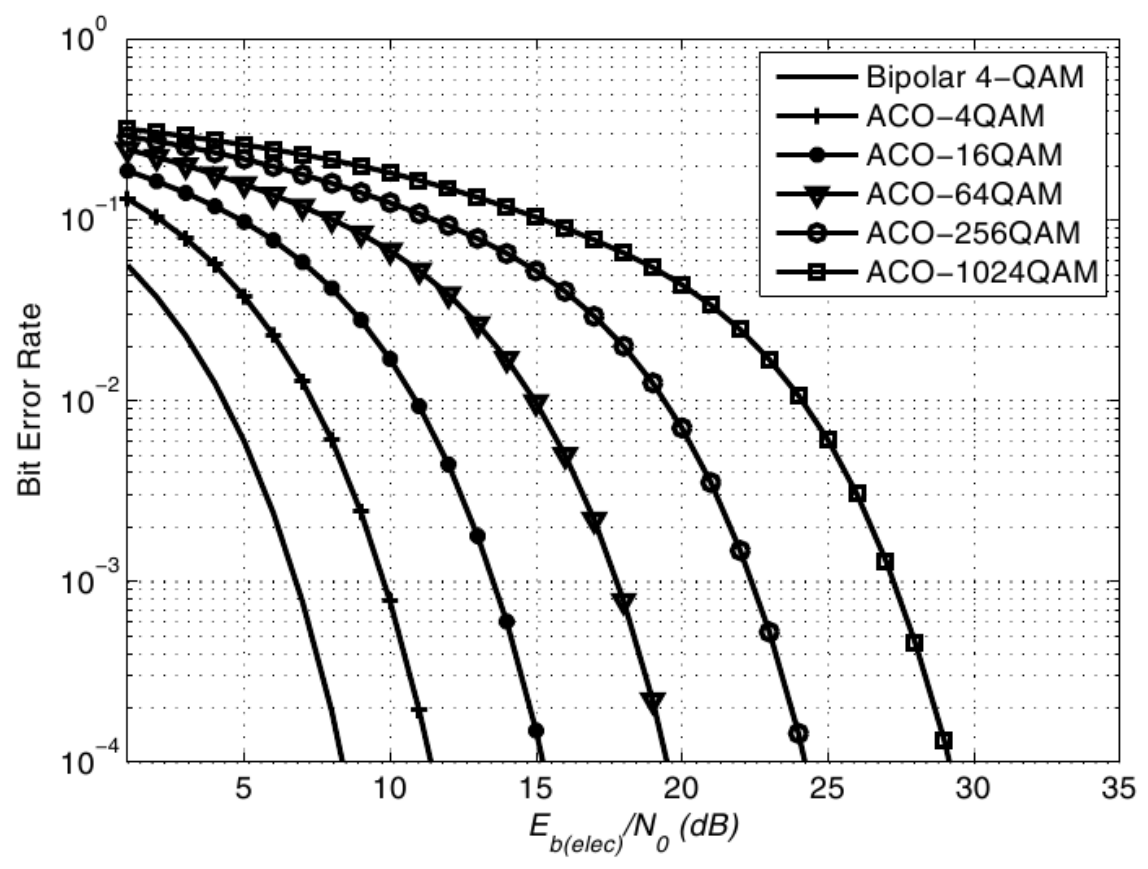

(b)

Figure 3.9: $B E R$ in $A W G N$ versus $E_{b} / N_{0}$ for bipolar $4 Q A M$ and ACO-OFDM using 4 QAM, 16 QAM, 64 QAM, 256 QAM, and 1024 QAM (a) Simulated to compare with Figure 1 of [9] (b) Copy of Figure 1 of [9] 


\subsubsection{Single user single beam light simulation}

Scenario one is simulated using the following parameters: the FOV of transmitter and receiver is $180^{\circ}$. The LED is pointing downward $90^{\circ}$ to the horizontal, as shown in Figure 3.7(a). The system performance is compared for ACO-OFDM, DCO-OFDM and SC-PAM. There are 10,000 uniformly distributed locations in the room that are used during this simulation. The results that are obtained as shown in Figure 3.10 and Figure 3.11.

Figure 3.10 shows the results of the CDF comparison between ACO, DCO-13dB, DCO-Optimized and PAM. The transmitter was pointing downward perpendicular to $\mathrm{x}, \mathrm{y}$ plane while the receiver was moving uniformly in the room with its normal pointing upward perpendicular to $\mathrm{x}, \mathrm{y}$ plane. Results for this scenario indicate that PAM provides a better performance interms of throughput compared to other schemes. Looking at the figure, PAM provides the throughput of about $2.5 \mathrm{bits} / \mathrm{s} / \mathrm{Hz}$ at $50 \%$ level while other schemes give the throughput lower than $2 \mathrm{bits} / \mathrm{s} / \mathrm{Hz}$ at the same level. At the lower modulation level (4-QAM to 64-QAM), ACO performs better in spectral efficiency than DCO with a fixed offset level and provides about the same throughput as optimized DCO. Also optimized DCO shows an improvement in performance compared to DCO with a fixed offset level. Figure 3.11 is a scatterplot of the room throughput distribution for scenario one, i.e. one light beam with one receiver. It is observed that for the room dimension used, the throughput is concentrated at the centre of the room. The red space in Figure 3.11 denotes the throughput lower than $10^{-4}$ bps which is assumed to be effectively zero throughput. With no considerations of reflections the regions with no coverage at the corners of the room is observed which indicate the effect of distance on channel gain. This drawback is addressed by the 4 cell system.

\subsubsection{Single user multibeam light simulation}

In this scenario .In this simulation, 4 cells and one receiver with either one, or four detector(s) were considered. The scenario provides better coverage by distributing the same power to the lower FOV LEDs. The setup and simulation parameters follow subsection 3.3.4.2 and the RX was moved as in subsection 3.4.1. For the case of the receiver the FOV was $180^{\circ}$ when only one detector was used and $90^{\circ}$ when four detectors were used. For more than one detector, the SNR obtained from each detector was compared and the highest SNR was chosen. 


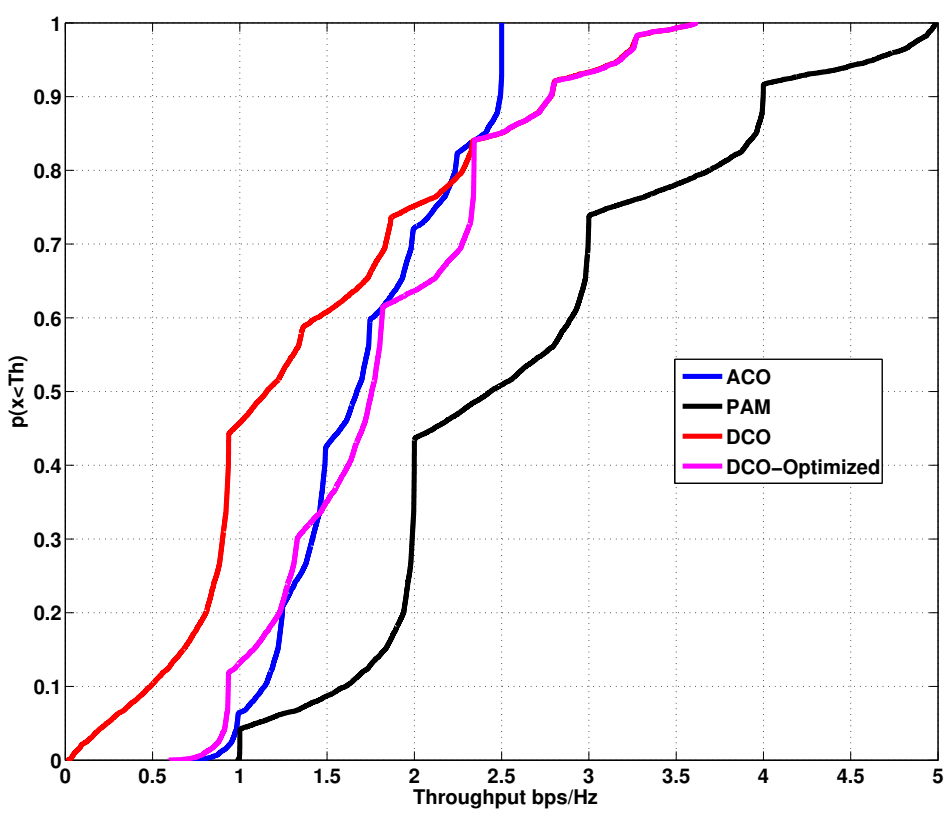

Figure 3.10: One beam CDF plot Vs throughput

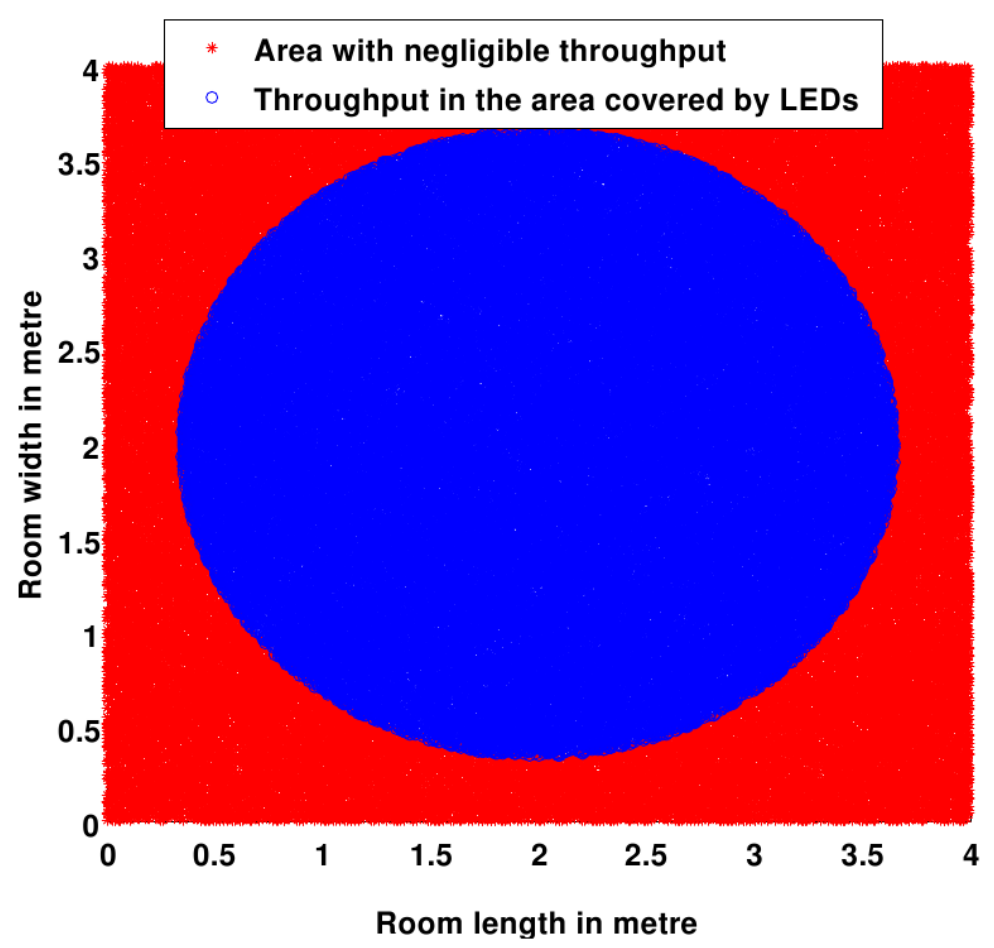

Figure 3.11: One beam throughput coverage area 


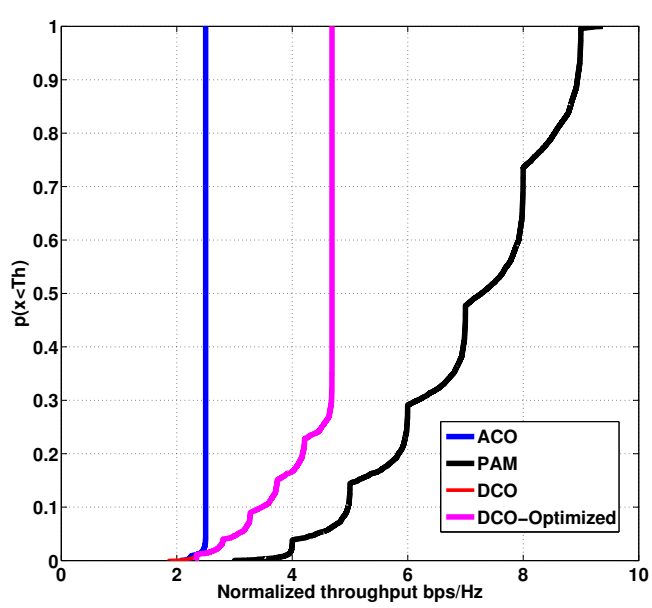

(a)

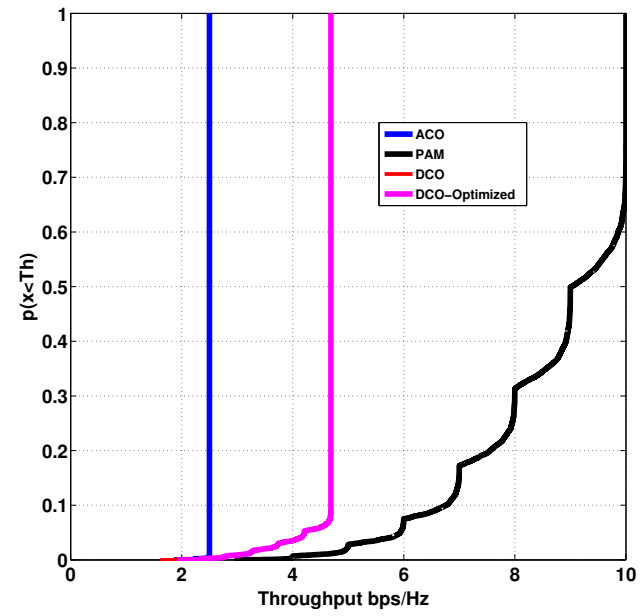

(b)

Figure 3.12: 4 cells CDF plot Vs throughput (a) one detector receiver (b) Four detectors receiver

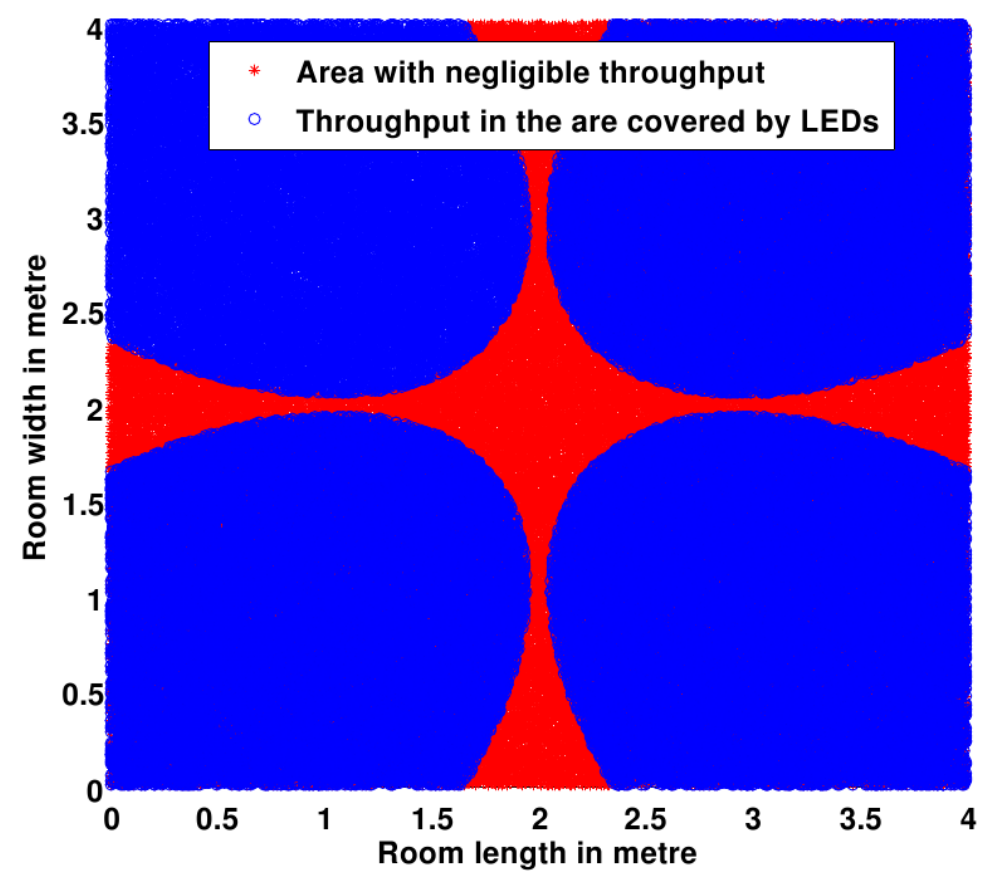

Figure 3.13: Four beam throughput coverage area

Figure 3.12 (a) shows the results of the CDF comparison between ACO, DCO, DCO-Optimized and PAM. Results for this scenario indicate that PAM provides a good performance compared to other schemes, also it can be seen that at higher modulation levels (from 64-QAM above) 
the DCO-13 dB and DCO optimized yield the same results. It also shows an improvement in throughput average when compared to one beam light. Looking at Figure 3.10 it can be seen that, the average throughput for ACO, DCO and PAM are $1.1 \mathrm{bps}, 1.57 \mathrm{bps}$ and $2.5 \mathrm{bps}$ respectively while for 4 cell systems Figure 3.12 (a) the average throughput results are 2.5 bps, 4.7 bps and 7 bps respectively. Figure 3.12 (b) shows the results of increasing the number of detectors at the receiver. The aim is to increase the chance of signal detection when either of the detectors points away from any of the 4 LEDs. In this setup the receiver has four detectors inclined in an elevation angle of $45^{\circ}$ and separated from each other by $90^{\circ}$. The receiver was allowed to orient randomly over a range of azimuth angles of $90^{\circ}$ for each detector which is equivalent to $360^{\circ}$ for the whole receiver (Figure 3.14), this is to simulate the random position of the receiving device as the user can place it in a different orientations. When more detectors were added in the receiver the results show that the throughput increases from 7 bps Figure 3.12 (a) to 9 bps Figure 3.12 (b) for PAM but remains the same for DCO and ACO because the schemes have reached their maximum possible theoretical throughput results and hit saturation at lower CDF value. However it is shown that in DCO the percentage of users that achieve maximum throughput has increased from $70 \%$ (Figure 3.12 (a)) to 90\% (Figure 3.12 (b)). Figure 3.13 depicts the four light beams coverage; here the coverage of each inclined LED and the overall coverage is shown. It shows that, distributing power to 4 LEDs and inclines them at a $45^{\circ}$ elevation angle causes beams of light to concentrate in a specific area hence reduces interference in the multiuser scenario, and also increases room coverage.

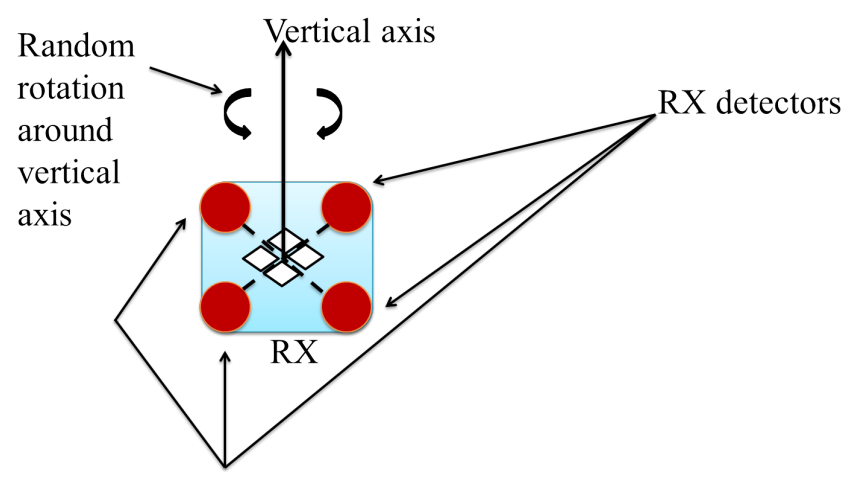

Figure 3.14: Four detectors receiver with random orientation 


\subsubsection{Multiuser multibeam simulation}

The setup in this simulation is similar to scenario in Figure 3.7 (b) with multiple users. The simulation considered the throughput calculations for separate cells and summed them to compute the capacity of the system, which is compared with a single user system. The aim is to distribute transmitter power, increase coverage and reduce interference. It this case it is assumed only one detector receiver. Figure 3.15 shows the results of simulating the ACO- multiuser scenario where the LEDs were set as in Figure 3.7 (b) and all receivers with one detector were pointing upward perpendicular to $\mathrm{x}, \mathrm{y}$ plane. Throughput calculations are performed based on Time Division Multiple Access (TDMA), i.e. using (3.31). In this scenario the impact of sharing four cells amongst multiple users is shown, when one by one setup was used (Figure 3.7 (a)), the average throughput was $1.71 \mathrm{bps}$, but the throughput increased to $2.5 \mathrm{bps}$ (saturated) after the power was distributed to four transmitting LEDs. For DCO, the throughput for one light beam was 1.75 bps but when scenario in Figure 3.7 (b) was considered i.e. the distribution of power to 4 cells the average throughput increased up to 4.7 bps as shown in Figure 3.16. When the cells are shared by number of users it is again shown that the system channel capacity increases with number of users to four times the single user capacity for both schemes. This is the maximum single user capacity multiplied by the number of cells in the system.

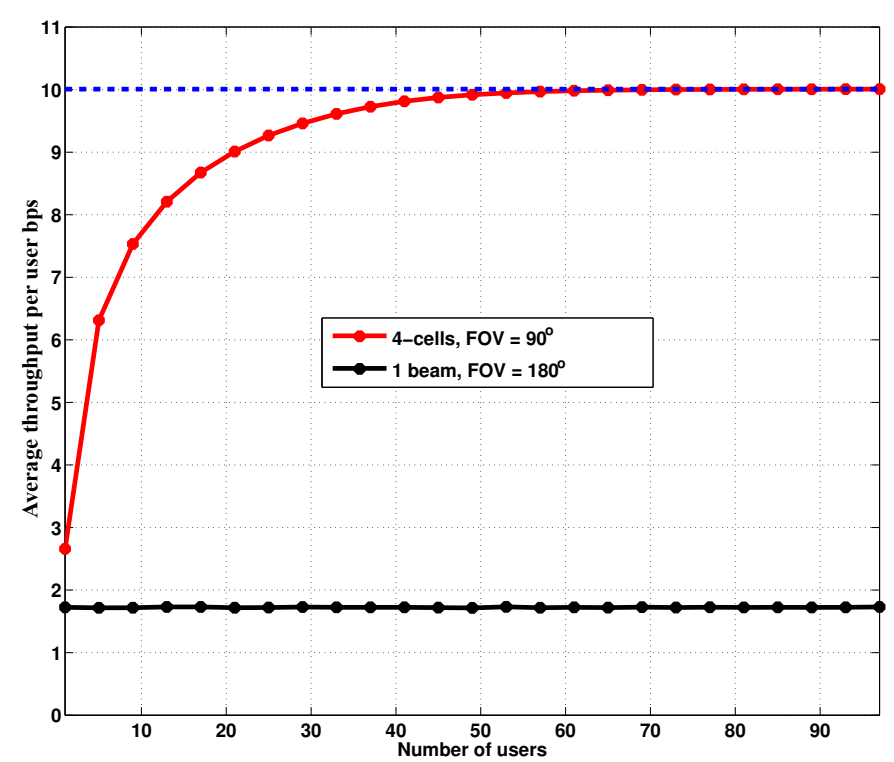

Figure 3.15: ACO average throughput Vs number of users 


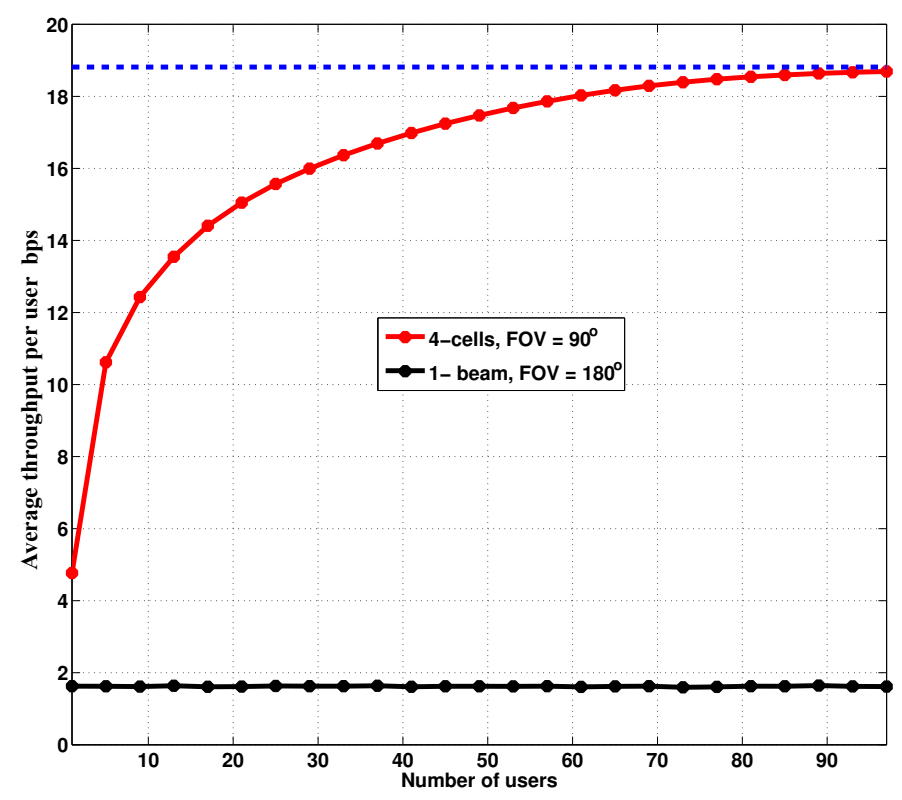

Figure 3.16: DCO average throughput Vs number of users

Figure 3.17 shows the results of simulating a PAM modulation-multiuser scenario with all receivers with one detector pointing upwards perpendicular to the $\mathrm{x}$, $\mathrm{y}$ plane. Throughput calculations was performed using equations (3.27) and (3.31). In this scenario the impact of sharing four cells amongst multiple users is shown, when one by one setup was used (Figure 3.7 (a)), the average throughput was 2.67 bps. In scenario two (Figure 3.7 (b)) the power is distributed by using four cells and the average throughput increased up to $7 \mathrm{bps}$. When the cells were shared by the number of users it is shown that the system channel capacity increases with number of users to nearly four times the single user capacity and it can be seen that for PAM more than 100 users must be accommodated for the system to reach throughput saturation. 


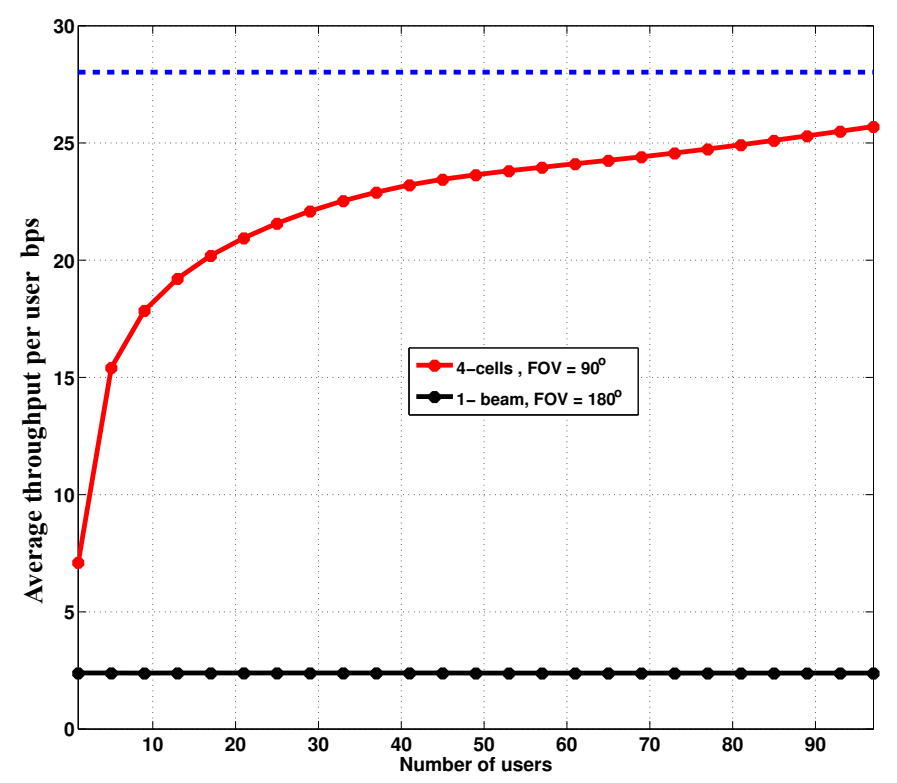

Figure 3.17: PAM average throughput Vs number of users

\subsection{Conclusion}

Diferent models are presented and different channel scenarios are simulated in indoor visible light communication. The performance of optical OFDM and single carrier in both single user and multiuser scenarios are compared. Our preliminary findings show that the four narrow cell beams can outperform one wide beam LED in terms of coverage area and hence average throughput. Looking at Figures 3.15, 3.16 and 3.17 It is shown that the capacity achieved by $180^{\circ}$ FOV single beam is $1.71 \mathrm{bps}, 1.75 \mathrm{bps}$, and $2.5 \mathrm{bps}$ while using $90^{\circ} \mathrm{FOV}$ four beams the system capacity increased to $2.5 \mathrm{bps}, 4.5 \mathrm{bps}$ and $7 \mathrm{bps}$ for ACO, DCO and PAM respectively. Also it is shown that sharing cells by number of users result in system channel capacity increase with number of usercoverage2s to four times the single user capacity (Figures 3.15, 3.16 and 3.17). This is the maximum single user capacity multiplied by the number of cells in the system. It was also noted that DCO optimized outperform DCO-13dB at the modulation levels lower than 64 QAM. Figure 3.10 shows that DCO-optimized gives the average throughput of 1.75 bps while DCO-13dB gives 1.1 bps. It was also seen that the optical receiver orientation has a big impact on the system throughput. The throughput improvement achieved after receiver orientation was about $20 \%$ over the fixed receiver (Figure 3.12). For the four cell case, ACO saturates in capacity at lower throughput values compared to DCO and PAM. 


\section{Chapter 4}

\section{Improved indoor VLC MIMO channel capacity using receiver with angular diversity detectors}

This chapter presents a receiver with angular diversity detector (A. detectors) using different MIMO modulation techniques. When evaluating the indoor OWC MIMO performance in the situation where the receiver is not stationary, the LOS between LEDs and PDs becomes an issue, especially in locations away from the vicinity of the LEDs. In such situations the reception and system throughput becomes poor due to the reduction of the channel matrix rank. This chapter studies the use of angular diversity detectors to overcome the problem and improve the system capacity. The chapter also proposes a MIMO transmission technique called adaptive spatial modulation (ASM) which incorporates adaptive modulation and coding (AMC). Per antenna rate control (PARC) techniques in SMP is also considered to ensure that the appropriate modulation is used according to the channel conditions. The chapter then provides a throughput evaluation at many points within the room and compares the performance of different techniques and scenarios as well.

\subsection{Introduction}

The parallel usage of multiple transmitters and receivers e.g. multiple input multiple output (MIMO) can enhance the overall system performance and hence spectral efficiency and also reduce the bit error ratio (BER) of a communication system [8, 16,94]. Also MIMO systems can realize high speed transmission without increasing transmit power and or bandwidth. MIMO techniques have been applied to free space optical communications and data transmission to a limited extent. High data rate MIMO optical wireless communications using white LEDs were proposed in [8], where a fixed receiver with an imaging lens was used. It was found that the imaging lens and detector array size are physically large for some congurations and may not be practical for some applications. Mesleh et al. proposed indoor MIMO optical wireless 
communications using spatial modulation (SM) and concluded that reducing both the distance between the transmitter (TX) and the receiver (RX) and transmitter emerging angle (angle between the TX axis and the straight line to the receiver) results in lower correlation [16]. Optical MIMO for short range free space data transmission was proposed in [94] and it was shown that MIMO has high potential to improve spectral efficiency. An indoor optical wireless collaborative cellular system was proposed in [17]. In the paper, base stations and portable devices were designed, presented and analysed. Base stations (BS) located on a number of rings were proposed to cover a wide area, however the MIMO techniques did not perform properly at all receiver positions due to symmetry problem. In addition to that, the analysis of the throughput of the system was considered as future work. The use of angular diversity for the receiver was studied in $[18,95]$ for single input multiple output (SIMO) setup. In these papers multiple photodiodes (PDs) were used and a proper signal combining scheme were used to optimize the system performance. The results revealed that the angular diversity receiver outperforms the single-PD receiver with regard to signal to interference and noise ratio (SINR).

In [6] the performance comparison of MIMO techniques for optical wireless communications in the indoor environment were proposed and the results show that SMP improves the spectrum efficiency where there is low channel correlation effects. It was also shown that SM is competitive at low spectral efficiency while SMP performs better in high spectral efficiency as SM needs large signal constellation size to provide the same efficiency. Also SM is more robust to channel correlation. Repetition Coding (RC) was found to be insensitive to different transmitter-receiver alignments, but it needs a large signal constellation size to provide high data rates. In [96] two decision techniques that interchange between MIMO and diversity schemes for improving shadowing and alignment problems was proposed. The results suggested that shadowing and alignment problems commonly encountered in visible light communication (VLC) systems can be readily solved using these methods.

Saha et al. proposed a receiver diversity method in [97] in order to mitigate performance variation, the method aimed at improving VLC receiver performance. The results demonstrated performance improvement in terms of received power and bit error rate. Biagi et al. exploited OWC MIMO techniques jointly with pulse position modulation (PPM) to perform space block coding and achieved data rates of the order of hundreds of $\mathrm{Mb} / \mathrm{s}$ (i.e., around $600 \mathrm{Mb} / \mathrm{s}$ ) in indoor VLC systems. In [98] the experimental demonstration of an indoor visible light nonimaging MIMO system with an aggregate error free bit rate of $50 \mathrm{Mb} / \mathrm{s}$ over a distance of 
$2 \mathrm{~m}$ was reported. The system used four independent white LED transmitters, each transmitting 12.5 Mb/s of data in the ON-OFF keying non-return-to-zero format, with four independent nonimaging optical receivers. The experiments have shown that each pair of Tx and Rx in the MIMO system outperforms SISO. In [99], a novel OWC imaging diversity scheme in a MIMOVLC system was analysed. According to the simulation results it was advised to use a lens with FOV not more than $60^{\circ}$ at the receiver for better separation between detectors.

In [100] Tran et al. evaluated RC and SMP applied to VLC in indoor scenarios with non-direct line of sight (LOS) characteristics. The optimal values of transmitter separation for RC and SMP are $1.5 \mathrm{~m}$ and $3.5 \mathrm{~m}$, respectively. The results showed that RC outperforms SMP at low spectral efficiency. However, in high spectral efficiency RC does not perform well since it requires a very high constellation size, while SMP with its spatial multiplexing gain can offer a much better performance. An imaging receiver scheme for an indoor MIMO VLC, in which a fisheye lens with an ultrawide FOV is used for high quality imaging was proposed in [101], where high spatial diversity for decoding of the MIMO signals was provided. The simulation results indicated that low correlations of the channel matrix were achieved, so high spectral efficiency is realized with various receiver positions in indoor VLC setup.

In all these discussed papers, the movement of the receiver to different locations to see how it affects channel correlation and hence spectral efficiency was not covered. In this chapter, a mobile receiver with angular diversity detectors in VLC MIMO channels is considered. The objective is to improve the rank of the channel matrix and hence system throughput. RC, SMP and SM concepts are used to evaluate throughputs across multiple locations in a small room scenario. Since the receiver is mobile, the channel gains are weak in some locations of the room due to the lack of line of sight (LOS) paths between transmitters and receivers. Therefore AMC and PARC are used to improve spectral efficiency. In PARC, see [102], the choice of modulation is optimized separately for each transmitting antenna (LED). The throughputs for vertically oriented detectors (V. detectors) are compared with the oriented/inclined detectors for different cases. The results show that for mobile receivers, oriented/inclined detectors with AMC and PARC provide capacity improvement when compared with the vertically oriented receivers. 
This chapter gives the following novel contributions:

- Develop angular diversity optical detectors in a MIMO configuration to provide LOS channel conditions in more locations of the room and compare their performance with that of vertical detectors.

- Devise a novel adaptive form of SM called Adaptive Spatial Modulation (ASM) which is robust to rank deficient channels in different locations of the room

- Provide performance statistics for MIMO methods operating over many room locations, using AMC and PARC concepts at the transmitter.

\subsection{System model}

An indoor $4 \times 4$ MIMO scenario $\left(N_{r}=4\right.$ and $\left.N_{t}=4\right)$ as in [47] is considered. The system is located within a room of size $4 \times 4 \times 3 \mathrm{~m}$ and it is assumed the transmitters are placed at a height of $2.50 \mathrm{~m}$ and oriented downwards perpendicular to the floor of the room. The receiver is allowed to move randomly at a height of $0.75 \mathrm{~m}$ (human waist or table height) and its detectors oriented in variable elevation angles as in Figure 4.1 (c). The inclined detector's orientation is meant to increase the FOV between transmitters and receivers and hence increase system spectral efficiency.

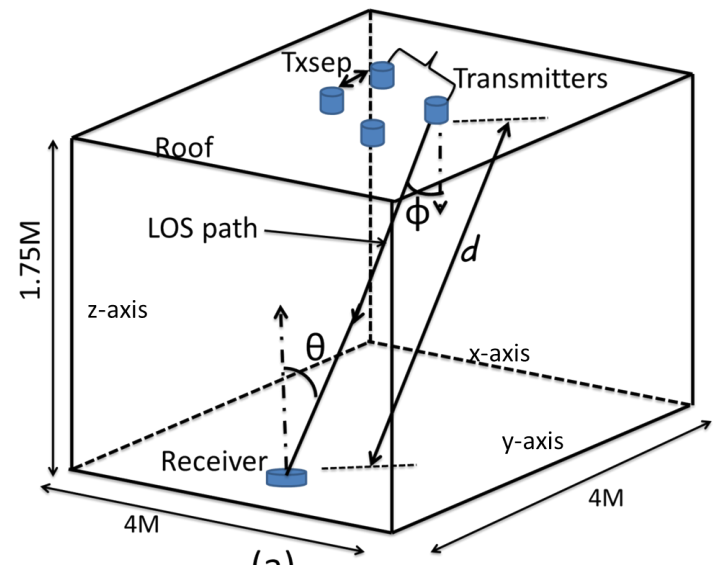

(a)

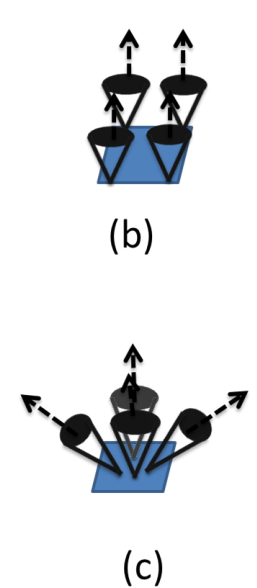

(c)

Figure 4.1: Transmitter and receiver geometry used for channel gains calculations (a) General setup (b) Receiver with vertical axis detectors (c) Receiver with inclined axis detectors 
VLC MIMO transmission is considered where Intensity Modulation (IM) and Direct Direction (DD) of the optical carrier using incoherent light source are employed. The system consists of $N_{t}$ transmitters and $N_{r}$ photo-detectors at the receiver side. The received signal vector is:

$$
\mathbf{y}=\mathbf{H s}+\mathbf{n}
$$

where $\mathbf{s}$ is the transmitted signal vector which is defined as follows: $\mathbf{s}=\left[s_{1} \ldots \ldots . s_{N t}\right]^{T}$ with $[.]^{T}$ being the transpose operator and $s_{n}$ denotes the signal transmitted by $n^{\text {th }}$ LED. The sum of ambient shot light noise and thermal noise is denoted by an $N_{r} \times 1$ vector $\mathbf{n}$ which is assumed to be real valued additive white Gaussian noise (AWGN) with zero mean and variance:

$$
\delta^{2}=\delta_{\mathrm{ss}}^{2}+\delta_{\mathrm{js}}^{2}
$$

where $\delta^{2}$ ss is the shot noise variance (3.7) and $\delta^{2}{ }_{\mathrm{js}}$ is the thermal noise variance (3.10). Thus the noise power is given by $\delta^{2}=N_{0} B$, where $N_{0}$ is the noise power spectral density and $B$ is bandwidth. The $N_{r} \times N_{t}$ channel matrix $\mathbf{H}$ for each room coordinate $(x, y, z)$ is given by:

$$
\mathbf{H}(x, y, z)_{L O S}=\left(\begin{array}{ccccc}
h_{11}(x, y, z) & h_{12}(x, y, z) & . & . & h_{1 N t}(x, y, z) \\
h_{21}(x, y, z) & h_{22}(x, y, z) & & \cdot \\
\cdot & & \cdot & \cdot \\
\cdot & & & \cdot & \cdot \\
h_{N r 1}(x, y, z) & . & \cdot & \cdot & h_{N r N t}(x, y, z)
\end{array}\right)
$$

where $h_{n_{r} n_{t}}$ represents the channel transfer function of the wireless link between transmitter $n_{t}$ and receiver $n_{r}$ at location $(x, y, z)$. The path difference between multiple transmitter-receiver links is, typically a few $\mathrm{cm}$ as shown in Figure 4.1. Therefore, the delay between the different links is negligible. Consequently we consider that the delay spread of the channel is much smaller than the symbol period. In this chapter we assume an optical wireless link with LOS characteristics (Figure 4.1). As illustrated $\phi$ is the angle of emergence with respect to the transmitter axis, $\theta$ is the angle of incidence with respect to the receiver/detector axis and $d$ is the distance between transmitter and receiver.In the figure, TxSep denotes the transmitters separation. Consider a LOS optical system propagation path in Figure 4.1, the channel gain from the transmitter to the receiver is given as: 


$$
h=\left\{\begin{array}{cc}
\frac{A(\kappa+1) \cos ^{\kappa}(\phi) \cos (\theta)}{2 \pi d^{2}}, & 0 \leq \theta \leq \varphi_{\frac{1}{2}} \\
0, & \theta>\varphi_{\frac{1}{2}}
\end{array}\right.
$$

where, $\kappa=\frac{-\ln (2)}{\ln \left(\cos \left(\Phi_{\frac{1}{2}}\right)\right)}$. The scalar $A$ is the collection area of the detector, $\Phi_{\frac{1}{2}}$ is the transmitter semi-angle (at half power) and the scalar $\varphi_{\frac{1}{2}}$ is the field of view (FOV) semi angle of the receiver.

Figure 4.1 (c) shows the receiver with inclined detectors. To achieve this setup the detector axis vector $\mathbf{z}$, which is inclined in an elevation angle $45^{\circ}$, is transformed around the $z$ axis (vertical axis) using the transformation matrix such that:

$$
\begin{aligned}
& \hat{\mathbf{z}}=\quad \mathbf{R}_{\mathbf{z}}(\vartheta+\omega) \quad \times \mathbf{z} \\
& \left(\begin{array}{l}
\hat{x} \\
\hat{y} \\
\hat{z}
\end{array}\right)=\left(\begin{array}{ccc}
\cos (\vartheta+\omega) & -\sin (\vartheta+\omega) & 0 \\
\sin (\vartheta+\omega) & \cos (\vartheta+\omega) & 0 \\
0 & 0 & 1
\end{array}\right)\left(\begin{array}{l}
x \\
y \\
z
\end{array}\right)
\end{aligned}
$$

where $\hat{\mathbf{z}}$ is the transformed vector, $\vartheta$ presents the transformation angle around the $\mathbf{z}$ axis for separation of $90^{\circ}$ to form detectors' axis vectors at azimuth angles $0^{\circ}, 90^{\circ} 180^{\circ}$ and $270^{\circ}$ with an addition random rotation angle $\omega$. The angle $\omega$ is uniformly distributed between $0^{\circ}$ to $360^{\circ}$ and $\mathbf{R}_{\mathbf{z}}$ is the transformation matrix with respect to $\mathbf{z}$ axis. Since any variation of detector's axis affects the angle of incidence $\theta$ as in equation (4.4), each detector elevation angle is varied from $45^{\circ}$ to $90\left(0^{\circ}\right.$ from vertical $)$ to find the elevation angle that will maximize the rank of channel matrix $\mathbf{H}$. Therefore the selected angle is used for system performance evaluation at each location.

\subsection{MIMO techniques}

Three different MIMO techniques are used; RC, SM and SMP as in [47]. In contrast to [8], [16] and [94] where fixed receivers are used, in this chapter it is considered that the mobile receiver is able to move freely around the room. To overcome the resulting SNR variations AMC with PARC in SMP is employed and AMC in SM and RC. In these techniques the modulation $M$ level is chosen and updated in each transmit time depending on the current channel conditions 
i.e the computed SNR at the receiver is fed back periodically to the transmitter and the transmitter chooses the appropriate modulation level (Figure 4.2). This feedback can be achieved by employing infrared LEDs for uplink transmission. It is assumed that all considered MIMO

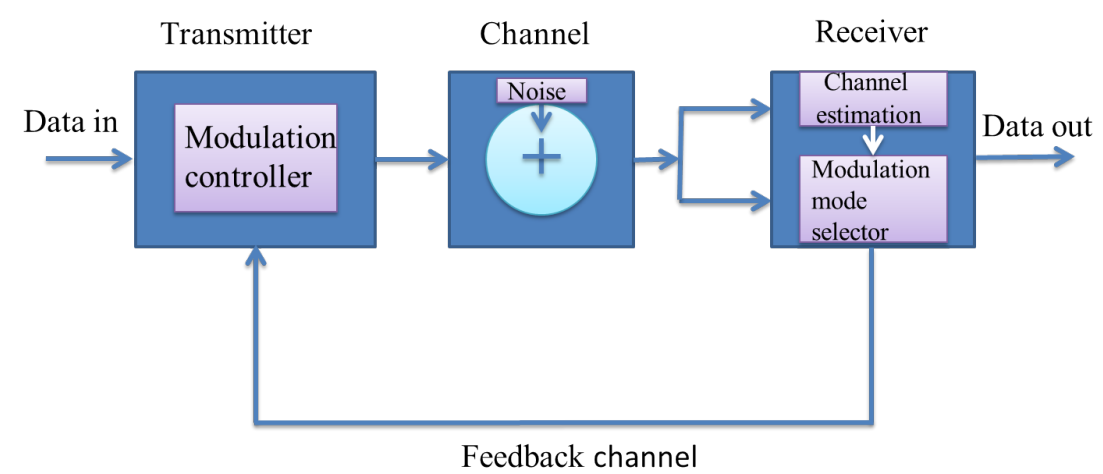

Figure 4.2: Block diagram illustrating adaptive modulation

techniques use Maximum Likelihood (ML) [47] detection at the receiver with perfect knowledge of the channel and ideal time synchronisation, except for SMP where the zero forcing (ZF) decoding technique was used to avoid excessive complexity and computation time when using the PARC method. Therefore the decoder selects a constellation vector $\widetilde{\mathbf{s}}$, which minimizes the Euclidean distance between the actual received signal $\mathbf{y}$ and all the possible signal vectors leading to:

$$
\widetilde{\mathbf{s}}=\underset{\mathbf{s}}{\arg \max } p_{\mathbf{y}}(\mathbf{y} \mid \mathbf{s}, \mathbf{H})=\underset{\mathbf{s}}{\arg \min }\|\mathbf{y}-\mathbf{H} \mathbf{s}\|_{F}^{2}
$$

where $p_{\mathbf{y}}$ is the probability density function of $\mathbf{y}$ conditioned in $\mathbf{s}$ and $\mathbf{H}$. $\|\cdot\|_{F}$ denotes the Frobenius norm.

\subsubsection{Repetition Coding (RC)}

The first technique used in this chapter is RC which simultaneously emits the same signal from all transmitters (Figure 4.3). Therefore the condition $s_{1}=s_{2}=\ldots . . s_{N t}$ holds [47]. In $\mathrm{RC}$ the intensity coming from the several transmitter constructively adds up at the receiver side. In this chapter unipolar $M$-level Pulse Amplitude Modulation ( $M$-PAM) is considered together with RC, where $M$ denotes the signal constellation size. Therefore $M$-PAM achieves a spectral efficiency of $\log _{2}(M) \mathrm{bit} / \mathrm{s} / \mathrm{Hz}$. PAM is more bandwidth efficient compared to other pulse modulation techniques like Pulse-width Modulation (PWM), On-off Keying (OOK) and 


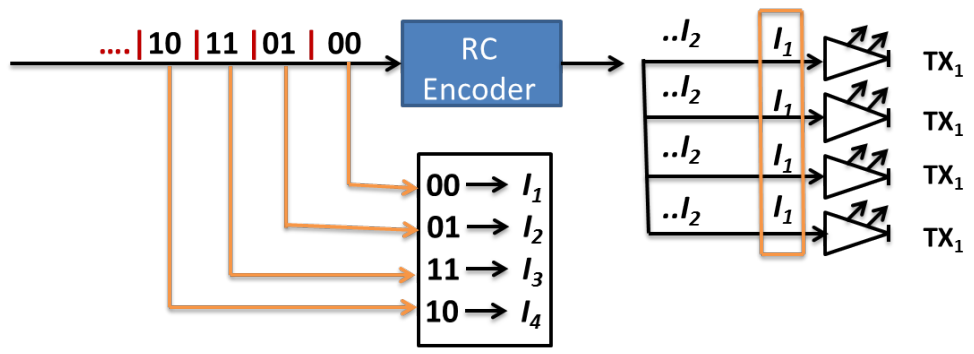

Figure 4.3: Illustration of $R C$ operation with $N_{t}=4$ and $M=4$. Simultaneously emits the same signal from all transmitters

Pulse-Position Modulation (PPM). [47]. Rectangular pulse shapes with $M$-PAM is employed. The intensity level emitted by the Light Emitting Diode (LED) is given by:

$$
I_{m}^{\mathrm{PAM}}=\frac{2 I}{M-1} m, \text { for } m=0,1, \ldots \ldots . .(M-1)
$$

where $I$ is the mean optical power emitted. BER for unipolar $M$-PAM can be expressed by

$$
\mathrm{BER}_{\mathrm{PAM}} \geq \frac{2(M-1)}{M \log _{2}(M)} Q\left(\frac{1}{M-1} \sqrt{\frac{E_{R X}}{N_{0}}}\right)
$$

where:

$$
Q(a)=\frac{1}{\sqrt{2 \pi}} \int_{a}^{+\infty} \exp \left(\frac{-t^{2}}{2}\right) d t
$$

is the $Q$ function [47] and $E_{R X}$ is the received electrical energy. The BER of $M$-PAM given in (4.8) can be generalized for the $N_{r} \times N_{t}$ scenario. The BER of RC is given in equation (7) of [47] as

$$
\mathrm{BER}_{\mathrm{RC}} \geq \frac{2(M-1)}{M \log _{2}(M)} Q\left(\frac{1}{M-1} \sqrt{\frac{E_{s}}{N_{0} N_{t}^{2}} \sum_{n_{r}=1}^{N_{r}}\left(\sum_{n_{t}=1}^{N_{t}} h_{n_{r} n_{t}}\right)^{2}}\right)
$$

Where, $E_{s}=(\varrho I)^{2} T_{s}$ denotes the mean emitted electrical energy of the intensity modulated optical signals. $\varrho$ represents the optical to electrical conversion coefficient. It is assumed that, $\varrho=1 \mathrm{~A} / \mathrm{W}$. The scalar $T_{s}$ denotes symbol duration in seconds and $\mathrm{A}$ is the unit current in amperes. 


\subsubsection{Spatial Modulation (SM)}

The second used technique is SM, which combines MIMO and digital modulation proposed in [103] and further investigated in [104-106]. In SM the conventional constellation diagram is extended to an additional dimension namely, the spatial dimension. Specifically, the LED index is used to transmit bits. Each transmitting LED is assigned a unique binary sequence (the spatial symbol) (Figure 4.4). A transmitter is only activated when the random spatial symbol to be transmitted matches the pre-located spatial symbol. Thus only one transmitter is activated

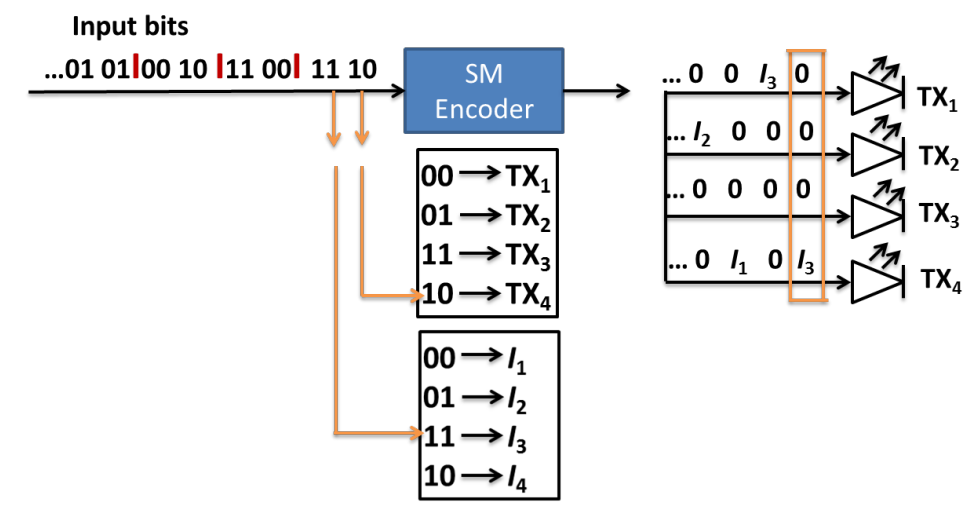

Figure 4.4: Illustration of SM operation with $N_{t}=4$ and $M=4$. The first two bits in the block of four bits determine the PAM symbol and second two bits determine the active LED [47]

for any PAM symbol transmission. Therefore only one element of the signal vector $s$ to be transmitted is non-zero. SM provides an enhanced spectral efficiency of $\log _{2}\left(N_{t}\right)+\log _{2}(M)$ $\mathrm{bit} / \mathrm{s} / \mathrm{Hz}$. Signals with intensity $I_{m}=0$ can not be used for the signal modulation of SM because in this case no transmitter would be active and the spatial information information would be lost [47]. Therefore, the intensities of common PAM given in (4.7) has to be modified to suit SM, leading to:

$$
I_{m}^{\mathrm{SM}}=\frac{2 I}{M+1} m, \text { for } m=1, \ldots \ldots . .(M)
$$


BER expression for SM is given in equation (13) of [47] as:

$$
\begin{array}{r}
\mathrm{BER}_{\mathrm{SM}} \leq \frac{1}{M N_{t} \log _{2}\left(M N_{t}\right)} \sum_{m^{(1)}=1}^{M} \sum_{n_{t}^{(1)}=1}^{N_{t}} \sum_{m^{(2)}=1}^{M} \sum_{n_{t}^{(2)}=1}^{N_{t}} \mathrm{~d}_{\mathrm{H}}\left(b_{m^{(1)} n_{t}^{(1)}}, b_{m^{(2)} n_{t}^{(2)}}\right) \\
Q\left(\sqrt{\frac{E_{s}}{4 N_{0}} \sum_{n_{r}=1}^{N_{r}}\left|I_{m^{(2)}}^{\mathrm{SM}} h_{n_{r} n_{t}^{(2)}}-I_{m^{(1)}}^{\mathrm{SM}} h_{n_{r} n_{t}^{(1)}}\right|^{2}}\right)
\end{array}
$$

Where, $\mathrm{d}_{\mathrm{H}}\left(b_{m^{(1)}}, b_{m^{(2)}}\right)$ denotes the Hamming distance of two bit assignments $b_{m^{(1)}}$ and $b_{m^{(2)}}$ of the signal vectors $\mathbf{s}_{m^{(1)}}$ at the transmitter and signal $\mathbf{s}_{m^{(2)}}$ at the receiver.

\subsubsection{Adaptive Spatial Modulation (ASM)}

The third technique is ASM which is a modified form of spatial modulation (SM). ASM is proposed in this chapter to cope with rank deficient channels due to the fact that, when the receiver moves around the room the channel matrix $\mathbf{H}$ is not full rank (4) in many locations [107]. If the channel matrix rank is reduced, the receiver can not easily distinguish all the transmitting LEDs. By using antenna selection techniques, the ASM receiver checks the rank of the channel matrix and decides which TX/RX setup to be used.

The authors in [108-110] proposed some different techniques for antenna selection. This chapter will discus the methods proposed in [110] which is relevant to the technique proposed in this chapter. In [110] they use two methods which reduce the order of complexity (number of times the optimization metric is evaluated). The first method was a Euclidean distance optimized antenna selection (EDAS) which chooses the specific antenna set that maximizes the minimum Euclidean distance among all the possible transmit vectors. The second one was capacity optimized antenna selection (COAS) which uses the bounded system capacity to chooses the set of antennas corresponding to the largest channel norms out of number of transmitting antennas. In this chapter a similar method to COAS is used but with the difference that the system capacity is not used as the metric as it is not applicable to positive real channels that arise in OWC. Instead the channel matrix rank is used here to decide the number of antennas to be active, thereafter the largest channel norms will be used to decide the set of antennas to be used. The following three steps are involved. 
- If $\operatorname{rank}(\mathbf{H})=4$, four transmitter Adaptive Modulation SM is used for transmission and throughput calculations.

- If $\operatorname{rank}(\mathbf{H})=2$ or 3, 2TX Adaptive Modulation SM is employed. To identify which two transmitters are to be activated the $\mathbf{H}$ matrix is checked using the norm based method (4.14). Assume $\mathbf{q}_{\mathbf{n}_{\mathbf{t}}}$ is the column of $\mathbf{H}$ as follows:

$$
\mathbf{q}_{\mathbf{n}_{\mathbf{t}}}=\left(\begin{array}{c}
h_{1 n_{t}} \\
\cdot \\
\cdot \\
\cdot \\
h_{N r n_{t}}
\end{array}\right), n_{t}=1, \ldots \ldots, N_{t}
$$

Then two highest norm of sum of each $\mathbf{q}_{\mathbf{n}_{\mathbf{t}}}$ values are selected such that the selected two norms will present the column of transmitters $T X_{1}$ and $T X_{2}$ :

$$
\operatorname{Norm}_{n_{t}}=\sum_{n_{r}=1}^{N_{r}}\left|h_{n_{r} n_{t}}\right|, n_{t}=1 \ldots \ldots N_{t}
$$

then the norms are sorted in decreasing order so that the first two largest norms identify transmitters to be selected.

- If $\operatorname{rank}(\mathbf{H})=1$, w RC with Adaptive Modulation is used.

If $\Omega$ denotes the number of selected antennas, the BER of ASM is approximated using the joint BER evaluation method. This uses both the SM and the RC BERs as used in [47], which are jointly evaluated as follows : 


$$
\mathrm{BER}_{\mathrm{ASM}} \leq\left\{\begin{array}{cl}
\frac{1}{M \Omega \log _{2}(M \Omega)} \sum_{m^{(1)}=1}^{M} \sum_{n_{t}^{(1)}=1}^{\Omega} \sum_{m^{(2)}=1}^{M} \sum_{n_{t}^{(2)}=1}^{\Omega} \mathrm{d}_{\mathrm{H}}\left(b_{m^{(1)} n_{t}^{(1)}}, b_{m^{(2)} n_{t}^{(2)}}\right) . & \\
Q\left(\left.\sqrt{\frac{\varrho^{2} T_{s}}{4 N_{0}} \sum_{n_{r}=1}^{N_{r}} \mid I_{m^{(2)}}^{\mathrm{SM}} h_{n_{r} n_{t}^{\prime}}^{(2)}-I_{m^{(1)}}^{\mathrm{SM}} h_{n_{r} n_{t}^{\prime}}^{\prime(1)}}\right|^{2}\right), & 2 \leq \Omega \leq 4 \\
\frac{2(M-1)}{M \log _{2}(M)} Q\left(\frac{1}{M-1} \sqrt{\frac{E_{s}}{N_{0} N_{t}^{2}} \sum_{n_{r}=1}^{N_{r}}\left(\sum_{n_{t}=1}^{N_{t}} h_{n_{r} n_{t}}\right)^{2}}\right), & \Omega=1
\end{array}\right.
$$

In this equation $h_{n_{r} n_{t}}^{\prime}$ denotes the channel gains of the selected antennas.

\subsubsection{Spatial Multiplexing (SMP)}

The final MIMO technique is SMP. In SMP independent data streams are simultaneously transmitted from all the transmitters (Figure 4.5). Since ZF is used to estimate the transmitted symbol in SMP, the following equation is used to obtain the estimate value of $\mathbf{s}$ :

Consider equation (4.1),

$$
\widetilde{\mathbf{s}}=\mathbf{W} \cdot \mathbf{y}
$$

where, $\mathbf{W}$ denotes the Pseudo-inverse of channel matrix $\mathbf{H}$ which is given by:

$$
\mathbf{W}=\left(\mathbf{H}^{T} \mathbf{H}\right)^{-1} \mathbf{H}^{T}
$$

SMP provides a maximum spectral efficiency of $N_{t} \log _{2}(M) \mathrm{bit} / \mathrm{s} / \mathrm{Hz}$.

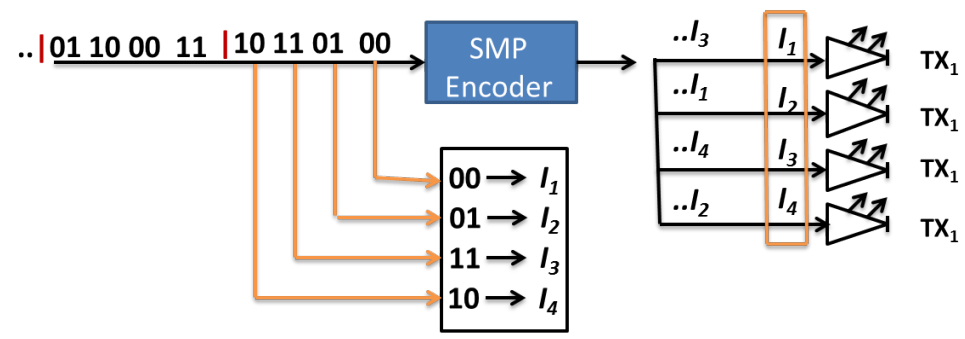

Figure 4.5: Illustration of SMP operation with $N_{t}=4$ and $M=4$. Independent data streams are simultaneously transmitted from all the transmitters

As for RC, PAM is used with SMP and equally distributes the optical power across all emitters to ensure that both schemes use the same transmit power. The BER for SMP is given as: 


$$
\mathrm{BER}_{\mathrm{SMP}} \leq \frac{1}{N_{t}} \sum_{i=1}^{N_{t}} \frac{2\left(M_{i}-1\right)}{M_{i} \log _{2}\left(M_{i}\right)} Q\left(\frac{1}{M_{i}-1} \sqrt{\frac{E_{s}}{N_{0} N_{t}^{2}\left\|\mathbf{W}_{i}\right\|^{2}}}\right)
$$

where, $\mathbf{W}_{i}$ denotes $i^{\text {th }}$ row of pseudo-inverse of channel matrix $\mathbf{H}, M_{i}$ is the $i^{\text {th }}$ selected modulation level for transmitter LED $i$.

\subsection{System throughput evaluation}

For the RC techniques the BER Expression described in equation (4.10) is used and the throughput calculation method given by [18] is used:

$$
T_{h(\mathrm{SISO})}=R(1-\gamma) \mathrm{bps} / \mathrm{Hz}
$$

Where $R$ is the maximum rate of the scheme and $\gamma$ is the packet error probability which is given by [18]:

$$
\gamma=1-(1-B E R)^{N_{b}}
$$

Where, $N_{b}$ is the number of bits in one packet. Equation (4.19) is computed for all modulation sizes $M$ which yield a BER less than $10^{-2}$ (see Figure 3 of [19]). Then, the highest throughput determines the modulation scheme that is selected. A BER greater values than $10^{-2}$ is ignored since will not give a substantial throughput gain as shown in Figure 3. of [19].

For SM, the BER calculations is carried out using equations (4.12) and the technique for selecting the best BER is the same as in RC. The verall throughput is calculated using the following equation:

$$
T_{h(\mathrm{MIMO})}(x, y, z)=T h_{\mathrm{BER}_{M i}}
$$

For ASM, after the process explained in subsection (c) of (III) has been performed, the BER calculations is carried out based on equation (4.15), and also choose the highest modulation level which gives the highest throughput such that the BER is less than $10^{-2}$. The overall 
throughput is calculated using the following equation:

$$
T_{h(\mathrm{MIMO})}(x, y, z)=T h_{\mathrm{BER}_{N_{T M}}}
$$

Where, $N_{T}$ denotes number of selected transmitters (LEDs) in a given channel rank condition. For SMP the similar approach as in RC is used except that, in this case adaptive modulation with PARC [102] is used to optimize the choice of modulation separately for each transmitter. For each possible set of modulation schemes, the BER expression as in equation (4.18) is computed and choose the highest modulation level which gives the highest throughput such that the BER is less than $10^{-2}$ :

$$
T_{h(\mathrm{MIMO})}(x, y, z)=\sum_{i=1}^{N_{T}} T h_{\mathrm{BER}_{\operatorname{TX}_{i M i}}}
$$

Where $T h_{\mathrm{BER}_{\mathrm{TX}_{i}{ }_{i} i}}$ denotes throughput for an individual LED with an appropriate modulation while $N_{T}$ presents the number of transmitting LEDs.

\subsection{Simulation parameters and results}

The simulation parameters are given in Table 4.1. The room size shows the dimension of the room where the simulation is assumed.

\begin{tabular}{|c|c|}
\hline \multicolumn{1}{|c|}{ Parameters } & Values \\
\hline Room size $(W \times L \times H)$ & $4 \mathrm{~m} \times 4 \mathrm{~m} \times 3 \mathrm{~m}$ \\
\hline TX/RX setup & $4 / 4$ \\
\hline TX separations & $0.2 \mathrm{~m}, 0.4 \mathrm{~m}, 0.6 \mathrm{~m}$ \\
\hline RX separations (V. detectors) & $0.1 \mathrm{~m}$ \\
\hline TX/RX FOV & $45^{\circ}, 45^{\circ}$ \\
\hline RX elevation angle (A. Diversity) & $45^{\circ}$ to $90^{\circ}$ towards vertical \\
\hline RX azimuth angle separation (A. Diversity) & $90^{\circ}$ \\
\hline Photodiode area $(A)$ & $1 \mathrm{~cm}{ }^{2}$ \\
\hline MIMO transmission techniques & RC, SMP, SM \\
\hline Modulation schemes & 2 PAM-1024PAM \\
\hline
\end{tabular}

Table 4.1: Parameters used for simulation 
TX/RX setup presents the number of transmitters and receivers used in MIMO configuration. TX separations shows how the four transmitting LEDs are separated from each other. RX separation shows how the receiver detectors are separated (this applies for vertically oriented receiver). TX/RX FOV are the Field of View angles of transmitter and receiver respectively. $\mathrm{RX}$ detectors elevation angle is the angle between the detector's axis vector and vertical axis. For vertically oriented detectors the angle of elevation is zero. RX detectors azimuth angle separations shows how four detectors are separated in azimuth around $360^{\circ}$ (for A. Detectors). As for the vertically oriented receiver (V. Detectors) the angle is always zero from the vertical.

\subsubsection{Vertical detectors setup}

The setup developed in [47] was repeated for all proposed MIMO transmission techniques and different TX separations $(0.2 \mathrm{~m}, 0.4 \mathrm{~m}, 0.6 \mathrm{~m})$. RX detectors' separation remains $0.1 \mathrm{~m}$ all of the time with a vertically oriented RX as in Figure 4.2 (b), assuming installation of the detector array in typical laptops. The BER results given in Figure 4.6 match closely with Figure 3(a) of [47] and verify the validity of the simulator. The large SNR values in Figure 4.6 indicate the SNR compensation caused by $80 d B$ loss. This is because the optical channel gains have values interms of $10^{-} 6$ [47]. The mobile receiver model was then developed and the throughput for 1000 locations was evaluated. In this case adaptive modulation is used for all of the transmission techniques, i.e. the rate of transmission is updated periodically depending on channel condition. In addition to adaptive modulation, PARC in SMP is considered. The results of average throughputs for different transmission techniques and different TX separations are compared in Table 4.2 and CDF for both techniques are in Figure 4.10. Both Table data and CDF plots are explained in subsection 4.5.3. 


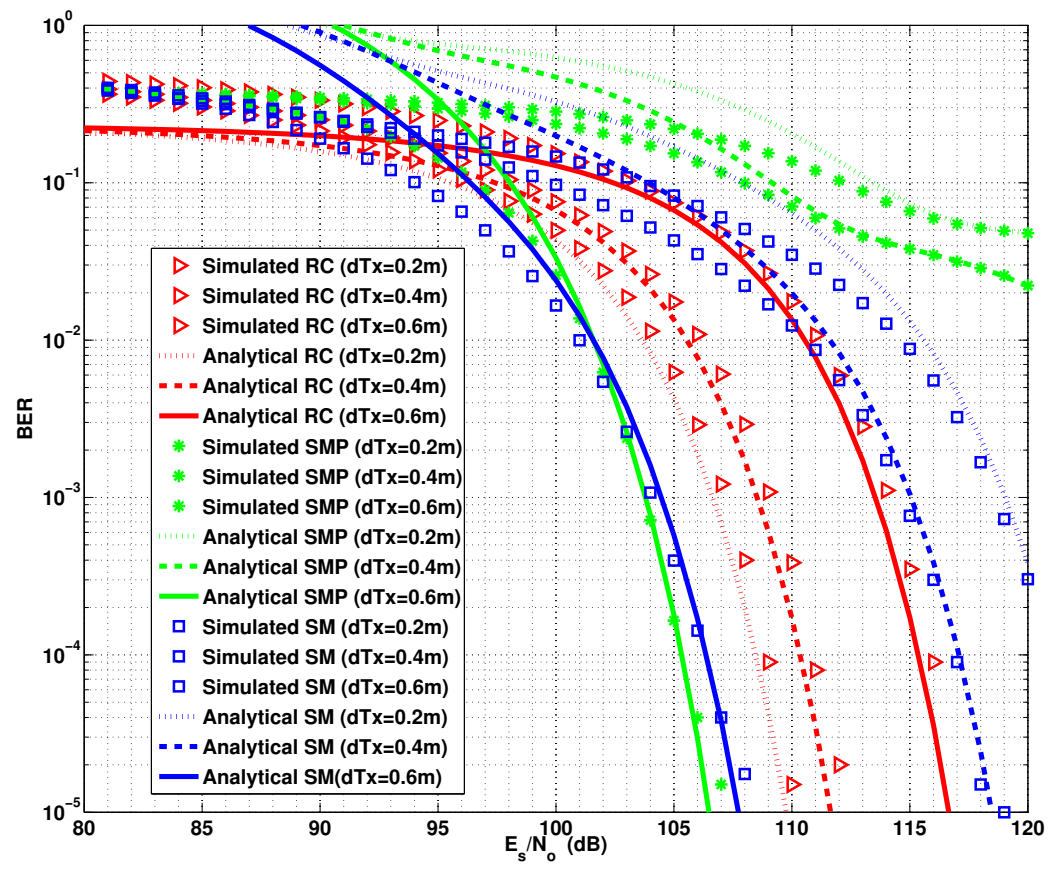

(a)

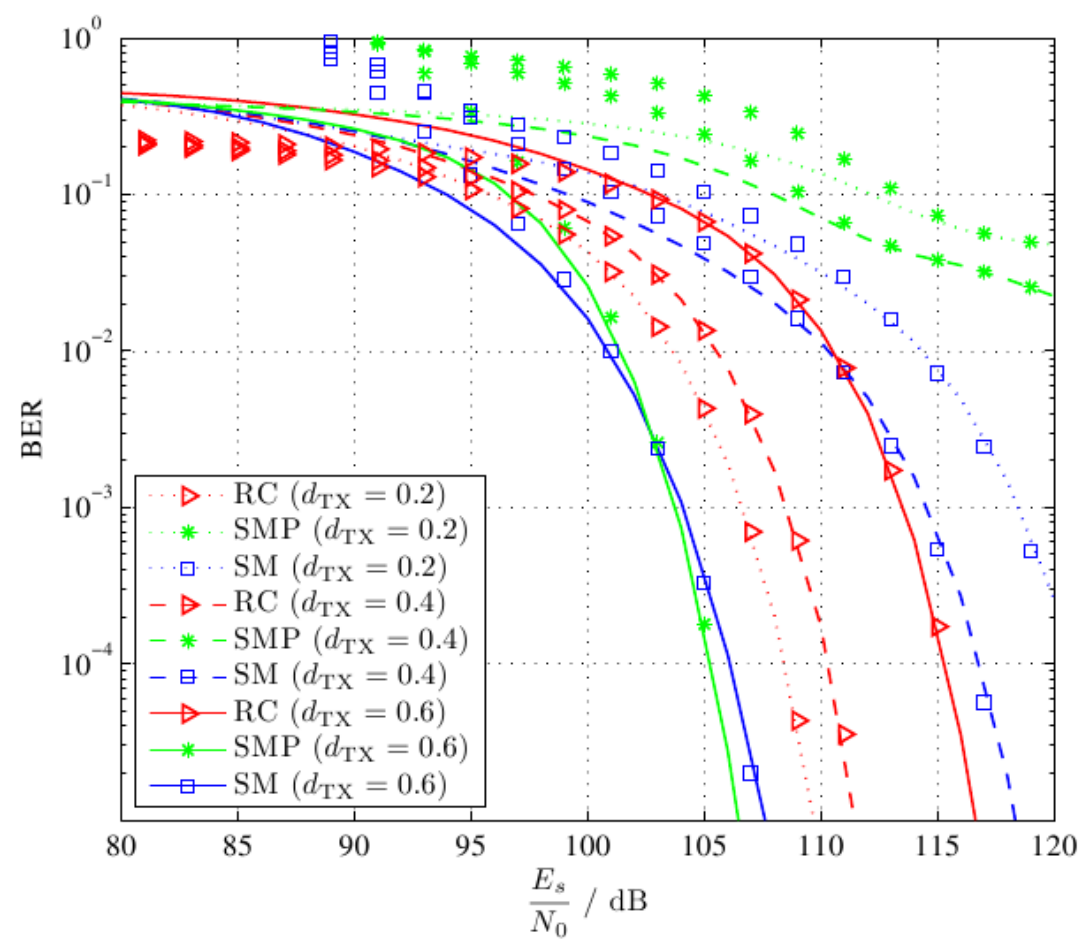

(b)

Figure 4.6: BER results (analytical and simulation) for MIMO vertical fixed detectors setup with TX separations of $0.2 \mathrm{~m}, 0.4 \mathrm{~m}$ and $0.6 \mathrm{~m}$ employing $R C$, SMP and SM (a) Simulated to compare with Figure 3 (a) of [47] (b) Copy of Figure 3 (a) of [47] 


\subsubsection{Angular diversity detectors setup}

The concept of orienting detectors in different directions is implemented, in order to improve the likelihood of LOS propagation between the TX and the RX and hence increasing spectral efficiency. The setup of TX and transmission techniques remains as in Figure 4.1 (a) but the receiver setup is modified as in Figure 4.1 (c). The coordinates $(x, y, z)$ of the detectors are the same for all of the four detectors but they are oriented at different elevation and azimuth angles. The azimuth separation between neighbouring sensors is $90^{\circ}$ to cover $360^{\circ}$ azimuth, and the elevation angle is varied from $45^{\circ}$ to $90^{\circ}$ as explained in section 4.2. The best angle is selected and the throughput is calculated for 1000 locations. The elevation angle of $75^{\circ}$ ( $15^{\circ}$ from vertical) for each detector was found to give a substantial capacity improvement, and angles greater or less than $15^{\circ}$ give relatively poor performance (Figure 4.7). Therefore elevation angle $15^{\circ}$ is used for comparison with the vertical receiver (Figure 4.10).

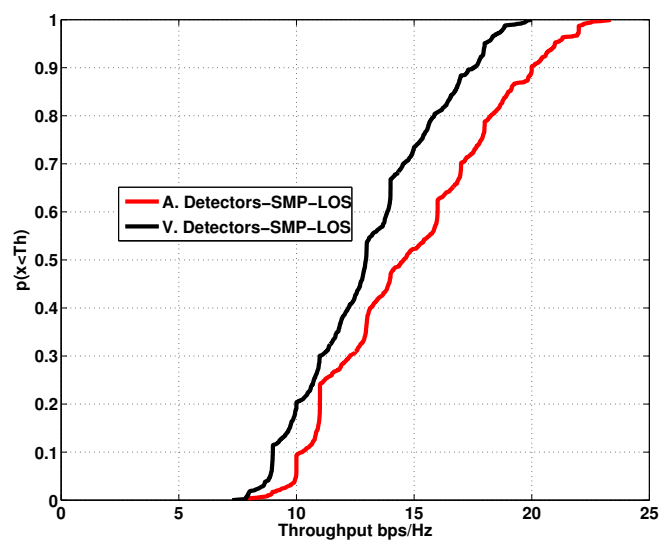

(a)

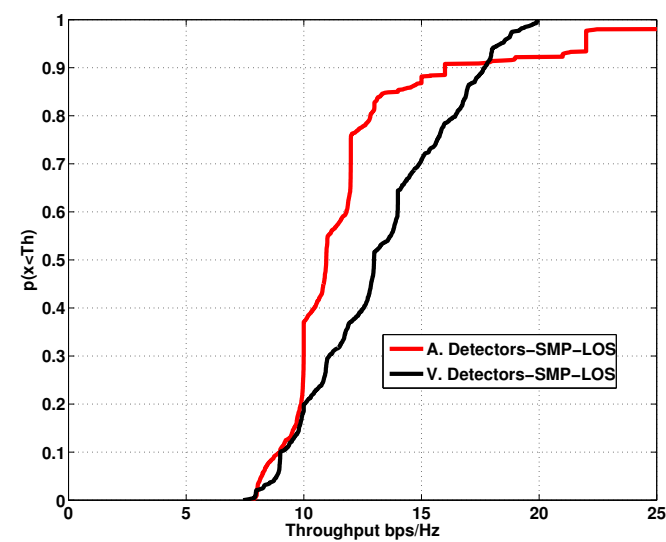

(b)

Figure 4.7: SMP CDF comparison between two elevation angles in angular diversity receiver detectors when LOS is considered (a) SMP, $10^{\circ}$ (b) SMP, $20^{\circ}$

\subsubsection{Comparison between Vertical detectors and Angular diversity detectors scenarios}

The performance of vertical detectors and angular diversity detectors for a mobile receiver is compared. All of the setups are simulated using all of the MIMO transmission techniques given in section 4.3. Figure 4.9 (a) shows the distribution of the channel matrix rank for the vertical detectors. It is shown that, the (60\%) of the room has rank 1, while in Figure 4.9 (b) 


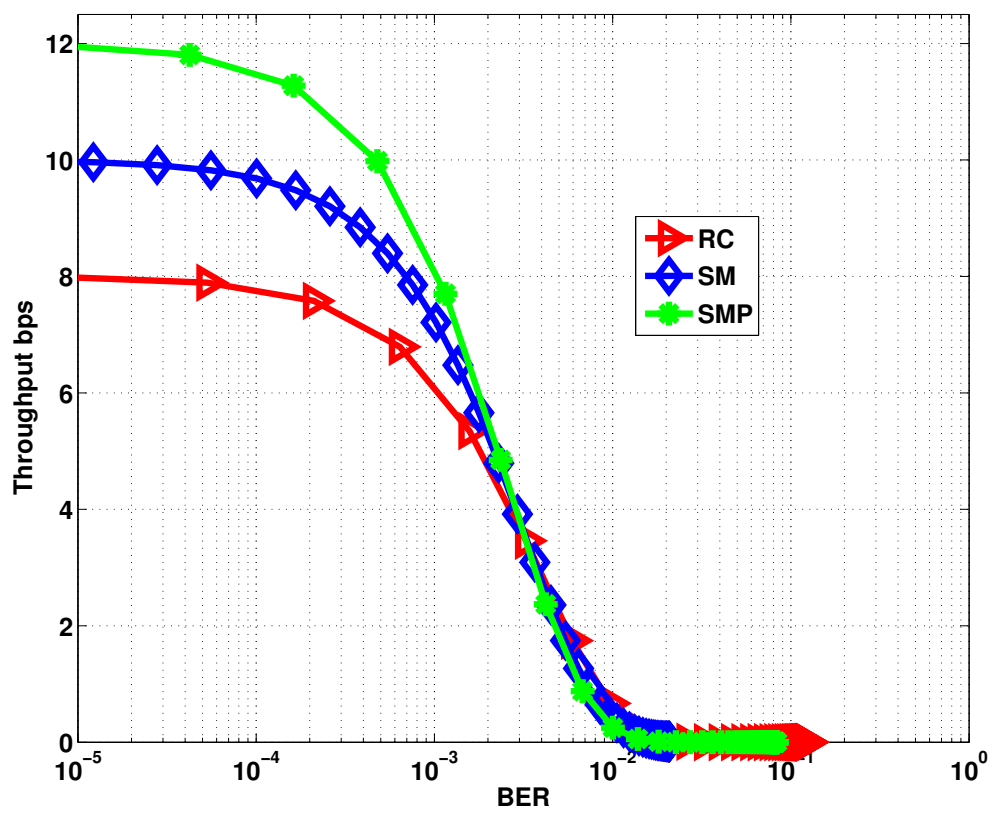

Figure 4.8: Throughput VS BER curves for SMP, SM and RC for angular diversity mobile receiver at $(2.05,1.95,0)$

\begin{tabular}{|c||c|c|c|c|c|c|}
\hline \multicolumn{1}{|c||}{ Trans- } & \multicolumn{5}{c|}{ Average throughput in bps/Hz } \\
\cline { 2 - 7 } \multirow{2}{*}{$\begin{array}{c}\text { mission } \\
\text { Method }\end{array}$} & \multicolumn{2}{|c|}{ V. Detectors $\left(0^{\mathbf{0}}\right)$} & \multicolumn{3}{c|}{ A. Detectors $\left(15^{\mathbf{0}}\right)$} \\
\cline { 2 - 7 } & $\mathbf{0 . 2} \mathbf{~ m}$ & $\mathbf{0 . 4} \mathbf{~ m}$ & $\mathbf{0 . 6} \mathbf{~ m}$ & $\mathbf{0 . 2} \mathbf{~ m}$ & $\mathbf{0 . 4} \mathbf{~ m}$ & $\mathbf{0 . 6} \mathbf{~ m}$ \\
\hline RC & 5.2 & 5.6 & 6 & 6.5 & 6.3 & 6.4 \\
\hline SM & 1.1 & 1.3 & 1.8 & 1.7 & 2.2 & 2.9 \\
\hline ASM & 5.2 & 5.8 & 7.6 & 6.1 & 7 & 10.1 \\
\hline SMP & 6 & 8.2 & 13.2 & 13 & 15.4 & 18.7 \\
\hline
\end{tabular}

Key to the table:

$\mathrm{V}=$ Vertically oriented, $\mathrm{A}=$ Angular diversity

$0.2 \mathrm{~m}$ to $\mathbf{0 . 6} \mathbf{~ m}$ are the transmitter separations

Table 4.2: Simulation results for LOS with different antenna separations

the proportion is reduced for the angular diversity detectors $(44 \%)$ indicating higher potential for MIMO with angular diversity receivers. Typical average throughput results for all setups (vertically oriented and angular diversity detectors) and their scenarios (TX separations) are in Table 4.2.

Results are shown for four techniques (RC, SM, ASM and SMP), with both V. Detector (vertically oriented detectors) and A. Detector (angular diversity detectors) configurations for three 


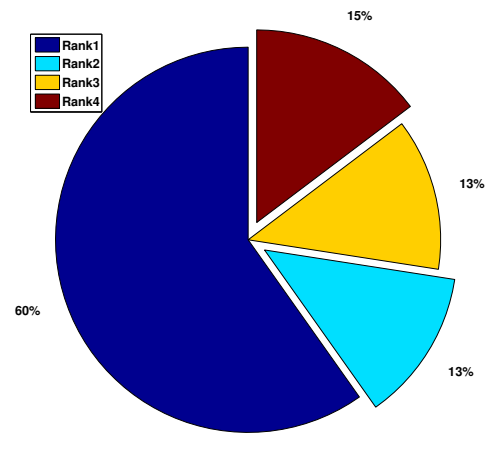

(a)

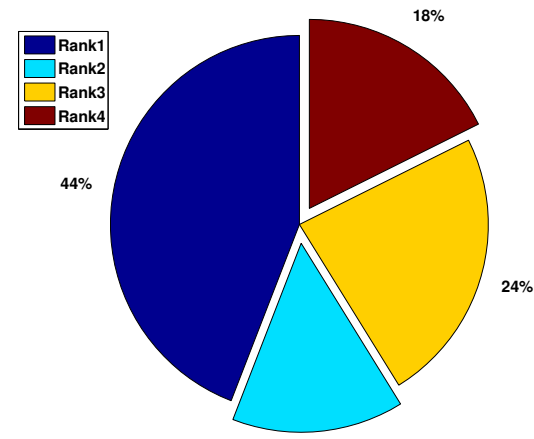

$15 \%$

Figure 4.9: The percentage distributions of MIMO channels matrix rank in 1000 locations of the room (a) Vertical detectors setup (b) Angular diversity detectors setup

transmitter spacings.

The $\mathrm{RC}$ results show that the average throughput for all setups does not vary significantly because the same data are transmitted for all the transmitting LEDs, so the same data rate is achieved whether the rank of channel matrix is 1 or 4 . Figure 4.10 (a) shows the CDF comparison between V. Detectors and A. Detectors in RC and the difference between two setups mainly relates to the coverage where the V. Detectors setup serves $10 \%$ fewer locations than the A. Detectors setup.

The SM results shows that the average throughput is worse because of the channel rank deficiency as explained in subsection 4.3.3. Figure 4.10 (c) shows that only $60 \%$ and $35 \%$ of the locations are covered for angular oriented detectors and vertical oriented detectors setups respectively. This poor performance is addressed in Adaptive Spatial Modulation.

The average throughput for ASM improves SM performance when the two receiver setups are simulated. It is shown that A. Detectors has $39.5 \%$ throughput improvement compared to V. Detectors for the same TX separation $(0.6 \mathrm{~m})$. This improvement is achieved because of changing transmission techniques and setups according to the channel matrix rank as explained earlier. In Figure 4.10 (d) the CDF results for ASM shows that the A. Detector achieves better coverage and hence throughput improvement. Figure 4.10 (d) shows that both setups cover $100 \%$ of the locations and that the A. Detectors shows better performance than the V. Detectors. The results of SM and ASM for a similar TX separation $(0.6 \mathrm{~cm})$ show that both setups have average throughput improvements greater than $100 \%$. 


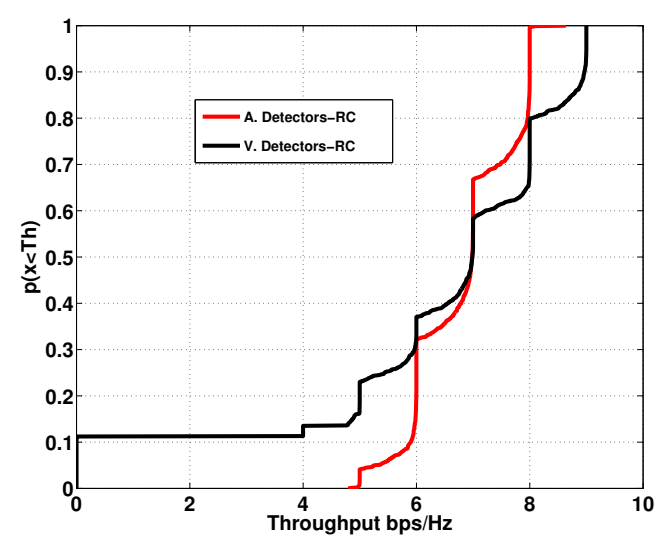

(a)

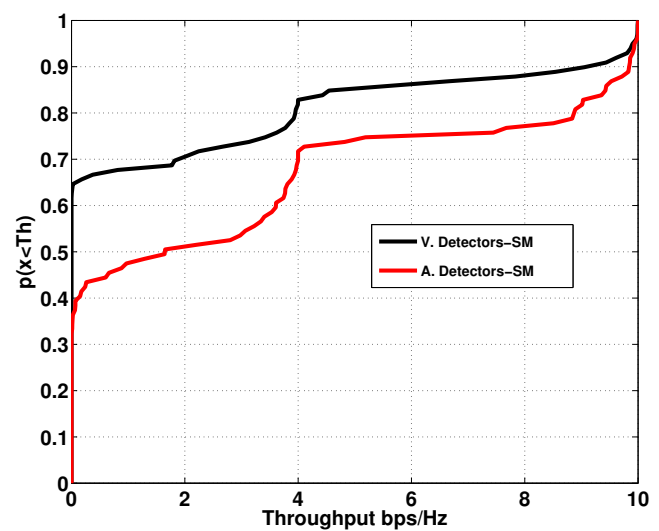

(c)

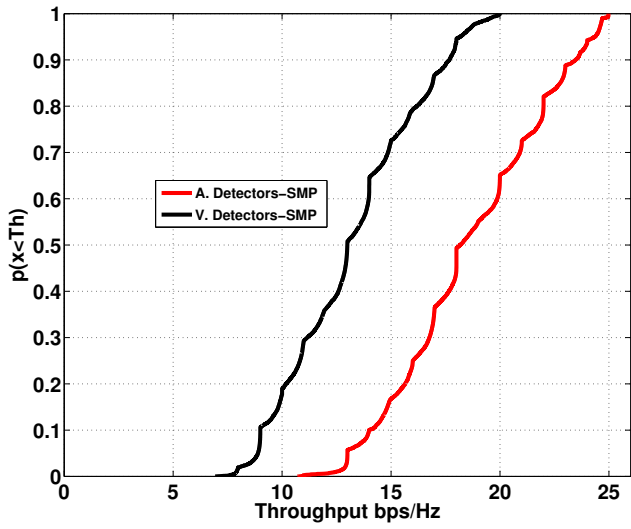

(b)

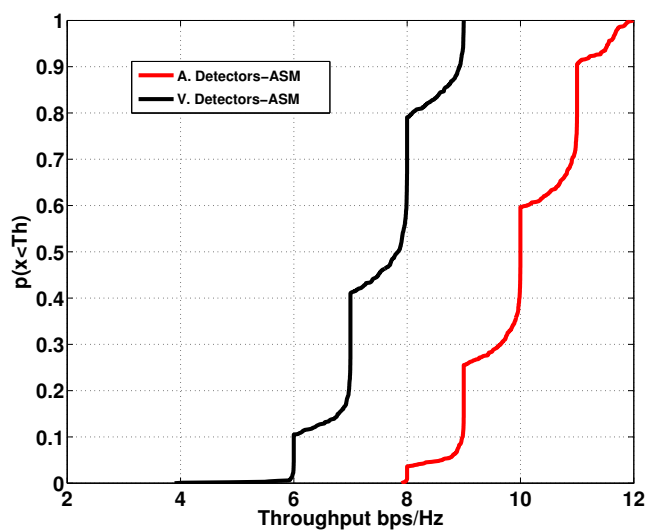

(d)

Figure 4.10: $C D F$ comparison between three MIMO techniques in vertically and angular diversity receiver detectors $(a) R C(b) S M P(c) S M(d) A S M$

SMP shows a significant throughput improvement compared to RC, SM and ASM. This is particularly true when compared with vertically oriented detectors; the channel rank for angular diversity detectors is increased to the higher likelihood of LOS propagation between TX and RX a gain in throughput is achieved. Note that increasing the TX separation decreases the channel correlation and gives significant improvement of throughput as transmitter separation is increased. For the same separation ( $0.6 \mathrm{~m})$, A. Detectors has a $42 \%$ throughput improvement compared to V. Detectors. Figure 4.10 (b) also shows that when SMP is used, there is a significant difference in throughput between the two setups, and for both cases, the coverage is $100 \%$. 


\subsection{Conclusion}

A mobile angular diversity optical receiver detector model in an indoor visible light communications is presented. Different MIMO transmission techniques with adaptive modulation and per antenna rate control are applied in order to evaluate the throughput across different room locations. Results for vertical detectors and angular diversity detectors setups in different scenarios are compared. Initial results show that using angular diversity detectors increase the likelihood of LOS among transmitters and receivers as shown in Table 4.2, Figure 4.9 and Figure 4.10. Also for a moving angular diversity receiver there is an improvement in throughput when using SMP with PARC (Table 4.2). It is shown that for the same setup, SMP has better performance compared to other candidates (RC, SM and ASM) as it is detailed in subsection 4.5.3. Percentage wise, for the same TX separation, SMP performs $120 \%$ and $192 \%$ better than RC for V. Detectors and A. Detectors respectively. Compared to ASM, SMP performs $78.9 \%$ and $85.1 \%$ better for V. Detectors and A. Detectors respectively. It was also seen that, using ASM where different TX/RX setups, transmission techniques and modulation are inter-changed, throughput improvements are achieved compared to always using SM with 4 transmitters. Generally the simulations suggest that, for mobile optical receivers, angular diversity detectors can perform better than vertical oriented receivers. Also ASM improves the SM performance in mobile receivers.

The next chapter will focus on the effect of reflected optical paths on conditions for the scenarios discussed in this chapter. The diffuse channels model provides further throughput improvement due to the fact that receivers can exploit both LOS paths and reflected paths. 


\section{Chapter 5 Performance analysis of indoor diffuse VLC MIMO channels using Angular Diversity Detectors}

In this chapter, specular and diffuse reflection models for indoor VLC using a mobile receiver with angular diversity detectors in MIMO channels are considered. The model is considered because the performance of the OWC LOS system may be jeopardized by obstructions. The target is to improve the MIMO throughput compared to vertically oriented detectors by exploiting reflections from different reflecting surfaces in the room. The throughputs across multiple locations in the small room by using repetition coding, spatial multiplexing and spatial modulation approaches is evaluated. The results for LOS only channels are compared with LOS with specular or diffuse reflection conditions, for both vertical and angular oriented receivers are then compared. These reflected optical paths have a positive impact on optical wireless channels because the receivers can exploit both the LOS paths and the reflected paths.

\subsection{Introduction}

Indoor OWC systems can be classified into diffuse and LOS systems. In LOS systems high data rates of the order of gigabits per second can be achieved $[1,13]$ but these systems are vulnerable to obstacles (shadowing) because of their directionality. In diffuse systems, several propagations paths exist from the LED to photo-diode (PD) which makes the system robust to shadowing [111], however the path loss can be higher than for LOS systems and multipath creates inter-symbol interference (ISI) for the case of large area indoor environments $[8,9,18$, 59]. The simultaneous use of multiple transmitters and receivers e.g. OWC multiple input multiple output (MIMO) can enhance the overall system performance and spectral efficiency as well as reducing the BER performance of a communication system [8]. MIMO systems can realize higher speed transmission without increasing the transmit power or the bandwidth. 
Kahn and Barry gives more details about wireless infrared communication in [41]. Here the use of infrared radiation as a medium for high-speed, short range OWC is discussed, and advantages and drawbacks are compared. MIMO techniques have been applied for OWC and data transmission to a limited extent. High data rate MIMO optical wireless communications using white LEDs were proposed in [8], where a fixed receiver with an imaging lens was used. It was shown that the imaging lens and detector array size are physically large and may not be practical for some applications. Another indoor OWC MIMO system with an imaging receiver was proposed in [112], here experiments were performed in a diffuse environment. The system shows error-free operation at $2 \mathrm{Mbit} / \mathrm{s} / \mathrm{spatial}$ channel at the center of the coverage area, with worse performance away from the center. In [94] results from several indoor OWC MIMO experiments were reported, a four channel MIMO system that uses white LEDs for communications was described as well as experiments in a diffuse environment using infra-red sources. An omnidirectional multibeam transmitter in [92] was proposed to improve transmission coverage and overcome shadowing, in this paper a multibeam hemispherical receiver structure was found to reduce multipath effects. The simulation results showed a significant reduction of the BER making the system suitable for high bit rate applications. An indoor optical wireless collaborative cellular system was proposed in [17]. In the paper, base stations and portable devices were designed, presented and analysed. Base stations (BS) providing multiple cells were proposed to cover a wide area, however the MIMO techniques did not perform properly at all of the receiver positions due to channel symmetry effects. Research development on overcoming channel correlation and inter-symbol interference yielded the method proposed in $[16,113,114]$. Here indoor OWC MIMO using spatial modulation (SM) was suggested and implemented, where unlike other MIMO techniques, only one transmitter is active at any given time instant. The active transmitter radiates at a certain intensity level and all other transmitters are turned off. In $\mathrm{SM}$, it was found that reducing both the distance between transmitters (TX) and receivers (RX) and the transmitter emerging angle (the angle between TX axis and the straight line to the RX) resulted in lower correlation and hence higher data throughput. A hemispherical lens based imaging receiver for OWC MIMO was described in $[115,116]$. These papers presented a novel imaging MIMO optical wireless system which uses a hemispherical lens in the receiver: this system has both a wide field of view (FOV) and showed significant spatial diversity. In [47], a performance comparison of OWC MIMO Techniques in indoor environments was provided between repetition coding (RC), SM and spatial multiplexing. The results show that SMP improves the spectral efficiency when there is low channel correlation. It was also shown that SM 
is competitive at low spectral efficiency while SMP performs better in high spectral efficiency where SM needs a very large signal constellation size to match SMP. Also SM is more robust to channel correlation. $\mathrm{RC}$ was found to be insensitive to different transmitter-receiver alignments but it needs a large signal constellation size to provide high data rates. The practical MIMO capacity for indoor optical wireless communication with white LEDs was presented in [117]. In this paper the communication scenario assumed an angle diversity receiver with multiple detector elements where each element has an independent concentrator/lens. A significant capacity improvement was achieved in such a system. In [97], the receiver performance improvement utilizing diversity in MIMO VLC with an imaging receiver was presented. It was shown that by employing maximal ratio combining at the receiver performance can be improved significantly, however the receiver may be impractical due to the size and complexity of imaging devices. T. Wang and J. Armstrong in $[118,119]$ analysed the performance of an indoor MIMO optical wireless system with a linear receiver. The receiver used an array of prisms to form channel matrices that can achieve angular diversity within a compact receiver structure. It was shown that full column rank can be achieved by the proposed receiver over an entire room. In [120] Wang and Chi experimentally demonstrated a $2 \times 2$ non-imaging MIMO VLC system that is capable to deliver $500 \mathrm{Mb} / \mathrm{s}$. However it was concluded that the large size of the lens and the detectors required are not practical. In [19], the receiver with angular diversity detectors was proposed and found to overcome the channel rank deficiency which occurs in areas away from the center of the room.

In these papers, VLC coverage is among the major drawbacks of the proposed systems. In most papers movement of the receiver to different locations to asses the impact on channel correlation and hence spectral efficiency was not covered. Further the effect of diffuse channel reflections on MIMO was not discussed. The present work addresses these issues and provides a novel study of the impact of specular and diffuse reflections on MIMO performance.

MIMO system taking into account LOS propagation is used, The Lambertian specular reflections model and the Lambert-Phong diffuse reflection model [111] for both vertical and angular oriented receiver detectors. The performance statistics for MIMO methods operating over many room locations using adaptive modulation and coding (AMC), ASM, and per antenna rate control (PARC) [102] is then provided. RX performance improvement by application of angular diversity techniques is also considered. 


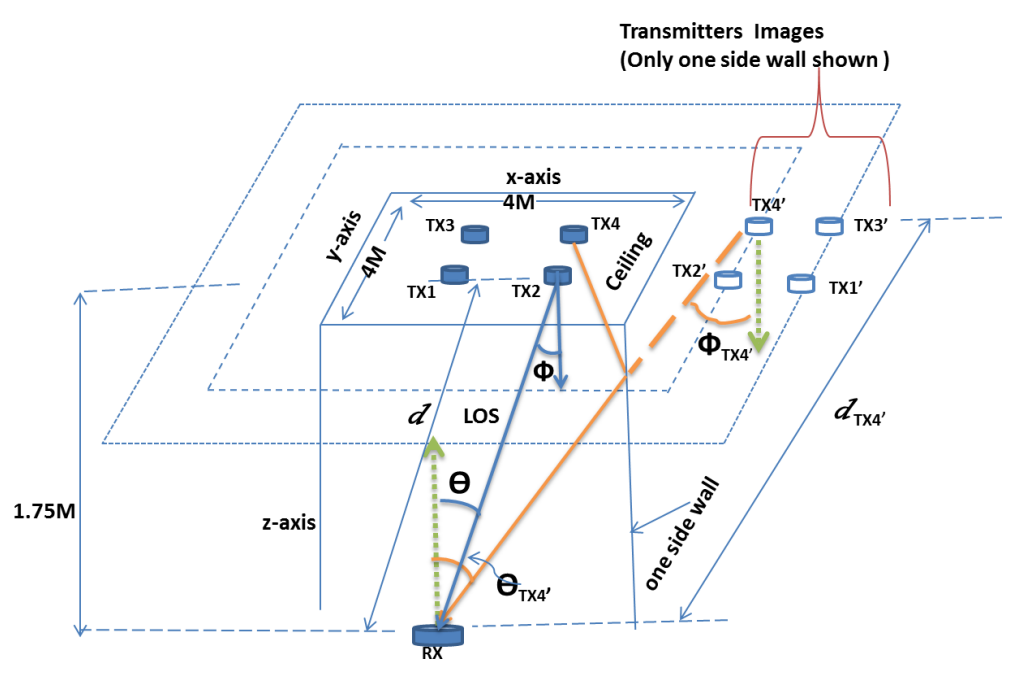

Figure 5.1: Transmitter and receiver geometry used for channel gain calculations in specular reflection model

\subsection{System models}

Visible light communications (VLC) MIMO transmission is considered taking into account both specular and diffuse reflections where intensity modulation (IM) and direct detection (DD) of the optical carrier using an incoherent light source is employed. The system consists of $N_{t}$ transmitters and $N_{r}$ photo-detectors at the receiver side. The size $N_{r}$ received signal vector $\mathbf{y}$ is

$$
\mathbf{y}=\mathbf{H} \mathbf{s}+\mathbf{n}
$$

Where $\mathbf{H}$ is the $N_{t} \times N_{r}$ channel matrix and $\mathbf{s}$ is the transmitted signal vector which is transmitted at a given time and is defined as follows: $\mathbf{s}=\left[s_{1} \ldots \ldots . s_{N t}\right]^{T}$ with [. $]^{T}$ being the transpose operator and $s_{n}$ denoting the signal transmitted by $n^{\text {th }}$ LED. The sum of the ambient light shot noise and thermal noise is denoted by $N_{r} \times 1$ vector $\mathbf{n}$ which is assumed to be real valued additive white Gaussian noise (AWGN) with zero mean and variance:

$$
\delta^{2}=\delta_{\mathrm{ss}}^{2}+\delta_{\mathrm{js}}^{2}
$$

Where $\delta_{\text {ss }}^{2}$ is the shot noise variance and $\delta^{2}{ }_{\mathrm{js}}$ is the thermal noise variance as calculated in 
equations (7) and (10) of [18] respectively. Thus the noise power is given by $\delta^{2}=N_{0} B$, where $N_{0}$ is the noise power spectral density and $B$ is communication bandwidth.

This chapter assumes an optical wireless LOS link operating in a room with reflection characteristics (Figure 5.1 and Figure 5.2) i.e the LOS and diffuse characteristics. There are two types of reflections considered in this chapter. The first type is a single bounce specular reflection which is modelled by an image transmitter $T X_{n}^{\prime}$ to the receiver (Figure 5.1). The reflections cause the signal to be attenuated by the surface reflection coefficient $\alpha$. The second type is a diffuse reflection where all the rays bouncing from the reflecting surface is scattered into different directions of the room (Figure 5.2 (a). The incidence power from the wall is assumed to be a fraction of the total transmitted power which is dictated by the number of rays re-radiated.

The LOS $N_{r} \times N_{t}$ channel matrix $\mathbf{H}$ for each room coordinate $(x, y, z)$ in the room is given by

$$
\mathbf{H}(x, y, z)_{L O S}=\left(\begin{array}{ccccc}
h_{11}(x, y, z) & h_{12}(x, y, z) & \cdot & \cdot & h_{1 N t}(x, y, z) \\
h_{21}(x, y, z) & h_{22}(x, y, z) & & \cdot \\
\cdot & & & \cdot & \cdot \\
\cdot & & & \cdot & \cdot \\
h_{N r 1}(x, y, z) & . & . & \cdot & h_{N r N t}(x, y, z)
\end{array}\right)
$$

where $h_{n_{r} n_{t}}$ represents the channel transfer function of the wireless link between transmitter $n_{t}$ and receiver $n_{r}$. For the specular reflection model (Sp) (Figure 5.1) and the diffuse reflection model ( $D f$ ) (Figure $5.2\left(\right.$ a)), the total channel gain at the receiver detector $n_{r}$ from transmitter $n_{t}$ is

$$
h_{n_{r} n_{t(S p / D f)}}=\sum_{i=1}^{N_{T X_{n^{\prime}}}} h_{n_{r} n_{t i}}
$$

where for $S p, N_{T X_{n}{ }^{\prime}}$ is the number of transmitter images received at detector $n_{r}$ caused by transmitter $n_{t}$ and for $D f, N_{T X_{n}{ }^{\prime}}$ is the number of scattered light rays received from transmitter $n_{t}$. Therefore, when all LOS, $S p$ and $D f$ rays are considered, the channel gain at the individual receiver detector $n_{r}$ will be

$$
h_{n_{r} n_{t(T o t a l)}}=h_{n_{r} n_{t(L O S)}}+h_{n_{r} n_{t(S p / D f)}}
$$

So, the overall $N_{r} \times N_{t}$ channel matrix $\mathbf{H}$ for each room coordinate $(x, y, z)$ in the room 
will be represented by substituting (5.5) into (5.3). The path difference between the multiple transmitter-receiver links is very small, on the order of few $\mathrm{cm}$ as shown in Figure 5.1. For simplicity it is assumed that the communications channel bandwidth is much less than the inverse of the delay spread, so the channel is not frequency selective.

By using the Lambert-Phong method [111] the diffuse paths are assumed to be scattered paths re-radiated from the wall to the receiver after being attenuated by the surface reflection coefficient $\alpha$. As illustrated in Figure 5.1, $\phi_{T X_{n}^{\prime}}^{\prime}$ is the angle of emergence with respect to the image of transmitter $T X_{n}$ axis, $\theta_{T X n}$ is the angle of incidence with respect to the receiver detector axis and $d_{T X_{n}^{\prime}}^{\prime}$ is the distance between the image transmitter $T X_{n}^{\prime}$ and receiver. Also as illustrated in Figure 5.2 (b), $\sigma$ is the angle of emergence with respect to the direction of specular reflected ray axis $\mathbf{f}$ which is a directional vector with coordinates $(x, y, z), \theta$ is the angle of incidence with respect to the receiver detector axis and $d$ is the distance between the reflecting point and the receiver. The system transmitters are fixed at the following ceiling coordinates: $T X_{1}[1.9 \mathrm{~m}, 1.9 \mathrm{~m}, 2.75 \mathrm{~m}], T X_{2}[2.5 \mathrm{~m}, 1.9 \mathrm{~m}, 2.75 \mathrm{~m}], T X_{3}[1.9 \mathrm{~m}, 2.5 \mathrm{~m}, 2.75 \mathrm{~m}]$, and $T X_{4}[2.5 \mathrm{~m}, 2.5 \mathrm{~m}, 2.75 \mathrm{~m}]$.

The transmitters are arranged in a square in the middle of the ceiling and the antenna side separation is $0.6 \mathrm{~m}$ as this choice was found to reduce channel correlation in [19].

Consider a LOS optical system propagation path in Figure 5.1, the channel gain from the transmitter to the receiver is given by [47]:

$$
h=\left\{\begin{array}{cc}
\frac{A(\kappa+1) \cos ^{\kappa}(\phi) \cos (\theta)}{2 \pi d^{2}}, & 0 \leq \theta \leq \varphi_{\frac{1}{2}} \\
0, & \theta>\varphi_{\frac{1}{2}}
\end{array}\right.
$$

where $\kappa=\frac{-\ln (2)}{\ln \left(\cos \left(\Phi_{1 / 2}\right)\right)}$.

The scalar $A$ is the collection area of the receiver $n_{r}, \Phi_{1 / 2}$ is the transmitter semi-angle (at half power), which is assumed to be $45^{\circ}$. The scalar $\varphi_{1 / 2}$ is the Field of View (FOV) semi angle of the receiver which is also assumed to be $45^{\circ}$. In [47] $\Phi_{1 / 2}$ and $\varphi_{1 / 2}$ were assumed to be both $15^{\circ}$, but for this setup where the receiver is moving around the room, it is difficult to achieve LOS channel conditions in many locations when a narrower half angle is used. 


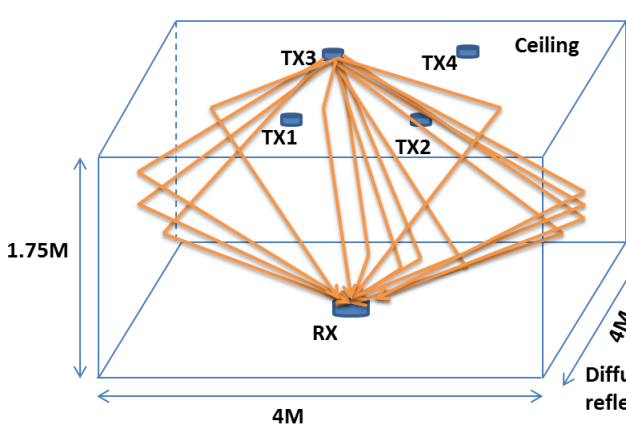

(a)

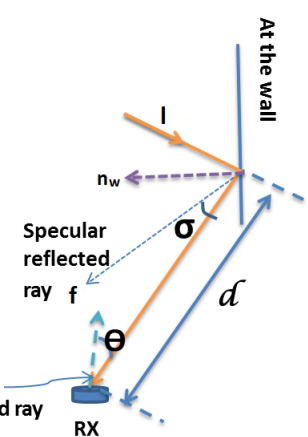

(b)

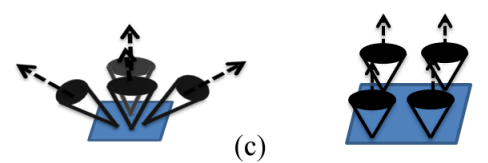

(c)

Figure 5.2: Transmitter and receiver geometry used for channel gain calculations (a) Diffuse reflection model (b) Diffuse reflection-wall geometry (c) Receiver with inclined and vertical axis detectors

When a receiver and transmitter are not in each others' FOV, $h_{n_{r} n_{t(L O S)}}=0$. Image transmitters in the specular reflection model use the same equation (5.6) with their respective angles, then the gain is multiplied by the reflection attenuation $\alpha$ to yield the corresponding channel gain

$$
h=\alpha \frac{A(\kappa+1)}{2 \pi d_{T X_{n}^{\prime}}{ }^{2}} \cos ^{\kappa}\left(\phi_{T X_{n}^{\prime}}\right) \cos \left(\theta_{T X_{n}^{\prime}}\right)
$$

For the diffuse reflection case the Lambert-Phong model is used [111]. A reflection intensity scattering is defined using a generalized Lambert radiation pattern

$$
P_{w}=\alpha \frac{P_{i}\left(m_{s}+1\right)}{2 \pi} \cos ^{m_{s}}(\sigma)
$$

where $P_{i}$ is the incident normalized unit power arriving at the wall, $P_{w}$ is the reflection intensity from the reflecting surface, $m_{s}$ is the smoothness of the reflecting material and $\sigma$ is the randomly generated parameter which represents the angle between specular reflected rays and the diffuse reflected ray (Figure 5.2 (b). To calculate the specular reflected ray vector $\mathbf{f}$ the normal vector $\mathbf{n}_{\mathbf{w}}$ to the wall and the vector $\mathbf{l}$ (which is the line connecting transmitter and the wall) are used (see Figure 5.2 (b)): 


$$
\mathbf{f}=\left(\mathbf{2} \mathbf{n}_{\mathrm{w}} \bullet \mathbf{l}\right) \mathbf{n}_{\mathrm{w}}-\mathbf{l}
$$

where $\mathbf{f}, \mathbf{n}_{\mathbf{w}}$ and $\mathbf{l}$ are three dimensional vectors with components (x, y, z). The dot mark in (5.9) denotes the vector dot product. As shown in Figure $5.2(\mathrm{~b})$, once $\mathbf{f}$ and $\sigma$ are known then all the diffusely reflected paths can be generated. So, the channel gain for one reflected ray from the wall to the receiver is expressed as

$$
h\left(\sigma, m_{s}\right)=\left\{\begin{array}{cc}
\alpha \frac{A\left(m_{s}+1\right)}{2 \pi d^{2}} \cos ^{m_{s}}(\sigma) \cos (\theta), & 0 \leq \theta \leq \varphi_{1 / 2} \\
0, & \theta>\varphi_{1 / 2}
\end{array}\right.
$$

and the incident optical power to the receiver, see $[18,89,103]$ can be calculated by

$$
P_{r}=P_{w} \frac{A \cos (\theta)}{d^{2}}=h\left(\sigma, m_{s}\right) P_{i}
$$

The technique used for configuring the inclined optical detectors of the receiver (the left side of Figure 5.2 (c)) is detailed in section 4.2 and equation 4.5.

\subsection{Review of the MIMO techniques}

In this chapter the same four MIMO techniques are used as in chapter 4; RC, SM, ASM and SMP. In contrast to $[8,16,94]$ where fixed receivers are used, in this thesis it is considered that mobile receiver is able to move freely around the room and to overcome the resulting SNR variations adaptive modulation with per antenna rate control (PARC) [102] in SMP and adaptive modulation in SM and RC are employed. In these techniques, the modulation $M$-level is chosen and updated in each transmit time and at each location depending on the current channel conditions. It is again assumed that all considered MIMO techniques use maximum likelihood (ML) [47] detection at the receiver with perfect knowledge of the channel and ideal time synchronisation except for SMP where the zero forcing (ZF) detection method is used to reduce receiver complexity when using PARC.

In RC the same signal is simultaneously emitted from all transmitters and the light intensities arising from the several transmitters constructively add up at the receiver side. In SM the conventional constellation diagram is extended to an additional dimension namely, the spatial 
dimension. Specifically, the LED index is used to communicate data bits to the receiver. Each transmitting LED is assigned a unique binary sequence (the spatial symbol). ASM is a modified form of SM designed to cope with rank deficient channels due to the fact that, when the receiver moves around the room the channel matrix $\mathbf{H}$ is not full rank i.e rank (4) in many locations. If the channel matrix rank is reduced, the receiver can not easily distinguish all the transmitting LEDs. By using antenna selection techniques, the ASM receiver checks the rank of the channel matrix and decides which TX/RX setup is to be used. In SMP a source stream is split into several substreams and each substream is then transmitted from a different transmitter. In this case, all the transmitters are active at the same time and each receiver uses multiple antennas to detect the multiple co-channel signals. The above mentioned MIMO techniques are desribed in detail in Chapter 4, section 4.3. In this chapter the system throughput evaluation methods detailed in chapter 4 , section 4.4 are used.

\subsection{Simulation parameters and results}

A $4 \times 4$ indoor MIMO scenario as in [19] and in chapter 4 is considered, but in this chapter the effect of wall reflections is also considered. The system is located within a room of size $4 \times 4 \times 3 \mathrm{~m}$ and it is assumed that the transmitters are placed at a height of $2.50 \mathrm{~m}$ and oriented downwards perpendicular to the floor of the room. The receiver is allowed to move randomly at a height of $0.75 \mathrm{~m}$ (human waist or table height) and its detectors are either placed vertically or oriented at a given elevation angle as in Figure 5.2 (c). The inclined detector's orientation is meant to increase the likelihood of FOV overlap between the transmitters and the receivers and also incoming reflected rays to the receiver, thus increasing system spectral efficiency.

A computer program that implements the scenarios presented in previous sections was written using MATLAB software. The simulation parameters are tabulated in Table 5.1. The room size shows the dimension of the room where the simulation is assumed. The TX/RX setup defines the number of transmitters and receivers used in simulation. The TX separation shows how far the four transmitting LEDs are separated from each other, the RX separation shows how the receiver detectors are separated (this applies for the vertically oriented receiver). The TX/RX FOV are the Field of View angles of the transmitter and receiver respectively. The RX detectors elevation angle is the one between detector's axis and the horizontal. For angular oriented detectors (A. detectors) this angle is varied to determine the optimum one while for the vertically oriented detectors ( $\mathrm{V}$. detectors) the angle of elevation is always $90^{\circ}$ from the 
Performance analysis of indoor diffuse VLC MIMO channels using Angular Diversity

\begin{tabular}{|c||c|}
\hline \multicolumn{1}{|c||}{ Parameters } & Values \\
\hline Room size $(W \times L \times H)$ & $4 \mathrm{~m} \times 4 \mathrm{~m} \times 3 \mathrm{~m}$ \\
\hline Number of TX/RX & $4 \times 4$ \\
\hline TX separation & $0.6 \mathrm{~m}$ \\
\hline Reflection coefficients $(\alpha)$ & $0.1,0.3,0.5,0.7,0.9$ \\
\hline Reflection parameter $\left(m_{s}\right)$ & 1 (rough surface) \\
\hline RX separations (V. detectors) & $0.1 \mathrm{~m}$ \\
\hline Photodiode responsivity $(\varrho)$ & $1 \mathrm{~A} / \mathrm{W}$ \\
\hline RX random rotation angles & $0^{\circ}$ to $360^{\circ}$ \\
\hline TX/RX FOV & $45^{\circ}, 45^{\circ}$ \\
\hline RX elevation angle (A. Diversity) & $45^{\circ}$ to $90^{\circ}$ towards vertical \\
\hline RX azimuth angle separation $($ A. Diversity) & $90^{\circ}$ \\
\hline Photodiode area $(A)$ & $1 \mathrm{~cm}{ }^{2}$ \\
\hline MIMO transmission techniques & RC, SM, ASM, SMP \\
\hline Modulation schemes & 2 PAM-1024PAM \\
\hline
\end{tabular}

Table 5.1: Parameters used for simulation

horizontal plane. The RX detectors azimuth angle separation shows how four detectors are angularly separated around $360^{\circ}$ for the angular diversity detectors setup (A. detectors). It is assumed that users hold their devices in a random orientation in the range of $0^{\circ}-360^{\circ}$ azimuth angles from the position of the detector. This is achieved by applying equation (4.5) from chapter 4 to all the detector locations. The experiment set-up and system performance comparison are presented in the next subsections:

\subsubsection{Setting up Vertical detectors}

To validate our results, the setup developed in [47] was repeated for all proposed MIMO transmission techniques and different TX separations $(0.2 \mathrm{~m}, 0.4 \mathrm{~m}, 0.6 \mathrm{~m})$. The RX detector separation remains $0.1 \mathrm{~m}$ all the time with a vertically oriented $\mathrm{RX}$ as in Figure 5.2 (c) right.

Mobile receiver model is then developed and evaluated the throughput for 1000 locations. In this case (of a mobile receiver) adaptive modulation is imployed for all transmission techniques, i.e. the rate of transmission is updated at each room location depending on the channel conditions. In addition to adaptive modulation PARC is considered in SMP. The results for average throughputs of the different transmission techniques and different reflection coefficients are compared in Table 5.3 and 5.4. 
Performance analysis of indoor diffuse VLC MIMO channels using Angular Diversity

Detectors

Further, the CDF for all techniques are shown in Figure 5.5, 5.6, 5.7 and 5.8. Both data tables and CDF plots are explained in subsection 5.4.2.

\begin{tabular}{|c||c|c|c|c|c|c|}
\hline \multicolumn{1}{|c||}{\multirow{2}{*}{$\begin{array}{c}\text { Trans- } \\
\text { mission } \\
\text { Method }\end{array}$}} & \multicolumn{4}{c|}{ Average throughput in bps/Hz } \\
\cline { 2 - 7 } & \multicolumn{2}{|c|}{ V. detectors $\left(0^{\mathbf{0}}\right)$} & \multicolumn{3}{c|}{ A. detectors $\left(15^{\mathbf{0}}\right)$} \\
\cline { 2 - 7 } $\mathbf{\text { RC }}$ & 5.2 & 5.6 & 6 & 6.5 & 6.3 & 6.4 \\
\hline SM & 1.1 & 1.3 & 1.8 & 1.7 & 2.2 & 2.9 \\
\hline ASM & 5.2 & 5.8 & 7.6 & 6.1 & 7 & 10.1 \\
\hline SMP & 6 & 8.2 & 13.2 & 13 & 15.4 & 18.7 \\
\hline
\end{tabular}

Key to the table:

$\mathrm{V}=$ Vertically oriented, $\mathrm{A}=$ Angular diversity

$0.2 \mathbf{~ m}$ to $0.6 \mathrm{~m}$ are the transmitter separations

Table 5.2: Simulation results for LOS with different antenna separations [from Table 4.2]

\begin{tabular}{|c|c|c|c|c|c|c|c|c|c|c|c|c|}
\hline \multirow{3}{*}{$\begin{array}{l}\text { Trans- } \\
\text { mission } \\
\text { Method }\end{array}$} & \multicolumn{12}{|c|}{ Average throughput in bps/Hz } \\
\hline & \multicolumn{6}{|c|}{ V. detectors $\left(0^{\circ}\right)$} & \multicolumn{6}{|c|}{ A. detectors $\left(15^{\circ}\right)$} \\
\hline & LOS & 0.1 & 0.3 & 0.5 & 0.7 & 0.9 & LOS & 0.1 & 0.3 & 0.5 & 0.7 & 0.9 \\
\hline $\mathbf{R C}$ & 6.0 & 6.0 & 6.4 & 7.0 & 7.1 & 7.3 & 6.4 & 6.9 & 7.0 & 7.1 & 7.2 & 7.3 \\
\hline SM & 1.8 & 1. & 1.9 & 2.2 & 3. & 4 & 2. & 3. & 3.9 & 4.2 & 6.3 & 7.0 \\
\hline ASM & 7.5 & 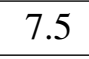 & 8.6 & 8 & 9.1 & 9.4 & 10.0 & 10.1 & 10.3 & 10.3 & 10.4 & 10.5 \\
\hline SMP & 13.1 & 13.3 & 13.7 & 14.0 & 14.3 & 14.6 & 18.6 & 18.8 & 19.1 & 19.5 & 20.0 & 20.4 \\
\hline
\end{tabular}

Key to the table:

$\mathrm{V}=$ Vertically oriented, $\mathrm{A}=$ Angular diversity

Parameters 0.1 to 0.9 are the reflection coefficients $(\boldsymbol{\alpha})$ of the reflecting surface

Table 5.3: Simulation results for LOS with Specular reflections

\begin{tabular}{|c|c|c|c|c|c|c|c|c|c|c|c|c|}
\hline \multirow{3}{*}{$\begin{array}{l}\text { Trans- } \\
\text { mission } \\
\text { Method }\end{array}$} & \multicolumn{12}{|c|}{ Average throughput in bps/Hz } \\
\hline & \multicolumn{6}{|c|}{ V. detectors $\left(0^{\circ}\right)$} & \multicolumn{6}{|c|}{ A. detectors $\left(15^{\circ}\right)$} \\
\hline & LOS & 0.1 & 0.3 & 0.5 & 0.7 & 0.9 & $\mathrm{LOS}$ & 0.1 & 0.3 & 0.5 & 0.7 & 0.9 \\
\hline $\mathbf{R C}$ & 6.0 & 6.2 & 9.6 & 10.4 & 10.9 & 11.1 & 6.4 & 6.8 & 10.3 & 10.8 & 11.3 & 11.5 \\
\hline SM & 1.8 & 3.5 & 4.8 & 5.1 & 6.0 & 6 & 2.9 & 4.9 & 6.1 & 6.4 & 6.9 & 7.6 \\
\hline ASM & 7.5 & 7. & 13.2 & 13.7 & 14.3 & 14.6 & 10.0 & 14.6 & 16.9 & 17.6 & 18.0 & 18.4 \\
\hline SMP & 13.1 & 13.1 & 16.5 & 19.7 & 20.8 & 21.6 & 18.6 & 22.4 & 26.2 & 29.2 & 30.4 & 31.6 \\
\hline
\end{tabular}

Key to the table:

$\mathrm{V}=$ Vertically oriented, $\mathrm{A}=$ Angular diversity

Parameters 0.1 to 0.9 are the reflection coefficients $(\alpha)$ of the reflecting surface

Table 5.4: Simulation results for LOS with diffuse reflections 


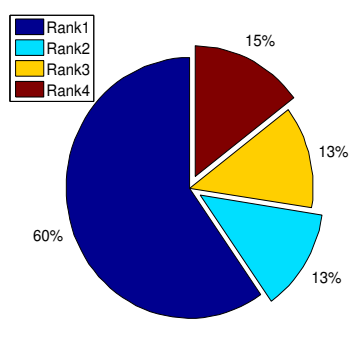

(a) V. detectors LOS

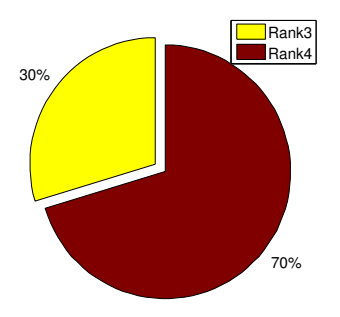

(d) A. detectors $(\alpha=$

$0.3)$

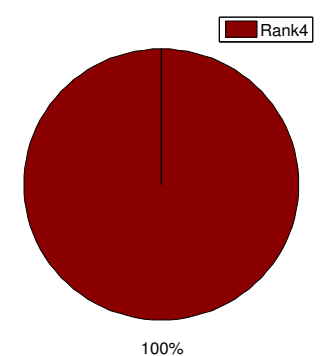

(g) V. detectors

( $\alpha=$ either 0.3 or 0.5 )

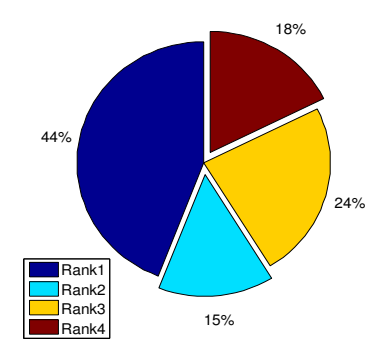

(c) V. detectors $(\alpha=$

(b) A. detectors (LOS)

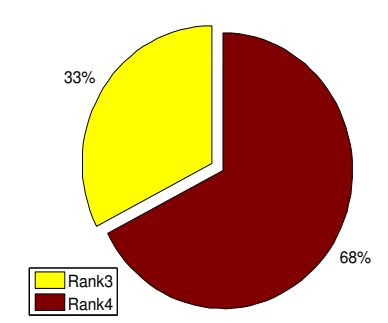

(f) A. detectors $(\alpha=$

(e) V. detectors $(\alpha=0.5)$

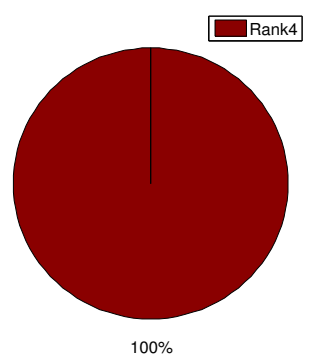

(h) A. detectors

( $\alpha=$ either 0.3 or 0.5 )

Figure 5.3: Percentage distributions of MIMO channels matrix rank in 1000 locations of the room (a) Vertical detectors setup (b) Angular diversity detectors setup (c \& e) Vertical detector setup with specular reflections ( $d \& f)$ Angular diversity detectors setup with specular reflections $(g \& h)$ Both vertical and angular detectors setups with diffuse reflections 


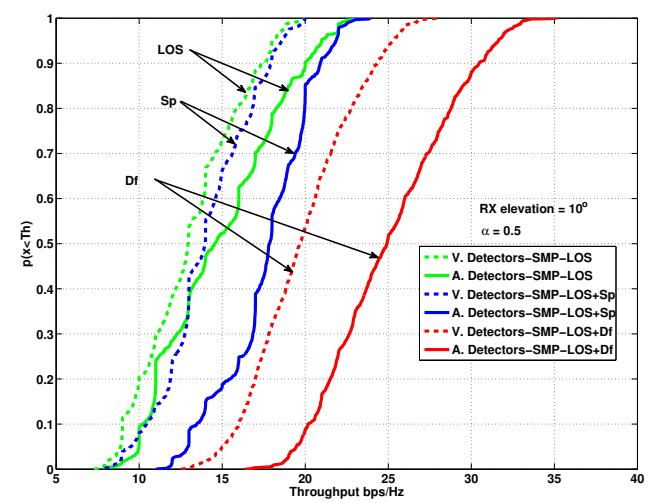

(a)

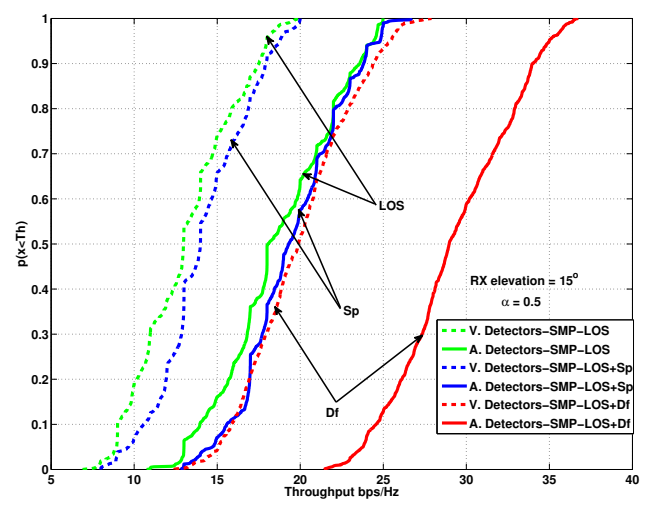

(b)

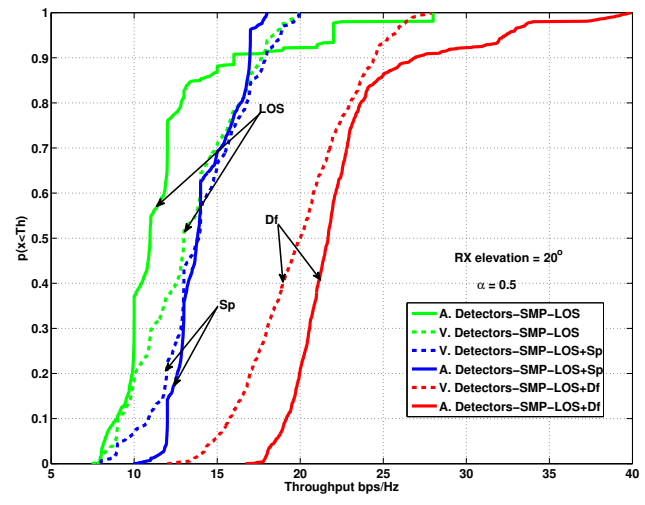

(c)

Figure 5.4: SMP CDF comparison between three elevation angles in angular diversity receiver detectors when LOS, LOS $+D f$ and LOS + Sp are considered, $\alpha=0.5$ (a) $S M P, 10^{\circ}$ (b) SMP, $15^{\circ}$ (c) SMP, $20^{\circ}$ 


\subsubsection{Comparison between Vertical detectors and Angular diversity detectors scenarios with specular and diffuse reflections.}

Here the performance of vertical detectors and angular diversity detectors for a mobile receiver is compared taking into consideration the effects of wall reflections and receiver. All the setups were simulated using all the MIMO transmission techniques discussed in section III. Using the throughput calculations as explained in section 4.4, typical average throughput results for all setups (vertically oriented and angular diversity detectors) and their scenarios (reflection types and reflection coefficients) are tabulated in Table 5.3 and 5.4. Results are shown for four techniques (RC, SM, ASM and SMP), with both V. detectors (Vertically oriented detectors) and A. detectors (angular diversity detectors). Figure 5.4. shows the SMP CDF comparison for three different elevation angles in angular diversity receiver $\left(10^{\circ}, 15^{\circ}\right.$ and $\left.20^{\circ}\right)$ when $L O S$, $L O S+D f$ and $L O S+S p$ are considered. Looking at the A. detectors $(L O S+D f)$ results on the same figure it is shown that elevation angles of $10^{\circ}, 15^{\circ}$ and $20^{\circ}$ give throughputs of 24.8 $\mathrm{bps} / \mathrm{Hz}, 29.2 \mathrm{bps} / \mathrm{Hz}$ and $22.3 \mathrm{bps} / \mathrm{Hz}$ respectively and therefore $15^{\circ}$ is used in all the subsequent simulation results. Figure 5.5. shows the RC CDF comparison between V. detectors and A. detectors with and without reflections, also with different reflection coefficients. For simplicity only the CDF results for reflection coefficients of $0.3,0.5$, and 0.7 are plotted for all the curves, other results are recorded in Tables 5.3 and 5.4.

\subsubsection{Result for LOS channel}

Table 5.2 shows results for different antenna separations matching to [19] where the four techniques (RC, SM, ASM, SMP) in LOS were compared in detail. Looking at the table it can be seen that, for a moving angular diversity receiver there is an improvement in throughput when using SMP with PARC compared to other techniques (RC, SM and ASM). Percentage wise, for a TX separation of $0.4 \mathrm{~m}$, SMP performs $46 \%$ and $144 \%$ better than RC for V. Detectors and A. Detectors respectively. In comparison to ASM, SMP performs $41 \%$ and $120 \%$ better for V. Detectors and A. Detectors respectively. For a TX separation of $0.6 \mathrm{~m}$, SMP performs $120 \%$ and $192 \%$ better than RC for V. Detectors and A. Detectors respectively and when compared to ASM, SMP performs $78.9 \%$ and $85.1 \%$ better for V. Detectors and A. Detectors respectively. All the subsequent results in this chapter are for $0.6 \mathrm{~m}$ transmitter spacing as this spacing provides the best performance in Table 5.2. Here, the channel rank of different receiver locations in the room is also considered. 


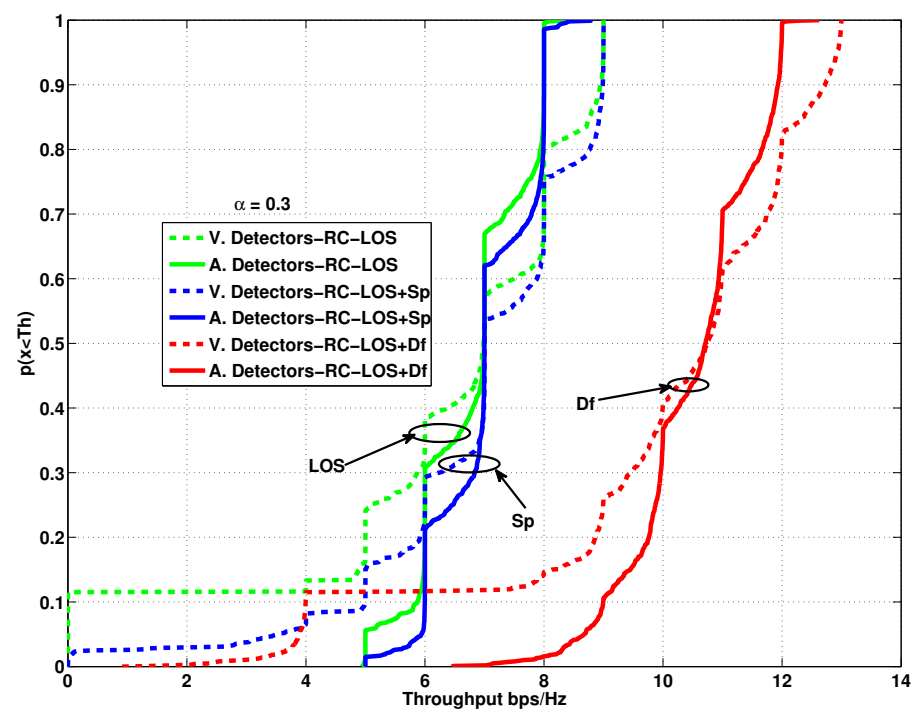

(a)

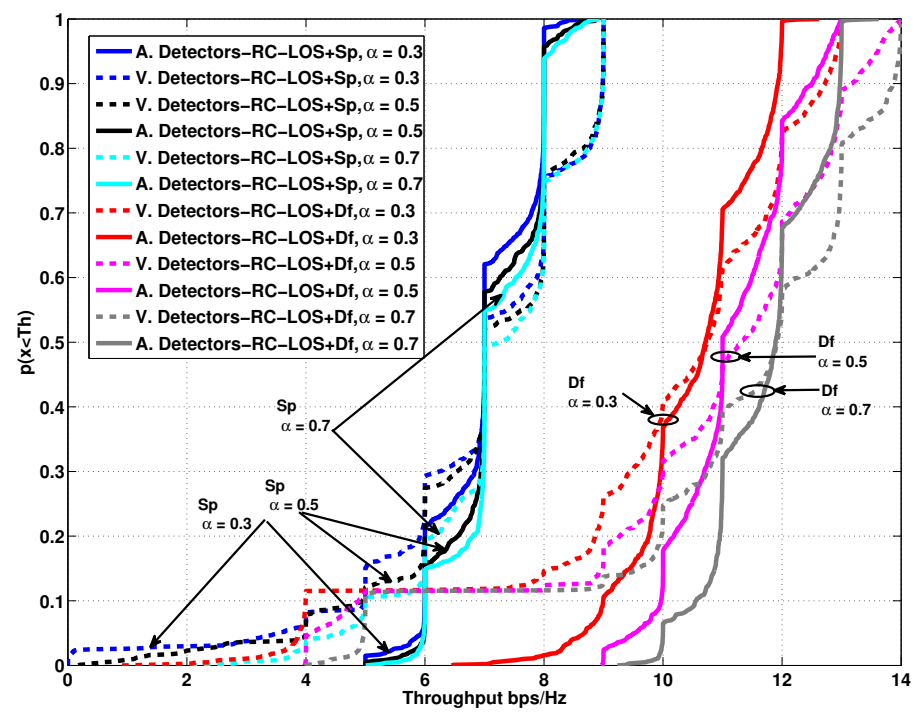

(b)

Figure 5.5: $R C C D F$ comparison between three reflection coefficients in vertically and angular diversity receiver detectors when $L O S, L O S+S p$ and $L O S+D f$ are considered (a) LOS Vs LOS +Sp, $\alpha=0.3$ (b) LOS +Sp Vs LOS + Df, $\alpha=0.3,0.5$ and 0.7 . 
Looking at Figure 5.3 (a) it is shown that the channel matrix rank for the vertical detector is 1 for a large area of the room (60\%) while in Figure 5.3 (b) the proportion is reduced for the angular diversity detectors (44\%) which indicates a higher potential for MIMO receivers. Figure 5.5 (a). shows that the difference between the two setups in LOS conditions mainly relates to the coverage where $10 \%$ of the $\mathrm{V}$. detectors setup give zero throughput to the total loss of paths between TX and RX. The A. detectors setup gives non zero throughput results in all room locations.

\subsubsection{Results for LOS with specular reflection channel}

The effects of reflections are clearly seen when looking at Figure 5.3 (c) through (h). In Figure 5.3 (c) when specular reflection with a reflection coefficient $\alpha=0.3$ is considered in V. detectors, the proportion of rank 1 channel reduces to $27 \%$ and rank 2 increases to $55 \%$. In Figure 5.3 (d) when specular reflections $(\alpha=0.3)$ are included in the A. detectors setup, the channel matrix rank values are either only $3(30 \%)$ and $4(70 \%)$ as the chance of multipath reception has increased. Also in Figure 5.3 (e) when specular reflections $(\alpha=0.5)$ are included in the V. detectors setup, the channel matrix rank is either $3(33 \%)$ or $4(68 \%)$ as the chance of multipath reception has further increased compared to V. detectors $(\alpha=0.3)$. Figure 5.3 (f) shows the effect of specular reflections on A. detectors $(\alpha=0.5)$, the rank 4 case dominates $(90 \%)$ and only $10 \%$ of channels have rank 3 .

Looking at Table 5.3, the $\mathrm{RC}$ results show that the average throughput for all specular reflection coefficients does not vary significantly because the same data are transmitted by all transmitting LEDs so whether the rank of channel matrix is 1 or 4 , the same data rate is achieved. The specular reflection results in Figure 5.5 shows that both detectors setups give non zero throughput because of the gain caused by the specular reflected rays. Also both detector setups have negligible difference in their throughput performance because RC cannot exploit higher MIMO channel rank to increase throughput.

Table 5.3 shows the average throughput for SM with specular reflections. It can be seen that SM shows modest improvement even as the specular reflection coefficient $(\alpha)$ increases. It can be seen that the A. detectors setup shows much better (almost 100\%) performance improvement compared to the $\mathrm{V}$. detectors for the same reflection coefficient $(\alpha=0.5)$. 
Performance analysis of indoor diffuse VLC MIMO channels using Angular Diversity

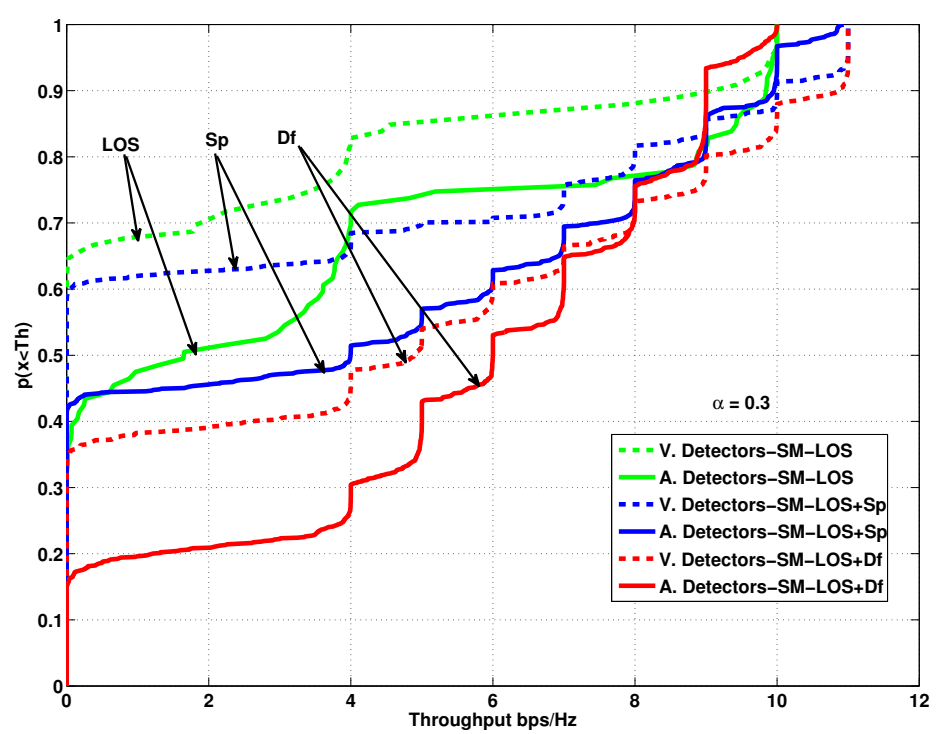

(a)

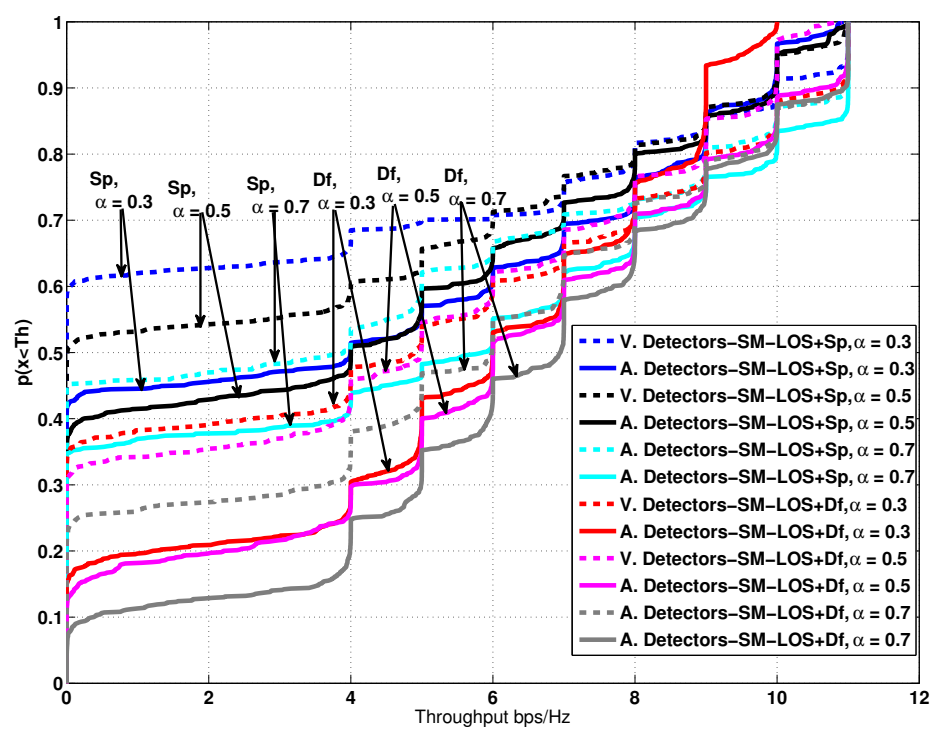

(b)

Figure 5.6: SM CDF comparison between three reflection coefficients in vertically and angular diversity receiver detectors when LOS, LOS $+S p$ and $L O S+D f$ are considered (a) LOS Vs LOS +Sp, $\alpha=0.3$ (b) LOS +Sp Vs LOS + Df, $\alpha=0.3,0.5$ and 0.7 . 
Figure 5.6. shows CDF results for SM where it is shown that both A. Detector and V. detectors have poor performance due to rank deficient conditions in many locations of the room. This problem is addressed by the ASM approach proposed in this chapter.

In Table 5.3 the average throughput for ASM improves as the specular reflection coefficient $\alpha$ increases for both two receiver setups. It is shown that the A. detectors has $17 \%$ throughput improvement compared to V. detectors for the same reflection coefficient $(\alpha=0.5)$. Figure 5.7. shows the CDF results for ASM with three reflection coefficients where it is shown that the A. Detector achieves better coverage and hence throughput improvement. The figure shows that both setups achieve non zero throughput in $100 \%$ of the room locations for this propagation environment. Comparing the results of LOS and the effect of specular reflection at $\alpha=0.5$ it is shown that both setups have $100 \%$ coverage but there is $22 \%$ throughput improvement for A. detectors over V. detectors. Looking at SMP, it shows a significant throughput improvement compared to RC, SM and ASM. This is particularly true because the channel rank for angular diversity detectors is increased to a higher likelihood of LOS propagation between TX and RX compared to vertically oriented detectors and hence a gain in throughput is observed. Taking reflection coefficient $\alpha=0.5$ it is shown that SMP performs $100 \%$ and $175 \%$ better than RC for V. Detectors and A. Detectors respectively and when compared to ASM, SMP performs $59 \%$ and $89 \%$ better for V. Detectors and A. Detectors respectively. It is also noticed that increasing $\alpha$ causes a significant improvement in throughput. Figure 5.8. also shows that when SMP is used there is a significant difference in throughput between the two setups but that coverage of $100 \%$ is achieved for both cases. In the case of specular reflections, the A. detectors shows a $40 \%$ improvement compared to the V. Detector.

\subsubsection{Results for LOS with diffuse reflection channel}

Table 5.4 shows simulation results when diffuse reflections are considered. The diffuse reflection case shows a significant effect on system performance. Considering the $\alpha=0.5 \mathrm{RC}$ results for the diffuse reflection model shows a $47-52 \%$ improvement compared to those for specular reflection (Table 5.3) in both A. detectors and V. detectors. Figure $5.3(\mathrm{~g})$ and $(\mathrm{h})$ show that when diffuse reflections are considered in both $\mathrm{V}$. detectors and $\mathrm{A}$. detectors, the channel matrix rank is always $4(100 \%)$ for all the reflection coefficients $(\alpha=0.1$ through 0.9$)$. 


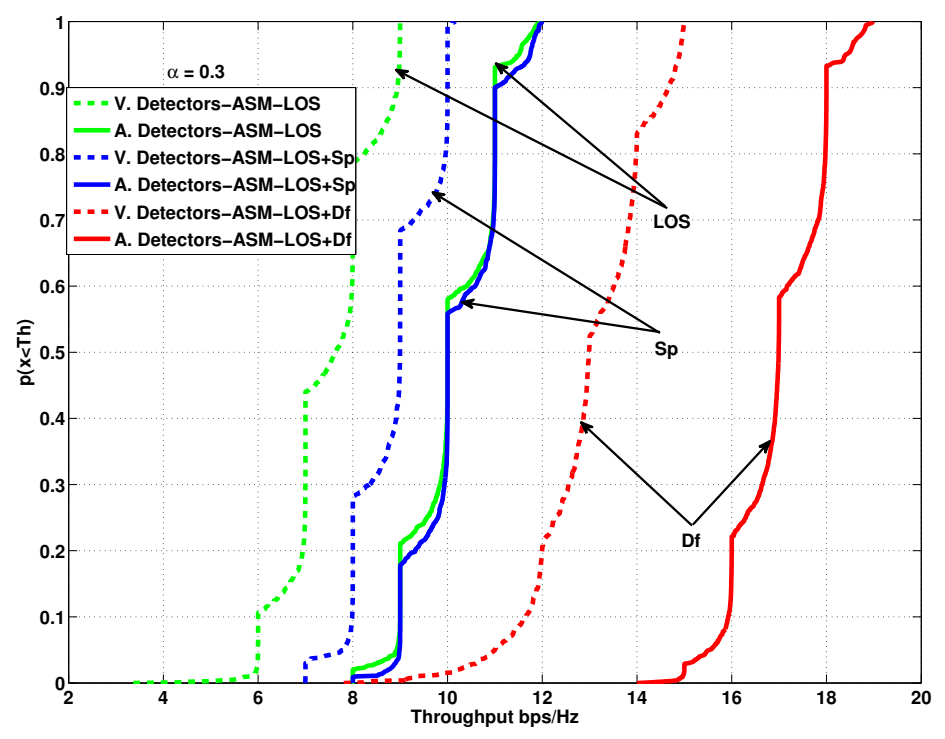

(a)

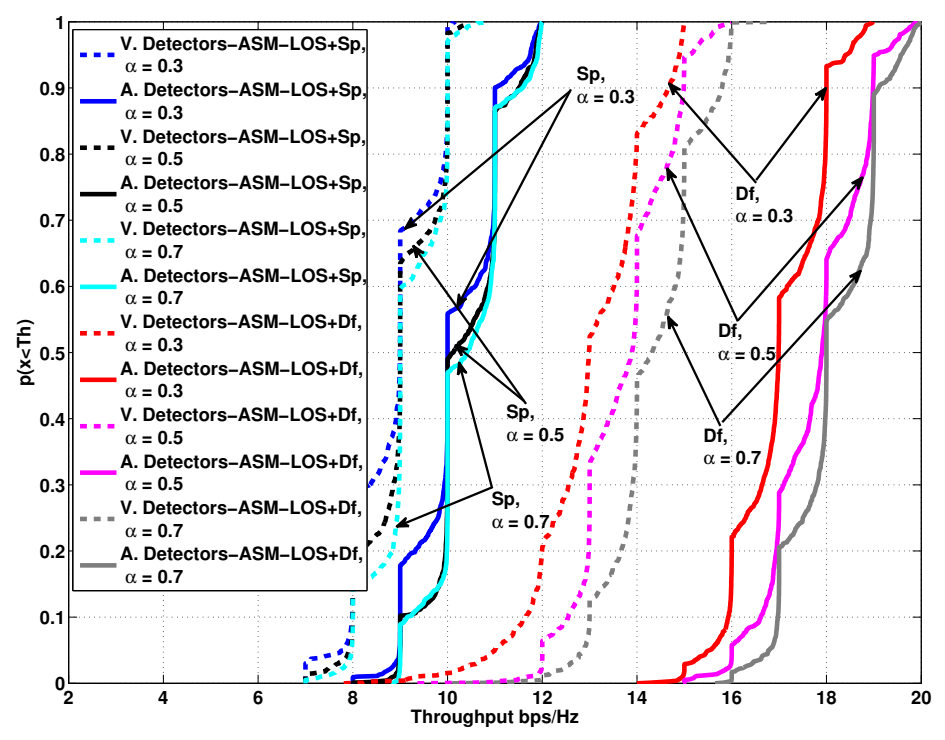

(b)

Figure 5.7: ASM CDF comparison between three reflection coefficients in vertically and angular diversity receiver detectors when LOS, LOS $+S p$ and $L O S+D f$ are considered (a) LOS Vs LOS + Sp, $\alpha=0.3$ (b) LOS + Sp Vs LOS + Df, $\alpha=0.3,0.5$ and 0.7 . 
This is because the diffuse reflection scattered optical channel makes it possible to obtain full MIMO performance gains. Looking at the ASM performance in the model including diffuse reflections, It is also shown a substantial improvement in throughput.

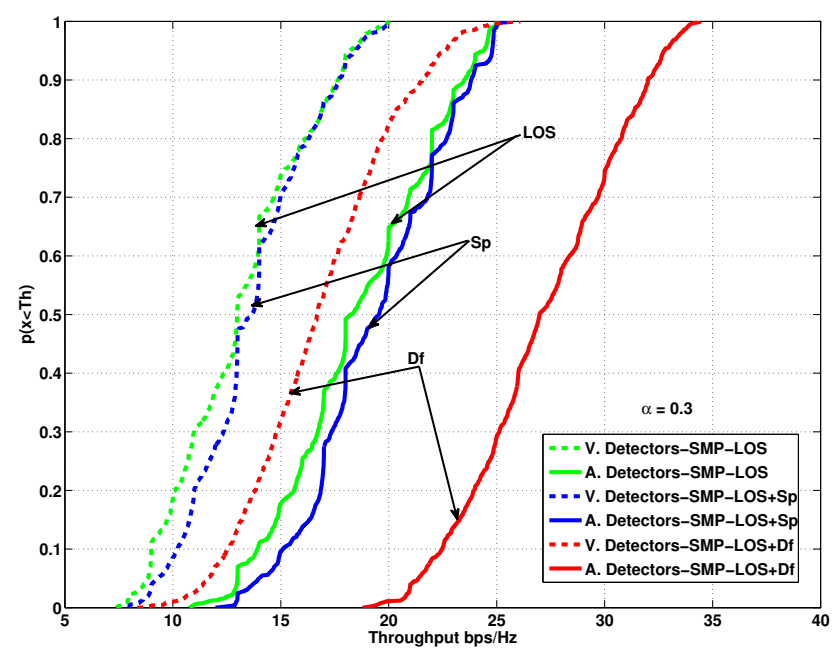

(a)

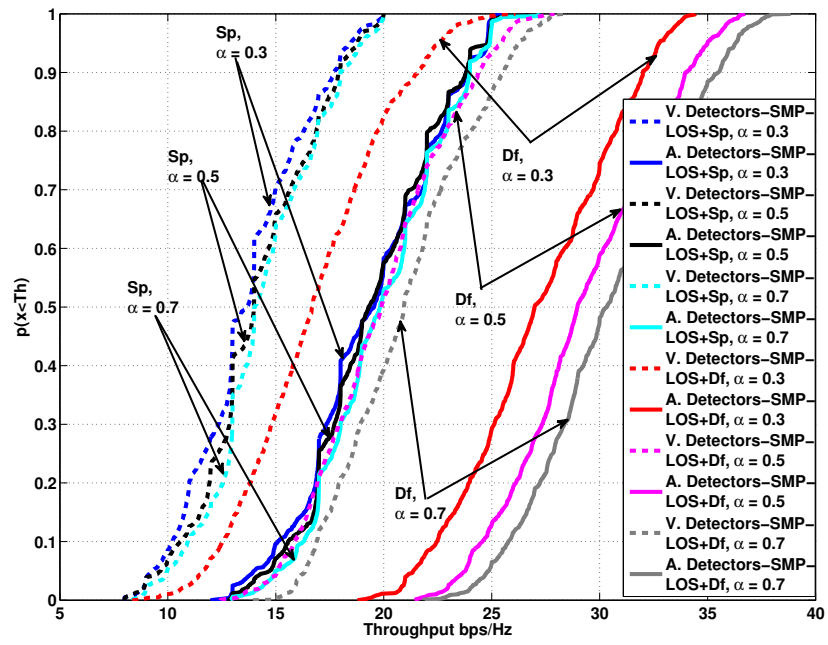

(b)

Figure 5.8: SMP CDF comparison between three reflection coefficients in vertically and angular diversity receiver detectors when LOS, LOS $+S p$ and $L O S+D f$ are considered (a) LOS Vs LOS + Sp, $\alpha=0.3$ (b) LOS + Sp Vs LOS + Df comparison, $\alpha=0.3,0.5$ and 0.7 . 
Table 5.4 shows that ASM ( $\alpha=0.5$ ) has $41-56 \%$ throughput improvement over the specular reflection results in Table 5.3. For SMP, when diffuse reflections are considered it also shows significant improvement. At $\alpha=0.3$ in A. detectors, SMP has an improvement of $20-37 \%$ over the scenario when only specular reflection is included. In terms of the comparison between V. detector and A. detectors in diffuse reflection conditions with $\alpha=0.5$, ASM with A. detectors shows $28 \%$ throughput improvement over ASM with V. detectors while SMP with A. detectors shows $48 \%$ throughput improvement over SMP with V. detectors. Again it is shown that, SMP performs $89 \%$ and $170 \%$ better than RC for V. Detectors and A. Detectors respectively. Generally the diffuse reflection model has shown a big impact on indoor OWC performance due to the of scattered optical power improving the MIMO channel rank statistics.

\subsection{Conclusion}

A mobile angular diversity optical receiver detector model with both specular and diffuse reflections is presented in an indoor visible light communications system. Two different reflection models are applied and a range of reflection coefficients is used to model different types of reflecting surfaces. Different MIMO transmission techniques with adaptive modulation and Per Antenna Rate Control are applied to evaluate the throughput across different room locations. Results for vertical detectors and angular diversity detectors setups in different scenarios are compared. Our initial results show that using angular diversity detectors increase the likelihood of LOS among transmitters and receivers. It is also shown that diffuse reflections have a significant positive impact in system throughput compared to the specular case and both are better than for LOS propagation.

It is shown that for same setup SMP has better performance compared to other candidates (RC, and ASM). Percentage wise, for specular reflections SMP performs $100 \%$ and $175 \%$ better than $\mathrm{RC}$ for V. detectors and A. detectors respectively while in diffuse reflection SMP performs $89 \%$ and $170 \%$ better than RC for V. detectors and A. detectors respectively. Compared to ASM, in specular reflections SMP performs $60 \%$ and $90 \%$ better for V. detectors and A. detectors respectively while in in specular reflections SMP performs $43 \%$ and $66 \%$ better for V. detectors and A. detectors respectively. It was also seen that, using ASM where switching between different TX/RX setups is performed, transmission techniques and modulation, throughput improvements could be achieved compared to always using SM with 4 transmitters.

Looking at both $L O S, L O S+S p$, and $L O S+D f$ results it is shown that ASM performs 
much better than SM due to its robustness to rank deficient channels. In LOS, ASM performs $317 \%$ better than SM with V. detectors and $245 \%$ better than SM with A. detectors. In the specular reflection case when $\alpha=0.5$ is considered, ASM performs $300 \%$ better than SM with V. detectors and $145 \%$ better than SM in A. detectors. Also in the diffuse refection case when considering the same reflection coefficient, ASM performs $168 \%$ better than SM in V. detector and $175 \%$ better than SM in A. detectors. Generally our simulations suggest that, for mobile optical receivers angular diversity detectors can perform better than vertical oriented receivers. When specular or diffuse reflections are included the system performance improves significantly. The positive impact of reflected optical paths on conditions for the scenarios discussed in this chapter is shown. This is because, in the diffuse channels model, receivers can exploit both LOS paths and reflected paths. 


\section{Chapter 6 Conclusion}

The chapter gives a summary and conclusion of the research work presented in this thesis. The major findings are emphasized and the chapters' conclusions are summarised. This chapter also outlines the limitations of this research work and proposes the future work.

\subsection{Summary and major findings}

The rapid growth of data demands which occur as a result of constant increase of wireless communication causes saturation in RF spectrum availability. The consequence of this is the limitation of data transmission rates. This limitation opens doors for researchers to find other alternative ways or complimentary technology to the available RF systems. One of the solutions is to find alternative spectrum resource for wireless communications. The range of spectrum from IR to UV including visible light which gives the total bandwidth resources of about $670 \mathrm{THz}$ (this is a factor 10,000 larger than RF), is thought to offer almost limitless bandwidth which may be useful in wireless communications.

The background for this research was introduced in Chapter 2. A brief history of wireless communication systems was presented. The evolution of radio and mobile communication systems was discussed. The discussion started from the earliest semaphore system developed by Claude Chappe where the position of two arms connected by a cross bar was used to encode information symbol. The discussion was extended to the growing global integration in terms of the 3rd generation partnership project (3GPP) and long-term evolution advanced (LTE-A) collaboration projects. In the discussion, the demand for higher data rates was shown as a driving force for utilization of an alternative spectrum resource. Optical wireless communication (OWC) was introduced, considering geometry, channel link, channel properties, channel models and eye safety consideration. The application of OWC transmission techniques i.e. SC, optical OFDM (O-OFDM) and MIMO were studied.

Analysis and application of SC and O-OFDM in intensity modulation and direct detection (IM/DD) are presented in Chapter 3. Four cell (4-cell) narrow beam systems with applica- 
tions of O-OFDM and SC in different scenarios was developed. The performance of the 4-Cell narrow beam system and a single wide beam were also compared. Our findings showed that the four narrow cell beam (4-cell) can outperform one wide beam LED in terms of coverage area and hence average throughput by the factor of 2.3 (ACO-OFDM), 3 (DCO-OFDM) and 2.8 (PAM) times the single beam system capacity. Also it was shown that sharing the four cells among the available of users results in system channel capacity increase with the number of users up to 4 times the single user single cell capacity. It was also noted that the DCO optimized scheme outperformed the DCO-13dB method at the modulation levels lower than 64 QAM in terms of BER and hence throughput. It was also seen that the optical receiver orientation has a big impact on the system throughput. The throughput improvement achieved after receiver orientation was higher compared to that of the fixed receiver. For the four cell case, ACO saturates in capacity at lower throughput values compared to DCO and PAM.

ASM and angular diversity detectors in an indoor VLC MIMO system was introduced in Chapter 4. The angular receiver setup, MIMO techniques were used and the system throughput evaluation were discussed in this chapter. The throughput results were compared for different receiver detector orientation and transmitter separations. Different MIMO transmission techniques with adaptive modulation and per antenna rate Control were applied in order to evaluate the throughput across different room locations. Results for vertical detectors and angular diversity detectors setups in different scenarios were compared. It was shown that using angular diversity detectors increase the likelihood of LOS conditions among transmitters and receivers. It was also shown that using the ASM technique where different TX/RX setups, transmission techniques and modulation are inter-changed, throughput improvements are achieved compared to always using SM with 4 transmitters. ASM offers throughput improvement of about 4 times that of SM in vertical detectors and about 3 times in angular diversity detectors. For a moving angular diversity receiver there was an improvement in throughput when SMP was used with PARC. Generally the simulations suggested that, for mobile optical receivers, angular diversity detectors can perform better than vertical oriented receivers and ASM improves the SM performance in mobile receivers. Angular diversity detectors offer a better throughput improvement than the state of the art vertical detectors, for example in ASM angular diversity receiver gives throughput of about 1.6 times that of vertical detectors. Also in SMP the angular detectors offer throughput about 1.4 times that of vertical detectors. SMP gives the best performance compared to RC, SM and ASM, for example SMP gives throughput of 2.2 times that of RC in vertical detectors and 3 times in angular diversity receivers. Again SMP gives throughput of 7 
times that of SM in vertical detectors and 6 times in angular diversity receivers. Also SMP provides throughput of 1.7 times that of ASM in vertical detectors and 2 times in angular diversity receivers.

Combination of both LOS and reflections in the channel model was proposed in Chapter 5. Specular and diffuse reflections were modelled in different scenarios using the Lambert-Phong method. Two reflection model techniques using a range of reflection coefficients (modelling of different types of reflecting surfaces) were considered. The total channel gains for either LOS and specular or LOS and diffuse channels can be combined at the receiver detectors. Different MIMO transmission techniques with adaptive modulation and per antenna rate control were used to evaluate the throughput across different room locations. Results for vertical detectors and angular diversity detectors setups in different scenarios were compared. Results showed that using angular diversity detectors increase the likelihood of LOS among transmitters and receivers. It was also shown that diffuse reflections have a significant positive impact in system throughput compared to the specular case and both are better than the LOS. It was also observed that using ASM where different TX/RX setups, transmission and modulation techniques were selected according to channel condition, throughput improvements compared to always using SM with 4 transmitters could be achieved. Further results showed that ASM performs far better than SM due to its capability of combating the rank deficiency problem. In general the simulations suggested that for mobile optical receivers, angular diversity detectors can perform better than vertical oriented receivers. When reflections are included the system performance improves significantly. The positive impact of reflected optical paths on optical propagation for the scenarios discussed in this chapter was shown. In the diffuse channels model, receivers can exploit both LOS paths and reflected paths hence capacity improvement. For example when the reflection coefficient $(\alpha)$ of 0.9 is considered and when the antenna separation is set to $0.6 \mathrm{~m}$, $\mathrm{RC}$ diffuse model shows throughput improvement of about 1.8 times that of LOS for vertical detectors and 1.8 times for angular diversity receivers. SM diffuse model shows throughput improvement of about 3.8 times that of LOS for vertical detectors and 2.6 times for angular diversity receivers. ASM diffuse model shows throughput improvement of about 2 times that of LOS for vertical detectors and 2 times for angular diversity receivers. SMP diffuse model shows throughput improvement of about 1.6 times that of LOS for vertical detectors and 1.7 times for angular diversity receiver. 
The SMP, SM and the newly introduced MIMO transmission and reception techniques in this work (A. Detectors and ASM) are one of effective ways of increasing the spectral efficiency of optical networks. Optical networks can benefit from the proposed A. detectors and ASM.

A detectors has shown a competitive performance in terms of system gain and throughput compared to the state of the art V. detectors. Also the ASM has substantially improved in combating rank deficiency issues compared to the state of the art SM. However the computational complicity of feedback delays and modulation adaptation employed in this work may jeopardise the overall system performance interms of processing time, therefore there is a need of investigation to get overall performance about feedbacks, modulation adaptation schemes and receiver algorithms.

\subsection{Limitations and future work}

- The room dimension used for simulation in this thesis is $4 \mathrm{~m} \times 4 \mathrm{~m}$. When the dimensions are increased there was a significant loss of signal strength. The room dimension may be increased at the expense of transmitter power adjustment, TX/RX-FOV considerations and different TX /RX configurations. These issues require detailled consideration and simulation.

- In a MIMO configuration, the number of transmitters and receivers used were $4 \times 4$ : increasing the number of transmitters has a big impact in computation time. For example when using ML in SM there are $M N_{t}$ possible combinations of transmitter signal while in SMP there are $M^{N_{t}}$ combinations. This combinations consume considerable amount of simulation computation time especially when the higher $M$-level modulation is used and the number of antennas is increased. Methods to reduce detection complexity for SM should be studied.

- The angular four detectors techniques proposed in chapter 4 showed a substantial improvement in MIMO system performance in terms of spectral efficiency. In the future it is suggested to consider additional detectors at the middle of surrounding detectors for the aim of increasing the probability to the receiver.

- The simulation results on this thesis involved application of OFDM and different MIMO transmission methods like RC, SM, ASM and SMP with V. detectors and A. detectors. Also AMC and PARC were employed for improving the system performance in terms of throughput and robustness. Again the QAM and PAM level were used up to 1024-ary 
which is theoretical assumptions as the state of the art systems use 64-ary modulation. However the validation of the results was only based on the analysis of previous work results. Therefore an analytical and experimental framework for verification is need to be established in the future work. 


\section{Appendix A \\ Publications}

\section{A.1 Conference papers}

- P. Fahamuel, J. Thompson and H. Haas, "Study, analysis and application of optical OFDM, single carrier (SC) and MIMO in intensity modulation direct detection (IM/DD)", IET Intelligent Signal Processing Conference 2013, London, pp. 1-6, December. 2013.

- P. Fahamuel, J. Thompson and H. Haas, "Improved Indoor VLC MIMO channel capacity using mobile receiver with angular diversity detectors" IEEE Globe Telecommunications conference (GLOBECOM 2014), Austin, Texas, USA, December. 2014.

\section{A.2 Journal paper}

- P. Fahamuel, J. Thompson and H. Haas, "Performance analysis of indoor diffuse VLC MIMO channels using Angular Diversity Detectors" submitted to IEEE Journal on Light Wave Technology, March. 2015. 


\title{
Study, analysis and application of optical OFDM, single carrier (SC) and MIMO in intensity modulation direct detection (IM/DD)
}

\author{
P Fahamuel *, J Thompson*, H Haas* \\ *The Edinburgh University,Edinburgh EH9 3JL, UK, email: \{p.mmbaga, john.thompson, h.haas\}@ed.ac.uk
}

Keywords: Multi-cell visible light communication (VLC), asymmetrically clipped optical orthogonal frequency division multiplexing (ACO-OFDM), direct current biased OFDM (DCO-OFDM), single carrier (SC).

\begin{abstract}
With the rapid growth of wireless data demands and saturation of radio frequency (RF) capacity, visible light communication (VLC) has become a promising candidate to complement conventional RF communication, especially for indoor short range applications. However the performance of the system depends on the propagation and type of system used. We study four cells optical system where the intensity modulated beam from light sources can be concentrated in specific areas to serve multiple mobile devices with low interference and hence increase system capacity. We then compare the performance of asymmetrically clipped optical orthogonal frequency division multiplexing (ACO-OFDM), direct current biased optical OFDM (DCO-OFDM) and single carrier (SC) modulation schemes in single and multiuser systems.
\end{abstract}

\section{Introduction}

Optical wireless is a communication system which relies on optical radiation to send information through free space, with wavelengths ranging from infrared (IR) to ultraviolent (UV) including the visible light spectrum. The transmitter modulates data and transforms the electrical signal to an optical signal while the receiver demodulates data and converts the incoming optical signal into an electrical current. Light Emitting Diode (LEDs) or Laser Diodes (LDs) can be used as optical transmitters and (PDs) as receivers [1].

Optical communications is proposed due to the fact that it is a viable alternative to Radio Frequency (RF) communications which is becoming very attractive due to large increase of wireless applications. It is attractive because of the low cost and reliability of light sources and receivers. A key advantage is that illumination and communications can take place at the same time, and hence saving power. It is safe to use in places where RF waves are not permitted e.g. hospitals and aircraft [1]. The basic system types fall into diffuse and line of sight (LOS) systems. In a LOS system, high data rates of the order of Gbits/s can be achieved [1,2], but the systems are vulnerable to obstacles/shadowing because of their directionality. In diffuse VLC systems several paths exists from the source to receiver which makes the system robust to obstacles/shadowing. However, the path losses are high and multipath creates inter-symbol interference (ISI) which limits achievable data rates $[3,4]$.

A promising solution to combat the multipath distortion and increase data rate without any bandwidth or power expansion is to use the OFDM (orthogonal frequency division multiplexing) technique. Two OFDM techniques reported in literature are asymmetrically clipped optical orthogonal frequency division multiplexing (ACO-OFDM) and a direct current biased optical OFDM (DCO -OFDM) [5]. In DCO, the bipolar OFDM signal generated by the transmitter is converted to a unipolar signal by adding direct current (DC) bias while in ACO the bipolar OFDM signal is made unipolar by clipping the signal at zero before transmission [5].

In this paper we consider an indoor optical wireless scenario where there are a number of light sources and many users. As the optical links have very limited transmission range caused by noise from ambient light and high path loss, an optical multiuser system is established so that the light beam from the LEDs focus on a specific area to serve different mobile devices with low interference. We then compare performance of optical OFDM and single carrier (SC) techniques for single user and multiuser systems. The rest of the paper is organised as follows. Section 2 presents the basic VLC system description, proposed systems set up scenarios and their relevancy to the study. Systems under consideration, modulations schemes used and simulation scenarios are explained in section 3. Section 4 presents the simulation results. Section 5 concludes the paper and describes the future work.

\section{System description}

In this section we describe the basic optical communication system which comprises one transmitter fixed on the ceiling of the room and is directed downwards perpendicular towards the floor. The receiver is located at table height pointing upward towards the transmitter. Consider a (LOS) optical system propagation path (Fig 1), the channel gain from the transmitter LED to the receiver Photo Diode is given by

$h=\left\{\begin{array}{lc}\frac{A(m+1) \cos ^{m}(\phi) \cos (\theta)}{2 \pi d^{2}}, & 0 \leq \theta \leq \varphi \\ 0 & \theta>\varphi\end{array}\right.$ 


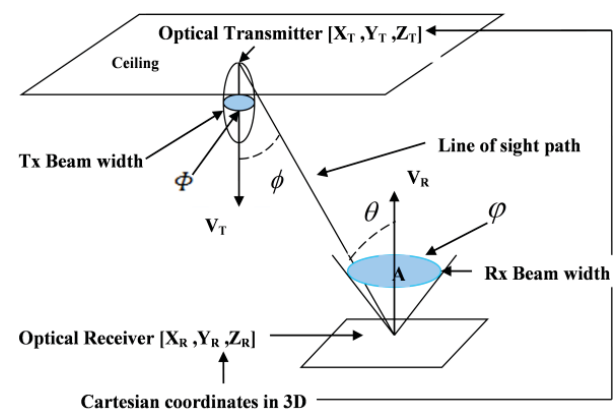

Fig 1: Transmitter and receiver geometry

where $m$ is the order of Lambertian emission, $\phi$ denotes the transmitter viewing angle with respect to the receiving unit, $\theta$ denotes the angle of incidence on the Photo Diode (angle with respect to the transmitter) and $\varphi$ is the receiver field of view (FOV) as shown in Fig 1. $A$ is the collection area of the receiver. $d$ represents the distance between transmitting unit and receiver, and $\Phi$ is the transmitter field of view. $V_{T}$ and $V_{R}$ in Fig 1 are vectors representing the transmitter and receiver normal respectively.

We consider a number of scenarios as follows. The first one is single user-single room light; this was considered to determine the system coverage and channel capacity for one wide beam light with only one user. The second one is single user-multibeam light; this was chosen to determine whether changing the power distribution from one LED cell to 4 LED cells would increase coverage and capacity of the system. Finally, the third model multiuser-multibeam scenario was suggested to determine the advantage of sharing communication among multiple cells in the room.

\subsection{System model}

Consider Fig.1, the received line of sight (LOS) signal vector at any location of the transmitter as in [6] is given by

$$
y_{1}=h_{11} x_{1}+n
$$

Where $x_{1}$ is the transmitted symbol and $y_{1}$ is the received signal. $h_{11}$ is the Lambertian channel coefficient of the transmitter to one user and $n$ represents noise which is assumed to be real valued additive white Gaussian noise (AWGN) with zero mean and double sided spectral density $\delta^{2}$. The power received at the receiver can be calculated as in [6],

$$
P_{r}=P_{t} h_{11}
$$

Where $P_{r}$ and $P_{t}$ are the transmitted and received powers respectively. A photo diode is used to convert the received optical power into an electrical current and the output current is given by

$$
i=P_{r} R
$$

Where $R$ is the photodiode responsivity and the signal to noise ratio (SNR) is given by

$$
S N R=\frac{\left(P_{r} R\right)^{2}}{\delta^{2}}
$$

Where $\delta^{2}$ is a total noise variance calculation as in $[7,8]$, and is given by

$$
\delta^{2}=\delta_{s s}^{2}+\delta_{b g}^{2}+\delta_{d c}^{2}+\delta_{j s}^{2}
$$

$\delta_{s s}^{2}$ is the photon-generated noise or shot-noise in the detector that results from the received signal and is given by

$$
\delta_{s s}^{2}=2 q R P_{r} B
$$

Where, $q$ is the electron charge and $B$ is the electron bandwidth. $\delta_{b g}^{2}$ is the variance caused by background electronic noise power and is given by

$$
\delta_{b g}^{2}=2 q R P_{b g} B
$$

Where $P_{b g}$ is the background radiation power. $\delta_{d c}^{2}$ is a current variance caused by the dark current of the photo diode and is given by

$$
\delta_{d c}^{2}=2 q R I_{d c} B
$$

Where $I_{d c}$ is the dark current in the detector. $\delta_{j s}^{2}$ is the variance in the detector that results from Johnson (thermal) noise and is given by

$$
\delta_{j s}^{2}=\frac{4 K T B F}{R_{l}}
$$

Where $F$ is the noise figure of the system, $T$ is the equivalent temperature, $K$ is the Boltzmann constant and $R_{l}$ is the load resistance. Parameters for modelling Johnson noise are given in Table 1.

\section{Systems under consideration}

All the systems performances of the various scenarios were compared based on optical OFDM and (SC). In an optical wireless system the OFDM signal must be represented as intensity, i.e. the modulating signal must be both real and positive whereas baseband OFDM signal are generally complex and bipolar. A real baseband signal can be generated by constraining the OFDM frequency domain symbol vector to have Hermitian symmetry [5]. The data at the receiver are retrieved using adaptive modulation 
techniques where the computed signal to noise ratio (SNR) at the receiver is fed back periodically to the transmitter and is used to choose the appropriate modulation level.

\subsection{Asymmetrically clipped optical-OFDM}

In ACO-OFDM only odd subcarriers are modulated and even subcarriers as set to zero to ensure that the output consists only real values. For the system with subcarriers 1 to $N$, the data format is

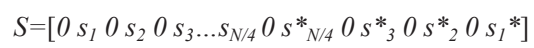

Where $s_{k}$ denotes the data transmitted on the odd subcarrier index $k=1,3,5$, and [.] denotes complex conjugate. The OFDM modulator produces a halfwave symmetric real valued signal. That means the same information in the first half of the samples is repeated in the second half of the OFDM symbol. As a result the negative part can be clipped without any loss of information [5].

The modulation used is quadrature amplitude modulation (4QAM to $1024-\mathrm{QAM}$ ) and the FFT size $(N)$ is 32 . Since only $1 / 4$ of the $N$ subcarriers are useful, the number of bits in one packet (one OFDM symbol) can be calculated by

$$
N_{b}=(N / 4) \log _{2}(M)
$$

Where $M$ is the level of QAM modulation. The packet error probability $(\gamma)$ can be calculated as

$$
\gamma=1-\left(1-P_{e}\right)^{N_{b}}
$$

Where $P_{e}$ is the bit error probability which is the ratio of the number of error bits to the number of transmitted bits. The achieved data rate for the ACO-OFDM system as in $[6,8]$, can be given by

$$
R(A C O)=\frac{(N / 4) \log _{2}(M)}{N}=k / 4
$$

Where $k=\log _{2}(M)$

Therefore the normalised throughput for ACO including packet errors is given by

$$
T_{h}(A C O)=(k / 4)\left(1-P_{p k t}\right) \quad \text { bits } / \mathrm{s} / \mathrm{Hz}
$$

\subsection{Direct current biased optical OFDM}

In DCO-OFDM data are assigned to all odd and even subcarriers except the first and $(N / 2+1)$ subcarriers which must be set to zero to ensure that the output consists only of real values [6]. For the system with subcarriers 1 to $N$, the data format is

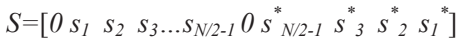

The bipolar OFDM signal is then converted to a unipolar signal by adding a DC bias $\left(\mathrm{B}_{\mathrm{DC}}\right)$ as shown in (17).

$$
x(t)=x_{0}(t)+B_{D C}
$$

Where $x(t)$ is a unipolar signal obtained after adding the DC bias and $x_{0}(t)$ is the bipolar signal. We used two methods of DC biasing, the first one is DCO-13 dB where DC bias voltage level of $13 \mathrm{~dB}$ is used and the second one is called DCO-optimized where three DC biasing voltages levels are used; i.e. $7 \mathrm{~dB}$ for $4,8,16-\mathrm{QAM}, 10 \mathrm{~dB}$ for 32, 34-QAM, and $13 \mathrm{~dB}$ for $128,256,512,1024 \mathrm{QAM}$. The DCO-optimized is proposed because the lower QAM modulation level can achieve low bit error probabilities at low DC bias, which cannot be achieved by high order modulation schemes at the same bias level [3]

The DCO-OFDM biasing DC voltage was calculated based on equation

$$
\left.B_{D C}=k \sqrt{\mathrm{E}\left\{x_{0}^{2}\right.}(t)\right\}
$$

$B_{D C}$ is defined as a bias of $10 \log _{10}\left(k^{2}+1\right) \mathrm{dB}$ [10]. The modulation used is QAM (4-QAM to 1024-QAM) and the FFT size $(N)$ is 32 . Since only $N / 2-1$ of $N$ subcarriers are useful, the number of bits in one packet (one OFDM symbol) can be calculated by

$$
N_{b}=(N / 2-1) \log _{2}(M)
$$

The packet error probability $(\gamma)$ can be calculated as

$$
\gamma=1-\left(1-P_{e}\right)^{N_{b}}
$$

The achieved data rate for DCO -OFDM system, is given by

$$
R(D C O)=\frac{(N / 2-1) \log _{2}(M)}{N}=\frac{N / 2-1}{N} k
$$

Therefore the throughput for DCO is given by

$$
T_{h}(D C O)=\frac{N / 2-1}{N}\left(1-P_{p k t}\right) \quad \text { bits } / \mathrm{s} / \mathrm{Hz}
$$

\subsection{Single Carrier-Pulse Amplitude Modulation (PAM)}

To ensure non-negativity in Pulse Amplitude Modulated symbols, all constellation points must be greater than or equal to zero. For fair comparison the packet length for SC was also set to the length of OFDM symbol for ACO and DCO i.e $N$ symbols.

The modulation used was pulse amplitude modulation (2PAM to 1024-PAM) and the packet size $(N)$ was 32 . For one packet the number of bits can be calculated by

$$
N_{b}=N \log _{2}(M)
$$

The Packet error probability $(\gamma)$ can be calculated as 


$$
\gamma=1-\left(1-P_{e}\right)^{N_{b}}
$$

The achieved data rate for Single carrier (SC) system, is given by

$$
R(S C)=\frac{N \log _{2}(M)}{N}=k
$$

Therefore the throughput for SC is given by

$$
T_{h}(S C)=(k)\left(1-P_{p k t}\right) \quad \text { bits } / \mathrm{s} / \mathrm{Hz}
$$

\subsection{Simulation scenarios}

The simulation scenarios are based on a single LED cell and four LED cells where single and multiple users are considered. These are shown in Fig 2 below

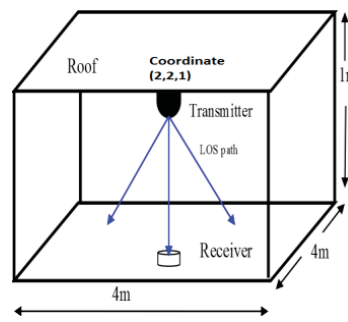

(a)

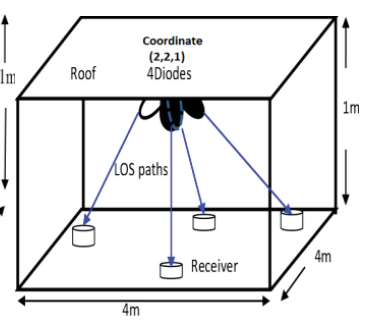

(b)
Fig 2: (a) Single beam light (b) Multibeam lights

\subsubsection{Single user single room light}

Consider Fig 2(a): this scenario aims at finding the data throughput distribution in the different room locations when the LED is pointing straight downwards. In this case the field of view (FOV) of the transmitter and receiver are 180 degrees. The system model for this scenario is based on equation (2) and its signal to noise ratio (SNR) at the receiver is computed using (5).

\subsubsection{Single user multibeam light}

Consider Fig 2(b) with only one user. In this scenario user locations are uniformly distributed in the room. The throughput distribution in the different room locations is calculated when using four LEDs pointing to different angles of the room, at an elevation angle of 45 degrees each and separated from each other by an azimuth angle of 90 degrees. In this case the FOV of the transmitter and receiver are 90 degrees. The received signal vector in any part of the room is given by

$y_{1}=h_{1 j} x_{j}+\sum_{i=1, i \neq j}^{4} h_{1 i} x_{i}+n$

Where the first term of (27) is the LOS signal, while the second terms represents interference from other LEDs. In most of the locations the four interference terms in equation (27) will be equal to zero.

At the receiver the signal to noise and interference ratio (SNIR) is given by

$$
\text { SNIR }=\frac{\left(P_{t} h_{1 j} R\right)^{2}}{\sum_{1=1, i \neq j}^{4}\left(P_{t} h_{1 i} R\right)^{2}+\delta^{2}}
$$

\subsubsection{Multiuser multibeam}

In this scenario we considered 4 cells with multiple users in the room (Fig 2(b)). The aim is to observe how much the system can increase the capacity when users in the room share the cells. The throughput distribution in the different room locations is calculated in each cell and summed up to get the total 4 cell system throughput. In this case the four LEDs and PDs are set as in scenario two. The normalized throughput in each cell is given by

$$
T_{\text {cell }}=1 / U \sum_{i=1}^{U} t_{i}
$$

Therefore the total throughput is given by

$$
T_{\text {full }}=1 / U \sum_{i=1}^{U} \sum_{j=1}^{4} t_{i}
$$

Where, $t_{i}$ is the individual user throughput for user $i$ and $U$ is number of users. $t_{i}$ is computed based on equations (15), (22), or (26) for $\mathrm{ACO}, \mathrm{DCO}$ and $\mathrm{SC}$ respectively. Adaptive modulation is used to obtain the throughput at any user location, i.e. the throughput calculated using different modulation levels for ACO, DCO and SC were stored in lookup table and the maximum throughput determined according to the signal to noise ratio computed at the receiver

\section{Simulation results}

A computer program was written using MATLAB software that implements the scenarios presented in previous sections.

\begin{tabular}{|l|l|}
\hline Parameters & Value \\
\hline Room size $(W \times L \times H)$ & $4 \mathrm{~m} \times 4 \mathrm{~m} \times 1 \mathrm{~m}$ \\
\hline Number of LEDs & 1,4 \\
\hline Vertical distance from ceiling to receiver plane & $1 \mathrm{~m}$ \\
\hline Average transmitted power (per LED) & $27 \mathrm{dBm}$ \\
\hline Transmitter/Receiver field of view $\Phi, \varphi$ & $180,90 \mathrm{deg}$. \\
\hline Photo Diode responsivity $(R)$-eqn $(4)$ & $0.5 \mathrm{~A} / \mathrm{W}$ \\
\hline IFFT length $(N)$ & 32 \\
\hline Electron bandwidth $(B)$-eqn $(7-9)$ & $100 \mathrm{MHz}$ \\
\hline Dark current $\left(\mathrm{I}_{\mathrm{dc}}\right)$ - eqn $(9)$ & $1 \mathrm{nA}$ \\
\hline Back ground radiation power $\left(\delta^{2}\right.$ bg $)$-eqn $(7)$ & $0.1 \mu \mathrm{W}$ \\
\hline Noise figure $(F)$ of the Rx Amplifier-eqn $(10)$ & $5 \mathrm{~dB}$ \\
\hline Transmitter coordinate $(3 \mathrm{D})$ & {$[2,2, \quad 1]$} \\
\hline Photo diode area $(A)$ & $1 \mathrm{~cm}{ }^{2}$ \\
\hline Equivalent temperature $(T)$-eqn $(10)$ & $300 \mathrm{~K}$ \\
\hline Load resistance $\left(R_{l}\right)$-eqn $(10)$ & $10 \mathrm{~K}$ \\
\hline
\end{tabular}


Table 1: Parameters used for simulations.

\subsection{Single user single room light simulation}

Scenario one is simulated using the following parameters: the FOV of transmitter and receiver is 180 degrees. The LED is pointing downward 90 degrees to the horizontal, as shown in Fig 2(a). The system performance is compared based on ACO-OFDM, DCO-OFDM and SC-PAM. 10,000 uniformly distributed locations in the room are used during this simulation. Results are obtained as shown in Fig 3.

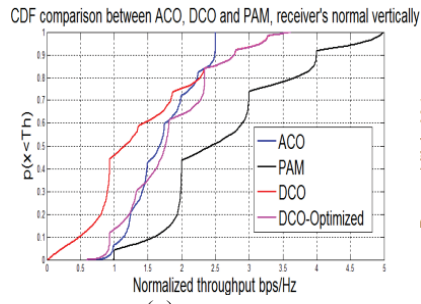

(a)

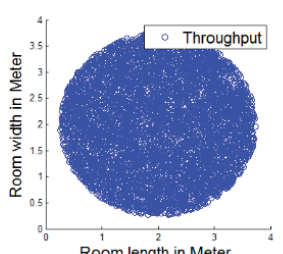

(b)
Fig 3: (a) CDF plot Vs Normalized throughput

(b) Throughput coverage area

Fig 3(a) shows the results of the CDF comparison between ACO, DCO, DCO-Optimized and PAM. The transmitter was pointing downward perpendicular to $\mathrm{x}$, $\mathrm{y}$ plane while the receiver was moving uniformly in the room with its normal pointing upward perpendicular to $\mathrm{x}$, $\mathrm{y}$ plane. Results for this scenario indicate that, PAM promises a good performance compared to other schemes. At the lower modulation level (4QAM to 64-QAM), ACO performs better in spectral efficiency than DCO with a fixed offset level and provides about the same throughput as optimized DCO. Also optimized DCO shows an improvement in performance compared to DCO with a fixed offset level.

Fig 3(b) is a scatterplot of the room throughput distribution for scenario one, i.e. one light beam with one receiver. We can observe that for the room dimension used, the throughput is concentrated at the centre and decreases uniformly towards the walls of the room. The white space in Fig 3(b) denotes the throughput lower than $10^{-4} \mathrm{bits} / \mathrm{s} / \mathrm{Hz}$ which is assumed to be effectively zero throughput. With no considerations of reflections we can also observe regions with no coverage at the corners of the room which indicate the effect of distance on channel gain. This drawback is addressed by the 4 cell system

\subsection{Single user multibeam light simulation}

In this scenario we simulate 4 cells and one receiver with either one, or four detector(s). The scenario provides better coverage by distributing the same power to the lower FOV LEDs. The setup and simulation parameters follow section 3.4.2 and the Rx was moved as in section 4.1. For the case of the receiver the FOV was 180 degrees when only one detector was used and 90 degrees when four detectors were used. For more than one detector, the SNR obtained from each detector was compared and the highest SNR was chosen.

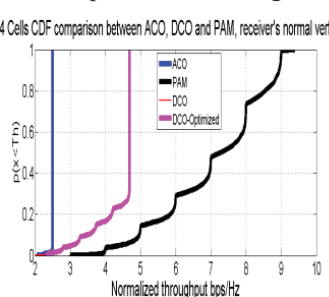

(a)
4 Cells $C D F$ comparison between $A C O, D C O$ and PAM with

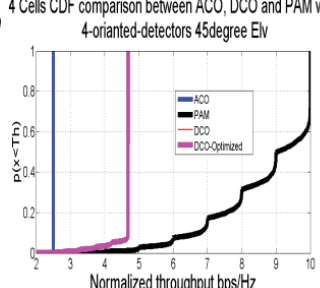

(b)

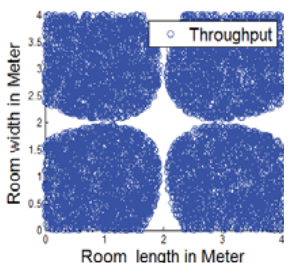

(c)

Fig 4: 4 Cells CDF plot Vs Normalized throughput (a) one detector receiver (b) four detectors receiver (c) Throughput coverage area.

Fig 4 (a) shows the results of the CDF comparison between ACO, DCO, DCO-Optimized and PAM. Results for this scenario indicate that PAM provides a good performance compared to other schemes, also we can see that at higher modulation levels (from 64-QAM above) the DCO-13 $\mathrm{dB}$ and DCO optimized yield the same results. It also shows an improvement in throughput average when compared to one beam light. Looking at Fig. 3(a) we can see that, the average throughput for ACO, DCO and PAM are $1.1 \mathrm{bps}, 1.57 \mathrm{bps}$ and 2.5 bps respectively while for 4 cell systems Fig. 4(a) the average throughput results are $2.5 \mathrm{bps}, 4.7 \mathrm{bps}$ and $7 \mathrm{bps}$ respectively.

Fig. 4(b) shows the results of increasing the number of detectors at the receiver. The aim is to increase the chance of signal detection when either of the detectors points away from any of the 4 LEDs. In this setup the receiver has four detectors inclined in an elevation angle of 45 degrees and separated from each other by 90 degrees. The receiver was allowed to orient randomly over a range of azimuth angles of 90 degrees for each detector which is equivalent to 360 degrees for the whole receiver. When more detectors were added in the receiver the results show that the throughput increases from 7 bps Fig. 4(a) to 9 bps Fig. 4(b) for PAM but remains the same for DCO and ACO because the schemes have reached their maximum possible theoretical throughput results and hit saturation at lower CDF value. However we can see that in DCO the percentage of users that achieve maximum throughput has increased from $70 \%$ Fig4. (a) to $90 \%$ Fig. 4(b).

Fig 4(c) depicts the four beams coverage; here we can see the coverage of each inclined LED and the overall coverage. It shows that, distributing power to 4 LEDs and inclines them at a 45 degree elevation angle causes beams of light to 
concentrate in a specific area hence reduces interference in the multiuser scenario, and also increases room coverage.

\subsection{Multiuser multibeam simulation}

Here the setup is similar to scenario two with multiple users. The simulation considered the throughput calculations for separate cells and summed them up to get full capacity of the system, which is compared with a single user system. The aim is to distribute transmitter power, increase coverage and reduce interference. It this case we assumed only one detector receiver.

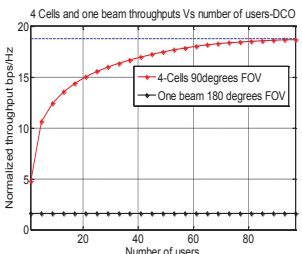

(a)

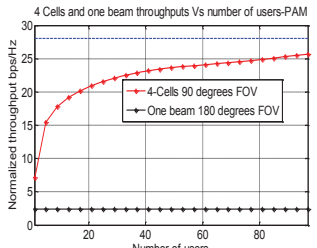

(b)
Fig 5: Normalized throughput Vs Number of users (a) DCOOFDM (b) SC-PAM

Fig. 5(a) shows the results of simulating the DCO- multiuser scenario where the LEDs were set as in scenario two and all receivers with one detector were pointing upward perpendicular to $\mathrm{x}, \mathrm{y}$ plane. Throughput calculations are performed based on Time Division Multiple Acces (TDMA), i.e. using (30). In this scenario we can see the impact of sharing 4 cells amongst multiple users, when we had one by one setup (scenario 3.4.1), the average throughput was 1.75 bps. In scenario 3.4 .2 we distributed power by using 4 cells and the average throughput increased up to $4.7 \mathrm{bps}$. When we shared cells by number of users we can again see that the system channel capacity increases with number of users to four times the single user capacity. This is the maximum single user capacity multiplied by the number of cells in the system. Fig. 5(b) shows the results of simulating PAMmultiuser scenario where the LEDs were set like in scenario two and all receivers with one detector pointing upward perpendicular to $\mathrm{x}, \mathrm{y}$ plane. Throughput calculations was performed as in Fig. 5(a). In this scenario we can also see the impact of sharing 4 cells amongst multiple users, when we had one by one setup (3.4.1), the average throughput was 2.67 bps. In scenario 3.4.2 we distributed power by using 4 cells and the average throughput increased up to $7 \mathrm{bps}$. When we shared cells by number of users we can see that the system channel capacity increases with number of users to four times the single user capacity and it can be seen that for PAM more than 100 users must be accommodated for the system to reach throughput saturation.

\section{Conclusion and future work}

We have presented models and simulated different channel scenarios in indoor visible light communication scenarios. We compared the performance of optical OFDM and single carrier in both single user and multiuser scenarios. Our preliminary findings show that the four narrow cell beams can outperform one wide beam LED in terms of coverage area and hence average throughput. It was also seen that the optical receiver orientation has a big impact on the system throughput Fig. 4(b) and for the four cell case, ACO saturates in capacity at lower throughput values compared to DCO and PAM. In the future we will study the impact of diffuse channels on the results and consider implementation of full Mult-Input Multi-Output (MIMO) techniques for the previous simulated scenarios.

\section{References}

[1] R. Mesleh, H. Haas "Indoor Optical Wireless Communications; Potential and state- of- the art" IEEE Communication magazine, Vol. 49, pp. 56$62,(2011)$

[2] Y. Tanaka et al. "Indoor Visible Light Data Transmission system utilizing White LED lights" IEICE Transaction on communications, Vol 8. pp. 2440-2454, (2003).

[3] J. Armstrong, B. Schmidt "Comparison of asymmetrically clipped optical OFDM and DCbiased Optical OFDM in AWGN" IEEE Communication letters, Vol. 12, pp. 343-345, (2008).

[4] C. Pei et al. "Design of Indoor Optical Wireless Collaborative Cellular system " IEEE Wireless Communications and Signal Processing, Nanjin, Vol. 1, pp.1-6, (2011).

[5] J. Armstrong "OFDM for optical Communications" Journal of light wave technology, Vol. 27, pp. 189-204, (2009).

[6] R. Mesleh, et al "On the Performance of Different OFDM Based Optical Wireless Communication Systems" Journal of optical communication network, Vol. 3, pp. 620-628, (2011).

[7] D. O'Brien et al, “ Improvement of Data rate by using Equalization in an indoor Visible Light Communication system" IEEE conference in Circuits and Systems for Communications, Shanghai, Vol. 4, pp. 678-682, (2008).

[8] J. Tang, Y. Chang "Design of an omnidirectional multibeam Transmitter for High-speed Indoor Wireless Communications" EURASP Journal on Wireless Communications and networking, Vol. 2010 ,pp. 65-75, (2010).

[9] R. Mesleh et al. "An overview of Indoor OFDM/DMT optical Wireless Communication systems "IEEE OWC-11CSNDSP 2010, Newcastle, Vol. 7, pp. 566-570, (2010).

[10] J. Armstrong et al, "Performance of Asymmetrically clipped Optical OFDM in AWGN for an Intensity Modulation Direct detection system" IEEE Globecom proceedings, San Francisco, Vol. 49, pp. 1-5, (2006). 


\title{
Improved Indoor VLC MIMO Channel Capacity Using Mobile Receiver with Angular Diversity Detectors
}

\author{
P. Fahamuel, J. Thompson and H. Haas \\ Institute for Digital Communications, Joint Research Institute for Signal and Image Processing \\ University of Edinburgh, EH9 3JL, Edinburgh UK \\ Email:\{p.mmbaga, john.thompson, h.haas\}@ed.ac.uk
}

\begin{abstract}
A mobile receiver with angular diversity detectors in Visible Light Communication (VLC) Multiple Input Multiple Output (MIMO) channels is considered. The objective is to improve the rank of the channel matrix and hence system throughput. Repetition Coding, Spatial Multiplexing and Spatial Modulation concepts are used to evaluate throughputs across multiple locations in a small room scenario. Since the receiver is mobile, the channel gains are weak in some locations of the room due to the lack of Line of Sight (LOS) paths between transmitters and receivers. Therefore we use Adaptive Modulation and Per Antenna Rate Coding (PARC) to improve spectral efficiency. The throughputs for fixed transmitters and receivers are compared with the oriented/inclined detectors for different cases. The results shows that for the mobile receivers, oriented/inclined detectors with Adaptive modulation and PARC provides capacity improvement when compared with the fixed and vertically oriented receivers.
\end{abstract}

Index terms-Angular diversity detectors; Adaptive Spatia Modulation; Per antenna Rate Control (PARC); Adaptive Modulation and Coding (AMC)

\section{INTRODUCTION}

There has been a rapid increase in demand for wireless data communication and with a saturation of radio frequency (RF) capacity [1] there is a need to find a new spectrum. Thi has stimulated research to find alternative electromagnetic spectrum outside the RF domain. Optical wireless communication, specifically in the visible light spectrum has become a promising candidate to complement conventional RF communications, especially for indoor short to medium range applications. Optical wireless is a communication system which relies on optical radiation to send information through free space, with wavelengths ranging from $10 \mu \mathrm{m}$ (infra-red) to $10 \mathrm{~nm}$ (ultraviolet) including the visible ligh spectrum. The transmitter modulates data and transforms the electrical signal to an optical signal while the receiver demodulates data and converts the optical signal into an electrical current. Light Emitting Diodes (LEDs) or Laser Diodes (LDs) can be used as optical transmitters, and Photo Diodes (PDs) as receivers [1]. The performance of the system depends on propagation condition and the type of the system used. A crucial advantage of optical communications is that illumination and communications can take place at the same time and hence save power. It is safe to use in places where RF is not permitted e.g. in intrinsically safe environments and aircraft [1], also it is attractive because of the reliability and affordability of light sources and receivers.

The parallel usage of multiple transmitters and receivers e.g. OWC Multiple Input Multiple Output (MIMO) can enhance the overall system performance and hence spectral efficiency and reduce a bit error ration (BER) of a communication system [2]. Also MIMO systems can realize a high speed transmission without increasing transmit power and the bandwidth. Multiple Input Multiple Output (MIMO) techniques have been applied to free space optical communications and data transmission to a limited extent. High Data rate MIMO optical wireless communications using white LEDs were proposed in [2], where a fixed receiver with imaging lens was used. It was concluded that the imaging lens and detector array size are large for some configurations and may not to be practical for some applications. Mesleh et al proposed indoor MIMO optical wireless communications using Spatial Modulation (SM) and concluded that reducing both distance between transmitter (TX) and receiver (RX) and transmitter emerging angle (angle between TX axis and the straight line to the receiver) results in lower correlation [3]. Optical MIMO for short range free space data transmission was proposed in [4] and it was shown that MIMO has high potential to improve spectral efficiency. Indoor optical wireless collaborative cellular system was designed in [5] and it was found that, the LOS systems have better performance, but do not provide coverage. In addition to that, analysis on the throughput of the system was considered a future work. Angular diversity of the receiver was studied in [6], [7] for Single Input Single Output (SISO) setup and the results revealed that the Angular Diversity Receiver outperforms the single-PD receiver with regard to Signal to Interference and Noise Ratio (SINR) and Area Spectral Efficiency (ASE). In [8] the performance comparison of MIMO techniques for optical wireless communications in the indoor environment were proposed, the results show that Spatial Multiplexing (SMP) improves the spectrum efficiency where there is low channel correlation. Also it was shown that SM achieves improved spectral efficiencies especially in low signal to noise ratio (SNR) conditions, and it is more robust to channel correlation. Repetition Coding (RC) was found to be insensitive to different transmitterreceiver alignments, but it needs a large signal constellation size to provide high data rates.

In these papers, the movement of the receiver to different locations to see how it affects channel correlation and hence spectral efficiency was not covered. The novel contributions of this paper are:

- Develop angular diversity optical detectors in a MIMO configuration to provide LOS channel con- 
ditions in more locations of the room and compare its performance with that of vertical detectors.

- Devise a novel adaptive form of Spatial Modulation which is robust to rank deficient channels in different locations of the room

- $\quad$ Provide performance statistics for MIMO methods operating over many room locations, using Adaptive Modulation and Coding (AMC) with an optical form of Per Antenna Rate Control.

The rest of the paper will be as follows. Section II presents the system model. Section III explains the MIMO techniques used in this paper. Section IV introduces the models for the system throughput evaluation. Section V introduces the simulation scenarios and discuses the obtained results. Section VI gives conclusions and future work ares.

\section{SYSTEM MODEL}

Visible Light Communications (VLC) MIMO transmission is considered where Intensity Modulation (IM) and Direct Direction (DD) of the optical carrier using incoherent light source are employed. The system consists of $N_{t}$ transmitters and $N_{r}$ photo-detectors at the receiver side. The received signal vector is:

$$
\mathbf{y}=\mathbf{H s}+\mathbf{n}
$$

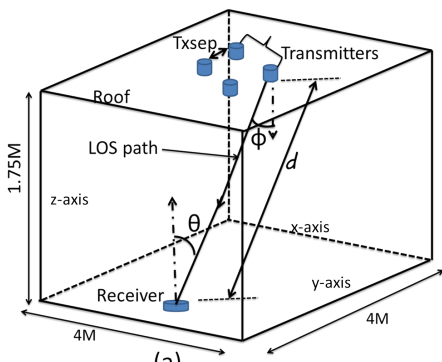

(a)

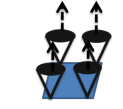

(b)

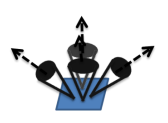

(c)
Fig. 1. Transmitter and receiver geometry used for channel coefficient calculations (a) General setup (b) Receiver with vertical axis detectors (c) Receiver with inclined axis detectors

where $\mathbf{s}$ is the transmitted signal vector which is defined as follows: $\mathbf{s}=\left[\begin{array}{lll}s_{1} & \ldots \ldots s_{N t}\end{array}\right]^{T}$ with [.] ${ }^{T}$ being the transpose operator and $s_{n}$ denotes the signal transmitted by $n^{\text {th }}$ LED. The sum of ambient shot light noise and thermal noise is denoted by an $N_{r} \times 1$ vector $\mathbf{n}$ which is assumed to be real valued additive white Gaussian noise (AWGN) with zero mean and variance:

$$
\delta^{2}=\delta_{\text {shot }}^{2}+\delta_{\text {thermal }}^{2},
$$

where $\delta^{2}$ shot is the shot noise variance and $\delta^{2}$ thermal is the thermal noise variance. Thus the noise power is given by $\delta^{2}=N_{0} B$, where $N_{0}$ is the noise power spectral density and
$B$ is bandwidth. The $N_{r} \times N_{t}$ channel matrix $\mathbf{H}$ for each room coordinate $(x, y, z)$ is given by:

$$
\mathbf{H}(x, y, z)=\left(\begin{array}{ccccc}
h_{11(x, y, z)} & \cdot & \cdot & \cdot & h_{1 N t(x, y, z)} \\
\cdot & \cdot & & & \cdot \\
\cdot & & \cdot & & \cdot \\
\cdot & & & \cdot & \cdot \\
h_{N r 1(x, y, z)} & \cdot & \cdot & \cdot & h_{N r N t(x, y, z)}
\end{array}\right)
$$

where $h_{n_{r} n_{t}}$ represents the channel transfer function of the wireless link between transmitter $n_{t}$ and receiver $n_{r}$ at location $(x, y, z)$. The path difference between multiple transmitter-receiver links is, typically a few $\mathrm{cm}$ as shown in Fig. 1. Therefore, the delay between the different links is negligible. Consequently we consider that the delay spread of the channel is much smaller than the symbol period. In this paper we assume an optical wireless link with LOS characteristics (Fig. 1). As illustrated $\phi$ is the angle of emergence with respect to the transmitter axis, $\theta$ is the angle of incidence with respect to the receiver/detector axis and $d$ is the distance between transmitter and receiver. Consider a LOS optical system propagation path in Fig. 1, the channel gain from the transmitter to the receiver is given by [8]:

$$
h=\left\{\begin{array}{cc}
\frac{A(k+1) \cos ^{k}(\phi) \cos (\theta)}{2 \pi d^{2}}, & 0 \leqslant \theta \leqslant \varphi_{1 / 2} \\
0, & \theta>\varphi_{1 / 2}
\end{array}\right.
$$

$$
\text { where } k=\frac{-\ln (2)}{\ln \left(\cos \left(\Phi_{1 / 2}\right)\right)}
$$

$\Phi_{1 / 2}$ is the transmitter semi-angle (at half power), which is assumed to be $45^{\circ}$. The scalar $\varphi_{1 / 2}$ is the Field of View (FOV) semi angle of the receiver which is also assumed to be $45^{\circ}$. In [8] $\Phi_{1 / 2}$ and $\varphi_{1 / 2}$ were assumed to be both $15^{\circ}$. When narrower half angle is used for this setup (where the receiver is moving), it is difficult to get LOS channel conditions in many locations of the room, that is the reason we use $45^{\circ}$ instead. $A$ is the collection area of the receiver $n_{r}$. We further assume that $h_{n_{r} n_{t}}=0$, when a receiver and transmitter are not in each others FOV.

Fig. 1(c) shows the receiver with inclined detectors. To achieve this setup the detector axis vector $\mathbf{z}$ which is inclined in an elevation angle $45^{\circ}$ is transformed around the $z$ axis (vertical axis) using the transformation matrix such that

$$
\begin{aligned}
& \hat{\mathbf{z}}=\quad R_{\mathbf{z}}(\vartheta) \times \mathbf{z} \\
& \left(\begin{array}{l}
\hat{x} \\
\hat{y} \\
\hat{z}
\end{array}\right)=\left(\begin{array}{ccc}
\cos \vartheta & -\sin \vartheta & 0 \\
\sin \vartheta & \cos \vartheta & 0 \\
0 & 0 & 1
\end{array}\right)\left(\begin{array}{l}
x \\
y \\
z
\end{array}\right)
\end{aligned}
$$

where $\hat{\mathbf{z}}$ is the transformed vector, $\vartheta$ presents the transformation angle around the $z$ axis for an interval of $90^{\circ}$ to form detectors' axis vectors pointing at azimuth angles $0^{\circ}, 90^{\circ} 180^{\circ}$ and $270^{\circ}$ and $R_{\mathbf{z}}$ is the transformation matrix with respect to $z$ axis. Since any variation of detector's axis affects the angle of incidence $\theta$ as (4), each detector elevation angle is varied from $45^{\circ}$ to $90^{\circ}\left(0^{\circ}\right.$ from vertical) to find the elevation angle that will maximize the rank of channel 
matrix $\mathbf{H}$. Therefore the selected angle is used for system performance evaluation.

An indoor $4 \times 4$ MIMO scenario $\left(N_{r}=4\right.$ and $\left.N_{t}=4\right)$ as in [8] is considered. The system is located within a room of size $4 \times 4 \times 3 \mathrm{~m}$ and it is assumed the transmitters are placed at a height of $2.50 \mathrm{~m}$ and oriented downwards perpendicular to the floor of the room. The receiver is allowed to move randomly at a height of $0.75 \mathrm{~m}$ (human waist or table height) and its detectors oriented in a variable elevation angles as in Fig. 1 (c). The inclined detector's orientation is mean to increase the FOV between transmitters and receivers and hence increase system spectral efficiency.

\section{MIMO TECHNIQUES}

Three different MIMO techniques are used; Repetition coding (RC), Spatial Modulation (SM) and Spatial Multiplexing (SMP) as in [8]. In contrast to [2], [3] and [4] where fixed receivers are used, in this paper we consider that mobile receiver is able to move freely around the room. To overcome the resulting SNR variations we employ Adaptive Modulation with Per Antenna Rate Control (PARC) [9] in SMP and Adaptive modulation in SM and RC. In these techniques, the modulation $M$-level is chosen and updated in each transmit time depending on the current channel conditions. We assume that all considered MIMO techniques use Maximum Likelihood (ML) [8] detection at the receiver with perfect knowledge of the channel and ideal time synchronisation. Therefore the decoder selects a constellation vector $\hat{s}$ which minimizes the Euclidean distance between the actual received signal $\mathbf{y}$ and all the possible signal vectors leading to

$$
\hat{s}=\arg \underset{s}{\max } p_{\mathbf{y}}(\mathbf{y} \mid \mathbf{s}, \mathbf{H})=\underset{s}{\arg \min }\|\mathbf{y}-\mathbf{H s}\|_{F}^{2}
$$

where $p_{\mathbf{y}}$ is the probability density function of $\mathbf{y}$ conditioned in $\mathrm{s}$ and $\mathbf{H}$. $\|.\|_{F}$ denotes the Frobenius norm.

\section{A. Repetition coding $(R C)$}

The first technique used is RC which simultaneously emits the same signal from all transmitters. Therefore the condition $s_{1}=s_{2}=\ldots . . s_{N t}$ holds [8]. In RC the intensity coming from the several transmitter constructively adds up at the receiver side. In this paper unipolar $M$-level Pulse Amplitude Modulation ( $M$-PAM) is considered together with RC, where $M$ denotes the signal constellation size. Therefore $M$-PAM achieves a spectral efficiency of $\log _{2}(M)$ bit/s/Hz. PAM is more bandwidth efficient compared to other pulse modulation techniques like Pulse-width Modulation (PWM), On-off Keying (OOK), Pulse -Position Modulation (PPM) etc. [8]. We employ rectangular pulse shapes with $M$-PAM. The intensity level emitted by the Light Emitting Diode (LED) is given by:

$$
I_{m}^{\mathrm{PAM}}=\frac{2 I}{M-1} m, \text { for } m=0,1, \ldots \ldots . .(M-1)
$$

where $I$ is the mean optical power emitted. The Bit Error Rate (BER) for unipolar $M$-PAM can be expressed by

$$
\mathrm{BER}_{\mathrm{PAM}} \geqslant \frac{2(M-1)}{M \log _{2}(M)} Q\left(\frac{1}{M-1} \sqrt{\frac{E_{R X}}{N_{0}}}\right)
$$

where:

$$
Q(a)=\frac{1}{\sqrt{2 \pi}} \int_{a}^{+\infty} \exp \left(\frac{-t^{2}}{2}\right) d t
$$

is the $Q$ function [8] and $E_{R X}$ is the received electrical energy. The BER of $M$-PAM given in (8) can be generalized for the $N_{r} \times N_{t}$ scenario. The BER of RC is given in equation (7) of [8].

\section{B. Spatial Modulation (SM)}

The second used technique is SM, which combines MIMO and digital modulation proposed in [10] and further investigated in [11], [12]. In SM the conventional constellation diagram is extended to an additional dimension namely, the spatial dimension. Specifically, the LED index is used to transmit bits. Each transmitting LED is assigned a unique binary sequence (the spatial symbol). A transmitter is only activated when the random spatial symbol to be transmitted matches the pre-located spatial symbol. Thus only one transmitter is activated for any PAM symbol transmission. Therefore only one element of the signal vector $s$ to be transmitted is non-zero. SM provides an enhanced spectral efficiency of $\log _{2}\left(N_{t}\right)+\log _{2}(M)$ bit/s/Hz. Signals with intensity $I_{m}=0$ can not be used for the signal modulation of SM. Because in this case no transmitter would be active and the spatial information information would be lost [8]. Therefore, the intensities of common PAM given in (7) has to be modified to be suit SM leading to:

$$
I_{m}^{\mathrm{SM}}=\frac{2 I}{M+1} m, \text { for } m=1, \ldots \ldots . .(M)
$$

BER expression for SM is given in (13) of [8].

C. Adaptive Spatial Modulation (ASM)

In this paper we propose adaptive SM which is developed to cope with rank deficient channels. The reason for implementing Adaptive SM is that, when the receiver moves around the room the channel matrix $\mathbf{H}$ is not full rank (4) in many locations. If channel matrix rank is reduced, the receiver can not easily distinguish all transmitting LEDs. In Adaptive SM, the receiver checks the rank of the channel matrix and decides which TX/RX setup to be used. Three modes are used, for example:

- If $\operatorname{rank}(\mathbf{H})=4$, four transmitter Adaptive Modulation $\mathrm{SM}$ are used for transmission and throughput calculations.

- If $\operatorname{rank}(\mathbf{H})=2$ or 3, 2TX Adaptive Modulation SM is used. To identify which two transmitters are to be activated we check the $\mathbf{H}$ matrix using the brute force search method [13].

- If $\operatorname{rank}(\mathbf{H})=1, \mathrm{RC}$ with Adaptive Modulation is used. 


\section{Spatial Multiplexing (SMP)}

The third MIMO technique is SMP. In SMP independent data streams are simultaneously transmitted from all the transmitters. So, SMP provides a maximum spectral efficiency of $N_{t} \log _{2}(M)$ bit/s/Hz. Similar to RC, PAM is used with SMP and equally distributes the optical power across all emitters to ensure that both schemes use the same mean transmission power. The BER for SMP is given in (10) of [8].

\section{System throughPUt EVALUATION}

For the RC techniques the BER Expression described in [8] is used and throughput calculation approach given in [6] is used:

$$
T_{h(\mathrm{SISO})}=R(1-\gamma) \quad \mathrm{bps} / \mathrm{Hz}
$$

where $R$ is the maximum rate of the scheme, $\gamma$ is the packet error probability which is given by [6]:

$$
\gamma=1-\left(1-P_{e}\right)^{N_{b}}
$$

where $P_{e}$ is a bit error probability and $N_{b}$ is the number of bits in one packet.

The BER value computed is minimized such that

$$
\mathrm{BER}_{\text {best }}=\arg \min \left(\mathrm{BER}_{\mathrm{RC}}\right)
$$

This is computed for each $M$-level that will result in BER less than $10^{-2}$ (Fig. 3). Then, using the minimum BER obtained, the throughput for each $M$-level is determined and the highest one is selected.

For SMP adaptive modulation with PARC [9] is used. In PARC, the choice of modulation is optimized separately for each transmitter. The BER expression as in [8] is computed and the highest modulation level is chosen such that

$$
\text { BER }_{\mathrm{TX}_{i m}}<10^{-2}
$$

where $T X_{i}$ denotes the $i^{\text {th }}$ transmitter and $m$ is the highest modulation level selected for $i^{\text {th }}$ transmitter in a given SNR. BER values greater than $10^{-2}$ are ignored since these will not give a substantial throughput gain, as shown in Fig. 3. and therefore the overall throughput is computed using:

$$
T_{h(\mathrm{MIMO})}(x, y, z)=\sum_{i=1}^{N_{T}} T h_{\mathrm{BER}_{\mathrm{TX}_{i}{ }_{M i}}}
$$

Where $T h_{\mathrm{BER}_{\mathrm{TX}{ }_{M} M_{i}}}$ denotes throughput for an individual LED with an appropriate modulation while $N_{T}$ presents the number of transmitting LEDs.

For SM, after the process explained in subsection (c) of (III) has been performed, the BER calculations is carried out using (13) of [8], and the technique for selecting the best BER is the same as (14). Overall throughput is calculated using (15), with the difference that $T X_{i}$ denotes $i^{\text {th }} \mathrm{TX} / \mathrm{RX}$ setup and $M_{i}$ is the highest modulation level selected for $i^{t h}$ TX/RX setup in a given SNR.

\section{Simulation PARAMETERS AND RESUltS}

The simulation parameters are tabulated in Table I. The room size shows the dimension of the room where the simulation is assumed. TX/RX setup presents the number of transmitters and receivers used in MIMO configuration. TX separations shows how far the four transmitting LEDs are separated from each other (here the three separations were simulated). RX separation shows how the receiver detectors are separated (this applies for vertically oriented receiver). TX/RX FOV are the Field of View angles of transmitter and receiver respectively. RX detectors elevation angle is the one that detector's axis is directed. For vertically oriented detectors the angle of elevation is zero. RX detectors azimuth angle separations shows how four detectors are angular separated around $360^{\circ}$ for the angular diversity detectors (A. Detectors). As for the vertically oriented receiver (V. Detectors) the angle is always zero from the vertical.

TABLE I. PARAMETERS USED FOR SIMULATION

\begin{tabular}{|c||c|}
\hline \multicolumn{1}{|c||}{ Parameters } & Values \\
\hline Room size $(W \times L \times H)$ & $4 \mathrm{~m} \times 4 \mathrm{~m} \times 3 \mathrm{~m}$ \\
\hline TX/RX setup & $4 / 4$ \\
\hline TX separations & $0.2 \mathrm{~m}, 0.4 \mathrm{~m}, 0.6 \mathrm{~m}$ \\
\hline RX separations (V. detectors) & $0.1 \mathrm{~m}$ \\
\hline TX/RX FOV & $45^{\circ}, 45^{\circ}$ \\
\hline RX elevation angle (A. Diversity) & $45^{\circ}$ to $90^{\circ}$ towards vertical \\
\hline RX azimuth angle separation (A. Diversity) & $90^{\circ}$ \\
\hline Photodiode area ( $A$ ) & $1 \mathrm{~cm}^{2}$ \\
\hline MIMO transmission techniques & RC, SMP, SM \\
\hline Modulation schemes & 2 PAM-1024PAM \\
\hline
\end{tabular}

\section{A. Vertical detectors setup}

To validate results, the setup developed in [8] was repeated for all proposed MIMO transmission techniques and different TX separations $(0.2 \mathrm{~m}, 0.4 \mathrm{~m}, 0.6 \mathrm{~m})$. RX detectors separation remains $0.1 \mathrm{~m}$ all the of time with a vertically oriented RX as in Fig. 1(b), assuming installation of the detector array in typical laptops. The BER results for a data rate of $\mathrm{R}=4 \mathrm{bps} / \mathrm{Hz}$ are given in Fig. 2. These results match closely with Fig. 3(a) of [8] and verify the validity of the simulator.

The mobile receiver model was then developed and the throughput for 1000 locations was evaluated. In this case of a mobile receiver adaptive modulation is used for all of the transmission techniques, i.e. the rate of transmission is updated periodically depending on channel condition. In addition to adaptive modulation, PARC in SMP is considered. The results of average throughputs for different transmission techniques and different TX separations are compared in Table II and CDF for both techniques are in Fig. 5. Both Table data and CDF plots are explained in subsection (c) of (V).

\section{B. Angular diversity detectors setup}

The concept of orienting detectors in different directions is implemented, in order to improve the likelihood of LOS propagation between the TX and the RX and hence increasing spectral efficiency. The setup of TX and transmission techniques remain as in section $A$ but the receiver setup is 


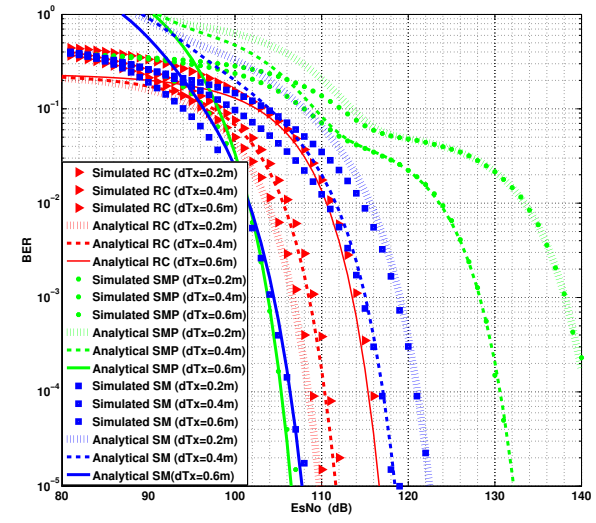

Fig. 2. BER results (analytical and simulation) for MIMO vertical fixed detectors setup with TX separations of $0.2 \mathrm{~m}, 0.4 \mathrm{~m}$ and $0.6 \mathrm{~m}$ employing RC SMP and SM (compare with Fig. 3(a) in [8]). As the channel coefficients of the considered setups are in the range of $10^{-4}$, the electrical path loss is about $-80 \mathrm{~dB}$

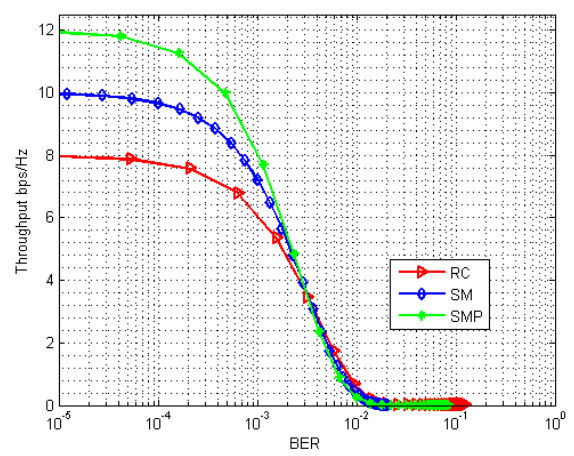

Fig. 3. Throughput Vs BER curves for SMP, SM and RC for angular diversity mobile receiver at $(2.05,1.95,0)$

modified. The coordinates $(x, y, z)$ of the detectors are the same for all of the four detectors but they are oriented at different elevation and azimuth angles. The azimuth separation between neighbouring sensors is $90^{\circ}$ to cover $360^{\circ}$ azimuth, and the elevation angle is varied from $45^{\circ}$ to $90^{\circ}$ as in section II. The best angle is selected and the throughput is calculated for 1000 locations. The elevation angle of $75^{\circ}$ ( $15^{\circ}$ from vertical) for each detector was found to give a substantial capacity improvement and therefore is used for comparison with the vertical receiver.

C. Comparison between Vertical detectors and Angular diversity detectors scenarios

The performance of vertical detectors and angular diversity detectors for a mobile receiver is compared. All of the setups are simulated using all of the MIMO transmission techniques given in section III. Fig. 4(a) shows that, the

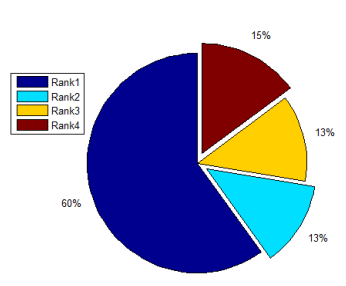

(a)

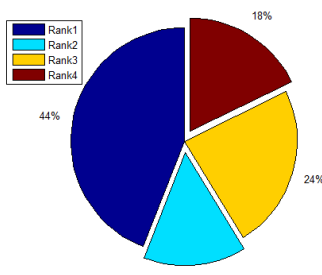

15
Fig. 4. The percentage distributions of MIMO channels matrix rank in 1000 locations of the room (a) Vertical detectors setup (b) Angular diversity detectors setup

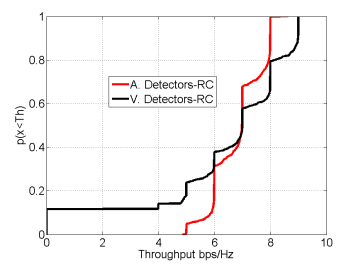

(a)

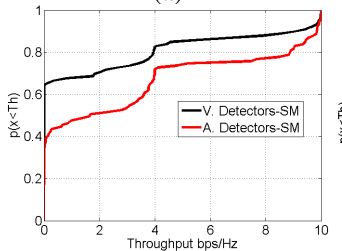

(c)

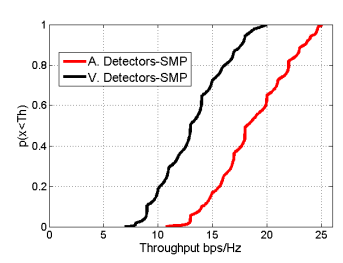

(b)

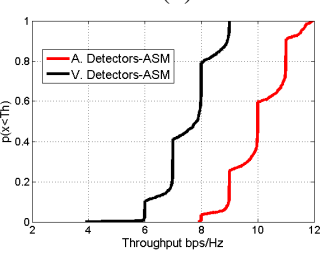

(d)
Fig. 5. CDF comparison between three MIMO techniques in vertically and angular diversity receiver detectors (a) RC (b) SMP (c) SM (d) ASM

TABLE II. Simulation Results

\begin{tabular}{|c||c|c|c|c|c|c|}
\hline \multirow{2}{*}{$\begin{array}{c}\text { Trans- } \\
\text { mission } \\
\text { Method }\end{array}$} & \multicolumn{5}{|c|}{ Average throughput in bps/Hz } \\
\cline { 2 - 7 } & $\mathbf{0 . 2} \mathbf{~ m}$ & $\mathbf{0 . 4} \mathbf{~ m}$ & $\mathbf{0 . 6} \mathbf{~ m}$ & $\mathbf{0 . 2} \mathbf{~ m}$ & $\mathbf{0 . 4} \mathbf{~ m}$ & $\mathbf{0 . 6} \mathbf{~ m}$ \\
\hline RC & 5.2 & 5.6 & 6 & 6.5 & 6.3 & 6.4 \\
\hline SM & 1.1 & 1.3 & 1.8 & 1.7 & 2.2 & 2.9 \\
\hline ASM & 5.2 & 5.8 & 7.6 & 6.1 & 7 & 10.1 \\
\hline SMP & 6 & 8.2 & 13.2 & 13 & 15.4 & 18.7 \\
\hline
\end{tabular}

$\mathrm{V}=$ Vertically oriented, $\mathrm{A}=$ Angular diversity

channel matrix rank for the vertical detector is 1 for a large area of the room $(60 \%)$; Fig. 4(b) the proportion is reduced for the angular diversity detectors $(44 \%)$ indicates higher potential for MIMO receivers. Typical average throughput results for all setups (vertically oriented and angular diversity detectors) and their scenarios (TX separations) are in Table II.

Results are shown for four techniques (RC, SM, ASM and SMP), with both V. Detector (vertically oriented detectors) and A. Detector (angular diversity detectors) configurations for three transmitter spacings.

The $\mathrm{RC}$ results show that the average throughput for all 
setups does not vary significantly because the same data are transmitted for all the transmitting LEDs so the same data rate is achieved whether the rank of channel matrix is 1 or 4 , Fig. 5(a) shows the CDF comparison between V. Detectors and $\mathrm{A}$. Detectors in $\mathrm{RC}$ and the difference between two setups mainly relates to the coverage where the V. Detectors setup serves 10\% fewer locations than the A. Detectors setup.

The SM results shows that, the average throughput is worse because of the channel rank deficiency as explained in subsection (c) of section III. Fig. 5(c) shows that only $60 \%$ and $35 \%$ of the locations is covered for angular oriented detectors and vertical oriented detectors setups respectively. This poor performance is addressed in Adaptive Spatial Modulation.

The average throughput for ASM improves SM performance when the two receiver setups are simulated. It is shown that A. Detectors has $39.5 \%$ throughput improvement compared to V. Detectors for the same TX separation $(0.6 \mathrm{~m})$. This improvement is achieved because of changing transmission techniques and setups according to the channel matrix rank as explained earlier. In Fig. 5(d) the CDF results for ASM shows that the A. Detector achieves better coverage and hence throughput improvement. Fig. 5(d) shows that both setups cover $100 \%$ of the locations and that the A Detectors shows better performance than the V. Detectors. The results of SM and ASM for a similar TX separation $(0.6 \mathrm{~cm})$ show that both setups have average throughput improvements greater than $100 \%$.

SMP shows a significant throughput improvement compared to RC, SM and ASM. This is particularly true when compared with vertically oriented detectors the channel rank for angular diversity detectors is increased to the higher likelihood of LOS propagation between TX and RX a gain in throughput is achieved. Note that increasing the TX separation decreases the channel correlation and gives significant improvement of throughput as transmitter separation is increased. For the same separation $(0.6 \mathrm{~m})$, A. Detectors has a $42 \%$ throughput improvement compared to V. Detectors. Fig. 5 (b) also shows that when SMP is used, there is a significant difference in throughput between the two setups, and for both cases, the coverage is $100 \%$.

\section{CONCLUSION}

A mobile angular diversity optical receiver detector model in an indoor visible light communications is presented. Different MIMO transmission techniques with adaptive modulation and Per Antenna Rate Control are applied in order to evaluate the throughput across different room locations. Results for vertical detectors and angular diversity detectors setups in different scenarios are compared. Initial results show that using angular diversity detectors increase the likelihood of LOS among transmitters and receivers as shown in Table II, Fig. 4 and Fig. 5. Also for a moving angular diversity receiver there is an improvement in throughput when using SMP with PARC (Table II). It is shown that for same setup, SMP has better performance compared to other candidates (RC, SM and ASM) as it is detailed in subsection (c) of (V). Percentage wise, for the same TX separation, SMP performs $120 \%$ and $192 \%$ better than RC for V. Detectors and A. Detectors respectively. Compared to ASM, SMP performs $78.9 \%$ and $85.1 \%$ better for $\mathrm{V}$. Detectors and A. Detectors respectively. It was also seen that, using ASM where different TX/RX setups, transmission techniques and modulation are inter-changed, throughput improvements are achieved compared to always using SM with 4 transmitters. Generally the simulations suggest that, for mobile optical receivers angular diversity detectors can perform better than vertical oriented receivers. Also ASM improves the SM performance in mobile receivers.

Future work will focus on the effect of reflected optical paths on conditions for the scenarios discussed in this paper. It is expected that, the diffuse channels model will provide further throughput improvement due to the fact that receivers can exploit both LOS paths and reflected paths.

\section{ACKNOWLEDGEMENT}

H. Haas acknowledges support from the Engineering and Physical Sciences Research Council (EPSRC) under Established Career Fellowship grant EP/K008757/1

\section{REFERENCES}

[1] R. Mesleh and H. Haas, "Indoor Optical Wireless Communications; Potential and state-of-art" IEEE Communication magazine, Vol. 49, pp. 56-62, September. 2011.

[2] L. Zeng and D. O'Brien, "High data rate multiple input multiple output (MIMO) optical wireless communications using white led lighting" IEEE Journal on Selected Areas in Communications, Vol 27, no 9 pp. 1654-1662, December. 2009.

[3] R. Mesleh et al. "Indoor MIMO Optical Wireless Communication Using Spatial Modulation" IEEE International Conference on Communications (ICC), Cape Town, South Africa. pp. 1-5, May. 2010.

[4] D. O'Brien, "Multi-input multi-output (MIMO) indoor optical wireless communications" Conference Record of the Forty-Third Asilomar Conference on Signals, Systems and Computers, Pacific Grove, CA. pp. 1636-1639, Nov. 2009.

[5] C. Pei et al. "Design of Indoor Optical Wireless Collaborative Cellular System" IEEE Wireless Communications and Signal Processing, Nanjing, Vol 1, pp. 1-6, November, 2011.

[6] P. Fahamuel et al. "Study, analysis and application of optical OFDM, single carrier (SC) and MIMO in intensity modulation direct detection (IM/DD)" IET Intelligent Signal Processing Conference 2013, London, pp. 1-6, December, 2013 .

[7] Z. Cheng, N. Serafimovski and H. Haas, "Angle diversity for an Indoor cellular Visible Light Communication System" VTC Spiring 2014, Seoul, May 2014.

[8] T. Fath and H. Haas, "Performance Comparison of MIMO Techniques for Optical Wireless Communications in Indoor Environments" IEEE Transactions on Communications, Vol 61, no 2. pp. 733-742, February. 2013.

[9] S. Grant et al. "Per Antenna-Rate-Control (PARC) in Frequency selective Fading with SIC-GRAKE Receiver", IEEE Vehicular Technology Conference, Vol 7, pp. 26-29, September 2004

[10] R. Masleh et al. "Spatial Modulation-A New Low Complexity Spectral Efficiency Enhancing Technique" First International Conference on Communications and Networking in China, 2006. ChinaCom '06, Beijing, pp. 1-5, October, 2006

[11] R.Mesleh et al. "Spatial Modulation" IEEE Vehicular Technology Conference, Vol 57, no 4, pp. 2228-2241, July, 2008.

[12] R.Mesleh et al. "Trellis coded spatial modulation" IEEE Transactions on Communications, Vol 9, no 7, pp. 2349-2361, July, 2010.

[13] M. Christiansen and K. Duffy, "Brute force searching, the typical set and Guesswork" IEEE Information Theory Proceedings, Instanbul, pp. 1257-1261, July, 2013 


\title{
Performance analysis of indoor diffuse VLC MIMO channels using Angular Diversity Detectors
}

Paul Fahamuel, Student Member, IEEE, John Thompson, Member, IEEE,

and Harald Haas, Member, IEEE

\begin{abstract}
We consider specular and diffuse reflection models for indoor visible light communications (VLC) using a mobile receiver with angular diversity detectors in multiple input multiple output (MIMO) channels. We aim to improve the MIMO throughput compared to vertically oriented detectors by exploiting multipath reflections from different surfaces in the room. We then evaluate data throughput across multiple locations in the small room by using repetition coding, spatial multiplexing and spatial modulation approaches. In spatial modulation, we also propose a novel approach called adaptive spatial modulation (ASM). This makes use of channel matrix rank information to decide which TX/RX setup to be used, and is developed to cope with rank deficient channels. In a scenario where the receiver is moving, channel gains are weak in some locations due to the lack of line of sight (LOS) propagation between transmitters and receivers. This effect is mitigated by employing adaptive modulation and coding (AMC) together with per antenna rate control (PARC). We then compare the throughput for LOS only channels against LOS with specular or diffuse reflection conditions, for both vertical and angular
\end{abstract}

\footnotetext{
This work was presented in part at the IEEE Global Communications Conference (GLOBECOM 2014), Houston, Texas, USA.

The work was supported in part by the Dar es salaam Institute of Technology (DIT) under the Ministry of Education and Vocation Training (Tanzania Government) through Science and Technology for Higher Education Project (SHTEP, 05/2008-06/2013).

Part of research was supported by the university of Edinburgh, Institute for Digital communications.

P. Fahamuel is with the Dar es salaam Institute of Technology (DIT), Department of Electronics and Telecommunications Engineering, Tanzania. P. o Box 2958, Dar es salaam and with the University of Edinburgh, Institute for Digital Communications, the Kings Buildings, Edinburgh EH9 3JL, UK (e-mail: p.mmbaga@ed.ac.uk, pfahamuel@dit.ac.tz).

J. Thompson and H. Haas are with the University of Edinburgh, Institute for Digital Communications, the Kings Buildings, Edinburgh EH9 3JL, UK (e-mails:\{john.thompson, h.haas\}@ed.ac.uk) 
oriented receivers. The results show that exploiting specular and diffuse reflections provide significant improvements in link performance.

\section{INTRODUCTION}

With the development of wireless communications applications, there is a rapid rise in of data demand, while the available radio frequency (RF) spectrum cannot meet this growth and hence becomes the limiting factor for achieving higher transmission rates [1]. The spectrum ranging from $10 \mu \mathrm{m}$ (infra-red) to $10 \mathrm{~nm}$ (ultraviolet) including visible light offers nearly limitless bandwidth which may be utilized for communications such as wireless local area networks (WLAN). In optical wireless communications, the light emitting diode transmitter modulates data and transforms the electrical signal to an optical signal while the photo-diode receiver converts the incoming optical signal into an electrical current for data processing. Optical wireless communications (OWC) therefore promises to be a low cost and high throughput alternative to RF communications. With the development of solid-state lighting, white light emitting diodes (LEDs) will replace existing conventional light bulbs so communications and illumination can take place simultaneously, hence saving power [2]. It is also safe to use in places where RF signals are not permitted e.g. hospitals, chemical plants and gas/petrol filling stations. Visible light communications is cheap because of the low cost and reliability of light sources and receivers.

Indoor OWC systems can be classified into diffuse and line of sight (LOS) systems. In LOS systems high data rates of the order of gigabits per second can be achieved [1], [3] but these systems are vulnerable to obstacles (shadowing) because of their directionality. In diffuse systems, several propagations paths exist from the LED to photo-diode (PD) which makes the system robust to shadowing [4], however the path loss can be higher than for LOS systems and multipath creates inter-symbol interference (ISI) for the case of large area indoor environments [2], [5]-[7]. The simultaneous use of multiple transmitters and receivers e.g. OWC multiple input multiple output (MIMO) can enhance the overall system performance and spectral efficiency as well as reducing the bit error ratio (BER) performance of a communication system [2]. MIMO systems can realize higher speed transmission without increasing the transmit power or the bandwidth.

Kahn and Barry gives more details about wireless infrared communication in [11]. Here the use of infrared radiation as a medium for high-speed, short range OWC is discussed, advantages and drawbacks are compared. MIMO techniques have been applied for OWC and data transmission 
to a limited extent. High data rate MIMO optical wireless communications using white LEDs were proposed in [2], where a fixed receiver with an imaging lens was used. It was shown that the imaging lens and detector array size are physically large and may not be practical for some applications. Another indoor OWC MIMO system with an imaging receiver was proposed in [8], here the diffuse environment experiments were performed. The system shows error-free operation at $2 \mathrm{Mbit} / \mathrm{s} / \mathrm{spatial}$ channel at the center of the coverage area, with worse performance away from the center. In [9] results from several indoor OWC MIMO experiments were reported, a four channel MIMO system that uses white LEDs for communications was described as well as experiments in a diffuse environment using infra-red sources. An omnidirectional multibeam transmitter in [10] was proposed to improve transmission coverage and overcome shadowing, in this paper a multibeam hemispherical receiver structure was found to reduce multipath effects. The simulation results showed significant reduction of the BER making the system suitable for high bit rate applications. An indoor optical wireless collaborative cellular system was proposed in [12]. In the paper, base stations and portable devices were designed, presented and analysed. Base stations (BS) providing multiple cells were proposed to cover a wide area, however the MIMO techniques did not perform properly at all the receiver positions due to channel symmetry effects.

Research development on overcoming channel correlation and inter-symbol interference yielded the method proposed in [13]-[15]. Here indoor OWC MIMO using spatial modulation (SM) was suggested and implemented, where unlike other MIMO techniques, only one transmitter is active at any given time instant. The active transmitter radiates at a certain intensity level and all other transmitters are turned off. In SM, it was found that reducing both the distance between transmitters (TX) and receivers (RX) and the transmitter emerging angle (the angle between TX axis and the straight line to the RX) resulted in lower correlation and hence higher data throughput. A hemispherical lens based imaging receiver for OWC MIMO was described in [16], [17]. These papers presented a novel imaging MIMO optical wireless system which uses a hemispherical lens in the receiver, this system has both a wide field of view (FOV) and showed significant spatial diversity. In [18], a performance comparison of OWC MIMO Techniques in indoor environments was provided between repetition coding (RC), SM and spatial multiplexing. The results show that spatial multiplexing (SMP) improves the spectral efficiency 
when there is low channel correlation. It was also shown that SM is competitive at low spectral efficiency while SMP performs better in high spectral efficiency where SM needs a very large signal constellation size to match SMP. Also it is more robust to channel correlation. RC was found to be insensitive to different transmitter-receiver alignments but it needs a large signal constellation size to provide high data rates. The practical MIMO capacity for indoor optical wireless communication with white LEDs was presented in [19]. In this paper the communication scenario assumed an angle diversity receiver with multiple detector elements where each element has an independent concentrator/lens. A significant capacity improvement was achieved in such a system.

In [20], the receiver performance improvement utilizing diversity in MIMO VLC with an imaging receiver was presented. It was shown that by employing maximal ratial combining at the receiver performance can be improved significantly, however the receiver may be impractical due to the size and complexity of imaging devices. Wang and Armstrong in [21], [22] analysed the performance of an indoor MIMO optical wireless system with a linear receiver. The receiver used an array of prisms to form channel matrices that can achieve angular diversity within a compact receiver structure. It was shown that full column rank can be achieved by the proposed receiver over an entire room. In [23] Wang and Chi experimentally demonstrated a $2 \times 2$ nonimaging MIMO VLC system that is capable to deliver $500 \mathrm{Mb} / \mathrm{s}$. However it was concluded that the large size of the lens and the detectors required are not practical. In [24], the receiver with angular diversity detectors was proposed and found to overcome the channel rank deficiency which occurs in areas away from the center of the room.

In these papers, the VLC coverage is among the major drawbacks. In most papers movement of the receiver to different locations to asses the impact on channel correlation and hence spectral efficiency was not covered. Further the effect of diffuse channel reflections on MIMO was not discussed. The present paper addresses these issues and contains the following novel contributions:

- Propose a novel adaptive apatial modulation(ASM) method to tackle reduced channel rank.

- First evaluation of whole room MIMO performance using vertical and angular diversity receivers.

- Novel study of the impact of specular and diffuse reflections on MIMO performance. 
We use a MIMO system taking into account line of sight (LOS) propagation, The Lambertian specular reflections model and the Lambert-Phong diffuse reflection model [4] for both vertical and angular oriented receiver detectors. We then provide performance statistics for MIMO methods operating over many room locations using adaptive modulation and coding (AMC), ASM, and per antenna rate control (PARC) [25]. We also consider RX performance improvement by application of angular diversity techniques.

The rest of the paper will be as follows. Section II presents the system models. Section III explains the MIMO techniques used in this paper. Section IV describes the evaluation of the system throughput. Section V shows the simulation parameters and the different simulation scenarios, along with the results and discussion. Section VI concludes the paper.

\section{SYSTEM MODELS}

We consider visible light communications (VLC) MIMO transmission and take into account both specular and diffuse reflections where intensity modulation (IM) and direct detection (DD) of the optical carrier using an incoherent light source is employed. The system consists of $N_{t}$ transmitters and $N_{r}$ photo-detectors at the receiver side. The size $N_{r}$ received signal vector $\mathbf{y}$ is

$$
\mathbf{y}=\mathbf{H s}+\mathbf{n}
$$

Where $\mathbf{H}$ is the $N_{t} \times N_{r}$ channel matrix and $\mathbf{s}$ is the transmitted signal vector which is transmitted at a given time and is defined as follows: $\mathbf{s}=\left[\begin{array}{lll}s_{1} & \ldots \ldots s_{N t}\end{array}\right]^{T}$ with [. $]^{T}$ being the transpose operator and $s_{n}$ denoting the signal transmitted by $n^{\text {th }}$ LED. The sum of the ambient light shot noise and thermal noise is denoted by $N_{r} \times 1$ vector $\mathbf{n}$ which is assumed to be real valued additive white Gaussian noise (AWGN) with zero mean and variance:

$$
\delta^{2}=\delta_{\text {shot }}^{2}+\delta_{\text {thermal }}^{2}
$$

Where $\delta^{2}$ shot is the shot noise variance and $\delta^{2}$ thermal is the thermal noise variance as calculated in equations (7) and (10) of [7] respectively. Thus the noise power is given by $\delta^{2}=N_{0} B$, where $N_{0}$ is the noise power spectral density and $B$ is communication bandwidth.

This paper assumes an optical wireless LOS link operating in a room with reflection characteristics (Fig. 1). There are two types of reflections considered in this paper. The first type is a single bounce specular reflection which is modelled by an image transmitter $T X_{n}^{\prime}$ to the receiver (Fig. 1. a). The reflections cause the signal to be attenuated by the surface reflection coefficient 


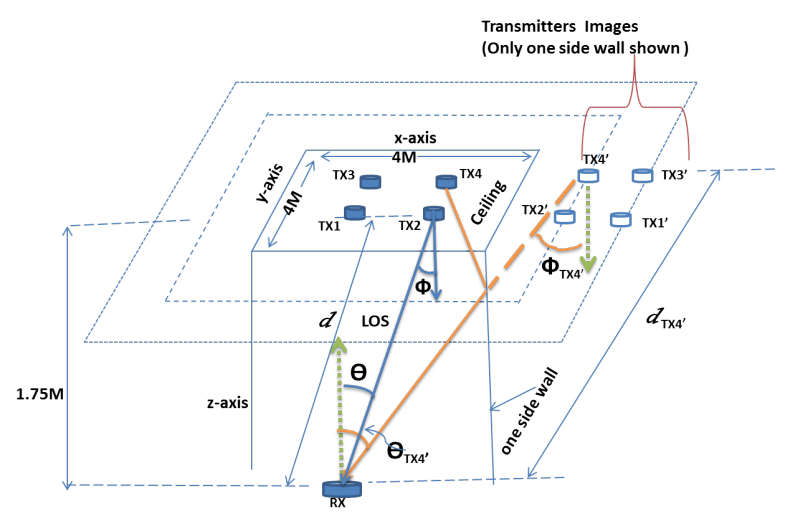

(a)
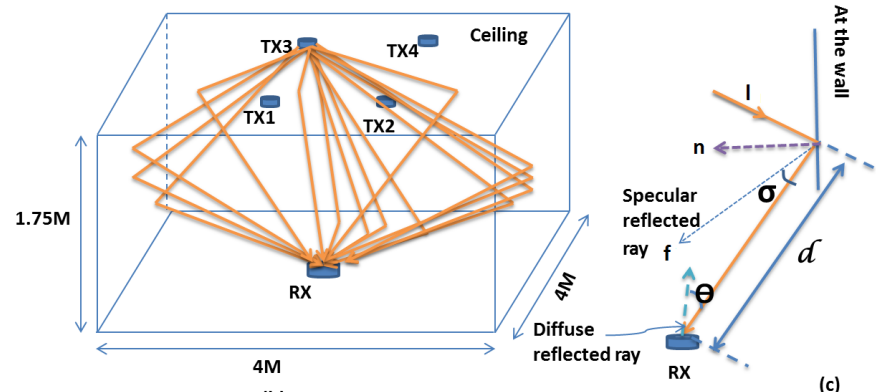

(b)

(c)

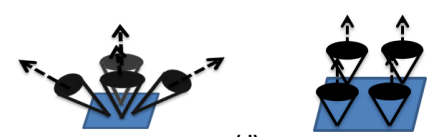

(d)

Fig. 1. Transmitter and receiver geometry used for channel coefficient calculations (a) Specular reflection model (b) Diffuse reflection model (c) Diffuse reflection-wall geometry (d) Receiver with inclined and vertical axis detectors

$\alpha$. The second type is a diffuse reflection where all the rays bouncing from the reflecting surface is scattered into different directions of the room (Fig. 1. b). The incidence power from the wall is assumed to be a fraction of the total transmitted power wich is dictated by the number of rays re-radiated.

The LOS $N_{r} \times N_{t}$ channel matrix $\mathbf{H}$ for each room coordinate $(x, y, z)$ in the room is given by 


$$
\mathbf{H}(x, y, z)_{L O S}=\left(\begin{array}{ccccc}
h_{11}(x, y, z) & h_{12}(x, y, z) & . & \cdot & h_{1 N t}(x, y, z) \\
h_{21}(x, y, z) & h_{22}(x, y, z) & & \cdot \\
\cdot & & \cdot & \cdot \\
\cdot & & & \cdot & \cdot \\
h_{N r 1}(x, y, z) & . & . & \cdot & h_{N r N t}(x, y, z)
\end{array}\right)
$$

where $h_{n_{r} n_{t}}$ represents the channel transfer function of the wireless link between transmitter $n_{t}$ and receiver $n_{r}$. For the specular reflection model $\left(\mathrm{S}_{\mathrm{p}}\right)$ (Fig. 1 (a)) and the diffuse reflection model $\left(\mathrm{D}_{\mathrm{f}}\right)$ (Fig. 1 (b)), the total channel gain at the receiver detector $n_{r}$ from transmitter $n_{t}$ is

$$
h_{n_{r} n_{t}\left(S_{p} / D_{f}\right)}=\sum_{i=1}^{N_{T X_{n}}} h_{n_{r} n_{t i}}
$$

where for $\mathrm{S}_{\mathrm{p}}, N_{T X n}$ is the number of transmitter images received at detector $n_{r}$ caused by transmitter $n_{t}$ and for $\mathrm{D}_{\mathrm{f}} N_{T X n}$ is the number of scattered light rays received from transmitter $n_{t}$. Therefore, when all LOS, SR and DR rays are considered, the channel gain at the individual receiver detector $n_{r}$ will be

$$
h_{n_{r} n_{t(T o t a l)}}=h_{n_{r} n_{t(L O S)}}+h_{n_{r} n_{t\left(S_{p} / D_{f}\right)}}
$$

So, the overall $N_{r} \times N_{t}$ channel matrix $\mathbf{H}$ for each room coordinate $(x, y, z)$ in the room will be represented by substituting (5) into (3). The path difference between the multiple transmitterreceiver links is very small, on the order of few $\mathrm{cm}$ as shown in Fig. 1. We assume for simplicity that the communications channel bandwidth is much less than the inverse of the delay spread, so the channel is not frequency selective.

By using the Lambert-Phong method [4] the diffuse paths are assumed to be scattered paths reradiated from the wall to the receiver after being attenuated by the surface reflection coefficient $\alpha$. As illustrated in Fig. 1 (a), $\phi_{T X_{n}^{\prime}}$ is the angle of emergence with respect to the image of transmitter $T X_{n}$ axis, $\theta_{T X_{n}}$ is the angle of incidence with respect to the receiver detector axis and $d_{T X_{n}}{ }^{\prime}$ is the distance between the image transmitter $T X_{n}^{\prime}$ and receiver. Also as illustrated in Fig. 1 (c), $\sigma$ is the angle of emergence with respect to the direction of specular reflected ray axis $\mathbf{f}$ which is a directional vector with coordinates $(x, y, z), \theta$ is the angle of incidence with respect to the receiver detector axis and $d$ is the distance between the reflecting point and the receiver. The system transmitters are fixed at the following ceiling coordinates: 
$T X_{1}[1.9 \mathrm{~m}, 1.9 \mathrm{~m}, 2.75 \mathrm{~m}], T X_{2}[2.5 \mathrm{~m}, 1.9 \mathrm{~m}, 2.75 \mathrm{~m}], T X_{3}[1.9 \mathrm{~m}, 2.5 \mathrm{~m}, 2.75 \mathrm{~m}]$, and $T X_{4}[2.5 \mathrm{~m}, 2.5 \mathrm{~m}, 2.75 \mathrm{~m}]$.

The transmitters are arranged in a square in the middle of the ceiling and the antenna side separation is $0.6 \mathrm{~m}$ as this choice was found to reduce channel correlation in [24].

Consider a LOS optical system propagation path in Fig. 1, the channel gain from the transmitter to the receiver is given by [18]:

$$
h=\left\{\begin{array}{cc}
\frac{A(k+1) \cos ^{k}(\phi) \cos (\theta)}{2 \pi d^{2}}, & 0 \leqslant \theta \leqslant \varphi_{1 / 2} \\
0, & \theta>\varphi_{1 / 2}
\end{array}\right.
$$

where $k=\frac{-\ln (2)}{\ln \left(\cos \left(\Phi_{1 / 2}\right)\right)}$.

The scalar $A$ is the collection area of the receiver $n_{r}, \Phi_{1 / 2}$ is the transmitter semi-angle (at half power), which is assumed to be $45^{\circ}$. The scalar $\varphi_{1 / 2}$ is the Field of View (FOV) semi angle of the receiver which is also assumed to be $45^{\circ}$. In [18] $\Phi_{1 / 2}$ and $\varphi_{1 / 2}$ were assumed to be both $15^{\circ}$, but for this setup where the receiver is moving around the room, it is difficult to achieve LOS channel conditions in many locations when a narrower half angle is used. We set $h_{n_{r} n_{t(L O S)}}=0$, when a receiver and transmitter are not in each others' FOV. Image transmitters in the specular reflection model use the same equation (6) with their respective angles, then the gain is multiplied by the reflection attenuation $\alpha$ to yield the corresponding channel coefficient

$$
h=\alpha \frac{A(k+1)}{2 \pi d_{T X_{n}}{ }^{2}} \cos ^{k}\left(\phi_{T X_{n}}^{\prime}\right) \cos \left(\theta_{T X_{n}}{ }^{\prime}\right)
$$

For the diffuse reflection case the Lambert-Phong model is used [4]. We define a reflection intensity scattering using a generalized Lambert radiation pattern

$$
P_{\text {Wall }}=\alpha \frac{P_{i}\left(m_{s}+1\right)}{2 \pi} \cos ^{m_{s}}(\sigma)
$$

where $P_{i}$ is the incident normalized unit power arriving at the wall, $P_{W a l l}$ is the reflection intensity from the reflecting surface, $m_{s}$ is the smoothness of the reflecting material and $\sigma$ is the randomly generated parameter which represents the angle between specular reflected rays and the diffuse reflected ray (Fig. 1. c). To calculate the specular reflected ray vector $\mathbf{f}$ we make use of the normal vector $\mathbf{n}$ to the wall and the vector $\mathbf{I}$ which is the line connecting transmitter and the wall (see Fig. 1. c): 


$$
\mathbf{f}=(\mathbf{2 n} \bullet \mathbf{l}) \mathbf{n}-\mathbf{l}
$$

where $\mathbf{f}, \mathbf{n}$ and $\mathbf{l}$ are three dimensional vectors with components (x, y, z). The dot mark in (9) denotes the vector dot product. As shown in Fig. 1. c, once f and $\sigma$ are known then all the diffusely reflected paths can be generated. So, the channel gain for one reflected ray from the wall to the receiver is expressed as

$$
h\left(\sigma, m_{s}\right)=\left\{\begin{array}{cc}
\alpha \frac{A\left(m_{s}+1\right)}{2 \pi d^{2}} \cos ^{m_{s}}(\sigma) \cos (\theta), & 0 \leqslant \theta \leqslant \varphi_{1 / 2} \\
0, & \theta>\varphi_{1 / 2}
\end{array}\right.
$$

and the incident optical power to the receiver, see [7], [26], [27] can be calculated by

$$
P_{i r}=P_{W a l l} \frac{A \cos (\theta)}{d^{2}}=h\left(\sigma, m_{s}\right) P_{i}
$$

We now briefly describe the technique used for configuring the inclined optical detectors of the receiver. The left side of Fig.1(d) shows the receiver with inclined detectors. To achieve this setup the detector axis vector $\mathbf{z}$ which is inclined at an elevation angle $45^{\circ}$ is transformed around the $z$ axis (vertical axis) using the transformation matrix as in [24]. The orientation angle of the receiver in the $x-y$ plane is assumed to be random, which is likely to be the case in practice as different users will hold their devices in different orientations. The orientation of the receiver is given by

$$
\begin{array}{ccc}
\hat{\mathbf{z}}= & \mathbf{R}_{\mathbf{z}}(\vartheta+\omega) & \times \mathbf{z} \\
\left(\begin{array}{l}
\hat{x} \\
\hat{y} \\
\hat{z}
\end{array}\right)=\left(\begin{array}{ccc}
\cos (\vartheta+\omega) & -\sin (\vartheta+\omega) & 0 \\
\sin (\vartheta+\omega) & \cos (\vartheta+\omega) & 0 \\
0 & 0 & 1
\end{array}\right)\left(\begin{array}{l}
x \\
y \\
z
\end{array}\right)
\end{array}
$$

where $\hat{\mathbf{z}}$ is the transformed vector, $\vartheta$ presents the transformation angle around the $z$ axis to form the detectors' axis vectors pointing at azimuth angles $0^{\circ}, 90^{\circ} 180^{\circ}$ and $270^{\circ}$ with an additional random rotation angle $\omega$ which is uniformly distributed between $0^{\circ}$ to $360^{\circ}$. The matrix $\mathbf{R}_{\mathbf{z}}$ is the transformation matrix with respect to the $z$ axis. Since any variation of the detector's axis affects the angle of incidence $\theta$ as in (6) and (10), each detector elevation angle is varied from 
$45^{\circ}$ to $90\left(0^{\circ}\right.$ from vertical $)$ to find the elevation angle that will maximize the rank of channel matrix $\mathbf{H}$. Therefore a single selected elevation angle is used for the inclined axis detector for system performance evaluation at all locations in the room.

\section{MIMO TECHNIQUES}

In this paper four different MIMO techniques are used; repetition coding (RC), spatial modulation (SM), adaptive spatial modulation (ASM) and spatial multiplexing (SMP) as in [24]. In contrast to [2], [9], [13] where fixed receivers are used, in this paper we consider that mobile receiver is able to move freely around the room as in [24]. To overcome the resulting SNR variations we employ adaptive modulation with per antenna rate control (PARC) [25] in SMP and adaptive modulation in SM and RC. In these techniques, the modulation $M$-level is chosen and updated in each transmit time and at each location depending on the current channel conditions. We assume that all considered MIMO techniques use maximum likelihood (ML) [18] detection at the receiver with perfect knowledge of the channel and ideal time synchronisation except in SMP where the zero forcing (ZF) detection method is used to reduce receiver complexity when using PARC. Therefore in RC, SM and ASM the decoder selects a constellation vector $\hat{\mathbf{s}}$ which minimizes the Euclidean distance between the actual received signal $\mathbf{y}$ and all the possible signal vectors leading to

$$
\hat{\mathbf{s}}=\arg \underset{\mathbf{s}}{\max } p_{\mathbf{y}}(\mathbf{y} \mid \mathbf{s}, \mathbf{H})=\underset{\mathbf{s}}{\operatorname{argmin}}\|\mathbf{y}-\mathbf{H s}\|_{F}^{2}
$$

where $p_{\mathbf{y}}$ is the probability density function of $\mathbf{y}$ conditioned on $\mathbf{s}$ and $\mathbf{H}$. The notation $\|\cdot\|_{F}$ indicates the Frobenius norm.

\section{A. Repetition coding (RC)}

The first technique used is RC which simultaneously emits the same signal from all transmitters. Therefore the condition $s_{1}=s_{2}=\ldots . . s_{N t}$ holds [18]. In RC, the light intensities arising from the several transmitters constructively add up at the receiver side. In this paper, unipolar $M$-level pulse amplitude modulation ( $M$-PAM) is considered together with RC, where $M$ denotes the signal constellation size. Therefore $M$-PAM achieves a spectral efficiency of $\log _{2}(M)$ bit/s/Hz. PAM is more bandwidth efficient compared to other pulse modulation techniques such as pulse-width modulation (PWM), on-off keying (OOK) and pulse -position modulation (PPM). [18]. Moreover PAM has been shown to outperform direct current biased optical OFDM (DCO-OFDM) because 
the later requires a high constant DC bias to make the bipolar OFDM waveform non-negative [5], [6]. We employ rectangular pulse shapes with $M$-PAM, so the intensity level emitted by the Light Emitting Diode (LED) is given by

$$
I_{m}^{P A M}=\frac{2 I}{M-1} m, \text { for } m=0,1, \ldots \ldots . .(M-1)
$$

where $I$ is the mean optical power emitted. The Bit Error Rate (BER) for unipolar $M$-PAM can be expressed by

$$
\mathrm{BER}_{\mathrm{PAM}} \geqslant \frac{2(M-1)}{M \log _{2}(M)} Q\left(\frac{1}{M-1} \sqrt{\frac{E_{R X}}{N_{0}}}\right)
$$

where:

$$
Q(a)=\frac{1}{\sqrt{2 \pi}} \int_{a}^{+\infty} \exp \left(\frac{-t^{2}}{2}\right) d t
$$

is the $Q$ function [18] and $E_{R X}$ is the received electrical energy. The BER of $M$-PAM given in (15) can be generalized for the $N_{r} \times N_{t}$ scenario and the resulting BER is given by:

$$
\mathrm{BER}_{\mathrm{RC}} \geq \frac{2(M-1)}{M \log _{2}(M)} Q\left(\frac{1}{M-1} \sqrt{\frac{E_{s}}{N_{0} N_{t}^{2}} \sum_{n_{r}=1}^{N_{r}}\left(\sum_{n_{t}=1}^{N_{t}} h_{n_{r} n_{t}}\right)^{2}}\right)
$$

Where, $E_{s}=(\varrho I)^{2} T_{s}$ denotes the mean emitted electrical energy of the intensity modulated optical signals. The symbol $\varrho$ represents the optical to electrical conversion coefficient, it is assumed that, $\varrho=1 \Lambda / \mathrm{W}$. The scalar $T_{s}$ denotes the symbol duration in seconds and $\Lambda$ is the unit current in amperes.

B. Spatial Modulation (SM)

The second technique used in this paper is SM, which combines MIMO and digital modulation as proposed in [28] and further investigated in [29], [30] and also applied in [14], [15]. In SM the conventional constellation diagram is extended to an additional dimension namely, the spatial dimension. Specifically, the LED index is used to communicate data bits to the receiver. Each transmitting LED is assigned a unique binary sequence (the spatial symbol). A transmitter is only activated when the random spatial symbol to be transmitted matches the specified transmitter index. Thus, only one transmitter is activated for any PAM symbol transmission so only one element of the signal vector $\mathbf{s}$ to be transmitted is non-zero. SM can provide an enhanced spectral 


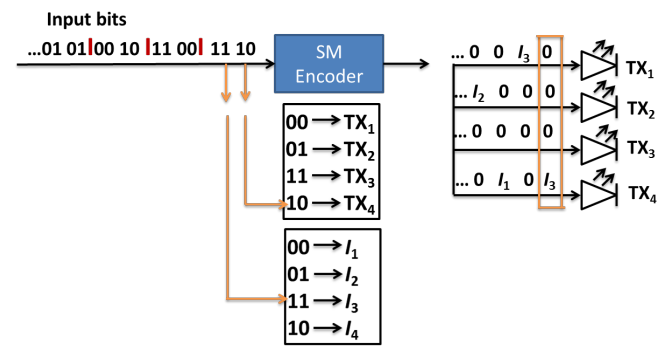

Fig. 2. Illustration of SM operation with $N_{t}=4$ and $M=4$. The first two bits in the block of four bits determine the PAM symbol and second two bits determine the active LED [15]

efficiency of $\log _{2}\left(N_{t}\right)+\log _{2}(M)$ bit/s/Hz, see in [13]-[15], [26], [28], [30], [33]-[36]. Signals with intensity $I_{m}=0$ cannot be used for the signal modulation of SM, because in this case no transmitter would be active and the spatial information information would be lost [18]. Therefore, the intensities of conventional PAM given in (7) have to be modified to be compatible with SM leading to:

$$
I_{m}^{\mathrm{SM}}=\frac{2 I}{M+1} m, \text { for } m=1, \ldots \ldots .(M)
$$

The BER expression for SM is given by:

$$
\begin{array}{r}
\mathrm{BER}_{\mathrm{SM}} \leq \frac{1}{M N_{t} \log _{2}\left(M N_{t}\right)} \sum_{m^{(1)}=1}^{M} \sum_{n_{t}^{(1)}=1}^{N_{t}} \sum_{m^{(2)}=1}^{M} \sum_{n_{t}^{(2)}=1}^{N_{t}} \mathrm{~d}_{\mathrm{H}}\left(b_{m^{(1)} n_{t}^{(1)}}, b_{m^{(2)} n_{t}^{(2)}}\right) \\
Q\left(\sqrt{\frac{E_{s}}{4 N_{0}} \sum_{n_{r}=1}^{N_{r}}\left|I_{m^{(2)}}^{\mathrm{SM}} h_{n_{r} n_{t}^{(2)}}-I_{m^{(1)}}^{\mathrm{SM}} h_{n_{r} n_{t}^{(1)}}\right|^{2}}\right)
\end{array}
$$

Where, $\mathrm{d}_{\mathrm{H}}\left(b_{m^{(1)}}, b_{m^{(2)}}\right)$ denotes the Hamming distance of two bit assignments $b_{m^{(1)}}$ and $b_{m^{(2)}}$ of the signal vectors $\mathbf{s}_{m^{(1)}}$ at the transmitter and signal $\mathbf{s}_{m^{(2)}}$ at the receiver.

\section{Adaptive Spatial Modulation (ASM)}

The third technique is ASM which is a modified form of spatial modulation (SM). ASM is proposed in this paper to cope with rank deficient channels due to the fact that, when the receiver moves around the room the channel matrix $\mathbf{H}$ is not full rank (4) in many locations. If the channel matrix rank is reduced, the receiver can not easily distinguish all the transmitting 
LEDs. By using antenna selection techniques, the ASM receiver checks the rank of the channel matrix and decides which TX/RX setup to be used.

The authors in [31], [32], [34] proposed some different techniques for antenna selection. This paper will discus the methods proposed in [34] which is relevant to the technique proposed in this paper. In [34] they use two methods which reduce the order of complexity (number of times the optimization metric is evaluated). The first method was a Euclidean distance optimized antenna selection (EDAS) which chooses the specific antenna set that maximizes the minimum Euclidean distance among all the possible transmit vectors. The second one was capacity optimized antenna selection (COAS) which uses the bounded system capacity to chooses the set of antennas corresponding to the largest channel norms out of number of transmitting antennas. In this paper a similar method to COAS is used but with the difference that the system capacity is not used as the metric as it is not applicable to positive real channels that arise in OWC. Instead the channel matrix rank is used here to decide the number of antennas to be active, thereafter the largest channel norms will be used to decide the set of antennas to be used. The following three steps are involved.

- If $\operatorname{rank}(\mathbf{H})=4$, we use four transmitter Adaptive Modulation SM for transmission and throughput calculations.

- If $\operatorname{rank}(\mathbf{H})=2$ or 3, we employ 2TX Adaptive Modulation SM. To identify which two transmitters are to be activated we check the $\mathbf{H}$ matrix using the norm based method (21). Assume $\mathbf{q}_{\mathbf{n}_{\mathbf{t}}}$ is the column of $\mathbf{H}$ as follows:

$$
\mathbf{q}_{\mathbf{n}_{\mathbf{t}}}=\left(\begin{array}{c}
h_{1} n_{t} \\
\cdot \\
\cdot \\
\cdot \\
h_{N r} n_{t}
\end{array}\right), \quad n_{t}=1, \ldots \ldots, N_{t}
$$

Then we select the two highest norm of sum of each $\mathbf{q}_{\mathbf{n}_{\mathbf{t}}}$ values such that the selected two norms will present the column of transmitters $T X_{1}$ and $T X_{2}$ :

$$
\operatorname{Norm}_{n_{t}}=\sum_{n_{r}=1}^{N_{r}}\left|h_{n_{r} n_{t}}\right|, n_{t}=1 \ldots . . N_{t} .
$$

then we sort the norms in decreasing order so that the first two largest norms identify 
transmitters to be selected.

- If $\operatorname{rank}(\mathbf{H})=1$, we use RC with Adaptive Modulation.

If $\Omega$ denotes the number of selected antennas, the BER of ASM is approximated using the joint BER evaluation method. This uses both the SM and the RC BERs as used in [18], which are jointly evaluated as follows :

$$
\mathrm{BER}_{\mathrm{ASM}} \leq\left\{\begin{array}{cc}
\frac{1}{M \Omega \log _{2}(M \Omega)} \sum_{m^{(1)}=1}^{M} \sum_{n_{t}^{(1)}=1}^{\Omega} \sum_{m^{(2)}=1}^{M} \sum_{n_{t}^{(2)}=1}^{\Omega} \mathrm{d}_{\mathrm{H}}\left(b_{m^{(1)} n_{t}^{(1)}}, b_{m^{(2)} n_{t}^{(2)}}\right) . & \\
Q\left(\sqrt{\frac{\varrho^{2} T_{s}}{4 N_{0}} \sum_{n_{r}=1}^{N_{r}}\left|I_{m^{(2)}}^{\mathrm{SM}} h_{n_{r} n_{t}^{\prime}}^{\prime}-I_{m^{(1)}}^{\mathrm{SM}} h_{n_{r} n_{t}^{(1)}}^{\prime}\right|^{2}}\right), & 2 \leq \Omega \leq 4 \\
\frac{2(M-1)}{M \log _{2}(M)} Q\left(\frac{1}{M-1} \sqrt{\frac{E_{s}}{N_{0} N_{t}^{2}} \sum_{n_{r}=1}^{N_{r}}\left(\sum_{n_{t}=1}^{N_{t}} h_{n_{r} n_{t}}\right)^{2}}\right), & \Omega=1
\end{array}\right.
$$

In this equation $h_{n_{r} n_{t}}^{\prime}$ denotes the channel coefficients of the selected antennas.

\section{Spatial Multiplexing (SMP)}

The final MIMO technique is SMP. In SMP independent data streams are simultaneously transmitted from all the transmitters. Since ZF is used to estimate the transmitted symbol in SMP, the following equation is used to obtain the estimate value of $\mathbf{s}$ :

Consider equation (1),

$$
\hat{\mathbf{s}}=\mathbf{W} \cdot \mathbf{y}
$$

where, $\mathbf{W}$ denotes the Pseudo-inverse of channel matrix $\mathbf{H}$ which is given by:

$$
\mathbf{W}=\left(\mathbf{H}^{T} \mathbf{H}\right)^{-1} \mathbf{H}^{T}
$$

SMP provides a maximum spectral efficiency of $N_{t} \log _{2}(M)$ bit/s/Hz. As for RC, PAM is used with SMP and equally distributes the optical power across all emitters to ensure that both schemes use the same transmit power. The BER for SMP is given as:

$$
\mathrm{BER}_{\mathrm{SMP}} \leq \frac{1}{N_{t}} \sum_{i=1}^{N_{t}} \frac{2\left(M_{i}-1\right)}{M_{i} \log _{2}\left(M_{i}\right)} Q\left(\frac{1}{M_{i}-1} \sqrt{\frac{E_{s}}{N_{0} N_{t}^{2}\left\|\mathbf{W}_{i}\right\|^{2}}}\right)
$$

where, $\mathbf{W}_{i}$ denotes $i^{\text {th }}$ row of pseudo-inverse of channel matrix $\mathbf{H}, M_{i}$ is the $i^{\text {th }}$ selected modulation level for transmitter LED $i$. 
IV. SyStem ThroughPUT EVALUATION

For the RC techniques we use the BER Expression described in equation (17) and calculate throughput given by [7]:

$$
T_{h(\mathrm{SISO})}=R(1-\gamma) \mathrm{bps} / \mathrm{Hz}
$$

Where $R$ is the maximum rate of the scheme and $\gamma$ is the packet error probability which is given by [7]:

$$
\gamma=1-(1-B E R)^{N_{b}}
$$

Where, $N_{b}$ is the number of bits in one packet. Equation (26) is computed for all modulation sizes $M$ which yield a BER less than $10^{-2}$ (see Fig. 3 of [24]). Then, the highest throughput determines the modulation scheme that is selected. A BER greater values than $10^{-2}$ is ignored since will not give a substantial throughput gain as shown in Fig. 3. of [24].

For SM, the BER calculations is carried out using equations 19 and the technique for selecting the best BER is the same as in RC. The verall throughput is calculated using the following equation:

$$
T_{h(\text { MIMO) }}(x, y, z)=T h_{\mathrm{BER}_{M i}}
$$

For ASM, after the process explained in subsection (c) of (III) has been performed, the BER calculations is carried out based on equation (22), and also choose the highest modulation level which gives the highest throughput such that the BER is less than $10^{-2}$. The overall throughput is calculated using the following equation:

$$
T_{h(\mathrm{MIMO})}(x, y, z)=T h_{\mathrm{BER}_{N_{T M}}}
$$

Where, $N_{T}$ denotes number of selected transmitters (LEDs) in a given channel rank condition.

For SMP we use the similar approach as in RC except that, in this case we use Adaptive modulation with PARC [25] to optimize the choice of modulation separately for each transmitter. For each possible set of modulation schemes, we compute the BER expression as in equation (25) and choose the highest modulation level which gives the highest throughput such that the BER is less than $10^{-2}$ : 


$$
T_{h(\mathrm{MIMO})}(x, y, z)=\sum_{i=1}^{N_{T}} T h_{\mathrm{BER}_{\mathrm{TX}{ }_{M M i}}}
$$

Where $T h_{\mathrm{BER}_{\mathrm{TX}{ }_{M} M_{i}}}$ denotes throughput for an individual LED with an appropriate modulation while $N_{T}$ presents the number of transmitting LEDs.

\section{Simulation PARAMETERS AND RESUlts}

We consider a $4 \times 4$ indoor MIMO scenario as in [24], but in this paper we also consider the effect of wall reflections. The system is located within a room of size $4 \times 4 \times 3 \mathrm{~m}$ and we assume the transmitters are placed at a height of $2.50 \mathrm{~m}$ and oriented downwards perpendicular to the floor of the room. The receiver is allowed to move randomly at a height of $0.75 \mathrm{~m}$ (human waist or table height) and its detectors are either placed vertically or oriented at a given elevation angle as in Fig. 1 (d). The inclined detector's orientation is meant to increase the likelihood of FOV overlap between the transmitters and the receivers and also incoming reflected rays to the receiver, thus increasing system spectral efficiency.

A computer program that implements the scenarios presented in previous sections was written using MATLAB software. The simulation parameters are tabulated in Table I. The room size shows the dimension of the room where the simulation is assumed. The TX/RX setup defines the number of transmitters and receivers used in simulation. The TX separation shows how far the four transmitting LEDs are separated from each other, the RX separation shows how the receiver detectors are separated (this applies for the vertically oriented receiver). The TX/RX FOV are the Field of View angles of the transmitter and receiver respectively. The RX detectors elevation angle is the one between detector's axis and the horizontal. For angular oriented detectors (A. detectors) this angle is varied to determine the optimum one while for the vertically oriented detectors (V. detectors) the angle of elevation is always $90^{\circ}$ from the horizontal plane. The RX detectors azimuth angle separation shows how four detectors are angularly separated around $360^{\circ}$ for the angular diversity detectors setup (A. detectors). We assume users hold their devices in a random orientation in the range of $0^{\circ}-360^{\circ}$ azimuth angles from the position of the detector. This is achieved by applying (12) to all the detector locations. The experiment set-up and system performance comparison are presented in the next subsections: 
TABLE I. PARAMETERS USED FOR SIMULATION

\begin{tabular}{|c||c|}
\hline \multicolumn{1}{|c||}{ Parameters } & Values \\
\hline Room size $(W \times L \times H)$ & $4 \mathrm{~m} \times 4 \mathrm{~m} \times 3 \mathrm{~m}$ \\
\hline Number of TX/RX & $4 \times 4$ \\
\hline TX separation & $0.6 \mathrm{~m}$ \\
\hline Reflection coefficients $(\alpha)$ & $0.1,0.3,0.5,0.7,0.9$ \\
\hline Reflection parameter $\left(m_{s}\right)$ & 1 (rough surface) \\
\hline RX separations (V. detectors) & $0.1 \mathrm{~m}$ \\
\hline Photodiode responsivity $(\varrho)$ & $1 \Lambda / \mathrm{W}$ \\
\hline RX random rotation angles & $0^{\circ}$ to $360^{\circ}$ \\
\hline TX/RX FOV & $45^{\circ}, 45^{\circ}$ \\
\hline RX elevation angle (A. Diversity) & $45^{\circ}$ to $90^{\circ}$ towards vertical \\
\hline RX azimuth angle separation (A. Diversity) & $90^{\circ}$ \\
\hline Photodiode area $(A)$ & $1 \mathrm{~cm}{ }^{2}$ \\
\hline MIMO transmission techniques & $\mathrm{RC}, \mathrm{SM}, \mathrm{ASM}, \mathrm{SMP}$ \\
\hline Modulation schemes & $2 \mathrm{PAM}-1024 \mathrm{PAM}$ \\
\hline
\end{tabular}

\section{A. Setting up Vertical detectors}

To validate our results, the setup developed in [18] was repeated for all proposed MIMO transmission techniques and different TX separations $(0.2 \mathrm{~m}, 0.4 \mathrm{~m}, 0.6 \mathrm{~m})$. The RX detector separation remains $0.1 \mathrm{~m}$ all the time with a vertically oriented $\mathrm{RX}$ as in Fig. 1(d) right. The BER results for a data rate of $\mathrm{R}=4 \mathrm{bps} / \mathrm{Hz}$ are plotted as shown in Fig. 2 of [24]. These results match well with Fig. 3(a) of [18] and show the validity of the simulator.

We then developed a mobile receiver model and evaluated the throughput for 1000 locations. In this case (of a mobile receiver) we employ adaptive modulation for all transmission techniques, i.e. the rate of transmission is updated at each room location depending on the channel conditions. In addition to adaptive modulation we consider PARC in SMP. The results for average throughputs of the different transmission techniques and different reflection coefficients are compared in Table III and IV. Further, the CDF for all techniques are shown in Fig. 5, 6, 7 and 8. Both data tables and CDF plots are explained in subsection V-B.

B. Comparison between Vertical detectors and Angular diversity detectors scenarios with specular and diffuse reflections.

Here we compare the performance of vertical detectors and angular diversity detectors for a mobile receiver taking into consideration the effects of wall reflections and receiver. All the setups were simulated using all the MIMO transmission techniques discussed in section III. Using the throughput calculations in section IV, typical average throughput results for all setups 
(vertically oriented and angular diversity detectors) and their scenarios (reflection types and reflection coefficients) are tabulated in Table III and IV. Results are shown for four techniques (RC, SM, ASM and SMP), with both V. detectors (Vertically oriented detectors) and A. detectors (angular diversity detectors). Fig. 4. shows the SMP CDF comparison for three different elevation angles in angular diversity receiver $\left(10^{\circ}, 15^{\circ}\right.$ and $\left.20^{\circ}\right)$ when LOS, LOS + Df and LOS + Sp are considered. Elevation angles. Looking at the A. detectors (LOS + Dif) results on the same figure we can see that elevation angles of $10^{\circ}, 15^{\circ}$ and $20^{\circ}$ give throughputs of $24.8 \mathrm{bps} / \mathrm{Hz}, 29.2 \mathrm{bps} / \mathrm{Hz}$ and $22.3 \mathrm{bps} / \mathrm{Hz}$ respectively and therefore $15^{\circ}$ is used in all the subsequent simulation results. Fig. 5. shows the RC CDF comparison between V. detectors and A. detectors with and without reflections, also with different reflection coefficients. For simplicity only the CDF results for reflection coefficients of $0.3,0.5$, and 0.7 are plotted for all the curves, other results are recorded in Tables III and IV.

1) Results for the LOS channel:

Table II shows results for different antenna separations matching to [24] where the four techniques (RC, SM, ASM, SMP) in LOS were compared in detail. Looking at the table we can see that, for a moving angular diversity receiver there is an improvement in throughput when using SMP with PARC compared to other techniques (RC, SM and ASM). Percentage wise, for a TX separation of $0.4 \mathrm{~m}$, SMP performs $46 \%$ and $144 \%$ better than RC for V. Detectors and A. Detectors respectively. In comparison to ASM, SMP performs $41 \%$ and $120 \%$ better for V. Detectors and A. Detectors respectively. For a TX separation of $0.6 \mathrm{~m}$, SMP performs $120 \%$ and $192 \%$ better than RC for V. Detectors and A. Detectors respectively and when compared to ASM, SMP performs $78.9 \%$ and $85.1 \%$ better for V. Detectors and A. Detectors respectively. All the subsequent results in this paper are for $0.6 \mathrm{~m}$ transmitter spacing as this spacing provides the best performance in Table II. Here, we also consider the channel rank of different receiver locations in the room. Looking at Fig. 3(a) we can see that the channel matrix rank for the vertical detector is 1 for a large area of the room (60\%) while in Fig. 3(b) the proportion is reduced for the angular diversity detectors (44\%) which indicates a higher potential for MIMO receivers. We can see in Fig. 5. that the difference between the two setups in LOS conditions mainly relates to the coverage where $10 \%$ of the V. detectors setup give zero throughput to the total loss of LOS paths between TX and RX. The A. detectors setup gives non zero throughput 
results in all room locations.

2) Results for the LOS with specular reflection channel:

We can also see the effects of reflections when we look at Fig. 3(c) through (h). In Fig. 3(c) when specular reflection with a reflection coefficient $\alpha=0.3$ is considered in $\mathrm{V}$. detectors, the proportion of rank 1 channel reduces to $27 \%$ and rank 2 increases to $55 \%$. In Fig. 3(d) when specular reflections $(\alpha=0.3)$ are included in the A. detectors setup, the channel matrix rank values are either only $3(30 \%)$ and $4(70 \%)$ as the chance of multipath reception has increased. Also in Fig. 3(e) when specular reflections $(\alpha=0.5)$ are included in the V. detectors setup, the channel matrix rank is either $3(33 \%)$ or $4(68 \%)$ as the chance of multipath reception has further increased compared to $\mathrm{V}$. detectors $(\alpha=0.3)$. Fig 3(f) shows the effect of specular reflections on A. detectors $(\alpha=0.5)$, the rank 4 case dominates $(90 \%)$ and only $10 \%$ of channels have rank 3 .

Looking at Table III, the $\mathrm{RC}$ results show that the average throughput for all specular reflection coefficients does not vary significantly because the same data are transmitted by all transmitting LEDs so whether the rank of channel matrix is 1 or 4 , the same data rate is achieved. The specular reflection results in Fig. 5 shows that both detectors setups give non zero throughput because of the gain caused by the specular reflected rays. Also both detector setups have negligible difference in their throughput perfromance because RC cannot exploit higher MIMO channel rank to increase throughput.

Table III shows the average throughput for SM with specular reflections. It can be seen that SM shows modest improvement even as the specular reflection coefficient $(\alpha)$ increases. It can be seen that the A. detectors setup shows much better (almost 100\%) performance improvement compared to the V. detectors for the same reflection coefficient $(\alpha=0.5)$. Fig. 6. shows CDF results for SM where we can see that both A. Detector and V. detectors have poor performance due to rank deficient conditions in many locations of the room. This problem is addressed by the ASM approach proposed in this paper.

In Table III the average throughput for ASM improves as the specular reflection coefficient $\alpha$ increases for both two receiver setups. It is shown that the A. detectors has $17 \%$ throughput improvement compared to $\mathrm{V}$. detectors for the same reflection coefficient $(\alpha=0.5)$. Fig. 7 . shows the CDF results for ASM with three reflection coefficients where we can see that the 
TABLE II. SIMULATION RESULTS FOR LOS WITH DIFFRENT ANTENNA SEPARATIONS

\begin{tabular}{|c||c|c|c|c|c|c|}
\hline \multicolumn{1}{|c||}{\multirow{2}{*}{$\begin{array}{c}\text { Trans- } \\
\text { mission } \\
\text { Method }\end{array}$}} & \multicolumn{5}{c|}{ Average throughput in bps/Hz } \\
\cline { 2 - 7 } & $\mathbf{0 . 2} \mathbf{~ m}$ & $\mathbf{0 . 4} \mathbf{~ m}$ & $\mathbf{0 . 6} \mathbf{~ m}$ & $\mathbf{0 . 2} \mathbf{~ m}$ & $\mathbf{0 . 4} \mathbf{~ m}$ & $\mathbf{0 . 6} \mathbf{~ m}$ \\
\hline RC & 5.2 & 5.6 & 6 & 6.5 & 6.3 & 6.4 \\
\hline SM & 1.1 & 1.3 & 1.8 & 1.7 & 2.2 & 2.9 \\
\hline ASM & 5.2 & 5.8 & 7.6 & 6.1 & 7 & 10.1 \\
\hline SMP & 6 & 8.2 & 13.2 & 13 & 15.4 & 18.7 \\
\hline
\end{tabular}

Key to the table:

$\mathrm{V}=$ Vertically oriented, $\mathrm{A}=$ Angular diversity

$0.2 \mathrm{~m}$ to $0.6 \mathrm{~m}$ are the transmitter separations

A. Detector achieves better coverage and hence throughput improvement. The figure shows that both setups achieve non zero throughput in $100 \%$ of the room locations for this propagation environment. Comparing the results of LOS and the effect of specular reflection at $\alpha=0.5$ we can see that both setups have $100 \%$ coverage but there is $22 \%$ throughput improvement for A. detectors over V. detectors. Looking at SMP, it shows a significant throughput improvement compared to RC, SM and ASM. This is particularly true because the channel rank for angular diversity detectors is increased to a higher likelihood of LOS propagation between TX and RX compared to vertically oriented detectors and hence a gain in throughput is observed. Taking reflection coefficient $\alpha=0.5$ we can see that SMP performs $100 \%$ and $175 \%$ better than RC for V. Detectors and A. Detectors respectively and when compared to ASM, SMP performs 59\% and $89 \%$ better for V. Detectors and A. Detectors respectively. We can also notice that increasing $\alpha$ causes a significant improvement in throughput. Fig. 8. also shows that when SMP is used there is a significant difference in throughput between the two setups but that coverage of $100 \%$ is achieved for both cases. In the case of specular reflections, the A. detectors shows a $40 \%$ improvement compared to the V. Detector. 


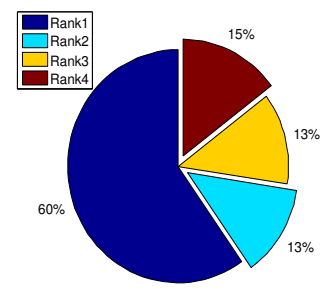

(a) V. detectors LOS

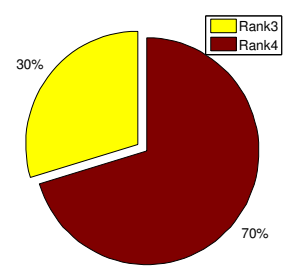

(d) A. detectors $(\alpha=0.3)$

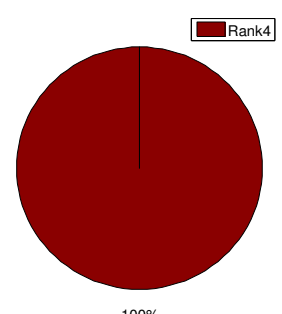

(g) V. detectors

( $\alpha=$ either 0.3 or 0.5 )

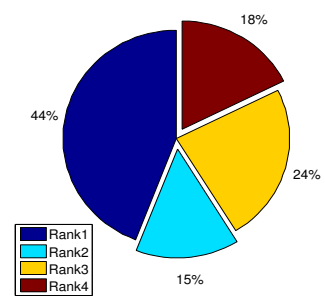

(b) A. detectors (LOS)

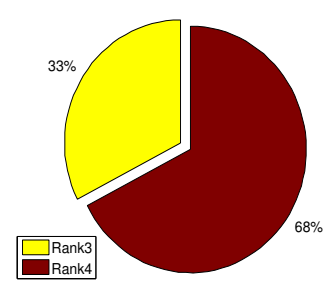

(e) V. detectors $(\alpha=0.5)$

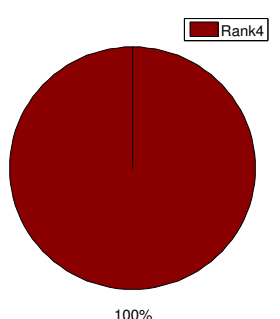

(h) A. detectors

( $\alpha=$ either 0.3 or 0.5 )

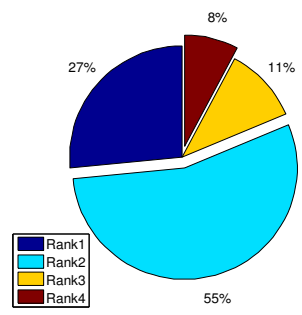

(c) V. detectors $(\alpha=0.3)$

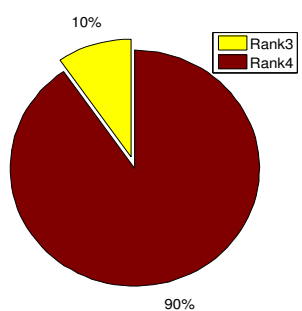

(f) A. detectors $(\alpha=0.5)$

Fig. 3. Percentage distributions of MIMO channels matrix rank in 1000 locations of the room (a) Vertical detectors setup (b) Angular diversity detectors setup (c \& e) Vertical detector setup with specular reflections (d \& f) Angular diversity detectors setup with specular reflections (g \& h) Both vertical and angular detectors setups with diffuse reflections 


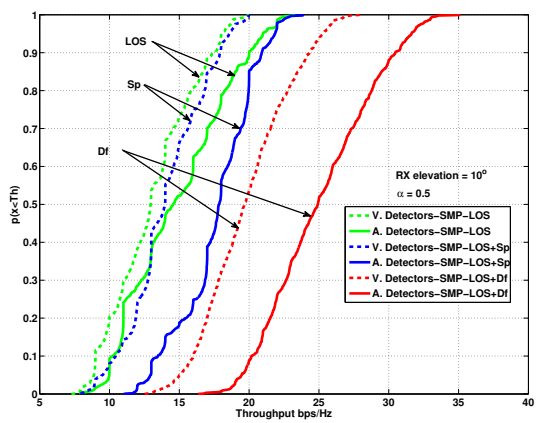

(a)

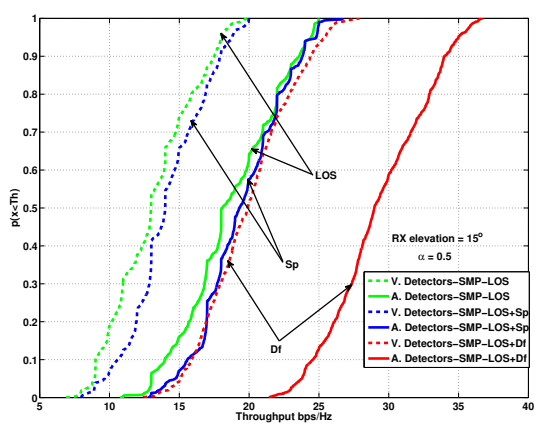

(b)

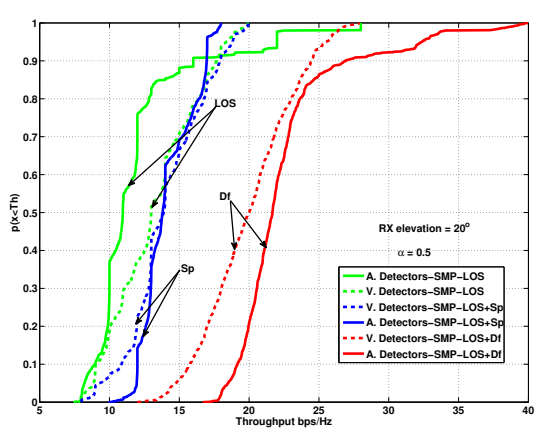

(c)

Fig. 4. SMP CDF comparison between three elevation angles in angular diversity receiver detectors when LOS, LOS + Df and LOS + Sp are considered, $\alpha=0.5$ (a) SMP, $10^{\circ}$ (b) SMP, $15^{\circ}$ (c) SMP, $20^{\circ}$ 


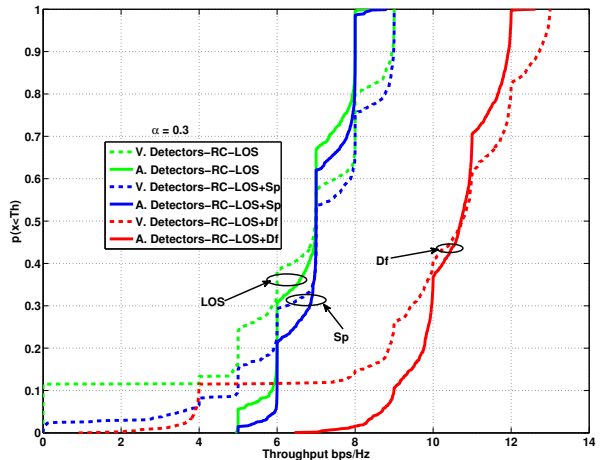

(a)

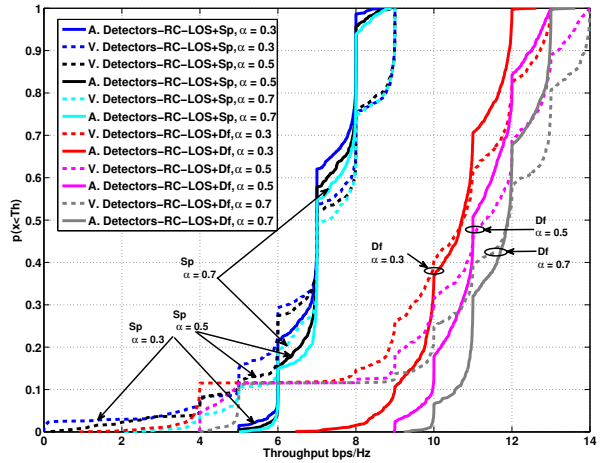

(b)

Fig. 5. RC CDF comparison between three reflection coefficients in vertically and angular diversity receiver detectors when LOS, LOS $+\mathrm{Sp}$ and LOS + Df are considered (a) LOS Vs LOS + Sp, $\alpha=0.3$ (b) LOS + Sp Vs LOS + Df, $\alpha=0.3,0.5$ and 0.7.

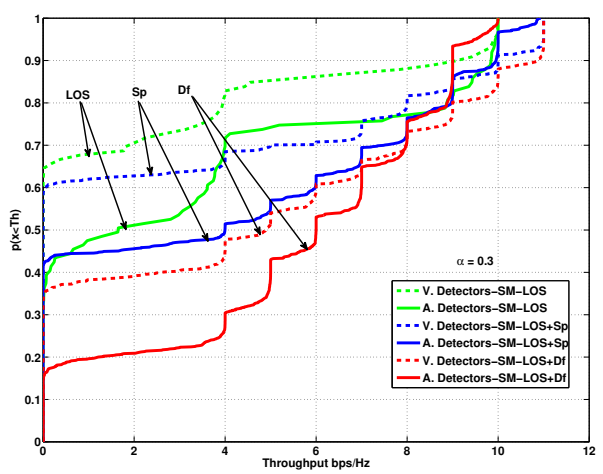

(a)

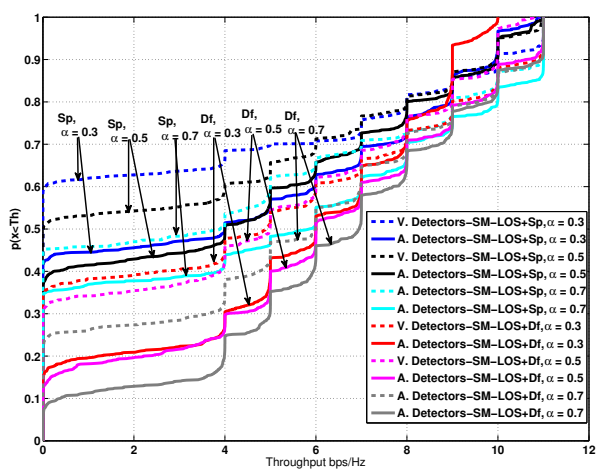

(b)

Fig. 6. SM CDF comparison between three reflection coefficients in vertically and angular diversity receiver detectors when LOS, LOS $+\mathrm{Sp}$ and LOS + Df are considered (a) LOS Vs LOS + Sp, $\alpha=0.3$ (b) LOS + Sp Vs LOS + Df, $\alpha=0.3,0.5$ and 0.7. 


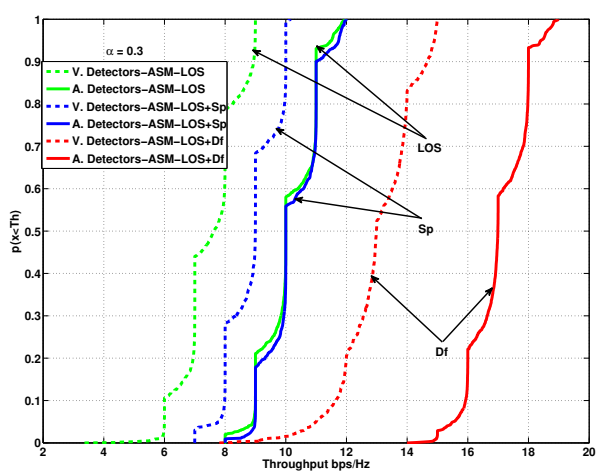

(a)

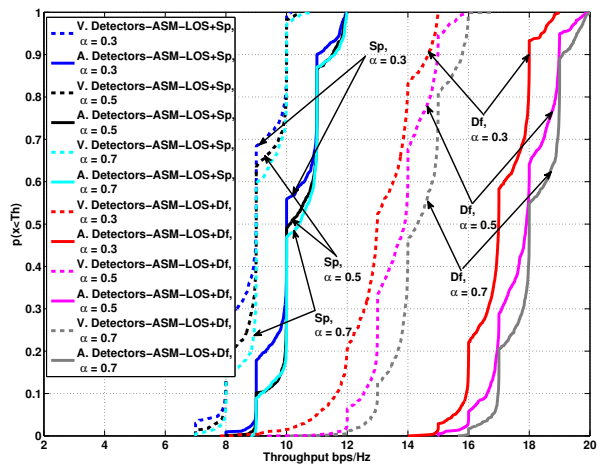

(b)

Fig. 7. ASM CDF comparison between three reflection coefficients in vertically and angular diversity receiver detectors when LOS, LOS + Sp and LOS + Df are considered (a) LOS Vs LOS + Sp, $\alpha=0.3$ (b) LOS + Sp Vs LOS + Df, $\alpha=0.3,0.5$ and 0.7.

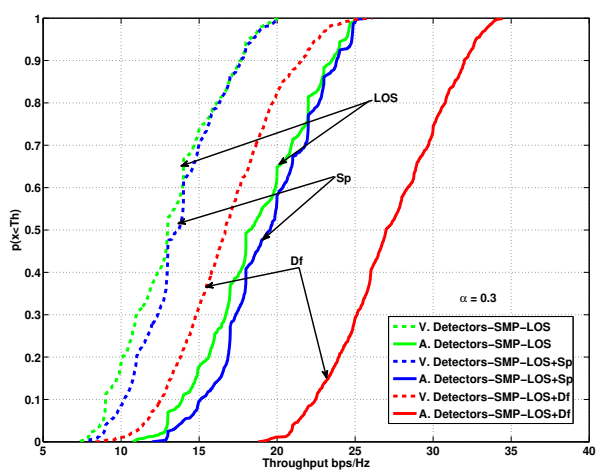

(a)

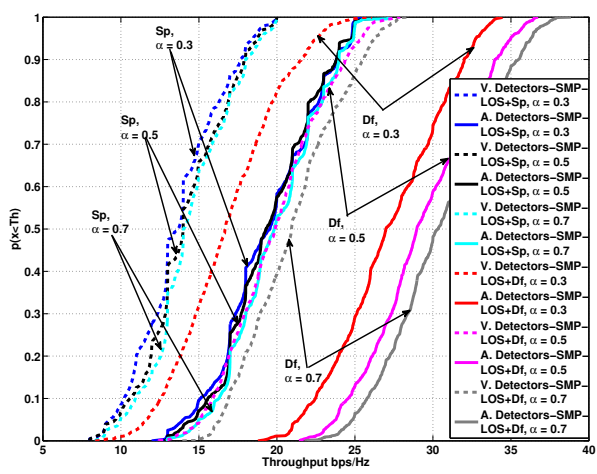

(b)

Fig. 8. SMP CDF comparison between three reflection coefficients in vertically and angular diversity receiver detectors when LOS, LOS + Sp and LOS + Df are considered (a) LOS Vs LOS + Sp, $\alpha=0.3$ (b) LOS + Sp Vs LOS + Df comparison, $\alpha=0.3,0.5$ and 0.7. 
TABLE III. SimUlation RESULtS FOR LOS WITH SPECULAR REFLECTIONS

\begin{tabular}{|c||c|c|c|c|c|c|c|c|c|c|c|c|}
\hline \multicolumn{1}{|c||}{$\begin{array}{c}\text { Trans- } \\
\text { mission } \\
\text { Method }\end{array}$} & \multicolumn{10}{|c|}{ Average throughput in bps/Hz } \\
\cline { 2 - 14 } & \multicolumn{10}{|c|}{ V. detectors $\left(0^{\circ}\right)$} & A. detectors $\left(15^{\circ}\right)$ \\
\hline RC & 6.0 & 0.1 & $\mathbf{0 . 3}$ & $\mathbf{0 . 5}$ & $\mathbf{0 . 7}$ & $\mathbf{0 . 9}$ & LOS & $\mathbf{0 . 1}$ & $\mathbf{0 . 3}$ & $\mathbf{0 . 5}$ & $\mathbf{0 . 7}$ & $\mathbf{0 . 9}$ \\
\hline SM & 1.8 & 1.8 & 1.9 & 7.0 & 7.1 & 7.3 & 6.4 & 6.9 & 7.0 & 7.1 & 7.2 & 7.3 \\
\hline ASM & 7.5 & 7.5 & 8.6 & 8.8 & 9.8 & 4.1 & 2.9 & 3.1 & 3.9 & 4.2 & 6.3 & 7.0 \\
\hline SMP & 13.1 & 13.3 & 13.7 & 14.0 & 14.3 & 14.6 & 18.6 & 18.8 & 19.1 & 19.5 & 20.0 & 20.4 \\
\hline
\end{tabular}

Key to the table:

$\mathrm{V}=$ Vertically oriented, $\mathrm{A}=$ Angular diversity

Parameters 0.1 to 0.9 are the reflection coefficients $(\alpha)$ of the reflecting surface

3) Results for LOS with diffuse reflection channel:

Table IV shows simulation results when diffuse reflections are considered. The diffuse reflection case shows a significant effect on system performance. Considering the $\alpha=0.5 \mathrm{RC}$ results for the diffuse reflection model shows a $47-52 \%$ improvement compared to those for specular reflection (Table III) in both A. detectors and V. detectors. Fig. $3 \mathrm{~g}$ and $\mathrm{h}$ show that when diffuse reflections are considered in both $\mathrm{V}$. detectors and A. detectors, the channel matrix rank is always $4(100 \%)$ for all the reflection coefficients $(\alpha=0.1$ through 0.9$)$. This is because the diffuse reflection scattered optical channel makes it possible to obtain full MIMO performance gains. Looking at the ASM performance in the model including diffuse reflections, we see also a substantial improvement in throughput. Table IV shows that ASM $(\alpha=0.5)$ has $41-56 \%$ throughput improvement over the specular reflection results in Table III. For SMP, when diffuse reflections are considered it also shows significant improvement. At $\alpha=0.3$ in A. detectors, SMP has an improvement of $20-37 \%$ over the scenario when only specular reflection is included. In terms of the comparison between $\mathrm{V}$. detector and A. detectors in diffuse reflection conditions with $\alpha=0.5$, ASM with A. detectors shows $28 \%$ throughput improvement over ASM with V. detectors while SMP with A. detectors shows $48 \%$ throughput improvement over SMP with V. detectors. Again we can see that, SMP performs $89 \%$ and $170 \%$ better than RC for V. Detectors and A. Detectors respectively. Generally the diffuse reflection model has shown a big impact on indoor OWC performance due to the of scattered optical power improving the MIMO channel rank statistics. 
TABLE IV. SIMULATION RESULTS FOR LOS WITH DIFFUSE REFLECTIONS

\begin{tabular}{|c|c|c|c|c|c|c|c|c|c|c|c|c|}
\hline \multirow{3}{*}{$\begin{array}{l}\text { Trans- } \\
\text { mission } \\
\text { Method }\end{array}$} & \multicolumn{12}{|c|}{ Average throughput in bps $/ \mathrm{Hz}$} \\
\hline & \multicolumn{6}{|c|}{ V. detectors $\left(0^{\circ}\right)$} & \multicolumn{6}{|c|}{ A. detectors $\left(15^{\circ}\right)$} \\
\hline & LOS & 0.1 & 0.3 & 0.5 & 0.7 & 0.9 & LOS & 0.1 & 0.3 & 0.5 & 0.7 & 0.9 \\
\hline $\mathbf{R C}$ & 6.0 & 6.2 & 9.6 & 10.4 & 10.9 & 11.1 & 6.4 & 6.8 & 10.3 & 10.8 & 11.3 & 11.5 \\
\hline SM & 1.8 & 3.5 & 4.8 & 5.1 & 6.0 & 6.8 & 2.9 & 4.9 & 6.1 & 6.4 & 6.9 & 7.6 \\
\hline ASM & 7.5 & 7.5 & 13.2 & 13.7 & 14.3 & 14.6 & 10.0 & 14.6 & 16.9 & 17.6 & 18.0 & 18.4 \\
\hline SMP & 13.1 & 13.1 & 16.5 & 19.7 & 20.8 & 21.6 & 18.6 & 22.4 & 26.2 & 29.2 & 30.4 & 31.6 \\
\hline
\end{tabular}

Key to the table:

$\mathrm{V}=$ Vertically oriented, $\mathrm{A}=$ Angular diversity

Parameters 0.1 to 0.9 are the reflection coefficients $(\alpha)$ of the reflecting surface

\section{CONCLUSION}

We have presented a mobile angular diversity optical receiver detector model with both specular and diffuse reflections in an indoor visible light communications system. We applied two different reflection models and used a range of reflection coefficients to model different types of reflecting surfaces. We applied different MIMO transmission techniques with adaptive modulation and Per Antenna Rate Control to evaluate the throughput across different room locations. We have compared results for vertical detectors and angular diversity detectors setups in different scenarios. Our initial results show that using angular diversity detectors increase the likelihood of LOS among transmitters and receivers. It is also shown that diffuse reflections have a significant positive impact in system throughput compared to the specular case and both are better than for LOS propagation.

It is shown that for same setup SMP has better performance compared to other candidates (RC, and ASM). Percentage wise, for specular reflections SMP performs $100 \%$ and $175 \%$ better than $\mathrm{RC}$ for V. detectors and A. detectors respectively while in diffuse reflection SMP performs $89 \%$ and $170 \%$ better than $\mathrm{RC}$ for $\mathrm{V}$. detectors and A. detectors respectively. Compared to ASM, in specular reflections SMP performs $60 \%$ and $90 \%$ better for V. detectors and A. detectors respectively while in in specular reflections SMP performs $43 \%$ and $66 \%$ better for V. detectors and A. detectors respectively. It was also seen that, using ASM where we switch between different TX/RX setups, transmission techniques and modulation, we could achieve throughput improvements compared to always using SM with 4 transmitters.

Looking at both LOS, LOS + Sp, and LOS + Df results we can see that ASM performs much better than SM due to its robustness to rank deficient channels. In LOS, ASM performs 317\% 
better than SM with V. detectors and $245 \%$ better than SM with A. detectors. In the specular reflection case when we consider $\alpha=0.5$, ASM performs 300\% better than SM with V. detectors and $145 \%$ better than SM in A. detectors. Also in the diffuse refection case when considering the same reflection coefficient, ASM performs $168 \%$ better than SM in V. detector and $175 \%$ better than SM in A. detectors. Generally our simulations suggest that, for mobile optical receivers angular diversity detectors can perform better than vertical oriented receivers. When specular or diffuse reflections are included the system performance improves significantly. We have seen the positive impact of reflected optical paths on conditions for the scenarios discussed in this paper. This is because, in the diffuse channels model, receivers can exploit both LOS paths and reflected paths.

REFERENCES

[1] R. Mesleh and H. Haas, "Indoor Optical Wireless Communications; Potential and state-ofart” IEEE Communication magazine, Vol. 49, pp. 56-62, September. 2011.

[2] L. Zeng et al. "High data rate multiple input multiple output (MIMO) optical wireless communications using white led lighting”, IEEE Journal in selected Areas in Communications, Vol 27, pp 1654-1662, December. 2009.

[3] Y. Tanaka et al. "Indoor Visible Light Data Transmission system utilizing White LED lights", IEICE Transaction on communications, Vol 8. pp. 2440-2454. Aug. 2003.

[4] D. De-qian and K. Xi-zheng, "A new indoor VLC channel model based on reflection" Springer optoelectronics letters, Vol 6, no 4. pp. 295-298, July. 2010.

[5] J. Armstrong, B. Schmidt, "Comparison of asymmetrically clipped optical OFDM and DCbiased Optical OFDM in AWGN", IEEE Communication letters, Vol. 12, pp. 343-345, May. 2008.

[6] J. Armstrong, "OFDM for Optical Communications" IEE Journal of light wave technology, Vol. 27, no. 3, February. 2009.

[7] P. Fahamuel, J. Thompson and H. Haas, "Study, analysis and application of optical OFDM, single carrier (SC) and MIMO in intensity modulation direct detection (IM/DD)" IET Intelligent Signal Processing Conference 2013, London, pp. 1-6, December. 2013. 
[8] K. Dambul, D. O’Brien, and G. Faulkner, "Indoor Optical Wireless MIMO System With an Imaging Receiver" IEEE Photonics Technology Letters, Vol. 23, no.2, pp. 97-99, January. 2011.

[9] D. O’Brien, "Multi-input multi-output (MIMO) indoor optical wireless communications" Conference Record of the Forty-Third Asilomar Conference on Signals, Systems and Computers, Pacific Grove, CA, pp. 1636-1639, November. 2009.

[10] L. Tang and W. Chang, "Design of an Omnidirectional Multibeam Transmitter for HighSpeed Indoor Wireless Communications" EURASIP Journal on Wireless Communications and Networking 2010, Vol 2010, no 65, pp. 1687-1499, April. 2010.

[11] J. Kahn and J. Barry, "Wireless Infrared Communications" IEEE Proceedings, Vol 85. no 2, pp. 265 - 298, August. 2012.

[12] C. Pei et al. "Design of Indoor Optical Wireless Collaborative Cellular system", IEEE Wireless Communications and Signal Processing, Nanjin, Vol. 1, pp.1-6, November. 2011.

[13] R. Mesleh et al, "Indoor MIMO Optical Wireless Communication Using Spatial Modulation” IEEE International Conference on Communications (ICC), Cape Town, South Africa. pp. 1-5, May. 2010.

[14] T. Fath, H. Haas, M. Renzo and R. Mesleh "Spatial Modulation applied to Optical Wireless Communications in Indoor LOS Environments" IEEE Globe Telecommunications conference (GLOBECOM 2011), Houston, TX, USA, pp. 1-5, December. 2011.

[15] T. Fath, J. Klaue and H. Haas "Coded Spatial Modulation applied to Optical Wireless Communications in Indoor Environments" IEEE Wireless Communications and Networking Conference, Shanghai, China, pp. 1000-1004, April. 2012.

[16] T. Wang, Y. Sekercioglu and J. Armstrong, "Hemispherical Lens Based Imaging Receiver for MIMO Optical Wireless Communications" IEEE Workshop on Optical Wireless Communications, Anaheim, CA, pp.1239-1243, December. 2012.

[17] T. Wang, Y. Sekercioglu, and J. Armstrong, "Analysis of an Optical Wireless Receiver Using a Hemispherical Lens With Application in MIMO Visible Light Communications" Journal of Light Wave Technology, Vol 31, no 11. pp.1744-1754, June. 2013.

[18] T. Fath and H. Haas, "Performance Comparison of MIMO Techniques for Optical Wireless 
Communications in Indoor Environments" IEEE Transactions on Communications, Vol 61, no 2. pp. 733-742, February. 2013.

[19] D. Tsonev, S. Sinanovic and H. Haas, "Practical MIMO Capacity for Indoor Optical Wireless Communication with White LEDs" IEEE Vehicular Technology Conference (VTC Spring), Dresden, pp. 1-5, June. 2013.

[20] N. Saha, R. Mondal and Y. Jang, "Receiver Performance Improvement Utilizing Diversity in MIMO VLC" International Conference on ICT Convergence (ICTC), Jeju. pp. 546-549, October. 2013.

[21] T. Wang and J. Armstrong, "Performance of Indoor MIMO Optical Wireless System Using Linear Receiver with Prism Array" 2014 Australian Communications Theory Workshop (AusCTW), Sydney, NSW, pp. 51-56, February. 2014.

[22] T. Wang, R. Green, and J. Armstrong, "Prism Array-Based Receiver with Application in MIMO Indoor Optical Wireless Communications" International Conference on Transparent Optical Networks (ICTON), Graz, Australia, pp. 1-4, July. 2014.

[23] Y. Wang and N. Chi, "Demonstration of High-Speed $2 \times 2$ Non-Imaging MIMO Nyquist Single Carrier Visible Light Communication With Frequency Domain Equalization” Journal of Light Wave Technology, Vol. 32, no. 11, pp. 2087-2093, June. 2014.

[24] P. Fahamuel, J. Thompson and H. Haas, "Improved Indoor VLC MIMO channel capacity using mobile receiver with angular diversity detectors" IEEE Globe Telecommunications conference (GLOBECOM 2014), Austin, TX, USA, December. 2014.

[25] S. Grant et al, "Per Antenna-Rate-Control (PARC) in Frequency selective Fading with SICGRAKE Receiver" IEEE Vehicular Technology Conference, Vol 7, pp. 26-29, September. 2004.

[26] R. Mesleh et al, "Optical Spatial Modulation" Journal of Optical Communications and Networking, IEEE/OSA, vol 3, no 3, pp. 234-244, March 2011.

[27] L. Zeng et al, "Improvement of Data Rate by using Equalization in an Indoor Visible Light Communication System", IEEE International Conference on Circuits and Systems for Communications, 2008. ICCSC 2008, Shanghai,pp. 678-682, May, 2008.

[28] R. Masleh et al. "Spatial Modulation-A New Low Complexity Spectral Efficiency Enhanc- 


\section{References}

[1] R. Mesleh and H. Haas, "Indoor Optical Wireless Communications; Potential and Stateof- the Art," in IEEE Communication Magazine, vol. 49, pp. 56-62, September 2011.

[2] Roke Manor Research Ltd, "UK-Spectrum allocations." http://www.roke.co. uk/resources/datasheets/UK-Frequency-Allocations.pdf. Chemring technology solutions, UK Radio Frequency Allocations Chart, Accessed April 29, 2015.

[3] Cisco, "Global Mobile Data Traffic Forecast Update, 2013-2018." http://www . cisco.com/c/en/us/solutions/collateral/service-provider/ visual-networking-index-vni/white_paper_c11-520862.html. Accessed August 18, 2014.

[4] G. Research, "Visible Light Communication (VLC) - A Potential Solution to the Global Wireless Spectrum Shortage." http://www.gbiresearch.com/. Accessed August 18, 2014.

[5] SPIE, "High-Speed Wireless Networking Using Visible Light." http: / / spie.org/ x93593.xml을 2015/08/2014. Accessed August 18, 2014.

[6] T. Fath, Evaluation of Spectrally Efficient Indoor Optical Wireless Transmission Techniques. PhD thesis, University of Edinburgh, October 2013.

[7] IEEE Std. 802.15.7-2011, "IEEE Standard for Local and Metropolitan Area Networks, Part 15.7: Short-Range Wireless Optical Communication Using Visible Light.” IEEE explorer. IEEE Std, 2011.

[8] L. Zeng and D. O'Brien, "High data rate Multiple Input Multiple Output MIMO Optical Wireless Communications using white LED lighting," in IEEE Journal on Selected Areas in Communications, vol. 27, pp. 1654-1662, December 2009.

[9] J. Armstrong and B. Schmidt, "Comparison of Asymmetrically Clipped Optical OFDM and DC-biased Optical OFDM in AWGN," in IEEE Communication letters, vol. 12, pp. 343-345, May 2008.

[10] F. Gfeller and U. Bapst, "Wireless In-House Data Communication via Diffuse Infrared Radiation," in Proceedings of the IEEE, vol. 67, pp. 1474-1486, November 1979.

[11] G. Marsh and J. Kahn, "Performance Evaluation of Experimental 50-Mb/s Diffuse Infrared Wireless Link using on-off Keying with Decision-Feedback Equalization," in IEEE Transactions on Communications, vol. 44, pp. 1496-1504, November 1996.

[12] J. Carruthers and J. Kahn, "Angle Diversity for Nondirected Wireless Infrared Communication," in IEEE Transactions on Communications, vol. 48, pp. 960-969, June 2000. 
[13] Y. Tanaka, T. Komine, S. Haruyama, and M. Nakagawa, "Indoor Visible Light Data Transmission system utilizing white LED lights," in IEICE Transaction on communications, vol. 8, pp. 2440-2454, August 2003.

[14] J. Vucic, C. Kottke, S. Nerreter, K. Langer, and J. Walewski, "513 Mbit/s Visible Light Communications Link Based on DMT-Modulation of a White LED," in Journal of Lightwave Technology, vol. 28, pp. 3512-3518, December 2010.

[15] D. Tsonev, H. Chun, S. Rajbhandari, J. McKendry, S. Videv, E. Gu, M. Haji, S. Watson, A. Kelly, G. Faulkner, M. Dawson, H. Haas, and D. O'Brien, "A 3-Gb/s Single-LED OFDM-Based Wireless VLC Link Using a Gallium Nitride $\mu$ LED," in IEEE Photonics Technology Letters, vol. 26, pp. 637-640, April 2014.

[16] R. Mesleh, R. Mehmood, H. Elgala, and H. Haas, "Indoor MIMO Optical Wireless Communication Using Spatial Modulation," in IEEE International Conference on Communications (ICC), pp. 1-5, May 2010.

[17] C. Pei, D. Wu, Z. Zhang, and D. Zhang, "Design of Indoor Optical Wireless Collaborative Cellular System," in IEEE Wireless Communications and Signal Processing, pp. 1-5, November 2011.

[18] P. Fahamuel, J. Thompson, and H. Haas, "Study, Analysis and Application of Optical OFDM, Single Carrier (SC) and MIMO in Intensity Modulation Direct Detection (IM/DD)," in IET Intelligent Signal Processing Conference 2013, pp. 1-6, December 2013.

[19] P. Fahamuel, J. Thompson, and H. Haas, "Improved Indoor VLC MIMO Channel Capacity Using Mobile Receiver With Angular Diversity Detectors," in IEEE Globe Telecommunications conference (GLOBECOM 2014), December 2014.

[20] A. Still, Communications through the Ages: From sign Language to Television. New York: Murray Hill Books, 1946.

[21] R. Appleyard, Pioneers of of Electrical Communication. London: Macmillan, 1930.

[22] G. Vitetta, D. Taylor, G. Colavolpe, F. Pancaldi, and P. Martin, Wireless communications: algorithmic techniques. Chichester: John Wiley, 2013.

[23] N. Serafimovski, Extension and Practical Evaluation of the Spatial Modulation Concept. $\mathrm{PhD}$ thesis, University of Edinburgh, April 2013.

[24] A. G. Bell, Selenium and Photophone. Nature, 1880.

[25] S. Dimitrov, Analysis of OFDM-based Intensity Modulation Techniques for Optical Wireless Communications. PhD thesis, University of Edinburgh, August 2012.

[26] S. Hranilovic, Wireless Optical Communication Systems. McMaster University: Hamilton Ontario Canada, 2005.

[27] E. Hawks, Peoneer of Wireless. London: Methuen, 1927.

[28] C. Shannon, "A Mathematical Theory of Communication," in Bell System Technical Journal, pp. 623-656, July 1948. 
[29] C. Shannon, "Communication in the Presence of Noise," in Proc. of the IRE, pp. 10-21, January 1949.

[30] The Flag Press, "History Behind Semaphore Flags." http://flagexpressions. wordpress.com/2010/03/23/history-behind-semaphore-flags/.

The Flag Press: The real world on Flags, Accessed September. 12, 2014.

[31] A. Goldsmith, Wireless Communications. Cambridge University Press, 2005.

[32] T. Rappaport, Wireless Communications: Principle and Practice. 2nd edn Upper Saddle River NJ: Prentice Hall PTR, 2002.

[33] Uswitch, "History of mobile phones." http://www.uswitch.com/mobiles/ guides/history_of_mobile_phones. Accessed January 25, 2015.

[34] S. Sesia, I. Touk, and M. Baker, LTE-The UMTS Long Term Evolution:From Theory to Practice. 1st edn Eds: Wiley, 2009.

[35] W. Ng, T. Kanesan, Z. Ghassemlooy, and C. Lu, "Theoretical and Experimental Optimum System Design for LTE-RoF Over Varying Transmission Span and Identification of System Nonlinear Limit," in IEEE Photonics Journal, pp. 1560-1571, August 2012.

[36] A. Viterbi and R. Padovani, "Implications of Mobile Cellular CDMA," in IEEE Communications Magazine, pp. 38-41, December 1992.

[37] L. Brandenburg and A. Wyner, "Capacity of the gaussian channel with memory: The multivariate case," Tech. rep, Bell System, June 1974.

[38] D. Astely, E. Dahlman, A. Furuskar, Y. Jading, M. Lindstrom, and S. Parkvall, "LTE:The Evolution of Mobile Broadband," in IEEE Communications Magazine, pp. 44-51, December 2009.

[39] Motorola Inc, "Driving 4G: WiMAX and LTE." http://www.motorola.com, 20 07. Motorola Inc., positioning paper, Accessed Feb. 19, 2009.

[40] BROADCOM Corporation, "802.11n: Next-Generation Wireless LAN Technology." http: / / www.broadcom.com/docs/WLAN/802-11n-WP100-R.pdf. White paper BROADCOM Corporation Tech.report, Accessed Aug. 4, 2006.

[41] J. Kahn and J. Barry, "Wireless Infrared Communications," in Proceedings of the IEEE, vol. 85, pp. 265-298, February 1997.

[42] R. Weast, CRC Handbook of Chemistry and Physics. 64th edn CRC Press: Boca Raton, 1983.

[43] S. Dimitrov, R. Mesleh, H. Haas, M. Cappitelli, M. Olbert, and E. Bassow, "On the SIR of a Cellular Infrared Optical Wireless System for an Aircraft," in IEEE Journal on Selected Areas in Communications (IEEE JSAC), vol. 27, pp. 1623-1638, December 2009.

[44] W. Ng, W. Loedhammacakra, Z. Ghassemlooy, and R. Cryan, "Characterisation of a parallel optical all pass filter for chromatic dispersion equalisation in $10 \mathrm{~Gb} / \mathrm{s}$ system," in IET Circuits Devices and Systems, pp. 112-118, February 2008. 
[45] S. Alexander, Optical Communication Receiver Design. SPIE Press Book, 1997.

[46] D. O'Brien, G. Faulkner, S. Zikic, and N. Schmitt, "High Data-Rate Optical Wireless Communications in Passenger Aircraft: Measurements and Simulations," in 6th International Symposium on Communication Systems, Networks and Digital Signal Processing (CSNDSP08), vol. 27, pp. 68-71, July 2009.

[47] T. Fath and H. Haas, "Performance Comparison of MIMO Techniques for Optical Wireless Communications in Indoor Environments," in IEEE Transactions on Communications, pp. 733-742, February 2013.

[48] O. Bouchet, D. O’Brien, M. Tabach, M. Mach, G. Faulkner, L. Fernandez, M. Franke, J. Grubor, T. Kamalakis, Klaus.Langer, H. Mihn, I. Neokosmidis, S. Nerreter, G. Ntogari, J. Walewski, and M. Wolf, "State of the art-optical wireless," Tech. rep, OMEGA, January-November 2008.

[49] S. Haykin, Digital Communications. John Wiley and Sons: New York,NY, 1988.

[50] J. Barry, Wireless Infrared Communications. Kluwer Academic Publishers: Boston,MA, 1994.

[51] J. Carruthers and J. Kahn, "Modeling of nondirected wireless infrared channels," in IEEE Transactions on Communications, pp. 1260-1268, October 1997.

[52] International Electrotechnical Commission (IEC), "Safety of laser products part 1: Equipment classification, requirements and users guide," Group safety publication, reference number 825-1, IEC, November 1993.

[53] Osram Opto Semiconductors, "Datasheet: LCW W5SM Golden Dragon white LED." http://http://www.osram.de. Accessed April, 2011.

[54] S. Dimitrov, H. Haas, M. Cappitelli, , and M. Olbert, "On the Indoor Optical Wireless MIMO System With an Imaging Receiver Throughput of an OFDM-based Cellular Optical Wireless System for an Aircraft Cabin," in Proc. of European Conference on Antennas and Propagation (EuCAP 2011), pp. 11-15, April 2011. Invited paper.

[55] J. Proakis, Digital Communications 4th ed. McGraw-Hill, 2000.

[56] T. Elganimi, "Studying the BER Performance, Power and Bandwidth Efficiency for FSO Communication Systems under Various Modulation Schemes," in IEEE Jordan Conference on Applied Electrical Engineering and Computing Technologies (AEECT), pp. 1-6, December 2013.

[57] N. LaSorte, W. Barnes, and H.Refai, "The History of Orthogonal Frequency Division Multiplexing," in Global Telecommunications Conference, IEEE GLOBECOM 2008, pp. 1-5, November 2008.

[58] J. Armstrong and A. Lowery, "Power Efficient Optical OFDM," in Electronics Letters, pp. 370-372, March 2006.

[59] J. Armstrong, "OFDM for Optical Communications," in IEE Journal of light wave technology, vol. 27, pp. 189-204, February 2009. 
[60] D. Tse and P. Viswanath, Fundamentals of Wireless Communication. Cambridge University Press, 2005.

[61] R. Mesleh, H. Elgala, and H. Haas, "LED Nonlinearity Mitigation Techniques in Optical Wireless OFDM Communication Systems," in Journal of Optical Communications and Networking, IEEE/OSA, pp. 865-875, November 2012.

[62] G. J. Foschini, "Layered Space-Time Architecture for Wireless Communication in a Fading Environment when Using Multi-Element Antennas," in Bell Labs Technical Journal, pp. 41-59, October 1996.

[63] S. M. Alamouti, "A Simple Transmit Diversity Technique for Wireless Communications," in IEEE Journal on Selected Areas in Communications, pp. 1451-1458, October 1998.

[64] E. Telatar, "Capacity of Multi-Antenna Gaussian Channels," in European Transactions on Telecommunications, pp. 585-595, November 1999.

[65] V. Tarokh, N. Seshadri, and A. Calderbank, "Space-Time Codes for High Data Rate Wireless Communication: Performance Criterion and Code Construction," in IEEE Transactions on Information Theory, pp. 744-765, March 1998.

[66] G. Foschini and M. Gans, "On Limits of Wireless Communications in a Fading Environment when Using Multiple Antennas," in Wireless Personal Communications, pp. 311335, March 1998.

[67] P. Wolniansky, G. Foschini, G. Golden, and R. Valenzuela, "V-BLAST: an Architecture for Realizing very High Data Rates over the Rich-Scattering Wireless Channel," in Union Radio-Scientifique Internationale (URSI) Intern. Symp. on Signals, Systems, and Electronics (ISSSE), pp. 295-300, October 1998.

[68] H. Jafarkhani, Space-Time Coding: Theory and Practice. Cambridge University Press, 2005.

[69] V. Tarokh, H. Jafarkhani, and A. Calderbank, "Space-Time Block Coding for Wireless Communications:Performance Results," in IEEE Journal on Selected Areas in Communications, pp. 451-460, March 1999.

[70] A. Hottinen, O. Tirkkonen, and R. Wichman, Multi-Antenna Tansceiver Techniques for $3 G$ and Beyond. John Wiley and Sons Ltd, 2003.

[71] M. Frodigh, S. Parkvall, C. Roobol, P. Johansson, and P. Larsson, "Future-generation wireless networks," in IEEE Personal Communications, pp. 10-17, October 2001.

[72] M. Steer, "Beyond 3G," in IEEE Microwave Magazine, pp. 76-82, February 2007.

[73] J. Duplicy, B. Badic, R. Balraj, R. Ghaffar, P. Horvath, F. Kaltenberger, R. Knopp, I. Kovacs, H. Nguyen, D. Tandur, and G. Vivier, "MU-MIMO in LTE Systems," in EURASIP Journal on Wireless Communications and Networking, pp. 1-13, Mar 2011.

[74] S. Glisic, Advanced Wireless Communications: 4G Technologies. John Wiley and Sons Ltd, 2004. 
[75] J. Lee, J. Han, and J. Zhang, "MIMO Technologies in 3GPP LTE and LTE-Advanced," in EURASIP Journal on Wireless Communications and Networking, pp. 1-10, July 2009.

[76] R. Mesleh, H. Elgala, and H. Haas, "On the Performance of Different OFDM Based Optical Wireless Communication Systems," in Journal of optical communication network, pp. 620-628, August 2011.

[77] J. Armstrong, B. Schmidt, and D. K. H. S. A. Lowery, "Performance of Asymmetrically Clipped Optical armsOFDM in AWGN for an Intensity Modulated Direct Detection System," in IEEE Global Telecommunications Conference GLOBECOM, pp. 1-5, November 2006.

[78] S. Dimitrov, S. Sinanovic, and H. Haas, "A comparison of OFDM-based modulation schemes for OWC with clipping distortion," in IEEE GLOBECOM Workshops, 2011, pp. 787-791, December 2011.

[79] R. Mesleh, H. Elgala, and H. Haas, "Performance analysis of indoor OFDM optical wireless communication systems," in IEEE Wireless Communications and Networking Conference (WCNC), 2012, pp. 1005-1010, April 2012.

[80] H. Elgala, R. Mesleh, and H. Haas, "Practical considerations for indoor wireless optical system implementation using OFDM," in IEEE International Conference on Telecommunications (ConTEL), 2009, pp. 25-29, June 2009.

[81] S. Dimitrov, S. Sinanovic, and H. Haas, "Clipping Noise in OFDM-Based Optical Wireless Communication Systems," in IEEE Transactions on Communications, pp. 10721081, April 2012.

[82] D. Tsonev, S. Sinanovic, and H. Haas, "Novel Unipolar Orthogonal Frequency Division Multiplexing (U-OFDM) for Optical Wireless," in IEEE Vehicular Technology Conference (VTC Spring), 2012, pp. 1-5, May 2012.

[83] S. Dimitrov, S. Sinanovic, and H. Haas, "Pulse Shaping in Unipolar OFDM-based Modulation Schemes," in IEEE Globecom Workshops, 2012 IEEE, pp. 1208-1212, December 2012.

[84] S. Dimitrov and H. Haas, "On the Clipping Noise in an ACO-OFDM Optical Wireless Communication System," in IEEE Global Telecommunications Conference (GLOBECOM), 2010, pp. 1-5, December 2010.

[85] S. Dimitrov, S. Sinanovic, and H. Haas, "Double-Sided Signal Clipping in ACO-OFDM Wireless Communication Systems," in IEEE International Conference on Communications (ICC), 2011, pp. 1-5, June 2011.

[86] F. Barrami, Y. Guennec, E. Novakov, and P. Busson, "An Optical Power Efficient Asymmetrically Companded DCO-OFDM for IM/DD Systems," in Wireless and Optical Communication Conference (WOCC), 2014, pp. 1-6, May 2014.

[87] S. Dimitrov and H. Haas, "Information Rate of OFDM-Based Optical Wireless Communication Systems With Nonlinear Distortion," in Jounal of Lightwave Technology, pp. 918-929, January 2013. 
[88] R. Mesleh, H. Elgala, and H. Haas, "An overview of indoor OFDM/DMT optical wireless communication systems," in International Symposium on Communication Systems Networks and Digital Signal Processing (CSNDSP), 2010, pp. 566-570, July 2010.

[89] L. Zeng, D. O’Brien, H. Le-Minh, L. Kyungwoo, D. Jung, and O. Yunje, “Improvement of Data rate by using Equalization in an indoor Visible Light Communication system," in IEEE conference in Circuits and Systems for Comarmsmunications, vol. 4, pp. 678-682, May 2008.

[90] R. Mesleh, H. Algala, and H. Haas, "Optical Spatial Modulation," in Journal of Optical Communication and Networking, IEEE/OSA, pp. 234-244, March 2011.

[91] W. Popoola, Z. Ghassemlooy, E. Leitgeb, and V. Ahmadi, "Terrestrial Free-Space Optical Links with Temporal Diversity," in International Symposium on Communication Systems Networks and Digital Signal Processing (CSNDSP), 2010, pp. 598-603, July 2010.

[92] J. Tang and Y. Chang, "Design of an omnidirectional multibeam Transmitter for Highspeed Indoor Wireless Communications," in EURASP Journal on Wireless Communications and networking, vol. 2010, pp. 65-75, April 2010.

[93] Kamelian Hamilton Int. Technology Park, "Semiconductor Optical Amplifiers (SOAs) as Pre-Amplifiers." http://www.kamelian.com/techarticles/ App_Note_No_0 002 .pdf. Applications Note No. 0002, Accessed April 29, 2013.

[94] D. O'Brien, "Multi-input multi-output (MIMO) indoor optical wireless communications," in Conference Record of the Forty-Third Asilomar Conference on Signals, Systems and Computers, pp. 1636-1639, November 2009.

[95] Z. Cheng, N. Seramovski, and H. Haas, "Angle diversity for an Indoor cellular Visible Light Communication System," in IEEE Vehicular Technology Conference Spiring (VTC) 2014, May 2014.

[96] L. Ping, L. Xiao, K. Yeo, T. Wong, and Y. Guan, "MIMO-diversity switching techniques for digital transmission in visible light communication," in IEEE Symposium on Computers and Communications (ISCC), 2013, pp. 576-582, July 2013.

[97] N. Saha, R. Kumar, and M. Jang, "Receiver performance improvement utilizing diversity in MIMO VLC," in International Conference on ICT Convergence (ICTC), 2013, pp. 546-549, October 2013.

[98] A. Burton, H. Minh, Z. Ghassemlooy, E. Bentley, and C. Botella, "Experimental Demonstration of 50-Mb/s Visible Light Communications Using 4 x 4 MIMO," in IEEE Photonics Technology Letters, pp. 945-948, March 2014.

[99] N. Saha and Y. Jang, "Analysis of imaging diversity for MIMO visible light communication," in International Conference on Ubiquitous and Future Networks (ICUFN), 2014, pp. 29-34, July 2014.

[100] N. Tran, D. Luong, T. Thang, and A. Pham, "Performance analysis of indoor MIMO visible light communication systems," in IEEE International Conference on Communications and Electronics (ICCE), 2014, pp. 60-64, August 2014. 
[101] T. Chen, L. Liu, B. Tu, Z. Zheng, and W. Hu, "High-Spatial-Diversity Imaging Receiver Using Fisheye Lens for Indoor MIMO VLCs," in IEEE Photonics Technology Letters, pp. 2260-2263, September 2014.

[102] S. Grant, J. Cheng, L. Krasny, K. Molnar, and Y. Wang, "Per-antenna-rate-control (PARC) in frequency selective fading with SIC-GRAKE receiver," in IEEE Vehicular Technology Conference VTC (2004), pp. 1458-1462, September 2004.

[103] R. Mesleh, H. Haas, C. A, and Y. Sangboh, "Spatial Modulation-A New Low Complexity Spectral Efficiency Enhancing Technique," in First International Conference on Communications and Networking in China, 2006. ChinaCom '06, pp. 1-5, October 2006.

[104] R. Mesleh, H. Haas, S. Sinanovic, and A. C. Y. Sangboh, "Spatial Modulation," in IEEE Vehicular Technology Conference VTC (2008), pp. 2228-2241, July 2008.

[105] R. Mesleh, M. D. Renzo, H. Haas, and M. Grant, "Trellis Coded Spatial Modulation," in IEEE Transactions on Wireless Communications, vol. 9, pp. 2349-2361, July 2010.

[106] M. D. Renzo, H. Haas, S. Sinanovic, and A. C. Y. Sangboh, "Spatial Modulation for Generalized MIMO: Challenges, Opportunities, and Implementation," in Proceedings of the IEEE, vol. 4, pp. 56-103, December 2013.

[107] W. Popoola, "Merits and Limitations of Sarmspatial Modulation for Optical Wireless Communications," in International Workshop on Optical Wireless Communications (IWOW), 2013, pp. 152-156, October 2013.

[108] A. Molisch and M. Win, "MIMO systems with antenna selection," in IEEE Microwave Magazine, vol. 5, pp. 46-56, August 2004.

[109] S. Sanayei and A. Nosratinia, "Antenna selection in MIMO systems," in IEEE Microwave Magazine, vol. 42, pp. 68-73, October 2004.

[110] R. Rajashekar, K. Hari, and L. Hanzo, "Antenna selection in spatial modulation systems," in IEEE Communications Letters, pp. 521-524, January 2013.

[111] D. De-qian and K. Xi-zheng, "A New Indoor VLC Channel Model Based on Reflection," in Springer optoelectronics letters, pp. 295-298, July 2010.

[112] K. Dambul, D. O'Brien, and G. Faulkner, "Indoor Optical Wireless MIMO System With an Imaging Receiver," in IEEE Photonics Technology Letters, pp. 97-99, November 2010 .

[113] T. Fath, H. Haas, M. Renzo, and R. Mesleh, "Spatial Modulation Applied to Optical Wireless Communications in Indoor LOS Environments," in IEEE Global Telecommunications Conference (GLOBECOM 2011), pp. 1-5, December 2011.

[114] T. Fath, J. Klaue, and H. Haas, "Coded spatial modulation applied to optical wireless communications in indoor environments," in IEEE Wireless Communications and Networking Conference (WCNC) 2012, pp. 1000-1004, April 2012.

[115] T. Wang, Y. Sekercioglu, and J. Armstrong, "Hemispherical lens based imaging receiver for MIMO optical wireless communications," in IEEE Globecom Workshops (GC Wkshps) 2012, pp. 1239-1243, December 2012. 
[116] Y. S. T. Wang and J. Armstrong, "Analysis of an Optical Wireless Receiver Using a Hemispherical Lens With Application in MIMO Visible Light Communications," in Journal of Lightwave Technology, pp. 1744-1754, April 2013.

[117] D. Tsonev, S. Sinanovic, and H. Haas, "Practical MIMO Capacity for Indoor Optical Wireless Communication with White LEDs," in IEEE Vehicular Technology Conference (VTC Spring), 2013, pp. 1-5, June 2013.

[118] T. Wang and J. Armstrong, "Performance of Indoor MIMO Optical Wireless System Using Linear Receiver with Prism Array," in Australian Communications Theory Workshop (AusCTW), 2014, pp. 51-56, February 2014.

[119] T. Wang, R. Green, and J. Armstrong, "Prism array-based receiver with application in MIMO indoor optical wireless communications," in International Conference on Transparent Optical Networks (ICTON), 2014, pp. 1-4, July 2014.

[120] Y. Wang and N. Chi, "Demonstration of High-Speed 2 x 2 Non-Imaging MIMO Nyquist Single Carrier Visible Light Communication With Frequency Domain Equalization," in Journal of Lightwave Technology, pp. 2087-2093, April 2014. 